University of Louisville

ThinkIR: The University of Louisville's Institutional Repository

Electronic Theses and Dissertations

$12-2006$

\title{
A robotics testbed : the design \& implementation with applications.
}

Travis Alan Riggs

University of Louisville

Follow this and additional works at: https://ir.library.louisville.edu/etd

\section{Recommended Citation}

Riggs, Travis Alan, "A robotics testbed : the design \& implementation with applications." (2006). Electronic Theses and Dissertations. Paper 1207.

https://doi.org/10.18297/etd/1207

This Master's Thesis is brought to you for free and open access by ThinkIR: The University of Louisville's Institutional Repository. It has been accepted for inclusion in Electronic Theses and Dissertations by an authorized administrator of ThinkIR: The University of Louisville's Institutional Repository. This title appears here courtesy of the author, who has retained all other copyrights. For more information, please contact thinkir@louisville.edu. 


\title{
A ROBOTICS TESTBED: THE DESIGN \& IMPLEMENTATION WITH APPLICATIONS
}

\author{
By \\ Travis Alan Riggs \\ Bachelor of Science in Electrical Engineering, 2005 \\ Speed School of Engineering \\ University of Louisville
}

\begin{abstract}
A Thesis
Submitted to the Faculty of the

University of Louisville

Speed School of Engineering
\end{abstract}

as Partial Fulfillment of the Requirements

for the Professional Degree of

\section{MASTER OF ENGINEERING}

Department of Electrical Engineering

December 2006 



\title{
A ROBOTICS TESTBED: THE DESIGN \& IMPLEMENTATION WITH APPLICATIONS
}

\author{
Submitted by
}

Travis Alan Riggs

A Thesis Approved on

(Date)

by the Following Reading and Examination Committee:

\begin{tabular}{c}
\hline Tamer Inanc, Thesis Director \\
\hline Joseph D. Cole \\
\hline Andrea Kelecy
\end{tabular}




\begin{abstract}
In order to study the movements of autonomous mobile robots, a tool is needed to quantify those movements. A testbed is an apparatus that provides a designated space for multiple mobile robots while tracking their position in real-time. That tracking information can also be communicated to the robots themselves to serve as closed-loop feedback. With this tool, many techniques can be developed and validated through various control experiments. A design and implementation of a testbed is presented here. The testbed is analyzed for its performance and several applications are presented to demonstrate its usefulness.
\end{abstract}




\section{ACKNOWLEDGMENTS}

The author gives all praise and honor to God for the ability to complete this project.

The author would like to thank the thesis advisor, Tamer Inanc, for his direction. He would also like to thank his lovely wife, Jaime, for her continued support through this undertaking. 


\section{TABLE OF CONTENTS}

\section{CHAPTER}

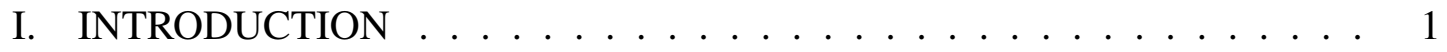

II. DESIGN OF 2D TESTBED . . . . . . . . . . . . . . . . . . . . . 3

A. Design Goals ...................... 3

B. Physical Testbed Design ................. 4

C. Software Algorithms .................. 5

1. Single Robot . . . . . . . . . . . . . . . 6

2. Multiple Robots ................. 9

D. Wireless Communication . . . . . . . . . . . . . . . 11

III. IMPLEMENTATION OF 2D TESTBED . . . . . . . . . . . . . 13

A. Hardware Implementation . . . . . . . . . . . . . . . . 13

1. Testbed Floor Construction . . . . . . . . . . . . 13

2. Camera Location . . . . . . . . . . . . . . 15

3. Camera Mount Construction . . . . . . . . . . . . . . . 19

B. Software Implementation . . . . . . . . . . . . . . . 21

1. Efficient Enhancements . . . . . . . . . . . . . 22

2. Challenges ................... 29

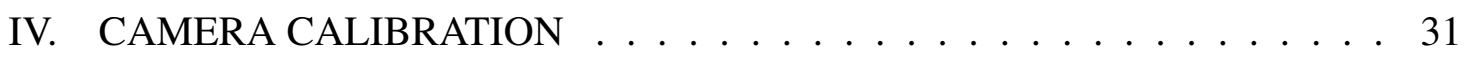

A. Field of View . . . . . . . . . . . . . . . 32

B. Multi-Camera Color Consistency . . . . . . . . . . . . 34

1. A Word About Color Spaces . . . . . . . . . . . . . . . . . . 34

C. Color Threshold Calibration . . . . . . . . . . . . . . . . 37

D. Position Calibration .................... 45 
V. PERFORMANCE OF 2D TESTBED . . . . . . . . . . . . . 47
A. Accuracy . . . . . . . . . . . . . . . 47
B. Speed ...................... 48
C. Other Goals . . . . . . . . . . . . . . . . . . . . . 49
D. Robustness to Noise . . . . . . . . . . . . . 50

VI. MULTIPLE MOBILE ROBOTICS PLATFORM . . . . . . . . . . . . 52

A. Hardware . . . . . . . . . . . . . . . . . 52

B. Software ...................... 53

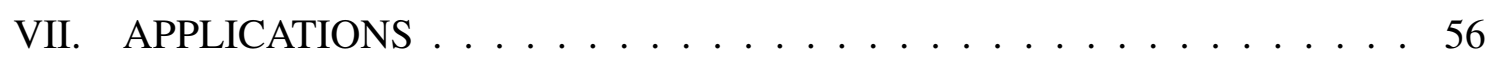

A. Smooth Path Control . . . . . . . . . . . . . . 56

B. Circular Path Control . . . . . . . . . . . . . . 60

1. Simulation ................... 61

2. Single Robot: Odometry and Camera System Feedback _. . . 63

C. Robust Identification of a Single Robot . . . . . . . . . . 66

1. Modeling with Odometry Feedback . . . . . . . . . . . 67

D. Leader-Follow Experiments . . . . . . . . . . . . . 70

1. Simulation .................. 71

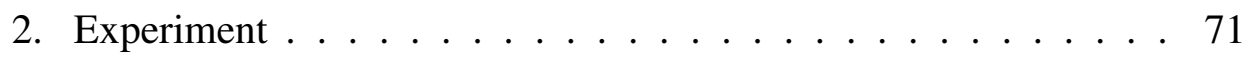

VIII. EXTENSION TO 3D: A TESTBED DESIGN FOR SMALL-SCALE AERIAL VEHICLES . . . . . . . . . . . . . . . . . . 77

A. Need for a 3D Testbed . . . . . . . . . . . . . . . 77

B. Analysis \& Design . . . . . . . . . . . . . . . . . . 77

1. From $2 \mathrm{D}$ to $3 \mathrm{D} \ldots \ldots \ldots \ldots \ldots \ldots \ldots \ldots$

2. Calculation of the Pose . . . . . . . . . . . 80

IX. CONCLUSIONS \& FUTURE WORKS . . . . . . . . . . . . . . 84

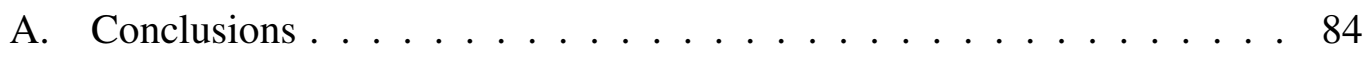

B. Future Works . . . . . . . . . . . . . 85 


\section{APPENDIX}

I. PROGRAM 1: Single Robot Tracking . . . . . . . . . . . . . . . . . 87

II. PROGRAM 2: Multi-Robot Tracking . . . . . . . . . . . . . . . . 101

III. Color Threshold Calibration Data . . . . . . . . . . . . . . . 123

IV. Color Threshold Calibration Sampling Program . . . . . . . . . . . . . 126

V. Color Threshold Calibration Program . . . . . . . . . . . . . . . 132

VI. Kernel Modification Files . . . . . . . . . . . . . . . . . . 135

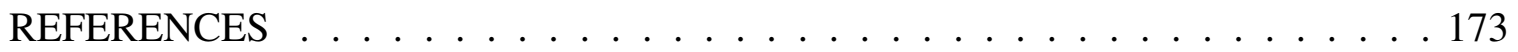

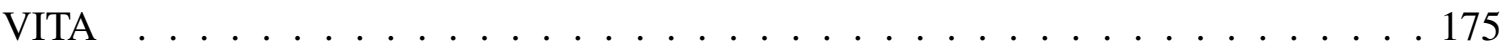




\section{LIST OF FIGURES}

FIGURE 1. A common setup for a mobile robotics testbed, using overhead cameras to track movements of robots on the floor below. . . . . . . . . 5

FIGURE 2. Specialized color "hat" pattern worn on top of a single robot, to be identified by the overhead camera system for tracking. The red block is worn on the front of the robot with the blue on the back. $\ldots \ldots$. . 6

FIGURE 3. Illustration of the reference frames for the robot. The testbed measures in the global reference frame $\left(X_{G}, Y_{G}, \theta\right)$ as defined in the figure. The robot's local reference frame $\left(X_{R}, Y_{R}\right)$ is shown superimposed on

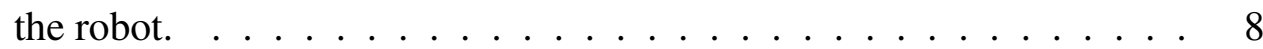

FIGURE 4. Specialized color "hat" template pattern for multiple robots. The red circle in the center is used for the $x \& y$ calculation. The black/white semi-circles on the outside are for the orientation measurement. Four quarter circles surround the red center, shown here in green, are each filled with one of three colors (Green, Blue and White) to encode the robot number into the pattern making each hat unique. . . . . . . 10

FIGURE 5. CAD drawings of two possible configurations for the laboratory space. The first layout was chosen because it more efficiently utilized the space. 14

FIGURE 6. Image of actual testbed floor. The short fence is in the bottom of the image. The walls enclose the other sides. . . . . . . . . . . . 15

FIGURE 7. Three dimensional model used to simulate placement of the cameras in the room. The large, black bar in the model represents the lights that hangs 12" below the ceiling. Measurements calculated from this model were used to construct the physical testbed. . . . . . . . . . 16 
FIGURE 8. A gray-scale image of a section of the testbed with two robots. The cameras were carefully tuned so that they would combine well in the center of the testbed. The robot on the right is in the center and there is a nice image of the pattern. Although the image is nice here, that is not the case for another area of the testbed. The robot on the left shows the schism effect of lens distortion. . . . . . . . . . . . . 17

FIGURE 9. This is an image taken through a lens with barrel distortion. The grid lines should all be orthogonal. There is a difference in magnification levels between the center of the lens and the outside of the lens. . . . 18

FIGURE 10. Testbed model shown at camera height used for actual testbed. With respect to the origin shown in the model, the camera lenses are located at the following positions: (36.8", 50.0", 112.0"), (36.8”, 123.5", 112.0”), (97.2”, 50.0”, 112.0”), (97.2”, 123.5”, 112.0”) . . . . . . 19

FIGURE 11. Mounting beams used to support cameras. Slotted holes allow them to be mounted anywhere along the beam for flexibility during calibration. 20

FIGURE 12. Mounting platforms that hold cameras. Slotted regions allow the cameras to slide anywhere along the platform. . . . . . . . . . 21

FIGURE 13. Close-up of mounting brackets or discs used to attach L-arm to mounting platform. The discs sandwich the platform, providing a solid base while still allowing the cameras to rotate, if needed. . . . . . . . . 22

FIGURE 14. View of all the camera mounting hardware for the testbed. . . . . . 23

FIGURE 15. Example of a thresholding lookup table for the color orange in the RGB color space. 1's represent an acceptable match level. If the lookup table returns three 1's, the pixel will be classified as orange. . . 24 
FIGURE 16. Histograms of the colors blue and black in the YCbCr colorspace. Notice the overlap in the $\mathrm{Y}$ and $\mathrm{Cr}$ channels. There is also little separation in the $\mathrm{Cb}$ channel, making these two colors difficult to distinguish, especially in changing lighting conditions. . . . . . . . 25

FIGURE 17. Search method and center calculation used in [7]. The sub-sampled search method was used for the local testbed, but a different algorithm was chosen for pose calculations. White squares indicate not finding the blue circle. Red square indicates a "hit" from the search for blue. Green pixels are measuring chords of the circle. Purple squares are the center of each chord. White lines show perpendicular bisectors, which intersect at the center of the blue circle, a.k.a. the center of the

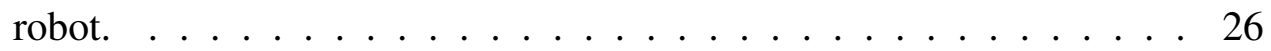

FIGURE 18. Visual representation of the sub-sampled search algorithm. White represents pixels that were not examined. The black dots show what pixels were thresholded. The black box shows where the refined area search took place. This is the search for the single robot. The green dots represent the centers of the red block and the blue block. Notice that the algorithm stops searching once the single robot is found. . . . 28

FIGURE 19. 3D view of cone angle measurements taken from video camera at $1 \mathrm{x}$ zoom. Red points represent measurements taken in the vertical plane. Blue points are measured in the horizontal plane. Green lines show least squares fit. Rectangle shows field of view at a distance of 110”. . 33

FIGURE 20. 3D view of cone angle measurements taken from video camera at 2x Zoom on the left, 5x Zoom on the right. Red points represent measurements taken in the vertical plane. Blue points are measured in the horizontal plane. Green lines show least squares fit. Rectangle shows field of view at a distance of 110" . . . . . . . . . . 33 
FIGURE 21. Picture of Cardinal quarterback Brian Brohm throwing a pass against the Syracuse Orangemen. Used here to illustrate the $\mathrm{YCbCr}$ color space. Left, is the original image. The images to the right of the original are the $\mathrm{Y}, \mathrm{Cb}$ and $\mathrm{Cr}$ represented as gray-scale images. . . . . 34

FIGURE 22. Frame captured from a video camera of the image used to calibrate all cameras. Print consists of a block of pure red, blue, green, black

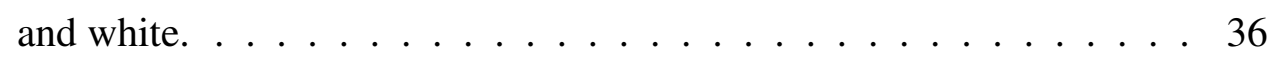

FIGURE 23. Histogram of calibration print, taken from Camera 1, with desirable hardware settings. This provided the most separation between colors in the $\mathrm{YCbCr}$ color space. . . . . . . . . . . . . . . 37

FIGURE 24. Histogram of calibration print, taken from Cameras 0, 2 and 3, to match the benchmark of Camera 1 . The histograms match very well with that shown in Fig. $23 \ldots . . \ldots 38$

FIGURE 25. Histograms of the color red in the RGB and YCbCr spaces, taken from Camera 0, in order to determine proper thresholding range for

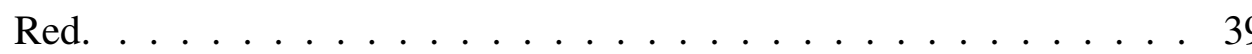

FIGURE 26. From the Left: YCbCr Histograms of Red, Green, Blue, Black and White, taken from samples of all cameras, in order to determine proper thresholding range for the colors. The bounding boxes shown on the histograms indicate good thresholding ranges. . . . . . . . . . . . 41

FIGURE 27. Threshold ranges in the YCbCr space for the colors Red, Green, Blue, Black and White. White is shown as Yellow for visibility. . . . . . . . 42

FIGURE 28. Three dimensional plots of the colors Red, Green, Blue, Black and White in the YCbCr color space. White is shown as Yellow for visibility. 42 
FIGURE 29. Thresholded version of Fig. 8. This was thresholded in the YCbCr color space and turned out very well. The purple pixels represent ones that were not classified as any one of the primary colors: Red, Green, Blue, Black or White. . . . . . . . . . . . . . . . . . 43

FIGURE 30. Example of discretization error caused by sampling a continuous image. The left shows the continuous image of a circle with a sampling grid over the image. The right shows the low-resolution sampling of the continuous image. . . . . . . . . . . . . . . . 44

FIGURE 31. Scatter Plot and Histogram of 10,000 measurements for a stationary robot using the single robot algorithm and hat pattern. Notice the scale of the axes is $10^{-3} \ldots \ldots \ldots \ldots$

FIGURE 32. Scatter Plot and Histogram of 10,000 measurements for a stationary robot using the multi-robot algorithm and hat pattern. Notice the scale of the axes is $10^{-3} \ldots \ldots \ldots \ldots$

FIGURE 33. Scatter Plot and Histogram of 10,000 measurements for a stationary robot using the multi-robot algorithm and hat pattern. To add noise to the system, some of the overhead lighting was shut off. Notice the scale of the axes is still $10^{-3}$, though the variance has increased. . . . 50

FIGURE 34. Picture of 3 Evolution Robotics ER1's. The robot bodies can be reconfigured to build a variety of shapes. The standard configuration is on the left. The robot to the right is the hitch and trailer style. All of these robots are shown wearing their "hats", for tracking by the camera system. ..................... 5

FIGURE 35. Diagram of the robot's frames of interest. The control variables $\rho, \alpha$, and $\beta$ are illustrated. The goal here is shown in the upper-right, with the orientation aligned with the global axes. . . . . . . . . . 57 
FIGURE 36. Stage simulation of the smooth trajectory controller. The robot was placed at different poses on a $6 \mathrm{~m}$ radius circle. The starting orientation angle was always $0^{\circ}$. The robot found a smooth path to the origin each time. . . . . . . . . . . . . . . . 59

FIGURE 37. Stage simulation and actual experiment of the smooth trajectory controller. The command was to move from the $\left(0,0,0^{\circ}\right)$ to $\left(0,2,0^{\circ}\right)$. The robot moved along a smooth path in the simulator and the hardware. The hardware shows a small steady-state error though. . . . . . . . 60

FIGURE 38. Simulation of circular path controller, tracking the radius at 3 meters, with a forward speed of $0.2 \mathrm{~m} / \mathrm{s}$. The starting point was $\left(3.0,0,90^{\circ}\right)$. As time progressed, the oscillations grew until the algorithm failed. . . 62

FIGURE 39. Simulation of circular path controller, tracking the radius of 3 meters and the tangent angle of the circle, with a forward speed of 0.2 $\mathrm{m} / \mathrm{s}$. Even when the starting location was the origin, this controller performed very well. . . . . . . . . . . . . 63

FIGURE 40. Experiment of circular path controller in testbed. Tracking is done for a 0.5 radius and forward speed of $0.2 \mathrm{~m} / \mathrm{s}$. Plot shows the experiment with odometry feedback in blue, and camera system feedback in red. There is a steady-state error present in this controller. . . . . . . . . 64

FIGURE 41. Experiment of circular path controller in testbed. Tracking is done for a 0.5 radius and forward speed of $0.2 \mathrm{~m} / \mathrm{s}$. Plot shows the experiment with odometry feedback in blue, and camera system feedback in red in three dimensions to view the change in $\theta$. The discontinuity is expected as $\theta$ moves from $180^{\circ}$ to $-180^{\circ} \ldots \ldots . . . . . . . .665$

FIGURE 42. A summary of the Robust Identification Framework. . . . . . . . . . . 66 
FIGURE 43. Result of robust identification using a 2 meter step input. The identification was done using the internal odometers as feedback. The full order and reduced order models are shown in black and red, respectively. The actual output is shown in blue. The reduced order model was reduced from $25^{\text {th }}$ order model to a $2^{\text {nd }}$ order model. . . . . . . 67

FIGURE 44. Robust model comparison in the frequency domain. The frequency data is shown for the actual data, the full order model and the reduced model. The model matches the data well in the time and frequency domains. ...................... 68

FIGURE 45. Result of robust identification using a 2 meter step input. The modeling was done using the camera system as feedback. The full order and reduced order models are shown in black and red, respectively. The actual output is shown in blue. The reduced order model was reduced from $25^{\text {th }}$ order model to a $2^{\text {nd }}$ order model. . . . . . . . . . . .

FIGURE 46. Robust model comparison in the frequency domain. The frequency data is shown for the actual data, the full order model and the reduced model. The model matches the data well in the time and frequency

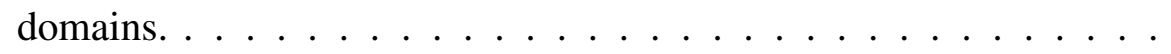

FIGURE 47. Simulation of a leader-follow algorithm, with the leader given the objective to track a circle. The leader is shown in red and the follower in green. The initial position of the robots are near the origin as shown, with the follower 1 meter behind the leader. . . . . . . . . . 72

FIGURE 48. Simulation of a leader-follow algorithm, with the leader given two separate movement commands. The first command was for the leader to go to the pose $\left(2,3.5,0^{\circ}\right)$. Once that was reached, a second command pose was given as $\left(5,1.5-90^{\circ}\right)$. The leader is shown in red and the follower in green. . . . . . . . . . . . . 73 
FIGURE 49. Leader-Follow experiment with ER1 robots. The robot was given a circle to track. The initial location of the follower was approximately $0.5 \mathrm{~m}$ behind the leader. . . . . . . . . . . . . . . . 74

FIGURE 50. Diagram of result of noise in the system. When the leader makes a small change in $\theta$, the goal for the follower makes a larger change. This is the reason that there is more variance in the follower's path. . . 75

FIGURE 51. Leader-Follow-Follow experiment with ER1 robots. The leader robot, Adam, shown in red, was given a circle to track. The first follower, Abel, shown in green tried to track Adam. The second follower, Eve, shown in green, tries to follow Abel. The last robot is the tractortrailer robot design seen earlier. . . . . . . . . . . . . . 76

FIGURE 52. Spherical coordinate system of the camera, with the camera at the origin and an aerial vehicle shown in the first octant. A light ray extends from the center of the vehicle to the camera lens. This light ray can be described by its spherical coordinate angles $\theta$ and $\phi$. . . . 78

FIGURE 53. Camera coverage of four cameras suspended from the ceiling. Each camera is located at the point of each "pyramid". The pyramids overlap creating this shape which shows the viewable space of the cameras. 79

FIGURE 54. Camera coverage of four cameras mounted on a wall. These would be used to capture the $z$ value, or altitude, of a robot. Each camera is located at the tip of the "pyramid" shape which shows the camera's viewable space. Here the viewable spaces overlap slightly. . . . . . . 79 
FIGURE 55. This figure shows two cameras mounted very close to each other in an upper corner of the proposed testbed. They are angled downward slightly at the same $\phi$ angle but have different horizontal (or $\theta$ ) angles. The viewable spaces overlap slightly. For clarity, the left camera's viewable space pyramid is a different color than the right camera's pyramid. . . . . . . . . . . . . . . 80

FIGURE 56. The same as Fig. 55, except two more cameras are added to the adjacent corner. The pyramids are shown as semi-transparent to more easily see the total coverage. The entire upper section of the testbed is covered by the cameras. . . . . . . . . . . . . 81

FIGURE 57. An overhead view of two cameras mounted in adjacent corners a distance $d$ from each other, at arbitrary angles $\rho_{1}$ and $\rho_{2}$. The ellipse shown is an aerial vehicle. The cameras are used to measure $\theta_{1}$ and $\theta_{2}$ and triangulate the vehicle's $x$ and $y$ coordinates. . . . . . . 81

FIGURE 58. The cameras from Fig. 57 seen from the side. They are both at the same position and downward orientation $\delta$. The ellipse is the aerial vehicle. The cameras both measure the same angle $\phi$, which is then used with the recently found value of $x$ to calculate $z$, the altitude of the vehicle. ..................... . . . 82

FIGURE 59. Camera 0: From the LEFT: YCbCr Histograms of Red, Green, Blue, Black and White. . . . . . . . . . . . . . 123

FIGURE 60. Camera 1: From the LEFT: YCbCr Histograms of Red, Green, Blue, Black and White. . . . . . . . . . . . . . . . 124

FIGURE 61. Camera 2: From the LEFT: YCbCr Histograms of Red, Green, Blue, Black and White. . . . . . . . . . . . . . . . 124

FIGURE 62. Camera 3: From the LEFT: YCbCr Histograms of Red, Green, Blue, Black and White. . . . . . . . . . . . . . 125 


\section{CHAPTER I INTRODUCTION}

Imagine a robot entering a collapsed building to search for survivors. Picture a machine assisting an elderly woman at the supermarket, or helping her cross the street. Envision a robot inspecting passengers at a border crossing in a war-torn country. Think of autonomous vehicles roaming the surface of nearby planets in search of minerals and resources.

From the dull to the dangerous, robots have the potential to impact our world in a promising way. Machines could perform simple, mundane tasks, freeing people to perform higher level work. Or they could protect lives.

These are just some of the applications of autonomous mobile robotics. Each presents its own challenges and needs, melding a broad range of disciplines from path planning to sensor fusion. To realize these dreams, more work must be done. In order to study some of these areas, a tool must be developed to validate new methods for autonomous robots. For the area of control systems, that tool is a testbed.

Testbeds have been used for some time to study various aspects of robotic movements [1]-[7]. A testbed typically consists of a sizable area for the robot or robots to move around and is completed by some system to measure their movement. Traditionally, this system is vision-based. Video cameras are mounted above and face downward to view the testbed floor. Each robot is tracked from the video feed and the pose information is then communicated to the robots below. In this way, the cameras act like a local global positioning satellite (GPS) system. Equipped with this tool, many new techniques might be developed and validated through different open-loop and closed-loop experiments.

This thesis begins with a discussion of the design of a testbed. The implementation 
of the testbed, including the hardware used and the algorithms developed, are studied in detail. Chapter IV discusses issues encountered with camera calibration, both in hardware and software. The methods used are compared to other modern approaches to camera calibration [8]. The performance of the testbed is then analyzed for speed, accuracy and robustness to noise.

In the latter half of the thesis, Chapter VII discusses applications of this testbed, including the novel robust identification of a single mobile robot. Various controllers are designed and tested in a robot simulator and the recently constructed testbed. A controller is developed to provide a smooth trajectory from any point in the testbed to any other arbitrary point. A second controller is presented that tracks a circular path. The smooth path controller is later extended to coordinated movement between multiple robots. The methods shown here are tested in both a simulated environment as well as the testbed.

Following the applications, an extension to the current testbed is proposed in Chapter VIII to allow three dimensional tracking for aerial vehicles. Until recently, three dimensional testbeds have been built [5] strictly for outdoor use with large aerial vehicles. They employ expensive GPS and inertial sensors to measure the position of the robots. With the availability of small, affordable, aerial vehicles, three dimensional testbeds are being built for indoor use. MIT recently purchased just such a testbed from a company called Vicon [6]. Their new testbed uses high-speed, infrared (IR) cameras to track the position of multiple aerial vehicles. An alternate, low-cost design for a 3D testbed is presented here, which focuses on using small aerial vehicles in an indoor setting. It will also use a vision system to track the pose of multiple robots efficiently. 


\section{CHAPTER II \\ DESIGN OF 2D TESTBED}

\section{A. Design Goals}

Before delving into the detailed design of the testbed, it is important to consider the objectives of this project.

1. Utilize the Lab Space Efficiently

2. Support Multiple Robots

3. Make Accurate Pose Measurements

4. Track Robots in Real-Time

5. Use a Generic Hardware Platform to Track Robots

\section{Communicate Position Data to Mobile Robots}

There are several requirements of this testbed. First, it needs to utilize the space in such a way as to facilitate experiments with multiple mobile robots. Therefore, the testbed could not be long and narrow. A square shape would allow several robots to move in different formations. It would also permit movement in any direction equally. A narrow testbed would constrain the majority of the movement to a single axis.

Second, the testbed needs to support multiple robots. It should be able to measure the position, the $x$ and $y$ coordinate, along with the orientation of each robot on the floor plane. The combination of the $x$ and $y$ coordinates with the orientation, $\theta$, is referred to as the pose, written as $(x, y, \theta)$. 
This testbed is made to emulate a local GPS system. GPS does not function reliably indoors. A GPS receiver detects time signals from four satellites. By measuring the time difference between each signal received, the device can calculate its position. Typical accuracies of GPS systems are reported in the range of meters. This testbed would be required to produce accurate measurements of each robot's pose in the centimeter range. Typical accuracies of other testbeds have been reported at \pm 8 centimeters of the actual position and within 10 or 12 degrees of the actual orientation.

One critical objective of this testbed is to measure the pose information in "realtime". Real-time is a subjective term, but it is typically defined in process control systems as 10 to $60 \mathrm{~Hz}$. The goal of this testbed is to perform at or near 30 pose measurements per second, which is the upper limit of this testbed because of the sensors used: NTSC cameras. The NTSC video standard is 30 frames per second.

Other testbeds have reported tracking frequencies of $30 \mathrm{~Hz}$, but all of them perform the tracking using costly, specialized hardware. Another goal of this testbed is to use a generic software, sensor and computer platform. While saving money, implementing the tracking in software allows the testbed to be modified more easily to meet new require-

ments. Should a special situation arise, a new algorithm can be implemented to satisfy the new objective, all with the existing platform.

In order to make use of the testbed's pose measurements, there must be some method to communicate to the robots. The final goal of the testbed is to effectively communicate pose information to each robot. This testbed will provide a wireless platform to meet this need, but this communication method can be replaced or augmented at a later time.

\section{B. Physical Testbed Design}

This is a vision-based testbed, which means that cameras are used to perform the pose measurements. Video cameras are mounted in the ceiling and face downward toward 


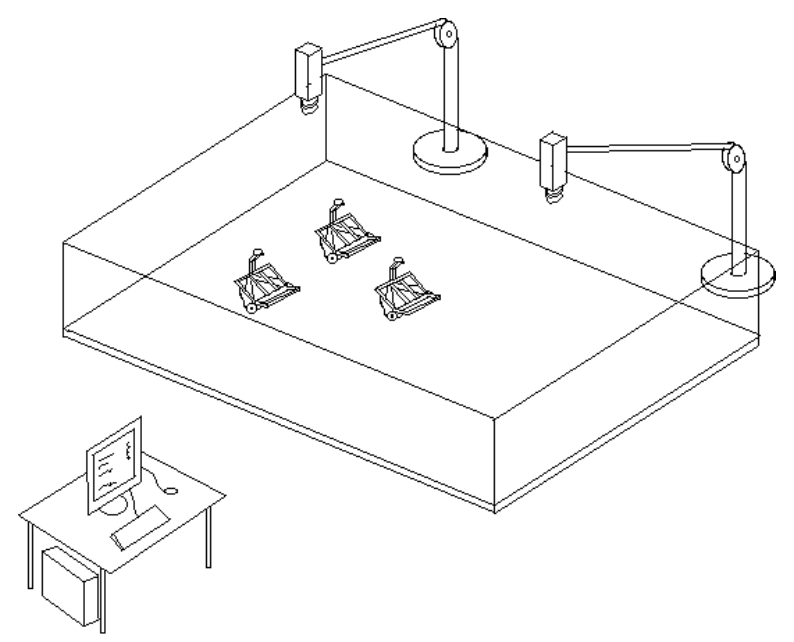

FIGURE 1 - A common setup for a mobile robotics testbed, using overhead cameras to track movements of robots on the floor below.

the floor. Four cameras are used to cover the entire testbed floor. In order to provide a high contrast background for the overhead cameras, the testbed floor is white. The shape of the testbed is a near-square shape to facilitate movement in all directions. It extends as large as possible while still accommodating other needs for the lab space and maximizing the coverage of the cameras. Fig. 1 shows an initial design of the testbed. Also shown in the design sketch is a desktop computer. The computer performs all of the pose measurement calculations from the images captured from the overhead cameras.

\section{Software Algorithms}

The needs of the testbed require the ability to track multiple robots in real-time. However, there are times when only one robot will be studied at time. As a display of the flexibility of the generic platform, two algorithms have been developed to meet two separate needs. The single robot algorithm takes advantage of the simplicity of searching for a single robot, increasing accuracy and the frequency of measurement. This can be used when only one robot is studied, yielding more accurate tracking. The multi-robot algorithm is inherently more complex. The search in this case must not only calculate the position 


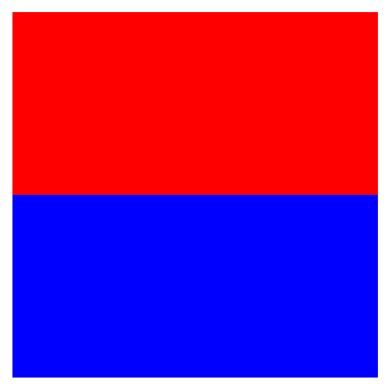

FIGURE 2-Specialized color "hat" pattern worn on top of a single robot, to be identified by the overhead camera system for tracking. The red block is worn on the front of the robot with the blue on the back.

and orientation multiple times, but must also uniquely identify robots. The result is a more involved and therefore, slower measurement.

\section{Single Robot}

The algorithm to search for a single robot is simple. A specialized color pattern is placed on top of the robot, referred to as a "hat". With the robot's body hidden below the hat, the camera is searching for the appropriate pattern. The hat design for the single robot is shown in Fig 2. It consists of a red rectangle abutting a blue rectangle on the long edge. The hat is worn with the red rectangle on the front of the robot, while the blue is on the back. The algorithm is based on two separate center-of-mass measurements of the two colored rectangles on the hat. The center of mass of a two-dimensional object of uniform density - as in an image - is defined in equations (1) and (2):

$$
\begin{aligned}
x_{\text {center }} & =\frac{\iint x d x d y}{\iint d x d y} \\
y_{\text {center }} & =\frac{\iint y d x d y}{\iint d x d y}
\end{aligned}
$$

With digital cameras, the mass distributions are discrete. So, equations (3) and (4) are more appropriate.

$$
\begin{aligned}
& x_{\text {center }}=\frac{\sum \sum x_{k} m_{k}}{\sum \sum m_{k}} \\
& y_{\text {center }}=\frac{\sum \sum y_{k} m_{k}}{\sum \sum m_{k}}
\end{aligned}
$$


In a scan of the camera frames, the image is thresholded for red and blue. The $x$ and $y$ coordinates of each red pixel are summed. The $x$ and $y$ sums are then divided by the mass of the red pixels. This yields the two dimensional average of the red block, or its center. The same process is performed for all blue pixels. Once the centers of the red and blue block is found, it is easy to see that the average of these two centers will yield the center of the robot itself.

$$
(x, y)_{\text {Robot }}=\left(\frac{x_{\text {red }}+x_{\text {blue }}}{2}, \frac{y_{\text {red }}+y_{\text {blue }}}{2}\right)
$$

Using simple geometry, it can be shown that the arctangent of the line drawn from the red center to the blue center will give the orientation angle of the robot as shown in Eq. 6 .

$$
\theta_{\text {Robot }}=\arctan \left(\frac{y_{\text {red }}-y_{\text {blue }}}{x_{\text {red }}-x_{\text {blue }}}\right)
$$

These measurements are taken in the global reference frame of the testbed. The origin of the testbed was chosen as an arbitrary corner. Figure 3 shows this global reference frame, along with the robot's local reference frame. In the global reference frame, the pose is typically represented by $\left(\mathrm{X}_{G}, \mathrm{Y}_{G}, \theta\right)$. The robot's local reference frame, $\left(\mathrm{X}_{R}, \mathrm{Y}_{R}, \theta\right)$, changes with the movement of the robot. A differential drive robot is shown in the figure. The origin in the local reference frame is mid-way between the wheels. The forward direction for the robot is in the $\mathrm{X}_{R}$ direction. Note that $\theta$ is defined the same in the robot's local reference frame as it is in the global reference frame. 


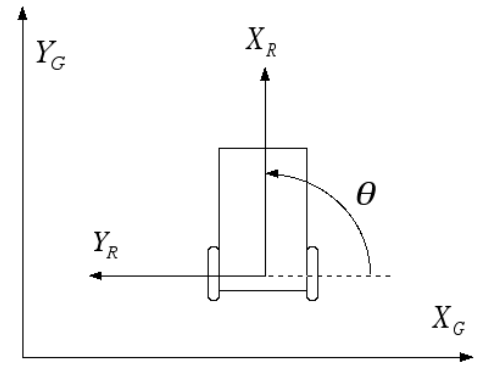

FIGURE 3 -Illustration of the reference frames for the robot. The testbed measures in the global reference frame $\left(X_{G}, Y_{G}, \theta\right)$ as defined in the figure. The robot's local reference frame $\left(X_{R}, Y_{R}\right)$ is shown superimposed on the robot.

This algorithm is summarized in the pseudo-code below.

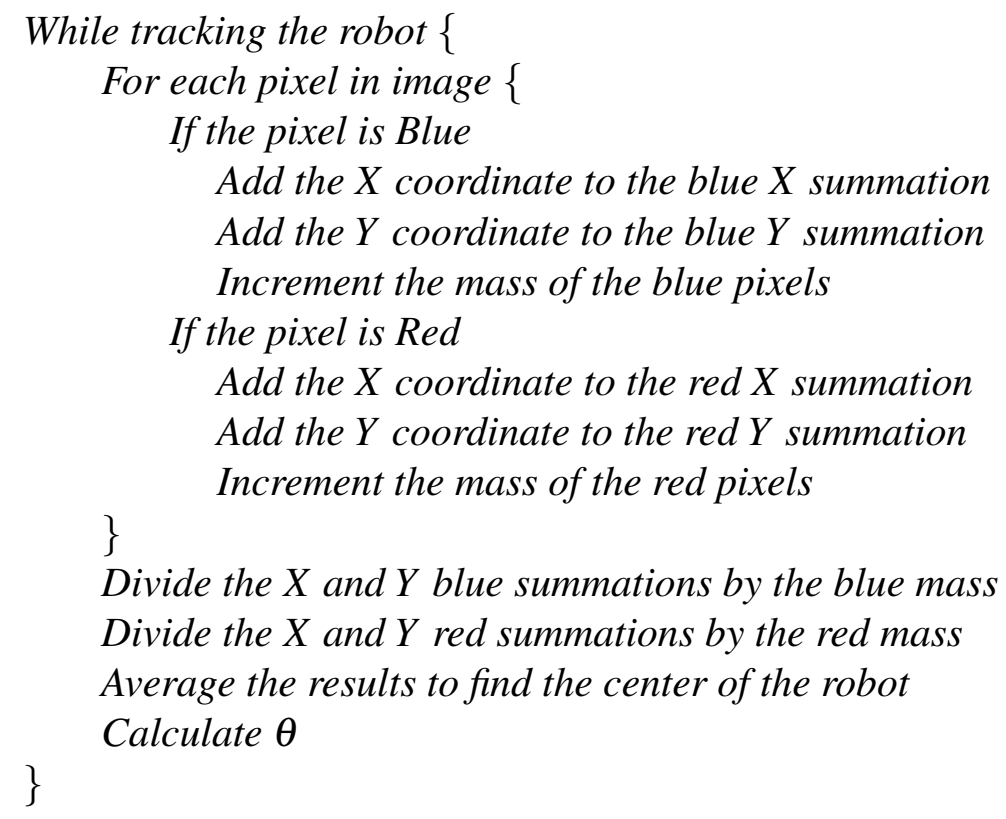

This algorithm has several advantages. First, it is very simple, which provides a faster run-time. It only involves two summations, a couple of division operations and an arc-tangent calculation. It also only requires a single pass over the image. Most computer vision algorithms require multiple loops over every pixel. Even with fast processors, scanning an image with hundreds of thousands of pixels is a time consuming process. A great effort is made to consolidate operation loops. In this case, there is only a single pass over the image, increasing the measurement frequency. 


\section{Multiple Robots}

The algorithm to track multiple robots is more complicated for several reasons. Now, the process of measuring the pose must be repeated for each robot that is found on the surface. Also, once the locations and orientations are found, they must be matched to the correct robot. So, a third operation of uniquely identifying each robot is added to the process.

To accurately measure and identify each robot, a new pattern system is needed. The simple hat could be extended for the use of multiple robots, but only to a point. With the simple algorithm, each robot would require a hat with two unique colors. One would replace the red block while another color would replace the blue. In order to differentiate between the robots, the colors themselves would need to be distinguishable to the cameras

overhead. If the testbed needed to measure eight robots, sixteen different colors would be needed for the hat patterns.

In theory, this is possible. However, in practice, there is often too much noise in an image to classify closely related colors accurately. As a robot moves around the testbed floor, there are slight variations in lighting. Light condition changes have long plagued computer vision research, as many algorithms are susceptible to them. Although there are many statistical, and even spatial, techniques for accurate classification, these methods are far too slow to meet the real-time demand of this system.

This method of using unique colors to identify each robot also reduces the scalability of the testbed. There are a limited number of colors that can be classified uniquely. Depending on the noise level in the system, the number can be as high as 32 . This would allow for only 16 robots. For a large testbed, this number might not be sufficient. For most practical systems, the number of unique colors is closer to eight or nine, only allowing four or five robots on the testbed.

Instead of simply extending the single robot algorithm, an entirely new one was developed. The design template for the hat pattern is shown in Fig. 4. It is only a template, 


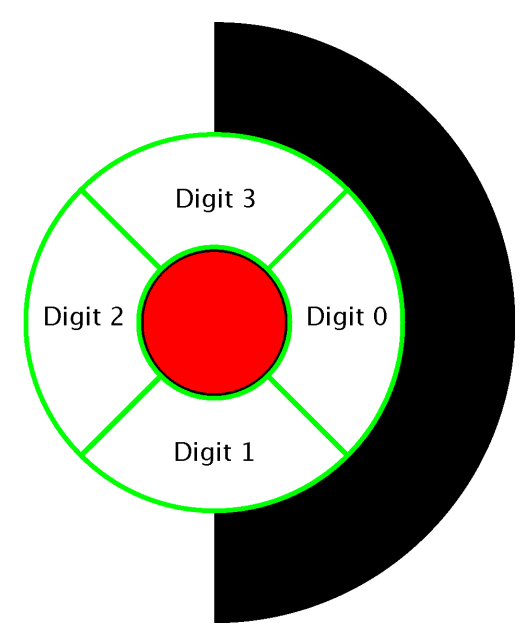

FIGURE 4-Specialized color "hat" template pattern for multiple robots. The red circle in the center is used for the $x \& y$ calculation. The black/white semi-circles on the outside are for the orientation measurement. Four quarter circles surround the red center, shown here in green, are each filled with one of three colors (Green, Blue and White) to encode the robot number into the pattern making each hat unique.

because each hat will have a unique identifier pattern associated with each robot. There are some common features among each hat pattern. The first is the central red circle. Red is a color that has proven to threshold easily and is used here to initially detect a robot.

To save scanning time, the image is sub-sampled to detect a red pixel. In other words, only one pixel will be examined within a neighborhood of several pixels. That pixel is thresholded. If the pixel is some color besides red, it is set aside and a pixel from the next neighborhood is studied. This procedure continues skipping across a grid over the image until a red pixel is located. When this happens, the pixel neighborhood is examined for other red pixels. The center of mass is then calculated for the red pixels discovered in the neighborhood. This gives the center of a robot. At this time it is still not known which robot it might be. Once the center of the red circle is found, the second feature of the hat pattern is used to determine the orientation of the yet-to-be-named robot. The black and white semi-circles surrounding the pattern provide unique points that can be used to find the orientation. Those unique areas are the beginning and end of the semi-circles, or where the black and white shapes meet each other. By moving around the robot's center at a constant radius, the pixels are thresholded until the black-white or white-black edge 
is found. This will give a second point of reference for this robot. This point, along with the center, can be used to calculate the orientation angle, $\theta$, in the same way as the simple algorithm. It is simply the arctangent of the line constructed from these two points.

Now that the location and orientation have been found for the robot, it is time to identify which robot it is. The third feature of this pattern is the inner ring area. Here, the inner ring, outlined in green in Fig. 4, is divided into four equal regions or quarter circles. The robot's number is encoded into this ring using three colors: green, blue and white. Green represents the number two. Blue represents one and white makes zero. With three colors, the encoding scheme is base 3 . The four sections of this ring are filled with the different colors to make up four digits in the scheme. So, the number of unique hat patterns is $3^{4}$ which is 81 . This means the software could support up to 81 different robots. It is safe to say that this number will not be reached, but still leaves plenty of room for expansion.

Once a robot's pose variables have been found, along with its identifier, the search continues across the rest of the image for red pixels. One note here is that care must be taken not to search through previously discovered red pixels. This would lead to calculating the pose variables of an already-discovered robot. To prevent this, once a red pixel is found, its value is changed to an impossible level. This guarantees that it will not threshold as red again.

\section{Wireless Communication}

The initial design of the wireless communication scheme uses a standard protocol known as Universal Datagram Packet (UDP). The options were UDP and Transport Control Protocol (TCP). The UDP standard was selected for its simplicity and flexibility. UDP sends messages as a broadcast. TCP is more complex in that it requires a relationship to be set up using "hand-shaking" with the intended receiver of the message. The protocol sends a message to a receiver and then waits to hear back from the receiver, signifying the message was transmitted correctly. With UDP, there is no hand-shaking, or any request of 
information back from the intended receiver. The message is sent without worrying about the receipt of the message. This involves less overhead for both the vision system and the robots themselves.

For example, a robot may be busy processing data from a sensor mounted on top of it. It may be perfectly still while it makes a decision what to do next. If the robot knows its pose, it would be a waste of time to "listen" for an update. When the robot needs an update of its pose, it can listen for the next broadcast.

Using UDP, the vision system broadcasts the pose information without worrying if the message was received. Consequently, this scheme is less reliable than TCP communication, but speed and simplicity are gained using UDP; both for the vision system and the robot. 


\section{CHAPTER III IMPLEMENTATION OF 2D TESTBED}

This chapter covers the implementation details of the testbed. It begins with the hardware used and closes by discussing software issues.

\section{A. Hardware Implementation}

\section{Testbed Floor Construction}

The lab space given needed to house student researchers as well as the testbed. In order to utilize as much space as possible, the testbed floor stretched wall to wall. Two different configurations are shown in Fig. 5. The first was chosen because it provided more of a square shape while using the space most effectively.

An odd material was used for this floor: dry-erase marker board. The original flooring of the lab was a multi-colored tile designed to be anti-static. This is important for work with small scale electronic devices, such as clean rooms. However, this application depended more on the color of the floor. A single, high-contrast color would allow the overhead camera system to more quickly distinguish between a potential mobile robot and the background. A multi-colored floor would require more image processing. Although the processing would be very simple, it would waste precious time and considerably decrease the frequency of the testbed's measurements. This would jeopardize the real-time goal of the testbed.

The only other requirement of the flooring used was that it would provide enough friction to prevent significant wheel slippage. A dry erase board was tested before construc- 

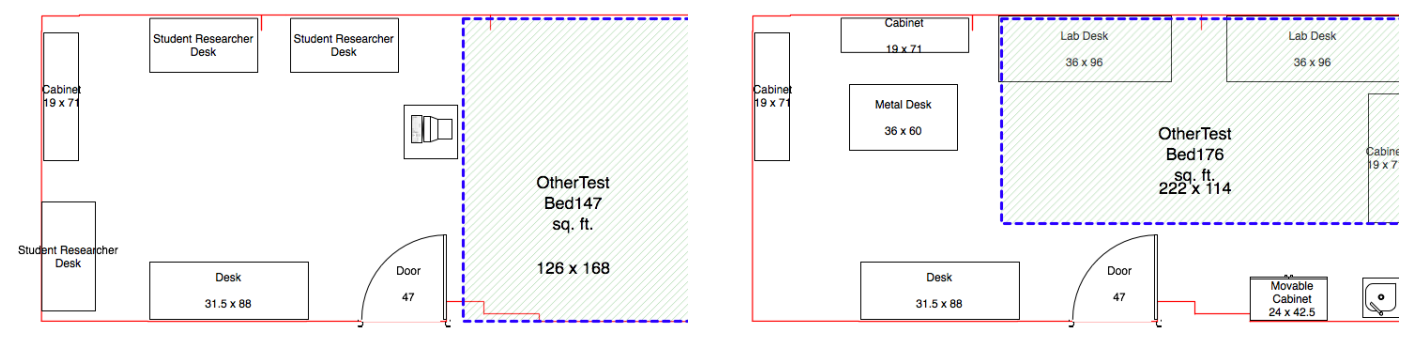

FIGURE 5-CAD drawings of two possible configurations for the laboratory space. The first layout was chosen because it more efficiently utilized the space.

tion began. Whether the board was dusty or clean, it still provided a considerable amount of friction for the robot's wheels. The same was true when the test was repeated for dirty robot wheels.

In order to protect the underlying anti-static tile, a sub-floor was anchored into the concrete. Eighth-inch plywood was used to construct the sub-floor, spanning the entire area of the testbed. The seams of the sub-floor were taped in an attempt to smooth the edges of each seam. The sub-floor panels were scribed to meet all of contours of the outer walls.

The white marker board sheets lay perpendicular to the sub-floor panels so that the seams of the sub-floor would not meet the seams of the marker board sheets. This would help minimize ridges where the panels met. The top floor was glued to the sub-floor to avoid using nails and potentially cracking the top surface. The edges and seams of the top floor were filled with a white caulk to prevent moisture and dust from getting under the surface. It was later found that the caulking attracted dust particles, turning the caulk a dark brown color. In order to facilitate cleaning the testbed, the caulk lines were painted flat white. This successfully prevented dust from collecting at the seams.

The rubber base around the wall was also found to collect dust and dirt. This caused the occasional pixel to threshold to red or even green, producing inaccuracies in the robot 


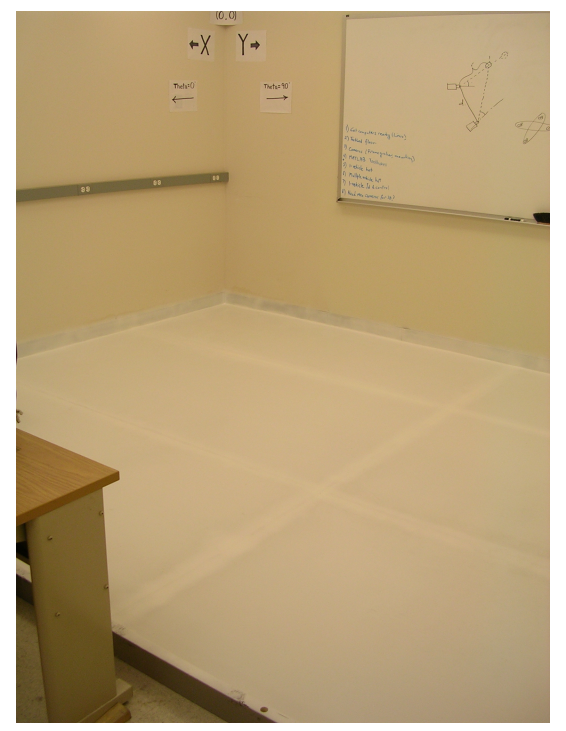

FIGURE 6-Image of actual testbed floor. The short fence is in the bottom of the image. The walls enclose the other sides.

measurements. To solve this problem, the trim was also painted flat white.

Three sides of the testbed are enclosed by walls. The fourth side is closed with a short fence to prevent the robots from accidentally driving outside the testbed. Figure 6 shows the finished testbed floor.

\section{Camera Location}

Four video cameras are mounted in the ceiling, facing downward. They are offset in a way that maximizes the floor coverage. A model of the testbed was built in Octave to simulate the placement of the cameras as well as their floor coverage. One rendering of the model is shown in Fig. 7. The height of the cameras is the main variable in this modeling problem. Once the height is determined, the placement positions of all the cameras are calculated so that the entire testbed area is covered. There is a light that spans the width of the testbed that hangs 12 inches below the ceiling. It was a potential obstruction for the cameras, so it was included in the model. From Fig. 7, the floor of the testbed is shown as four overlapping rectangles. The corners of these rectangles correspond to red lines 


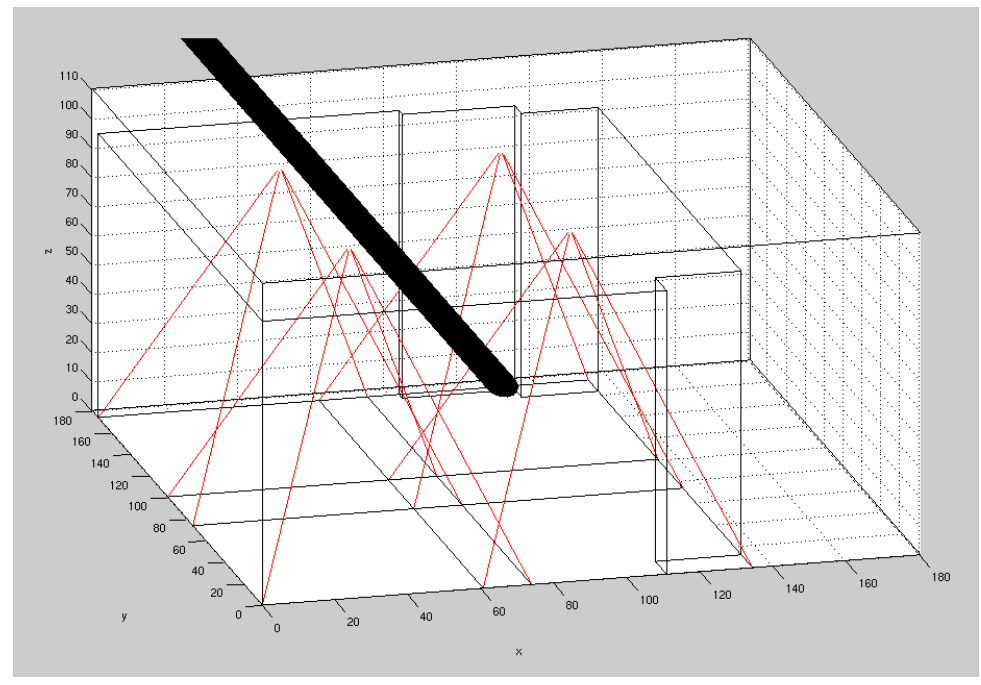

FIGURE 7 -Three dimensional model used to simulate placement of the cameras in the room. The large, black bar in the model represents the lights that hangs 12" below the ceiling. Measurements calculated from this model were used to construct the physical testbed.

extending from the cameras showing the fields of view. The angles of these red lines were found experimentally and will be discussed later with camera calibration issues. The height of the cameras could be varied, and the rectangles, or fields of view, of each camera could be analyzed. The model would also show if the hanging light would obstruct any camera view. Once an appropriate height was found, the position parameters from the model were used to place the physical cameras in the ceiling.

In the initial testbed design, the frames from each of the four cameras would be cropped and joined together to form a single large picture of the testbed. This allowed for a simpler search algorithm. In order for this to take place, the camera coverage needed to overlap, for several reasons.

First, when a robot passes between cameras, it is more effective if the cameras have a common reference. It is virtually impossible to align the cameras perfectly with respect to each other. In any practical case, there will always be some offset which creates a "schism effect". This is best illustrated in Fig. 8. Here, two robots are shown in one section of the testbed. The robot on the left is moving between two cameras and its hat pattern is altered. This could cause the algorithm to fail. The robot on the right is traveling through the center 


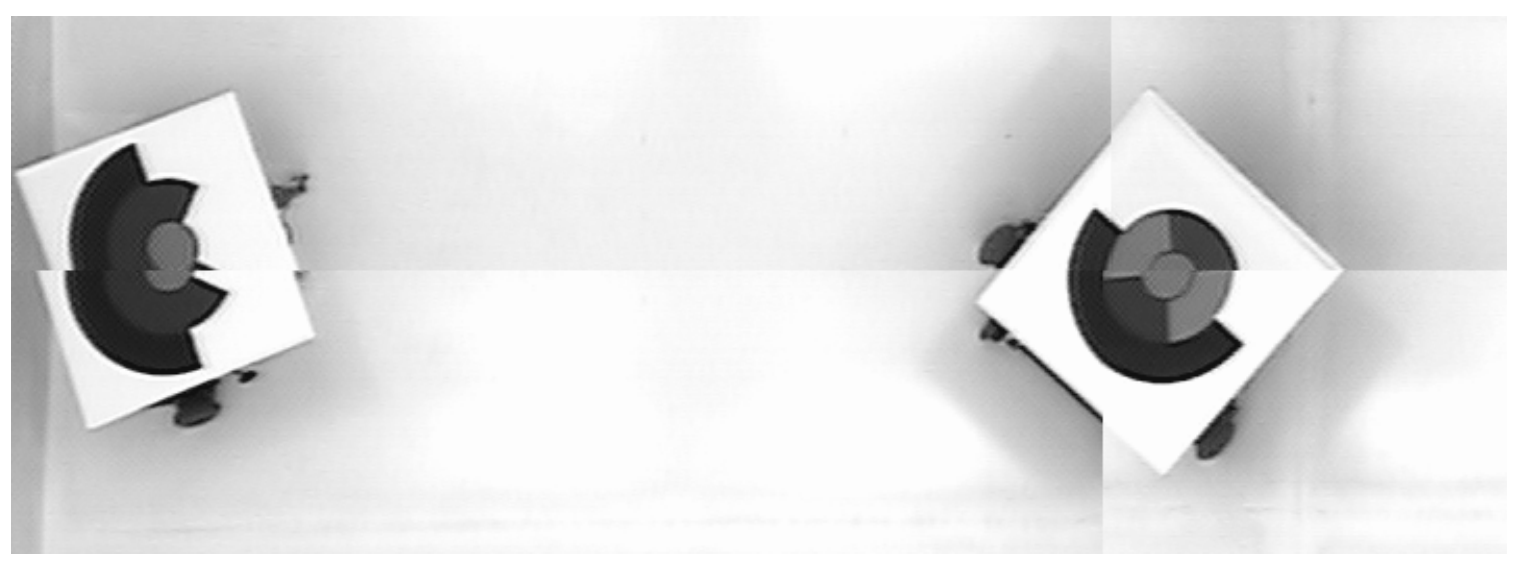

FIGURE 8-A gray-scale image of a section of the testbed with two robots. The cameras were carefully tuned so that they would combine well in the center of the testbed. The robot on the right is in the center and there is a nice image of the pattern. Although the image is nice here, that is not the case for another area of the testbed. The robot on the left shows the schism effect of lens distortion.

of the testbed. The four camera frames join here and actually produce a nice image of the center robot. Although the center is aligned well, the left side of the image is not. This introduces a more serious problem: lens distortion. Lens distortion is present in all lenses. This particular type of lens distortion is known as barrel distortion. Figure 9 shows an example of this distortion. Barrel distortion is a phenomena observed with curved lenses, where the magnification decreases as the distance from the center of the lens increases. In the figure, the grid lines should be perpendicular, but the image was taken through a common, curved lens, causing the lines to bow. The grid squares in the center of the image are slightly larger than those on the outer edges; especially the corners. Barrel distortion is more prevalent in wide-angle lenses, but even the video cameras used here, though not wide-angle lenses, produce some distortion. The distortion is most apparent in the corners of the video frame.

Unfortunately, the corners of the frames need to match when all four camera image frames are combined to form a single image. This problem can be lessened with larger overlap. It allows the higher distortion corner regions to be cropped. Areas closer to the center of the lens have far less distortion, so images can be connected much more smoothly. Figure 8 proves that there will always be a rift present when trying to join images together. 


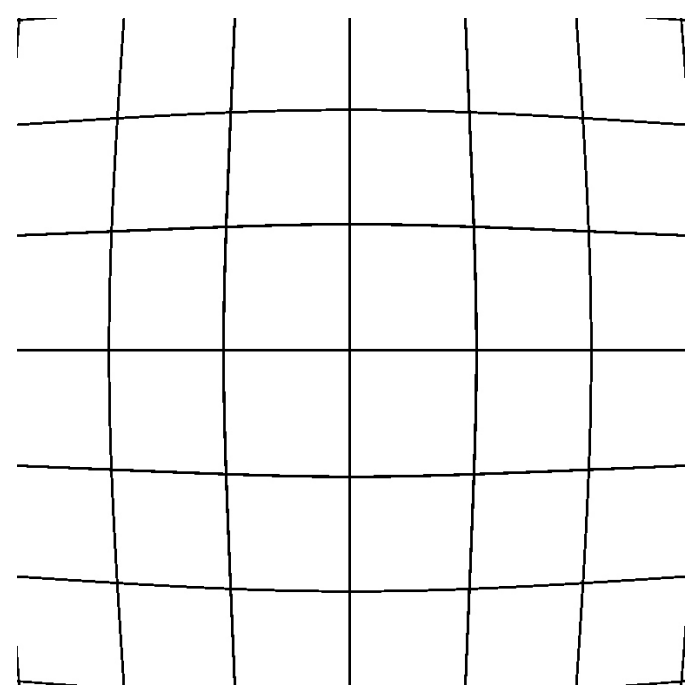

FIGURE 9-This is an image taken through a lens with barrel distortion. The grid lines should all be orthogonal. There is a difference in magnification levels between the center of the lens and the outside of the lens.

If the system is tuned for one area to match, it will cause a greater schism in another area. All of the distortion effects could be mitigated more easily if the cameras overlapped enough so that the entire robot could be seen by both neighboring cameras when traveling between regions. This would eliminate the need to join the images at all. Neighboring cameras could get a full view of the robot in the transition region. A robot's position could be calculated independently by two cameras while in the overlap region.

The model clearly showed this would not be possible unless the cameras were recessed into the ceiling. It was desirable to keep the cameras below the ceiling. So, initially they were mounted that way. Later, the lens distortion proved to be too great for the details of the multi-robot pattern. When the robot traveled between camera regions, the algorithm would fail unpredictably. The best solution was to raise the cameras to a height that would allow a robot to be completely seen by at least two cameras when traveling between regions of coverage. The model is shown in Fig. 10. Notice how the model shows the hanging light is just outside of the field of view of the cameras. This provided a maximum allowable height for the camera mounts. The ceiling height is 110 " from the floor. The camera lenses are mounted 112" above the floor. 


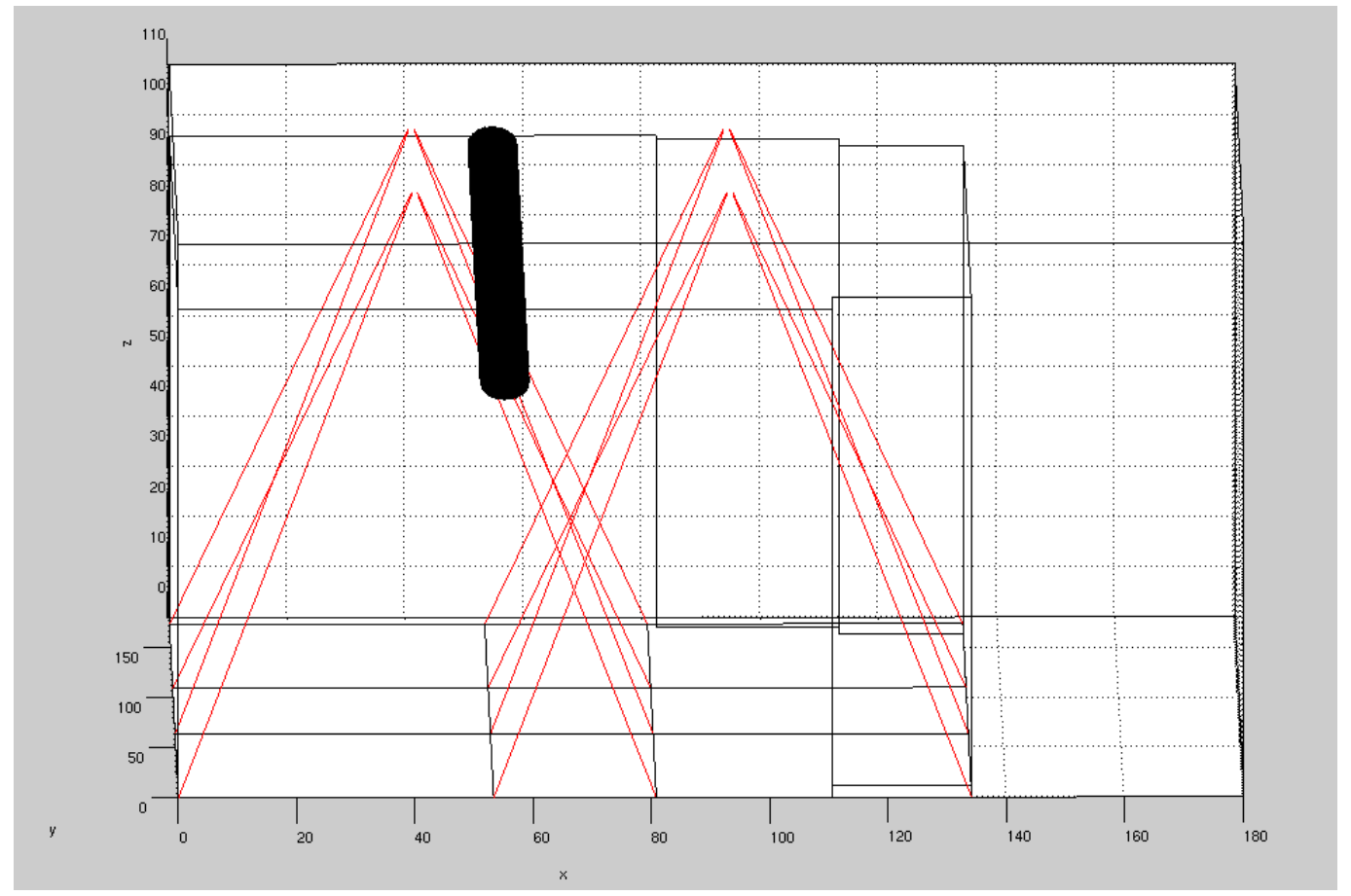

FIGURE 10 - Testbed model shown at camera height used for actual testbed. With respect to the origin shown in the model, the camera lenses are located at the following positions: (36.8", 50.0", 112.0"), (36.8", 123.5", 112.0”), (97.2”, 50.0”, 112.0”), (97.2”, 123.5”, 112.0”)

\section{Camera Mount Construction}

Although the computer model showed a fairly accurate rendering of the testbed, there will always be some disparity between the model and the actual placement of the cameras. This was taken into account when building the camera mounts. Another aspect considered was the large amount of obstructions above the ceiling. The mounting system would need to be flexible to avoid obstructions and adjust camera positions to cover the testbed floor.

The structure consists of two beams running parallel the length of the testbed. Smaller platforms span the separation of the beams. The platforms and the beams are joined with threaded rod. The long beams have a slotted hole that runs the length of the beam, allowing the threaded rod to be joined at any place on the beam. The platforms actually support the cameras. A similar slotted hole runs the length of the platform, allowing 


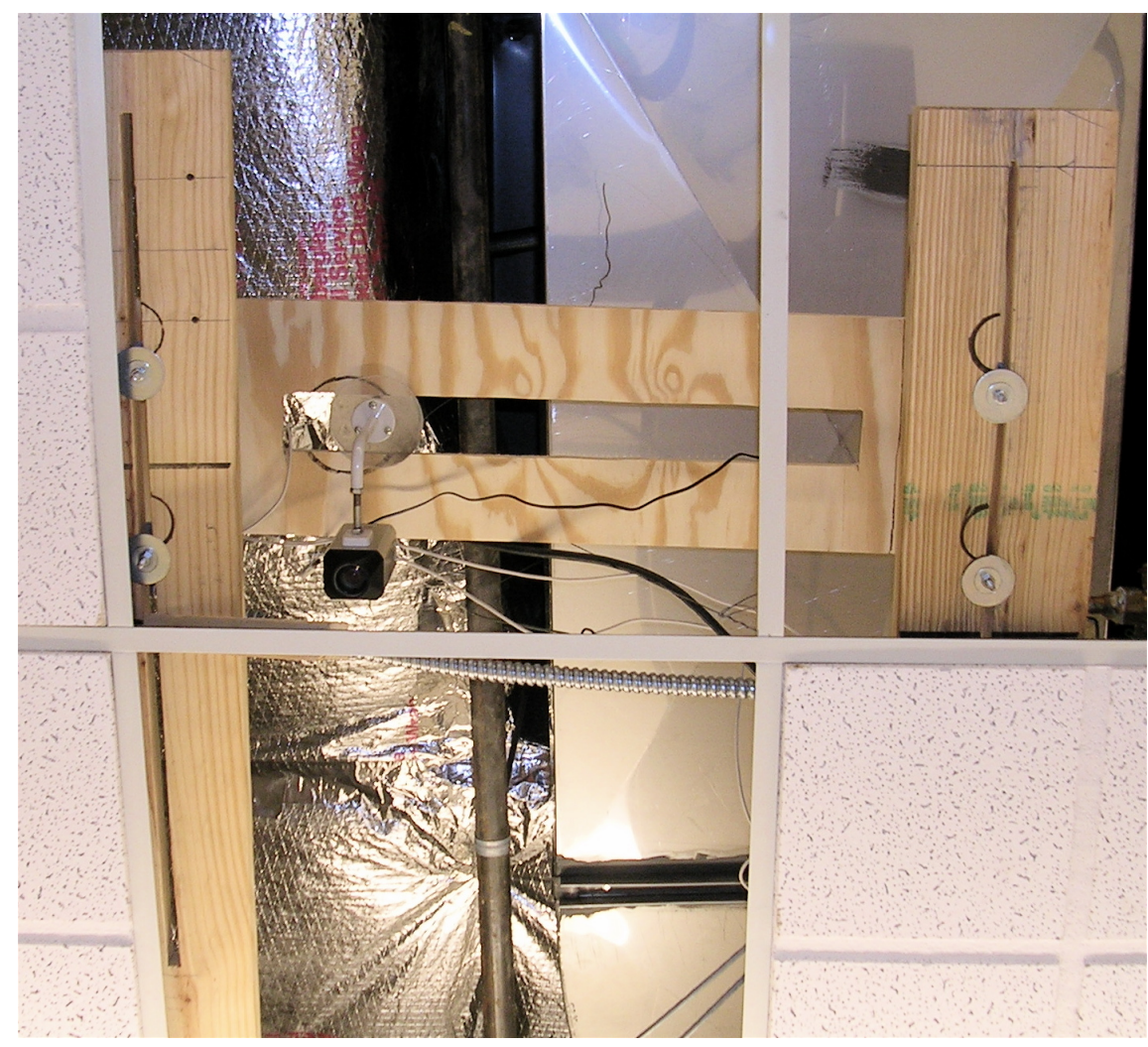

FIGURE 11 - Mounting beams used to support cameras. Slotted holes allow them to be mounted anywhere along the beam for flexibility during calibration.

the camera mount to attach at any point along the platform. These two slotted sections allow the camera to pan in the $x$ or $y$ direction. These slotted-hole sections are shown in Figs. $11 \& 12$. The threaded rod connections provide vertical adjustment for the cameras. By simply loosening or tightening a set of four nuts, the camera platforms can be raised and lowered, as well as leveled. It is important to have all of the cameras level so that perspective distortion is negated. An angled camera would produce an effect known as "converging verticals", where parallel lines appear slightly angled in the image. This is most apparent with much larger distances, however, care was still taken to avoid it.

Consideration was also given to rotation. The camera L-joints are joined to the platforms by two discs that "sandwich" the platform. The discs are bolted together in the center, which can be seen in Fig. 13. When loosened, the discs can slide any way along the slotted hole, providing the panning motion described previously. The discs can 


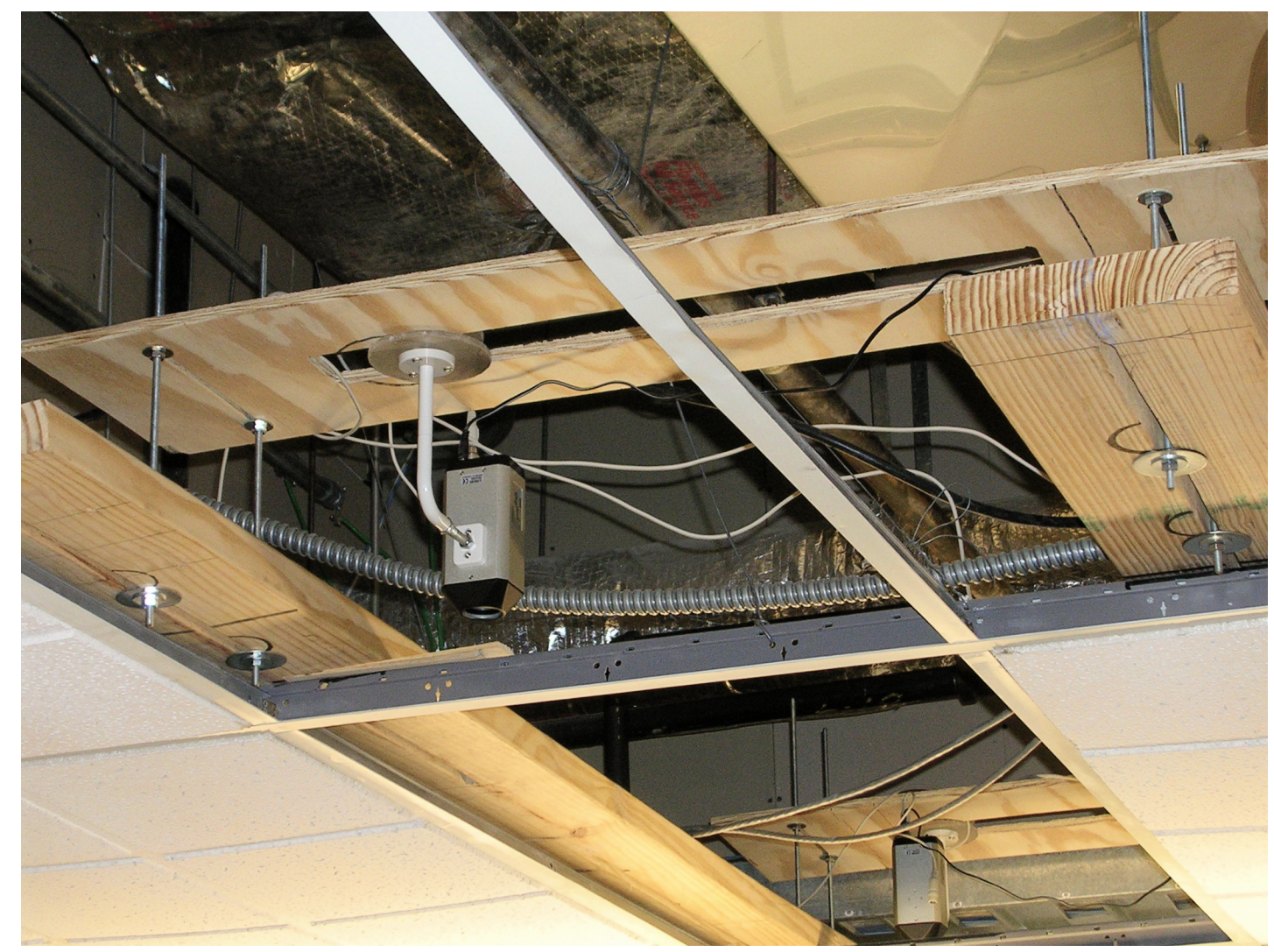

FIGURE 12 -Mounting platforms that hold cameras. Slotted regions allow the cameras to slide anywhere along the platform.

also be rotated, allowing the camera to be rotated squarely with the floor. Two cameras share two long beams, with their respective platforms spanning them at opposite ends. Figure 14 shows the final assembly with the cameras in place. With this configuration, the cameras can see the entire robot as it passes between camera regions, so there is no need for combining distorted images. This figure shows two pictures of all the testbed camera mounting hardware.

\section{B. Software Implementation}

The language chosen to implement the tracking algorithms is $\mathrm{C}++$. This was chosen for its compatibility with known drivers, as well as robotics software. The source code for the single and multi robot algorithms is located in Appendix I and II, respectively. The 


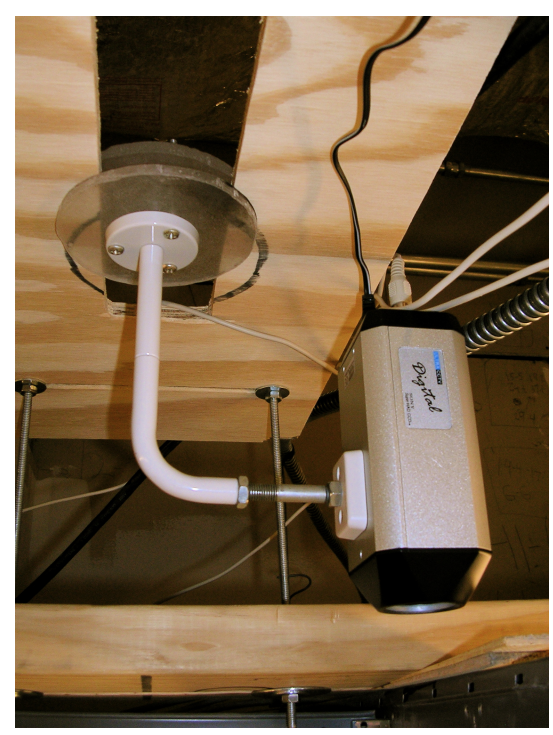

FIGURE 13 -Close-up of mounting brackets or discs used to attach L-arm to mounting platform. The discs sandwich the platform, providing a solid base while still allowing the cameras to rotate, if needed.

algorithms presented previously do not differ with those actually implemented. However, there are a few implementation details worth mentioning. The problems encountered along the way, along with the solutions, are also discussed.

\section{Efficient Enhancements}

Two common features of both the single and multi-robot algorithms greatly improved the speed of the robot searches. One feature was the thresholding method. Typically, there is a sequence of "If Greater Than Low Level And Less Than High Level" statements that determine whether a pixel gets classified as a particular color. For color images, there are three of these If-type statements for every color. This is not very efficient, especially as the number of colors increase. This can quickly become a time consuming process.

Another method involves using lookup tables for threshold values. Three vectors are used to represent three color channels. The ranges corresponding to an acceptable level are assigned a "1", while the ranges not associated with the color are " 0 ". Figure 15 shows 


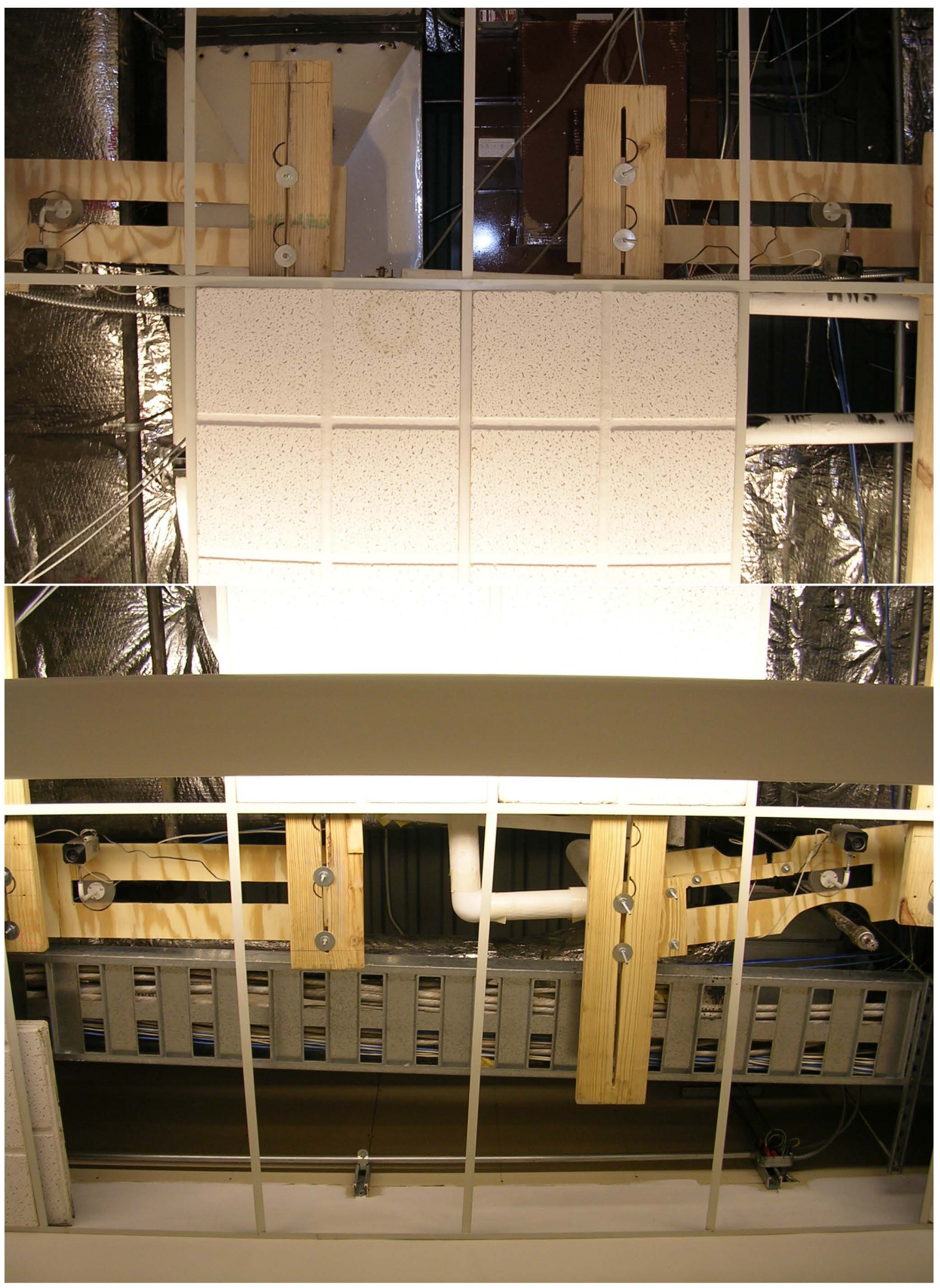

FIGURE 14 - View of all the camera mounting hardware for the testbed. 

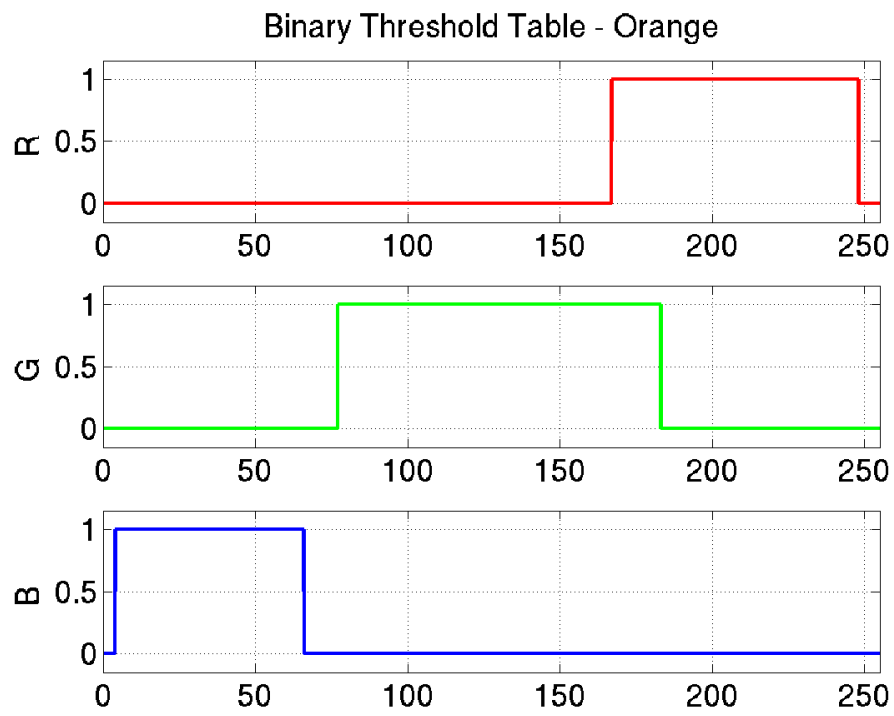

FIGURE 15-Example of a thresholding lookup table for the color orange in the RGB color space. 1's represent an acceptable match level. If the lookup table returns three 1's, the pixel will be classified as orange.

an example threshold table for the color orange. In the Red-Green-Blue (RGB) color space, orange is represented by a mixture of red and some green. Color values are typically 8 bits, which yield $2^{8}$ or 255 different levels. So, the threshold tables have a length of 255 . Higher values of the red channel are assigned "1", along with the mid-level green values. Orange has very little blue content, so "1's" are given to the lower blue levels. The thresholding is performed using the pixel vector as the indices to the thresholding tables. The result of the three look-ups will be a mixture of zeros and ones. These results are ANDed together to give the final result. If the pixel vector finds a "1" in all three lookup tables, the pixel is a match to the color orange. If one of the pixel components finds a "0", the pixel will not be classified as orange. This algorithm has been found to drastically reduce computation time for thresholding an image [9].

The typical compiler assigns 32 bits for an integer. The lookup tables only require a single bit in each of them to represent a color. With integer lookup tables, 32 color threshold levels can be stored. One thing to note with this method is that overlap of colors in the lookup table is possible. One pixel could be classified as being red or orange if there is 

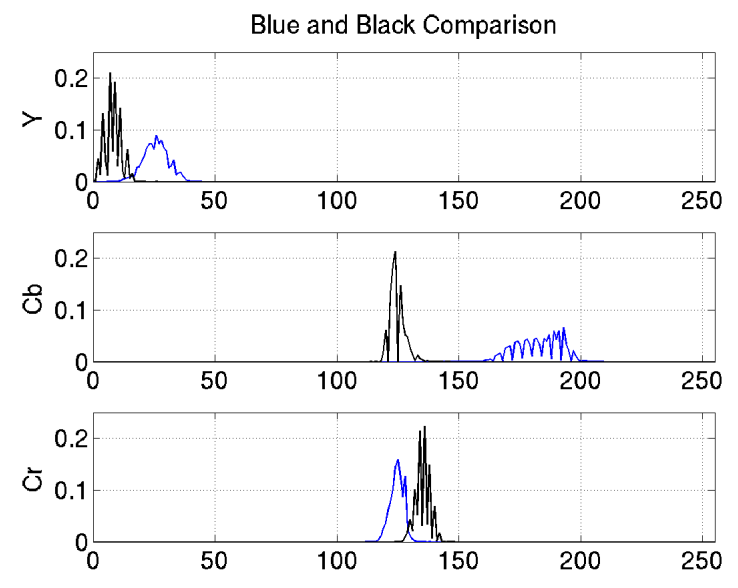

FIGURE 16-Histograms of the colors blue and black in the $\mathrm{YCbCr}$ colorspace. Notice the overlap in the $\mathrm{Y}$ and $\mathrm{Cr}$ channels. There is also little separation in the $\mathrm{Cb}$ channel, making these two colors difficult to distinguish, especially in changing lighting conditions.

overlap in the lookup table. This is an acceptable situation, because the color patterns were carefully selected in order to maximize the contrast between colors in the same feature.

For example, blue and black are very closely related according to threshold levels. Figure 16 shows histograms of the $\mathrm{YCbCr}$ components for the two colors. The $\mathrm{YCbCr}$ or YUV colorspace is another way to represent color and will be discussed in more detail later. Notice that there is some overlap, particularly in the $\mathrm{Y}$ and $\mathrm{Cr}$ component. Blue has a slightly higher, though not always distinct, gray-level. It also usually has a higher blue component. When there is noise present in the environment, blue and black become difficult to separate. However, blue and black are never used in the same feature. Black and white are used for the orientation measurement. Blue, green and white are used to encode the robot number. So, classification error due to the overlap of black and blue threshold regions will not cause the algorithm to fail.

The second beneficial commonality of the single and multi-robot algorithms is the use of sub-sampling during the image search. This method is used successfully in [7]. Instead of analyzing every single pixel in the image, varying numbers of rows and columns are skipped. Whenever a critical color is found, such as red or blue, a more detailed search 


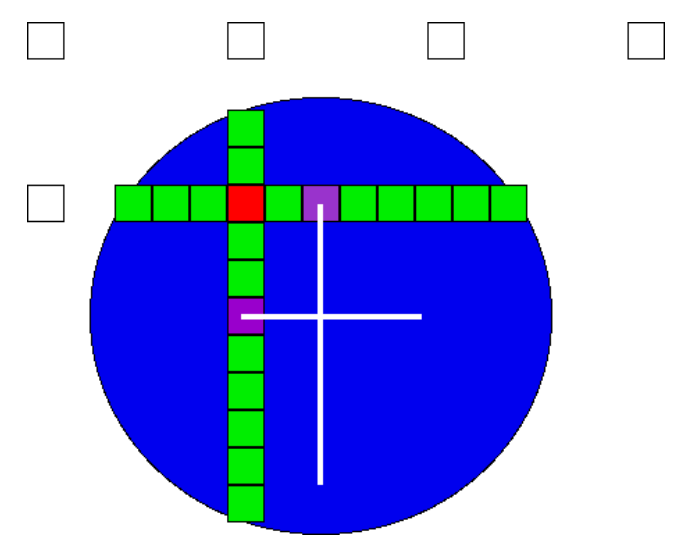

FIGURE 17 - Search method and center calculation used in [7]. The sub-sampled search method was used for the local testbed, but a different algorithm was chosen for pose calculations. White squares indicate not finding the blue circle. Red square indicates a "hit" from the search for blue. Green pixels are measuring chords of the circle. Purple squares are the center of each chord. White lines show perpendicular bisectors, which intersect at the center of the blue circle, a.k.a. the center of the robot.

is performed for that area of the image. In [7], they use a pattern very similar to the pattern used in this multi-robot algorithm. All hat patterns developed have the same features: 1) An area to locate the center of the robot 2) Some sections to encode a robot ID number 3) A feature to find the orientation. Although the patterns are similar, the algorithms are quite unique. The method used in [7] uses a sub-sampled search, but a entirely different method is used for finding the robot's center. The centroid calculation used here is illustrated in Fig. 17.

The white squares indicate the sub-sampled search from left to right, top to bottom. The pattern used here searches for a blue central circle, which is shown in the figure. The red square indicates a "hit" from the sub-sampled search. Now, the search expands to the north, south, east and west, as shown by the green pixels. This search stops when the edges of the blue circle are found. The green lines are perpendicular chords of the blue circle. Perpendicular bisectors of the chords, shown as white lines in the figure, will intersect at the center of the blue circle. This gives the center of the robot.

Although this method requires a search of very few pixels, it was not the method chosen to track the robots for this testbed. This approach is very fast, but is highly susceptible to noise in the environment. If a single pixel in the north-south or east-west line is 
misclassified, the centroid calculation will be inaccurate.

Instead, once a "hit" is found during the sub-sampled searching, a thorough neighborhood search is performed and the center of mass is calculated as described by equation 5. If a single pixel is misclassified, the centroid calculation does not suffer. Figure 18 shows a visual representation of this method for the single robot hat pattern. The white pixels in the figure are not analyzed for color content. They are assumed to belong to the background. The black dots show which pixels actually were measured for color content. They were set to black when they did not threshold to red or blue. The search continues across the image until a red or blue pixel is found. There is a large section of black pixels around the robot in the image. This is a result of finding one of the red pixels in the image. The black box represents the neighborhood of pixels searched after that event occurred. Some of the pixels in the black box thresholded to blue and some to red. The centers of mass were calculated for these two blocks. Green dots were placed at those centers of mass. The final position is the result of averaging the red and blue centers, as discussed previously.

The searching box can be varied in size, but must be large enough to encapsulate the entire robot. For the single robot algorithm, the search box is large because it must encompass the entire hat. The box is smaller for the multi-robot hat because all that needs to be found is the small red circle. Also, the number of rows and columns that are skipped changes between the algorithms. It must be kept small enough to be sure to detect at least one critical color pixel. For the single robot, the red and blue blocks are quite large, so more rows and columns can be skipped, saving time. However, the multi-robot pattern contains a smaller critical area, so fewer rows and columns are passed over in order to guarantee detection. 


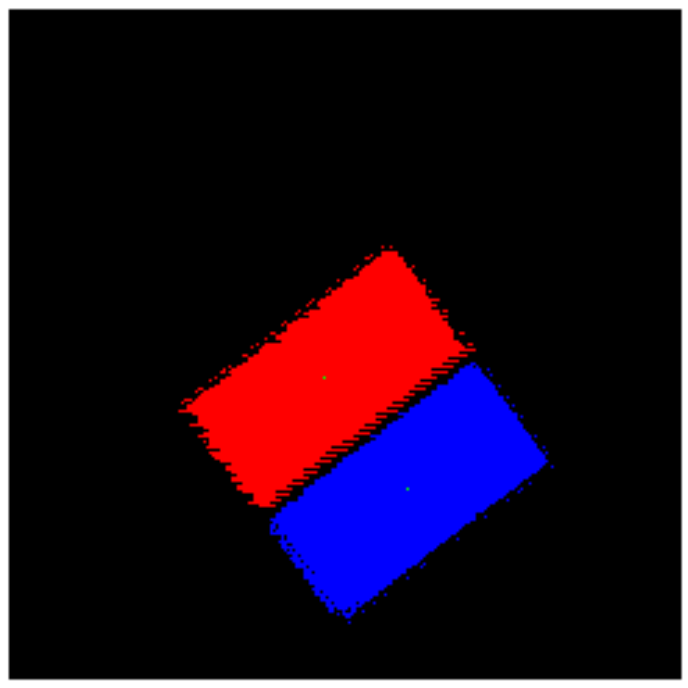

FIGURE 18 - Visual representation of the sub-sampled search algorithm. White represents pixels that were not examined. The black dots show what pixels were thresholded. The black box shows where the refined area search took place. This is the search for the single robot. The green dots represent the centers of the red block and the blue block. Notice that the algorithm stops searching once the single robot is found. 


\section{Challenges}

Between the two algorithms, the single robot and multi-robot, several challenges arose. The first implementation of both of these algorithms fell far short of expectations. While both accurately measured the pose of the vehicles, the measurement frequency was a mere $1.5 \mathrm{~Hz}$. This failed to meet the real-time need of the testbed.

After further analysis, it was found that both programs spent most of the time waiting for the image data to arrive to memory for processing. The robot search algorithms operated a scant $8 \%$ of the running time. The other $92 \%$ was spent waiting for the framegrabber to capture an image. The frame-grabber purchased was capable of incredibly fast capture rates, however, the problem was the software driver.

After much investigation into the workings of the driver, it was discovered that the frame data went through a time-consuming color-space conversion. The native output format of the cameras is YUV, a color space consisting of gray-scale (Y), blue component (U) and red component (V) channels. The driver was converting the YUV channels to Red-Green-Blue (RGB). Then, it performed a second conversion from 4-bit RGB values to 8-bit RGB values. Once an image was captured, the pixels were converted to two different color-spaces. Only then could another image be captured.

At this point the search algorithm could begin, but too much time was already lost. The end result was a sluggish tracking rate of $1.5 \mathrm{~Hz}$. So, a new driver was selected that would capture the frame data more efficiently. This new driver had the option to work in the native format of the camera, YUV. The driver was modified slightly to get even faster performance. The output directly from the camera was in a compressed video format known as YUV 4:2:2. This format is similar to that used by high-definition televisions. The frames were analyzed in this raw, compressed YUV 4:2:2 format to greatly improve the measurement frequency of the testbed. As a result modifying the frame-grabber drivers, the tracking rate surged from 1.5 to $30 \mathrm{~Hz}$.

The second major challenge was mentioned earlier with the camera mounting. The 
lens distortion of the cameras prevented the multi-robot algorithm from measuring robots in the overlap region. The single robot hat pattern is large and simple. Small schisms or rifts in combining four camera frames together did not affect the end result of the simple algorithm. The mere size of the simple hat features made it robust enough to handle well.

The multi-robot hat pattern contains more information. In order to keep the pattern roughly that same size as the single robot pattern, the feature size was reduced. This magnified the lens distortion's effects. Suddenly, the search algorithm would find a 70 degree shift in the robot's orientation simply because the images from neighboring cameras had some sort of rift.

This was not a problem that some amount of calibration could correct. Even if cameras were calibrated to provide perfect matching in the center of the testbed, the outer edge of the testbed would show the distortion effect. This would cause the multi algorithm to fail. The only solution was to raise the camera height. Using the model shown previously, the cameras were raised from a height of 92" above the floor to 112". The overlap area increased enough so that the need to combine frames was eliminated. 


\section{CHAPTER IV CAMERA CALIBRATION}

Several problems arose while working with the cameras. In practical applications, a camera, like anything else, is imperfect. Lens distortion, though small, proved a formidable challenge in the implementation of this testbed. That problem, discussed previously, was solved by raising the height of the cameras. Another problem was reconciling the differences in color levels between each camera.

Although the four cameras are identical in make and model, they still do not respond exactly the same way to light. The charge-coupled device (CCD) sensors employed on the cameras, though similar, are not exactly the same. There are several parameters on the cameras that can reduce these differences such as color gains and the aperture setting. However, it was still not possible to get identical color levels from the four different cameras.

Another difficulty encountered dealt with color thresholding. Color thresholding is a process of classifying a pixel as a particular color. There are very complex and time consuming methods of pattern classification [10]. Typically, the more advanced the method, the more costly it is in terms of speed. One of the major goals of this testbed was to operate in real time. So, a great effort was made to separate the colors as much as possible.

The camera hardware settings and color thresholding will be discussed in detail later in the chapter. The first feature studied is a simple one. The object of using overhead cameras was to track robots on the testbed floor. In order to know how much area a single camera could cover, the field of view of the camera was measured. 


\section{A. Field of View}

The ultimate goal of using the cameras was to sufficiently cover the testbed floor and track vehicles, wherever they might be. So, the field of view of a single camera was calculated experimentally. This was done by taking measurements of the camera's cone angle. Light enters the lens at an angle and is refracted to a focal point. A rectangular array of light sensors is placed between the focal point and the lens, sensing the incoming light. Any light entering the lens greater than a specified angle is not refracted through this system and therefore unseen by the image sensor.

This angle is referred to as the field of view or cone angle. This angle was measured experimentally at specific distances from the camera lens along the horizontal plane of the camera, as well as the vertical plane. The camera was placed on the floor, centered on a straight line. At a distance of 10 inches from the lens, an object was placed on the floor, outside the camera's view. Using live video from the camera, the object was moved toward the center line. When the object was seen by the camera, the perpendicular distance from the center line was recorded. This process was repeated in 10 inch increments to 70 inches. This was also done for the opposite side of the center line in the horizontal plane. The angle found was symmetric about the center line. The total field of view in the horizontal plane measured $52.79^{\circ}$.

The camera was then rotated 90 degrees to take measurements in the vertical plane. The process was repeated. It was discovered that the vertical angle was different from the angle in the horizontal plane, at only $40.16^{\circ}$. This is expected because the CCD sensor is not square. There are 640 lines of resolution that span the horizontal axis, while only 480 cover the vertical axis. These measurements were plotted in three dimensions. Figure 19 shows this plot. The red circles represent the data points in the vertical plane. The blue represent the horizontal plane measurements. Using the least squares method, lines of best fit were found and plotted as green lines. The final camera coverage is shown as the green rectangle at a distance of 110 inches from the camera lens. These measurements were used 


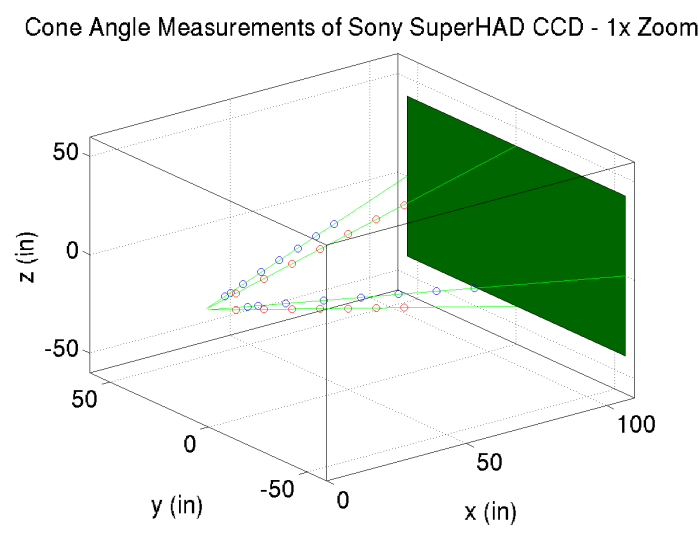

FIGURE 19-3D view of cone angle measurements taken from video camera at 1x zoom. Red points represent measurements taken in the vertical plane. Blue points are measured in the horizontal plane. Green lines show least squares fit. Rectangle shows field of view at a distance of 110 ".
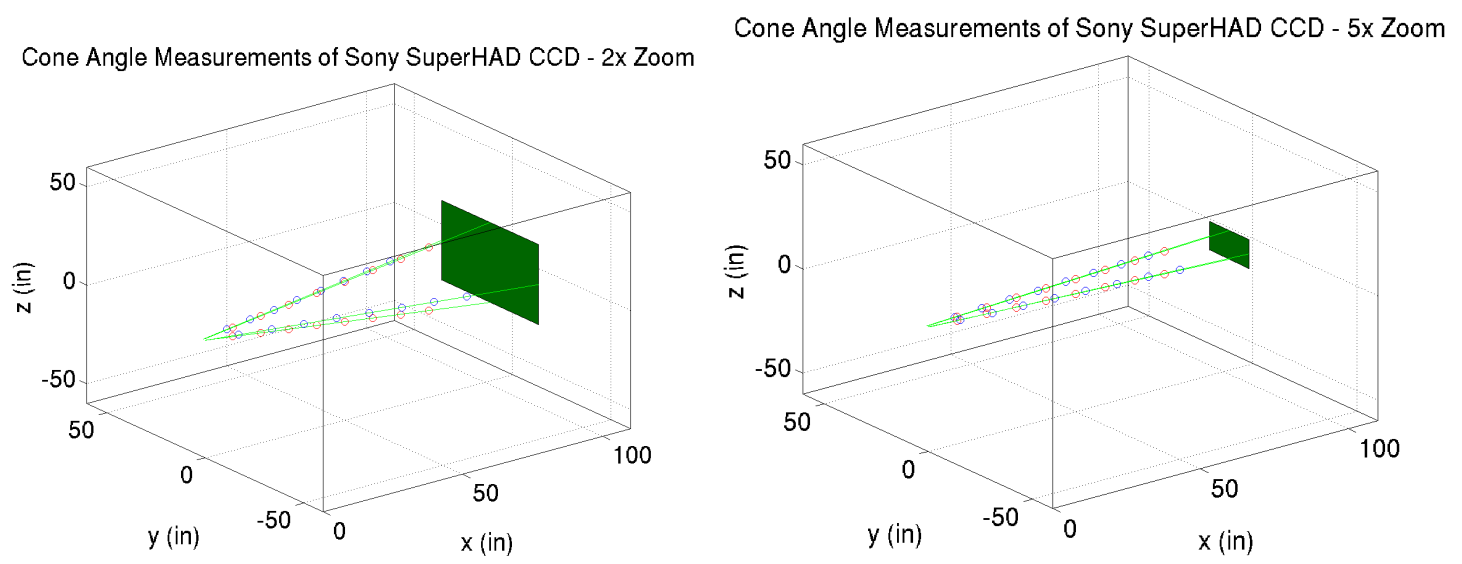

FIGURE 20-3D view of cone angle measurements taken from video camera at $2 \mathrm{x}$ Zoom on the left, 5x Zoom on the right. Red points represent measurements taken in the vertical plane. Blue points are measured in the horizontal plane. Green lines show least squares fit. Rectangle shows field of view at a distance of $110 "$.

to develop the testbed model seen previously in Chapter III. The measurement process for 1x zoom was repeated for another camera and the results were indistinguishable at the level of measurement precision. So, the model here was applied to all cameras.

These measurements were also repeated for different zoom levels of the camera. The original experiment was at $1 \mathrm{x}$ zoom. The next two experiments were done at $2 \mathrm{x}$ and 5x zoom. The results are plotted in Fig. 20. Notice how the zoom affects the field of view. The angles are decreased as the zoom increases, allowing less area to be covered. 

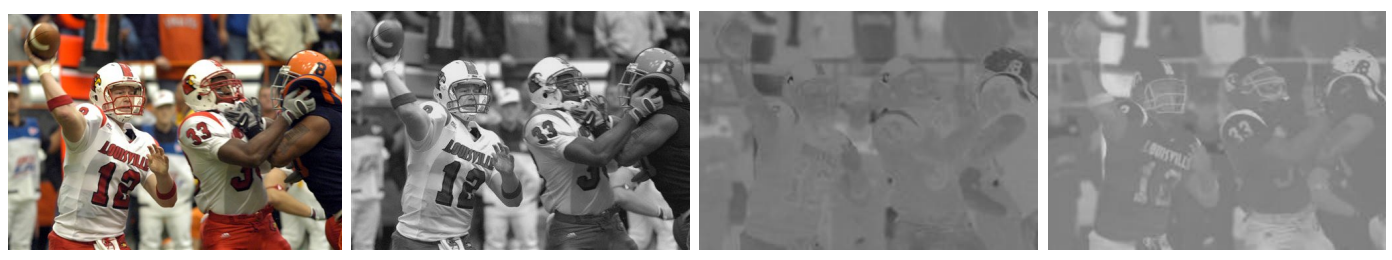

FIGURE 21 - Picture of Cardinal quarterback Brian Brohm throwing a pass against the Syracuse Orangemen. Used here to illustrate the $\mathrm{YCbCr}$ color space. Left, is the original image. The images to the right of the original are the $\mathrm{Y}, \mathrm{Cb}$ and $\mathrm{Cr}$ represented as gray-scale images.

\section{B. Multi-Camera Color Consistency}

Although the cone angles of the four cameras matched well with each other, some of the other features did not. Of the twenty or so adjustable settings on the cameras, only three of them proved to have a major affect on the output: aperture, red gain and blue gain. The native output of the cameras is YUV or, once digitized, is also known as YCbCr. This is known as component video and is a widely used format in video processing [11].

\section{A Word About Color Spaces}

The Y component represents the gray-scale or intensity component of the image. It is simply the black and white image. The other two channels contain the color information of the image. They are represented as the blue component, $\mathrm{U}$ or $\mathrm{Cb}$, and the red component, $\mathrm{V}$ or $\mathrm{Cr}$. This is more apparent in Fig. 21, where a picture is shown, along with the three $\mathrm{YCbCr}$ channels alongside the original image. The red in the image has a high red component value. So, red shows as near white in the $\mathrm{Cr}$ image. The dark blue jersey of the defensive tackle contains a mid level blue component. That jersey appears mid-gray in the blue component image. The human eye is more sensitive to the intensity information than the color information in an image. As a result, the color channels, $\mathrm{Cb}$ and $\mathrm{Cr}$, are usually sub-sampled in order to compress the video with little psycho-visual loss.

The format of YUV 4:2:2, means that for every four gray-scale pixels, there are only two $\mathrm{Cb}$ and $\mathrm{Cr}$ pixels. Therefore, the size of the file is reduced to half of the original 
size.

There are other reasons that the $\mathrm{YCbCr}$ color space is chosen in computer vision research. Changes in lighting conditions wreak havoc on computer vision algorithms. Color levels shift when the amount of illumination in the room changes. This forces thresholding levels and comparison images to change, usually causing the algorithm to fail. The $\mathrm{YCbCr}$ space is different from the RGB space in its susceptibility to lighting changes.

If illumination decreases, an image in the RGB space will see a decrease in levels in all three channels, red, green and blue, alike. In the $\mathrm{YCbCr}$ space, lighting usually only affects the Y component. The color-components are not altered. This makes the $\mathrm{YCbCr}$ space a very popular choice for vision algorithms. Although the lighting conditions can be carefully controlled in this instance, it is always desirable to be as robust as possible. For this reason, coupled with the fact that it is the native output of the cameras, the $\mathrm{YCbCr}$ color space was chosen for the testbed camera system.

Once the color space was chosen, the process of calibrating the cameras and threshold levels began. In order to ease the thresholding process, it would be nice if all cameras viewed the environment the same way. In other words, the color red would appear red in all four cameras. The color white would be consistent among all four cameras. This color consistency problem proved more difficult than it sounds, because each camera sensor has a variation built into it. This problem has recently attracted other researchers [8].

The process of setting the cameras and setting color threshold levels are closely linked. The process consisted of making changes to camera hardware settings and then examining the affects the hardware changes had on color levels. A standard print was made, shown in Fig. 22, consisting of true red, blue, green, black and white blocks. The cameras, already mounted in the ceiling, were each adjusted to a zoom of $3 x$. The print was placed in the camera's field of view on the testbed floor. The calibration print filled the view of the camera as seen in the figure. A frame of the calibration print was captured for each camera. Then, color histograms were calculated for each camera. The approach was 


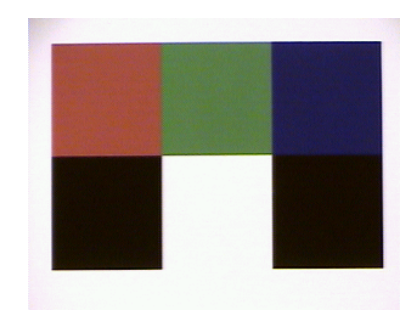

FIGURE 22 - Frame captured from a video camera of the image used to calibrate all cameras. Print consists of a block of pure red, blue, green, black and white.

to set the camera hardware so that color histograms from each camera matched as closely as possible. The three hardware settings mentioned earlier that affected the output the most were the aperture, red gain and blue gain. These three features also closely corresponded with the YCbCr color space. The aperture affected mainly the Y component. The red and blue gains primarily affected the $\mathrm{Cr}$ and $\mathrm{Cb}$ components respectively. There was some cross-correlation between the aperture setting and the color components. As the aperture decreased, allowing less light in, the color component histograms narrowed. This makes sense because as an object gets darker, less of its color information is conveyed. It is more difficult to identify the color of something in low light situations. This was, however, the worst of the coupling during calibration.

A benchmark camera was selected after iterative experiments of examining the color histograms and modifying camera settings. In order to quickly threshold colors, it is desirable that they be linearly separable. The camera hardware settings caused shifts in the color space, changing the way colors appeared, and in the end, classified. If there is a large separation between two colors in the color space, it will be very easy to classify the two of them correctly. As the separation decreases, the chance of misclassifying a pixel grows. In an attempt to minimize this, a hardware setting was found that separated the primary colors, red, green, blue, black and white, as much as possible. The histogram of this benchmark is shown in Fig. 23. This is a histogram of the camera looking at the calibration print. The job then became a process of modifying the other camera's hardware settings to produce a matching, or closely matching, histogram. This is similar to the approach taken in [8], 


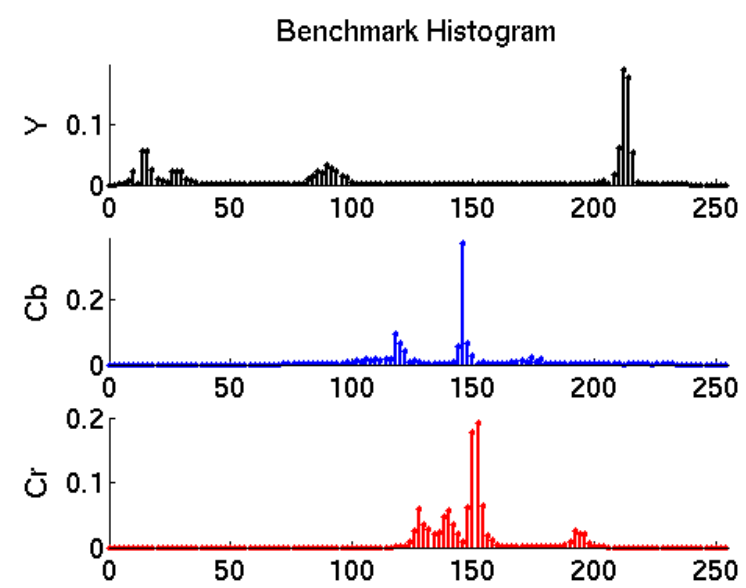

FIGURE 23 - Histogram of calibration print, taken from Camera 1, with desirable hardware settings. This provided the most separation between colors in the $\mathrm{YCbCr}$ color space.

with the exception that their process is automated. Automation requires more hardware to control the camera settings via software. Even with the automated approach, they conclude that there could never be a perfect match by merely adjusting hardware settings [8]. To achieve consistency, image processing correction is needed. The testbed camera system avoids any software correction to ensure fast tracking of the robots. The final histograms of the other three cameras are shown in Fig. 24. Notice how closely the histograms match.

\section{Color Threshold Calibration}

Once the proper adjustments were made to the camera hardware settings, the task was then to find proper threshold ranges for each of the critical colors: red, blue, green, black and white. These are the colors used in the hat patterns. Therefore, identifying them correctly is crucial.

Using the program shown in Appendix IV, a frame was captured from each camera while viewing the calibration print. Those frames were then processed interactively using the listing in Appendix V. The different color regions were identified. The mean, variance, minimum and maximum were calculated for each primary color in each of the color space channels, Y, Cb and $\mathrm{Cr}$. The program also plotted histograms in three different 

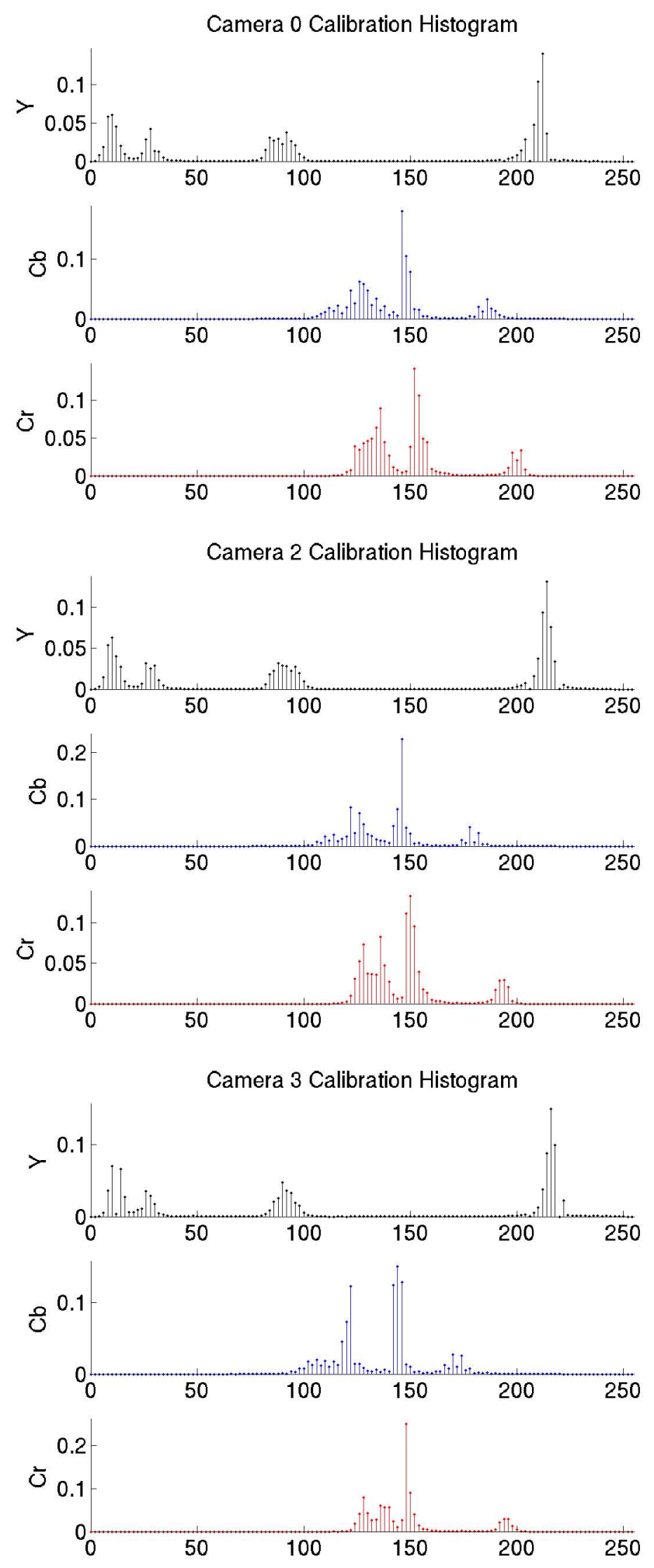

FIGURE 24-Histogram of calibration print, taken from Cameras 0, 2 and 3, to match the benchmark of Camera 1. The histograms match very well with that shown in Fig. 23 

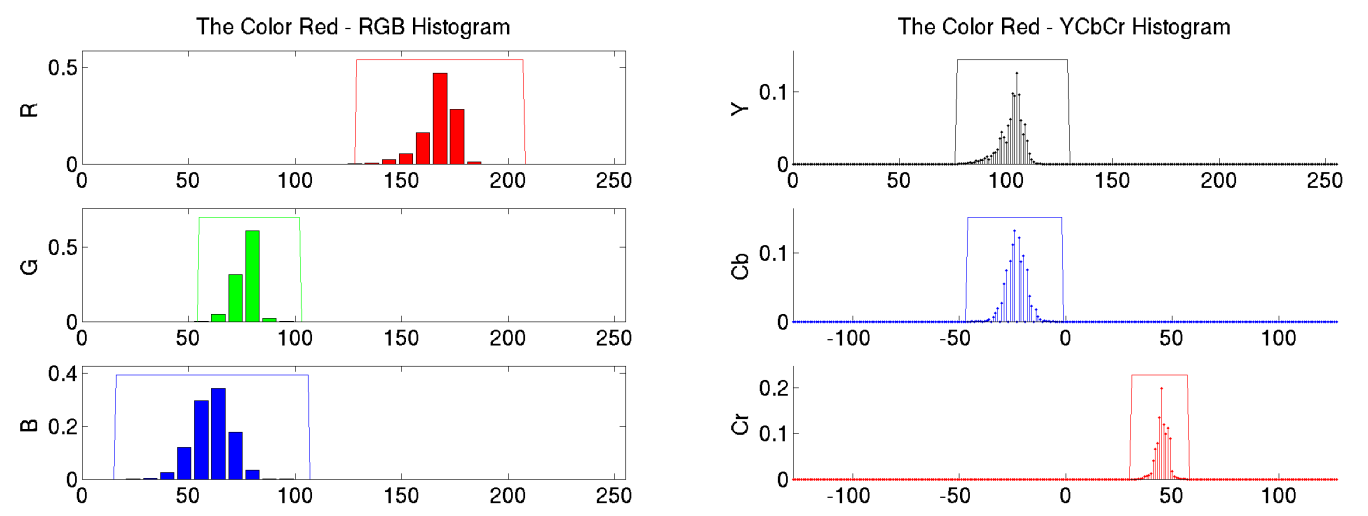

FIGURE 25-Histograms of the color red in the RGB and YCbCr spaces, taken from Camera 0, in order to determine proper thresholding range for Red.

color spaces: the common RGB space, the HSI space, and the already-mentioned $\mathrm{YCbCr}$ space. In this way, thresholding could possibly be performed in any of these spaces. The resulting histograms from Camera 0 of the color red are shown in Fig. 25 in the RGB and $\mathrm{YCbCr}$ spaces. The RGB was not chosen as a color space for thresholding because of its susceptibility to lighting conditions as well as its need for conversion from the original format.

Another color space was investigated: the Hue-Saturation-Intensity (HSI) space. The HSI space was developed as a way to describe colors the way humans do. The first channel, Hue, is the color. The second channel, Saturation, describes the fullness, or tint, of the color. The final channel is meant to describe the intensity or gray-scale value. Unfortunately, the HSI space did not offer any advantages for color segmentation, such as large separations in different colors. The HSI space also requires a more complex, non-linear space conversion. This would be too costly in terms of speed to yield any great benefit. Thus, the native $\mathrm{YCbCr}$ format was chosen for thresholding.

The samples of red, green, blue, black and white were analyzed for each individual camera. Then, the samples from individual cameras were combined to give a global threshold range for each color. The results for the global thresholds for each color are shown in Fig. 26. Bounding boxes are shown around the histogram data to indicate a recommended 
threshold range. In order to visualize the separation among all of the colors, the bounding boxes are plotted together in Fig. 27. The white range is shown as yellow on the plot. In order to easily separate two colors, they must differ in range in at least one channel. For example, red and green have some very similar characteristics. They overlap in the intensity channel and also have a similar blue content. However, in the red component channel, red is significantly higher. Red, for obvious reasons, has the highest red content of any other color, and by a significant amount. This is what makes the color red so easy to threshold without error. The tracking algorithm relies on the center color being classified correctly. That is why the center color for the multi-robot hat pattern was chosen to be red. The center color triggers the rest of the tracking algorithm. The color white is another example of a color that is easy to threshold. The separation of white from the other colors in the Y channel makes it an ideal choice for the flooring. It is, therefore, simple to separate a background pixel from a one belonging to a robot.

The threshold levels shown in Fig. 27 are those calculated by the calibration program as \pm 3 standard deviations from the mean. According to the "68-95-99.7 rule" commonly used in statistics [12], this region of a normal distribution should encompass $99.7 \%$ of the pixels in each color. This gave a good starting point, however, the threshold levels had to be widened slightly for the testbed. This is due to the lighting changes as the robot moves around the testbed. The samples were taken from the center of each camera's view, where the calibration image was placed. The lighting tended to be brighter near the center of the testbed and slightly darker near the walls.

This broadening of the threshold ranges caused some problems for classifying black and blue. These two colors are closely related. The largest difference between the two of them is the blue component. However, the variance of the color blue in the blue component channel is fairly large. There is overlap in the blue component and the intensity channels, as well as the red component channels. This overlap leads to error in classification. This problem is overcome by carefully designing the hat patterns and search algorithms so that 

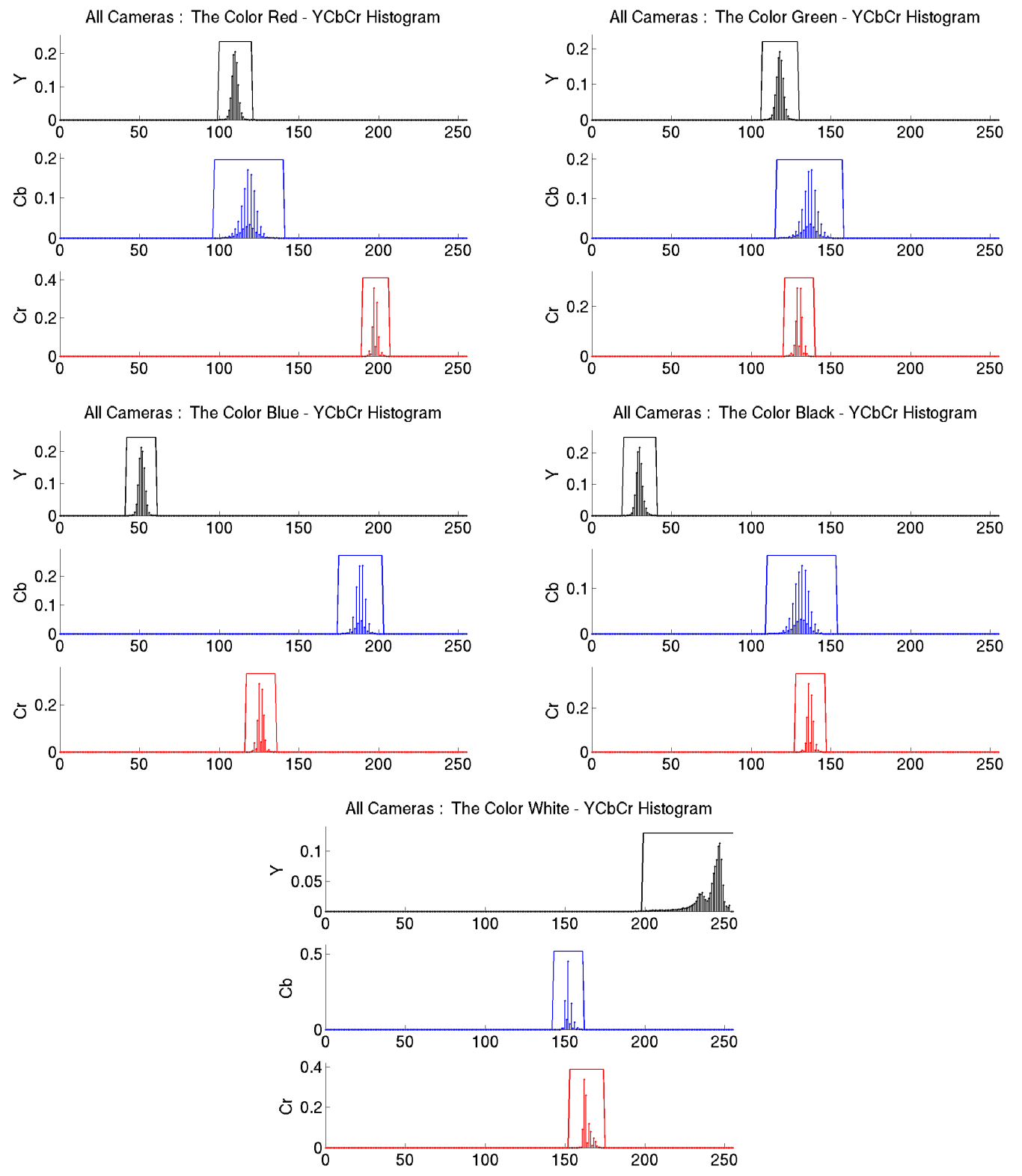

FIGURE 26-From the Left: YCbCr Histograms of Red, Green, Blue, Black and White, taken from samples of all cameras, in order to determine proper thresholding range for the colors. The bounding boxes shown on the histograms indicate good thresholding ranges. 

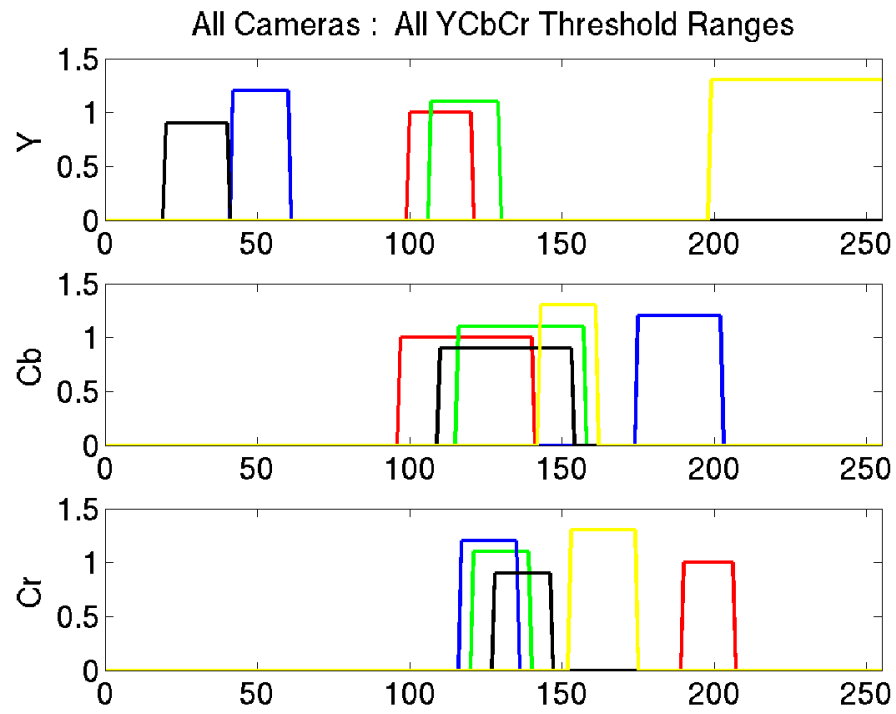

FIGURE 27 - Threshold ranges in the YCbCr space for the colors Red, Green, Blue, Black and White. White is shown as Yellow for visibility.

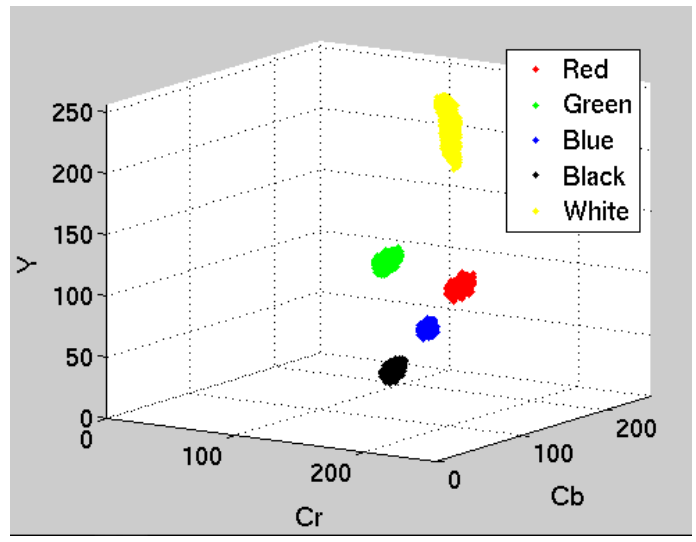

FIGURE 28 - Three dimensional plots of the colors Red, Green, Blue, Black and White in the YCbCr color space. White is shown as Yellow for visibility.

black and blue are never classified against each other. For the single robot algorithm, black is not even used. For the multi-robot algorithm, black is classified against white only. Blue is thresholded against green and white. For these two groups, the colors are linearly separable and therefore easy to classify with little error. The separability of the colors can also be seen in a three dimensional plot in Fig. 28, with each axis corresponding to the three color channels of the $\mathrm{YCbCr}$ space. Hyperplanes can easily classify these groups of 


\section{(6)}

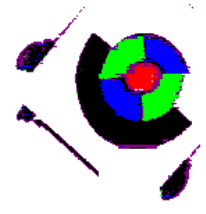

FIGURE 29-Thresholded version of Fig. 8. This was thresholded in the $\mathrm{YCbCr}$ color space and turned out very well. The purple pixels represent ones that were not classified as any one of the primary colors: Red, Green, Blue, Black or White.

colors.

Figure 29 shows a properly thresholded image from the testbed. Notice this is the same image as in Fig. 8 in Chapter 3, except now, it has been thresholded. The thresholding performed here is only done for the primary colors of red, green, blue, black and white. There is another color present in this image and that is purple. Purple is used to show that a pixels was not classified as any other color. These non-classified pixels occur frequently in this image but are expected. They appear because of shadows cast, or the occasional view of the robot beneath the hat. Another place they crop up is surrounding the primary colors. This is due to one form of discretization error.

Figure 30 shows an example of the origins of this error. On the left is a continuous image of a circle, overlaid with a sampling grid. On the right, is a gross sampling of the continuous image. Each block of the grid, or sampling square, represents a pixel in a digital image. Notice how the curves of the circle are converted to blocked edges. This is one form of discretization error. The other is in the values of the pixels. Within each sampling square, the color level is averaged. The average color in each block, or pixel, becomes the level in the discrete image. Inside the circle, or outside the circle, the color level is rendered perfectly, because the levels inside these pixel blocks are uniform. However, on the edge of the circle, the color becomes something between the background, white, and the circle, black. This is exactly what happens when a digital camera samples a continuous world.

After sampling the continuous image, the boundary of the circle is neither black, 

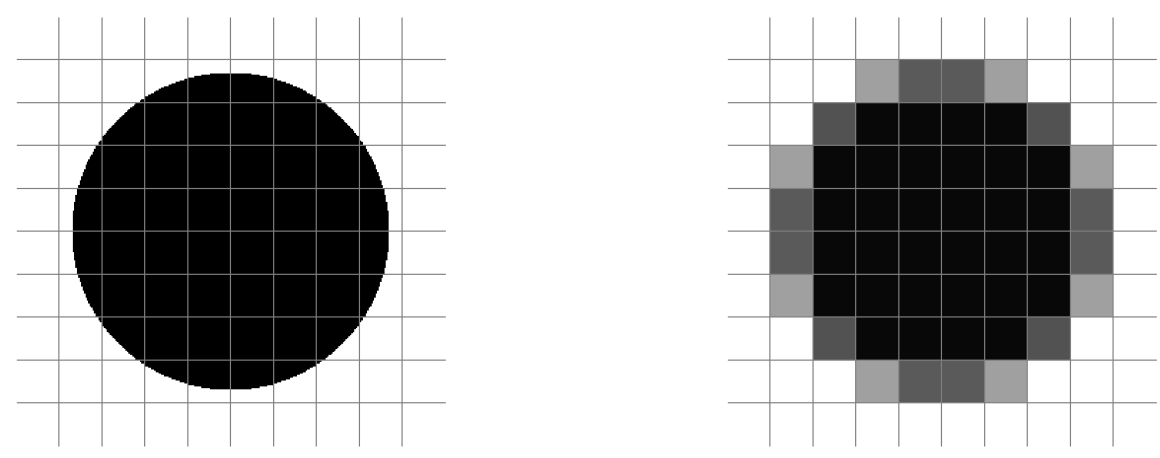

FIGURE 30-Example of discretization error caused by sampling a continuous image. The left shows the continuous image of a circle with a sampling grid over the image. The right shows the low-resolution sampling of the continuous image.

nor white. If this boundary were thresholded with the method used by the testbed, the boundary pixels would not be classified as either black or white. The same is true for the pixels in Fig. 29. When any two colors meet, there is a bleeding effect caused by sampling the image. This bleeding effect can be seen in the boundary of the circle in Fig. 30. It was expected and accounted for in the design of the hat patterns.

The small black ring surrounding the red circle in the multi-robot hat is in place to cause uniform error between all of the robot hats. Immediately surrounding the black circle is a band which is unique to every robot. Different colors meeting on edge produce different bleeding effects. Some colors could average together with red to produce a color still in the red threshold range. This would cause a shift in the center of the pattern and be a source of error for calculating the position of each robot. The black ring acts as a constant for the bleeding effect, so that every multi-robot pattern experiences the same black-red averaging, regardless of the colors present in the robot number circle.

One issue with thresholding for the testbed is the cost of misclassification. The expense of classifying a blue pixel as green is quite high. That would mean that a robot would get mislabeled as having a different robot number. As a consequence, the pose data would never get sent to the correct robot. If a pixel is not classified as any of the primary 
colors, the search will simply continue with the next available pixel. For the robot number search, there are many pixels within the quarter circle that can be tested. If the algorithm is unsure about a particular pixel, it is much better to look at another sample than to make a good guess.

For this reason, the threshold regions are purposely set small. If a pixel goes unclassified, therefore having a negative match, the algorithm still has a very high probability of success. On the other hand, expanding threshold ranges will lead to false positive matches, causing the algorithm to fail.

The most critical of all colors is red. It was chosen as the most important color because of its separability in the color space. The search algorithms perform the subsampled searches, keying on red. When a red pixel is discovered, some event is triggered, usually a detailed search. The red pixels are used to calculate the center of mass. If the threshold range is too narrow, the center of the robot could be shifted. A shift in the center of mass could cause the multi-robot algorithm to fail. On the contrary, if the threshold range is set too wide, other meaningless pixels could be mislabeled red, triggering a wasteful area search. There are stopgaps in place to recognize a false search or perform despite a shifted center; however, the balance between setting the red threshold range too large or small must be kept in check.

\section{Position Calibration}

Once the images are thoroughly thresholded and searched, the resulting position measurements are in units of pixels. In order for that information to be very useful to a robot, it needs to be converted to a unit such as meters. It is only a simple scaling factor. That scaling factor was found by measuring an object at the height of the robots in pixels and meters. The relation is a simple ratio. This was performed for both the vertical axis and horizontal axis of the camera. They were found to be only slightly different.

The origin of the testbed was selected as one corner of the room. From there, the 
$x$ and $y$ axis extend in the positive direction. Typically, the axes of an image are defined as the positive $x$ moving across the image and the positive $y$ axis moving down the image. The origin is said to be in the upper left corner of the image.

The origin is still in the same place from image to testbed, but the axes in the images have been switched. This was done to keep with the standard definition of global axes for mobile robots. Had this not been done, the orientation angle, $\theta$, would have been defined opposite of the norm, causing much confusion. 


\section{CHAPTER V \\ PERFORMANCE OF 2D TESTBED}

This chapter examines the actual performance of the testbed. The original design goals will be studied for success or failure. Some of the goals could be assessed quickly, such as the proper utilization of space. The testbed was built in a near-square shape, allowing robots to move equally in all directions. Other goals required more study to prove success, namely, speed, accuracy, support of multiple robots, and proper communication while using a generic hardware platform.

\section{A. Accuracy}

The first of the goals examined is accuracy. It is very difficult to measure the accuracy of the camera system while the robot is in motion. At the millimeter precision, it is nearly impossible to reconcile the difference between the pose calculated by the vision system and the absolute position of the robot. In order to have some idea of the accuracy of the system, several measurements are taken while the robot is not moving [1]-[7]. Figure 31 shows a scatter plot along with histograms of 10,000 position measurements taken of a stationary robot using the single robot algorithm. The measurements of the orientation angle, $\theta$, are represented in the histogram. From the figure, the algorithm performed quite well. Notice the scale of the axes are $10^{-3}$ meters. The position measurements were shifted to the origin for easier viewing. The farthest outliers are within $2 \mathrm{~mm}$ of the mean. The histogram shows that the $\theta$ measurements vary within $\pm 0.75^{\circ}$. This is an impressive feat and exceeds the original expectation of the orientation measurement.

The process was repeated for the multi-robot algorithm as well. Those results are 

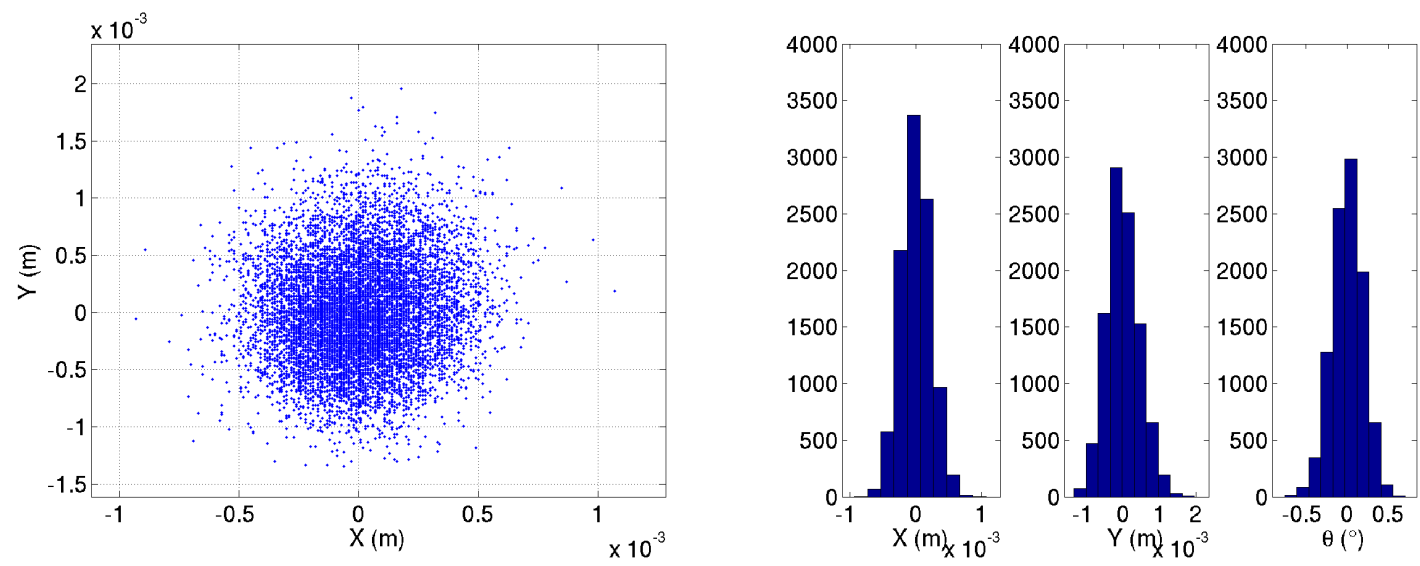

FIGURE 31 -Scatter Plot and Histogram of 10,000 measurements for a stationary robot using the single robot algorithm and hat pattern. Notice the scale of the axes is $10^{-3}$.

shown in Fig. 32. This algorithm performed almost as well. The distribution was spread slightly more, with outliers of $2.1 \mathrm{~mm}$ from the mean. The orientation angle, $\theta$, is shown in the histogram to vary $\pm 1^{\circ}$ from the mean. This variance is only slightly larger than the angle measurement from the single robot algorithm. This increase is a result of the loss of precision in the multi-robot algorithm. Remember that the single robot algorithm measures the orientation from an arctangent calculation. This gives a continuous distribution of values. The multi-robot algorithm measures $\theta$ by doing a circular search around the black/white semi-circle feature. The algorithm searches for one of the black/white edges. Therefore, this method is limited by the resolution of the image. Currently, the resolution of $\theta$ is limited to $\frac{1}{2}^{\circ}$ increments. These small variances present in both algorithms certainly meet the accuracy goals set for this testbed.

\section{B. Speed}

One major design goal for this testbed was real-time performance. The upper limit of the measurement frequency is set at $30 \mathrm{~Hz}$ by the frame rate of the overhead cameras. With the first implementation of the tracking algorithms, the measurement frequency was a sluggish $1.5 \mathrm{~Hz}$, as discussed previously. This rate was unacceptable to be used as a 

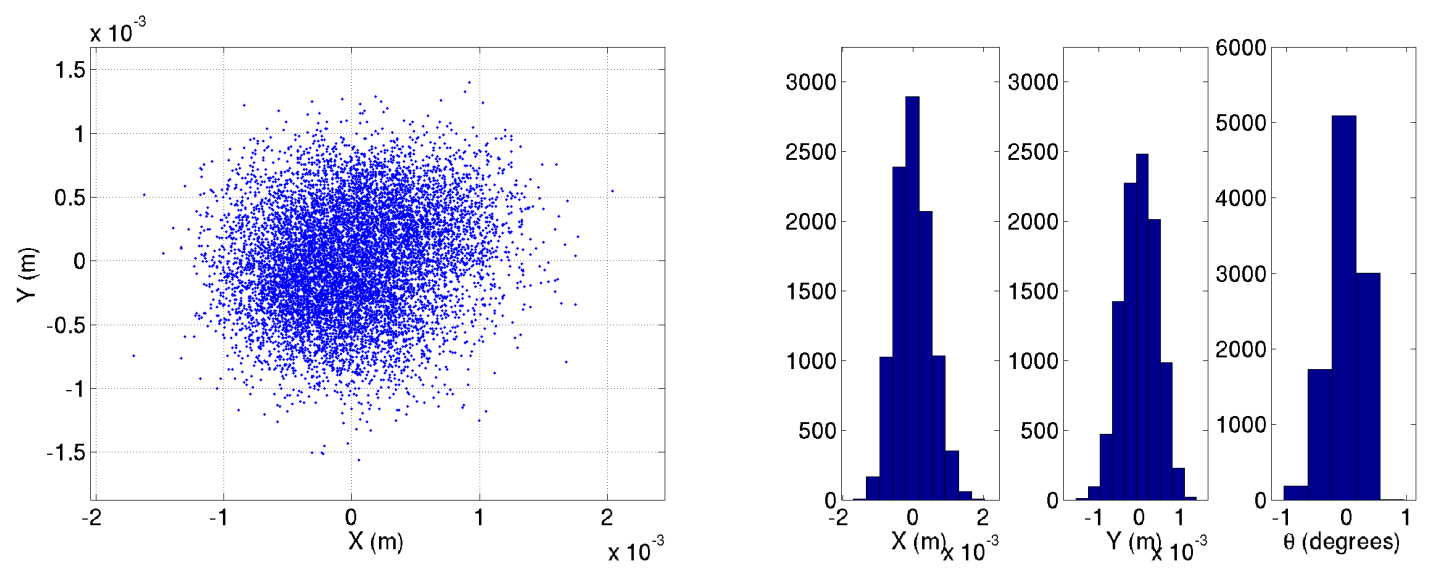

FIGURE 32 - Scatter Plot and Histogram of 10,000 measurements for a stationary robot using the multi-robot algorithm and hat pattern. Notice the scale of the axes is $10^{-3}$.

real-time system.

After a major change of drivers, the frequency spiked to $30 \mathrm{~Hz}$ for both algorithms. Ten thousand measurements were taken and the experiment was timed. In 333.3 seconds, the 10,000 position measurements were made for the single robot algorithm. The process was repeated for the multi-robot algorithm using the three robots available. Even with three robots in the testbed, the measurement frequency performed at $30 \mathrm{~Hz}$. This is certainly fast enough to meet the real-time goal for the testbed.

\section{Other Goals}

Given that the previous experiment was performed with three robots, it is apparent that the testbed is able to support multiple robots. Consequently, this design goal is satisfied as well. Also, during the experiment, the pose measurements were broadcast to the intended receivers using the UDP protocol described earlier. The pose messages were received free of error through the wireless $802.11 \mathrm{~g}$ router. This success satisfies the communications requirement of the testbed. All of these experiments were performed using a single, normal-grade, desktop computer. No specialized digital image processing hardware was required to meet the measurement frequency goal of $30 \mathrm{~Hz}$. This accomplishment sat- 

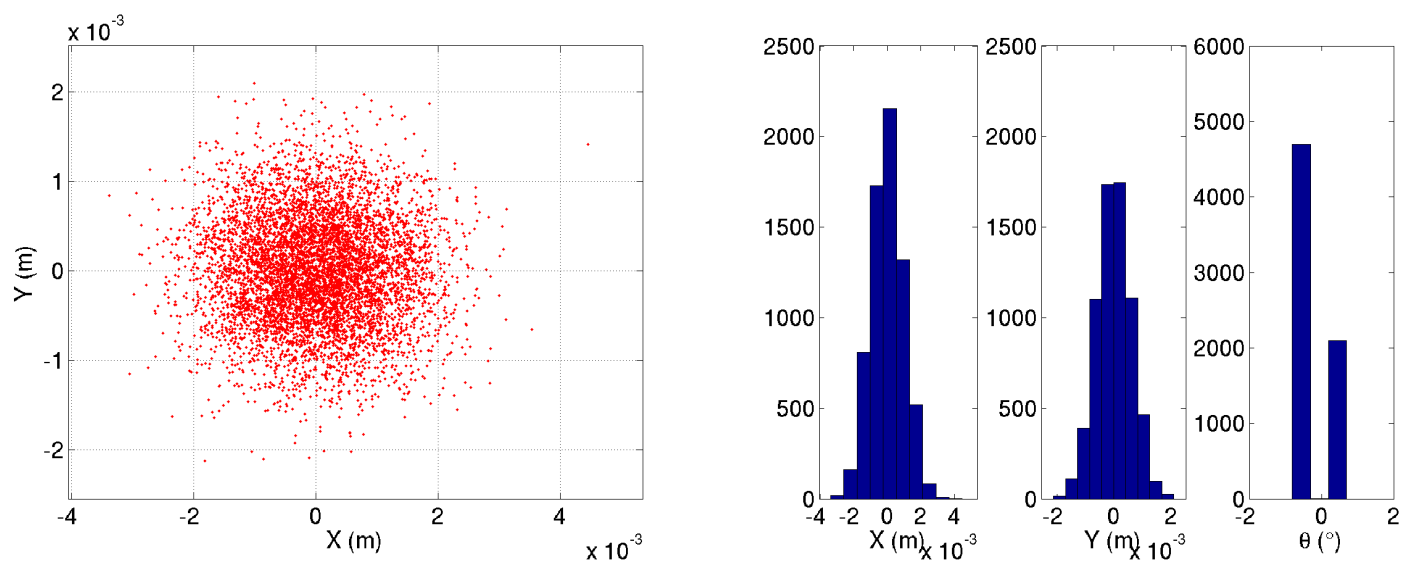

FIGURE 33 - Scatter Plot and Histogram of 10,000 measurements for a stationary robot using the multi-robot algorithm and hat pattern. To add noise to the system, some of the overhead lighting was shut off. Notice the scale of the axes is still $10^{-3}$, though the variance has increased.

isfies the design goal of using a generic hardware platform to perform robot tracking. The tracking algorithm is implemented completely in software, making it highly flexible to the changing needs of robotics research.

\section{Robustness to Noise}

As an added measure of the performance of the testbed, noise was added to the environment by changing the lighting conditions. Changes in lighting conditions are a considerable source of failure for computer vision algorithms. It is used here to the measure the robustness of the system.

After switching off several lights in the laboratory, the accuracy experiment was performed again. Figure 33 shows the results of those measurements in a scatter plot of the position, accompanied by histograms of the pose. These measurements were taken using the multi-robot algorithm. It is the more complex of the algorithms and is therefore, more likely to fail. The scale of millimeters did not change in the scatter plot, but the variance approximately doubled with the noise. Accuracies could be reported as $\pm 4 \mathrm{~mm}$ here. The orientation angle, $\theta$, experienced a little less trouble, still showing a range within $1^{\circ}$ of the mean. Although, it should be noted that the histogram of $\theta$ shows a decrease in resolution. 
The step size made an apparent increase to $1^{\circ}$ from $\frac{1}{2}^{\circ}$.

This experiment was repeated for extremely low illumination, but the algorithm failed completely. It would not acknowledge that there were any robots in the testbed. The good news is that if there is some noise present, and the algorithm is tracking robots, the error margin will be only slightly increased. If the noise is too large, it will be apparent by not tracking at all, rather than yielding even larger error margins. 


\section{CHAPTER VI MULTIPLE MOBILE ROBOTICS PLATFORM}

\section{A. Hardware}

In order to perform experiments and give position feedback in real-time, a capable hardware platform is used. The four cameras overhead are Sony UC5300 auto-focus, zoom cameras with a 1/4 inch high resolution CCD sensor [13]. The video data feeds into a Video-4-Linux MDVA3000 frame-grabber [14], which is capable of capturing eight fullrate NTSC video signals, at a resolution of $640 \times 480$, at 30 frames per second. An HP Pavilion a1430n desktop computer with an 64-bit AMD Athlon X2 dual core $1.0 \mathrm{GHz}$ processor and 2.0 G-bytes of RAM performs the image processing to track multiple robots. The wireless communications with the mobile robots is done through a standard Linksys $802.11 \mathrm{~g}$ router.

The robots themselves are Evolution Robotics ER1's [15]. The ER1 is a highly flexible unit that allows the physical structure of the robot to be designed and built by the user. The robot is made of various beams and connectors that can be used to create a variety of structures. Currently, there are three ER1's in the lab, all of which have a different shape and size. The robots are shown in Fig. 34. One robot is in the standard Evolution Robotics configuration. The second robot is near the same size, but built differently to allow easier access to the driver hardware than the standard design. The third robot is built with a hitch and trailer, allowing for some interesting movement experiments to be performed. One other advantage of this robot, besides the flexibility of the body style, is the cost. A single ER1 only costs $\$ 300$. Most mobile robotic platforms are near the $\$ 2,000$ range, and are not 


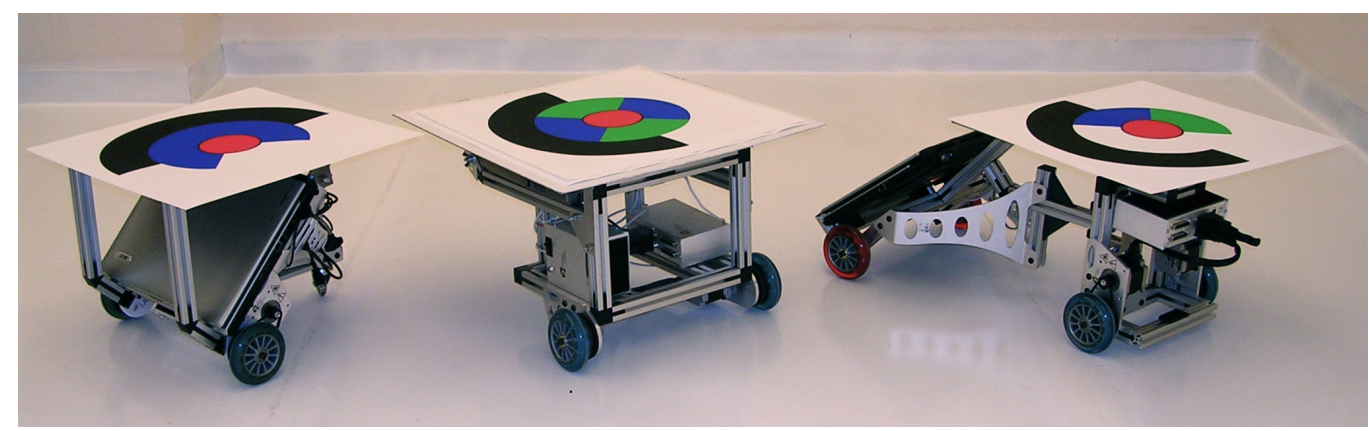

FIGURE 34 - Picture of 3 Evolution Robotics ER1's. The robot bodies can be reconfigured to build a variety of shapes. The standard configuration is on the left. The robot to the right is the hitch and trailer style. All of these robots are shown wearing their "hats", for tracking by the camera system.

nearly as flexible. The ER1 hardware is driven by a laptop computer, which interfaces via Universal Serial Bus (USB). The control box also connects to two powerful stepper motors as well as other input/output (I/O) devices such as infrared (IR) sensors and a gripper arm. There are also ports for analog inputs and digital inputs and outputs for custom sensors and devices.

The ability to control the robot with a laptop is a distinct advantage over other robots. Several popular robots are driven by embedded processors with limited processing speeds and memory availability. A laptop computer offers enhancements in both of these areas and also interfaces more easily with other devices such as cameras or range finding sensors.

The laptop computers that drive these ER1's consist of a couple of models. The first is a Dell Latitude 100L with a 32-bit $1.0 \mathrm{GHz}$ Intel Celeron processor and $512 \mathrm{Mb}$ of RAM. The other two laptops are Acer Aspire 5003WLCi's with 64-bit 1.8 GHz AMD Turion processors and $512 \mathrm{Mb}$ of RAM. All of the laptops are equipped with $802.11 \mathrm{~g}$ wireless cards to communicate with the camera system and each other.

\section{B. Software}

Though the hardware of the ER1 is fairly robust and highly flexible, the software 
accompanying the robot is not. Unfortunately, the ER1 was originally designed as an educational tool for middle school or high school students with no prior knowledge of robotics or programming. The control software consists of a graphical user interface (GUI) which allows a user to setup a chain of prior developed behaviors. The user can link together several behaviors to get an emergent behavior. This system is very easy to learn, but highly inflexible. It was not suited at all for research use in this laboratory.

Evolution sells a more advanced control software for the ER1 which provides the flexibility needed for research. However, with a price of $\$ 3,500$ per license, other suitable options were explored. An open-source project called Player/Stage was found to meet the research need.

Player/Stage [16] was developed at the University of Southern California as a robotics software platform. Player is a robot server which provides a hardware abstraction to physical robots and sensors. Drivers written for Player, allow Player to connect to many different robotic hardware platforms and sensing devices. Client programs can then be written to communicate to Player, without worrying about the physical robot. Player then communicates to the hardware, operating in the Client-Server model.

Stage is a two-dimensional robot simulator that communicates with Player. Stage can simulate hundreds of robots simultaneously. Robots can be built in Stage with a myriad of sensors and interact with user-created worlds. With the Player abstraction layer, client programs can be written and tested on simulated robots as well as actual robots. Often, the client does not know whether it is connected to Stage or a physical robot.

Currently, Player utilizes TCP socket communications. So, any language that is able to communicate via TCP can be used to write Player client programs. There are Player libraries written in C, C++, Java, and Python, as well as several others.

Player/Stage is one of the most widely used robotics platforms among researchers in the U.S. and the world. Since Player/Stage is open source, it is available free of charge. The combination of its popularity, open-source availability and language flexibility make 
Player/Stage a powerful software platform for robotics research.

Player/Stage was built for Linux or Unix-like operating systems. That required the laptops to run some Linux distribution. Mandriva 2006 was chosen after considering several others, mainly for its free cost and local knowledge base. Mandriva is an offshoot of the popular Mandrake Linux distribution. It is also the most widely used Linux distribution in the Electrical Engineering Department at UofL. That made it the best choice for use in the robotics laboratory.

One anecdote worth mentioning is that the Linux kernel needed to be modified in order to communicate with the ER1 hardware. Evolution Robotics applied for unique product and vendor ID's for their USB connection. So, in order for Linux to recognize the hardware, those ID's needed to be added to the current list in the kernel. These changes are listed in Appendix VI. 


\section{CHAPTER VII APPLICATIONS}

Equipped with a tool to quantify movements of mobile robots, research can be performed in many areas. There are many applications that can be studied. Anything from coordinated movement, to path planning, to obstacle avoidance can be examined with this testbed. To begin, some control experiments were conducted.

\section{A. Smooth Path Control}

First, a controller was developed to move a differential drive robot along a smooth path from any point $A$ to point $B$. A differential drive robot is one that uses two drive wheels, controlled independently by two motors. The wheel planes are parallel with each other. This constrains the movement of the side, or local $y$, direction to zero. In mobile robotics, this is known as a non-holonomic constraint. This is why differential drive robots are also referred to as a type of non-holonomic robot. Its motion is restricted to the $X_{R}$ direction, as shown in Fig. 35. Holonomic robots are free to move in any direction at any given time. For this reason, they are typically called omnidirectional robots. For an omnidirectional robot, the task of moving from point $A$ to $B$ is very simple. Without any constraints, these robots simply move in a straight line directly to the destination. The orientation can even be changed in motion without affecting the path. A differential drive robot presents more of a challenge. The kinematic constraints introduced prevent the robot from moving directly toward the goal.

One way of moving from point to point for the differential drive robot, is to simply rotate toward the goal, move in a straight path to the goal and then rotate to the desired 


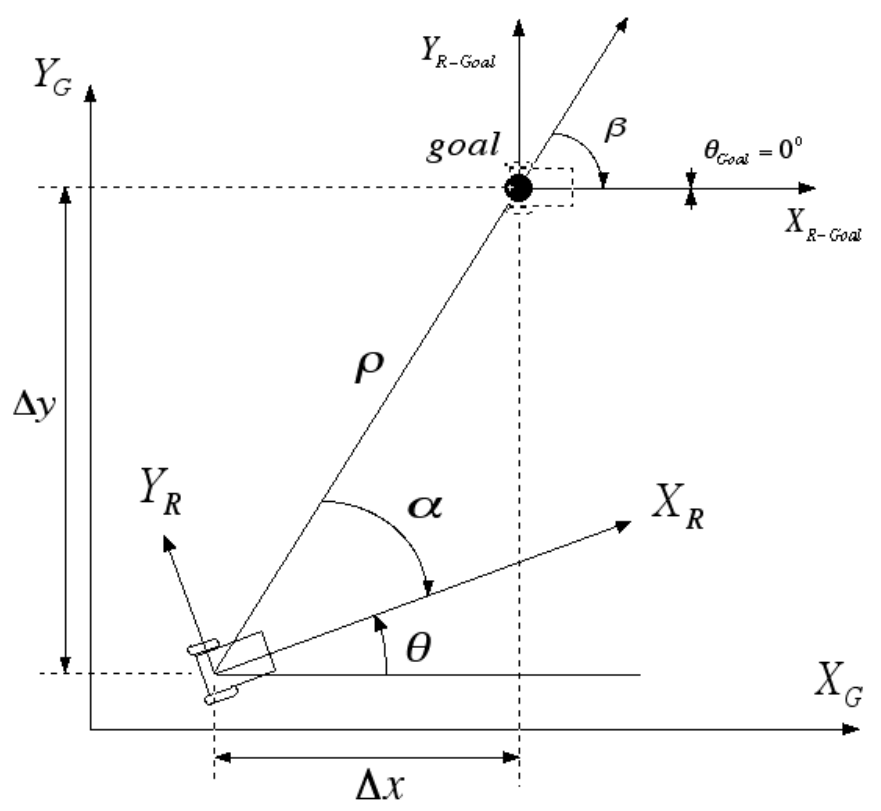

FIGURE 35-Diagram of the robot's frames of interest. The control variables $\rho, \alpha$, and $\beta$ are illustrated. The goal here is shown in the upper-right, with the orientation aligned with the global axes.

orientation. However, this approach yields discontinuities in the actual movement. This may be acceptable for small ground vehicles, but the ultimate goal of this research is to later apply these methods to actual systems. Physical systems such as automobiles or airplanes have even more constraints. Automobiles cannot rotate in place like the differential drive robot. Airplanes require minimum velocities in order to provide lift and minimum radii to turn. Most cannot hover in place.

A better approach is to find a way to control a smooth path to a specified target. This can be expressed as finding control of $v(t)$, the linear velocity, and $\omega(t)$, the angular velocity, so that the error in current position and desired position is driven to zero.

$$
\left[\begin{array}{c}
v(t) \\
\omega(t)
\end{array}\right]=K \cdot e=K\left[\begin{array}{l}
x \\
y \\
\theta
\end{array}\right]_{\text {Global Error }}
$$

The kinematics of a differential drive robot can be described by Eqn. 8, where $\dot{x}$ and $\dot{y}$ are 
the linear velocities in the global reference frame.

$$
\left[\begin{array}{c}
\dot{x} \\
\dot{y} \\
\dot{\theta}
\end{array}\right]_{G}=\left[\begin{array}{cc}
\cos \theta & 0 \\
\sin \theta & 0 \\
0 & 1
\end{array}\right]\left[\begin{array}{l}
v \\
\omega
\end{array}\right]
$$

Next, consider the coordinate transformation into polar coordinates. $\Delta x$ and $\Delta y$ are shown in Fig. 35.

$$
\begin{gathered}
\rho=\sqrt{\Delta x^{2}+\Delta y^{2}} \\
\alpha=-\theta+\operatorname{atan} 2(\Delta y, \Delta x) \\
\beta=-\theta-\alpha+\theta_{\text {Goal }}
\end{gathered}
$$

Note that atan 2 is the four quadrant inverse tangent. This new coordinate system is shown in Fig. 35. Using the new coordinate system described by (9)-(11), Eqn. 8 is transformed to give a new description of the system.

$$
\left[\begin{array}{c}
\dot{\rho} \\
\dot{\alpha} \\
\dot{\beta}
\end{array}\right]=\left[\begin{array}{cc}
-\cos \alpha & 0 \\
\frac{\sin \theta}{\rho} & -1 \\
-\frac{\sin \alpha}{\rho} & 0
\end{array}\right]\left[\begin{array}{l}
v \\
\omega
\end{array}\right]
$$

Starting at some arbitrary location, $\left(\rho_{0}, \alpha_{0}, \beta_{0}\right)$, the task becomes driving the error between the starting and final location to zero. At a minimum, this can be achieved using proportional control of the three variables: $\rho, \alpha$, and $\beta$

$$
\begin{gathered}
v=k_{\rho} \rho \\
\omega=k_{\alpha} \alpha+k_{\beta} \beta
\end{gathered}
$$

where $k_{\rho}, k_{\alpha}$ and $k_{\beta}$ are the proportional control constants for $\rho, \alpha$ and $\beta$, respectively. Substituting the control law of (13) and (14) into Eqn. 12, the system is then described by

$$
\left[\begin{array}{c}
\dot{\rho} \\
\dot{\alpha} \\
\dot{\beta}
\end{array}\right]=\left[\begin{array}{c}
-k_{\rho} \rho \cos \alpha \\
k_{\rho} \sin \alpha-k_{\alpha} \alpha-k_{\beta} \beta \\
-k_{\rho} \sin \alpha
\end{array}\right]
$$




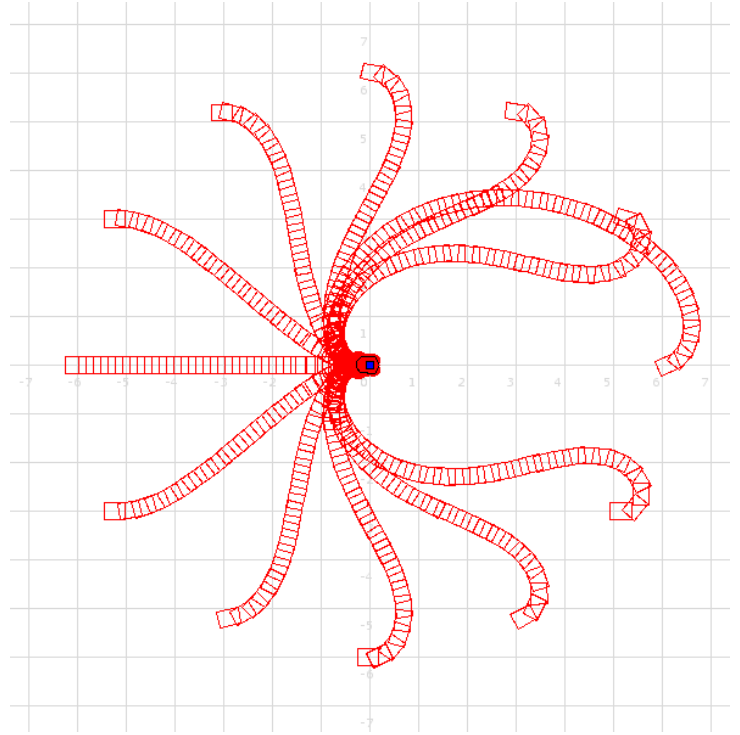

FIGURE 36-Stage simulation of the smooth trajectory controller. The robot was placed at different poses on a $6 \mathrm{~m}$ radius circle. The starting orientation angle was always $0^{\circ}$. The robot found a smooth path to the origin each time.

More details for this derivation can be found in [17]. This system will drive the robot along a smooth trajectory to the goal pose. It should be noted that the sign of $v$ is constant. Therefore, the direction the linear velocity of the robot is restricted to either forward or backward. Forward was chosen for the design of this controller. This decision affects another area of the controller. The angles $\alpha$ and $\beta$ are defined using the vector $\vec{\rho}$. As the robot approached the goal, $\vec{\rho}$ gets smaller and smaller in magnitude. If the robot passes the intended goal, this vector remains small in magnitude, but suddenly changes almost $180^{\circ}$ in direction. This causes the small errors in $\alpha$ and $\beta$ to spike to almost $180^{\circ}$ as well. These large errors then provide a large input to the angular velocity. In other words, since the linear velocity is constrained to forward motion, the robot attempts to circle around and make another pass at the goal pose. This situation is corrected by monitoring the derivatives of the angles, $\dot{\alpha}$ and $\dot{\beta}$. Whenever the derivatives spike in value, the controller is stopped.

The experiment was first performed in the simulator Stage, as seen in Fig. 36. The robot was placed at different locations around a $6 \mathrm{~m}$ radius circle and commanded to move to the origin. The orientation at each starting location was $0^{\circ}$. The controller was then tested 


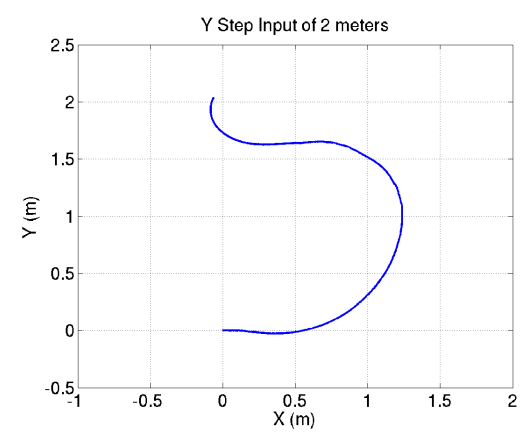

FIGURE 37 - Stage simulation and actual experiment of the smooth trajectory controller. The command was to move from the $\left(0,0,0^{\circ}\right)$ to $\left(0,2,0^{\circ}\right)$. The robot moved along a smooth path in the simulator and the hardware. The hardware shows a small steady-state error though.

with the ER1 robot. Figure 37 shows the path taken by the ER1 when given a command to move from $\left(0 \mathrm{~m}, 0 \mathrm{~m}, 0^{\circ}\right)$ to $\left(0 \mathrm{~m}, 2 \mathrm{~m}, 0^{\circ}\right)$. This figure shows the results in the simulator and the hardware. Notice that there is a small steady state error in the final position with the ER1. It is infeasible to reach any given position exactly. There is a limit to the accuracy of the position measurement. There is also a small time delay between measurements and movements. The actual controller is implemented using a threshold within a given error margin to decide when the goal pose has been reached. When the robot arrives within the acceptable threshold range, the controller stops.

\section{B. Circular Path Control}

The second application was the control of a differential drive robot along a circular path. All of the hardware experiments were performed in Stage first. Stage serves as a useful tool to quickly prototype an algorithm without the potential of damaging physical robot hardware. There are also very few changes that need to be made to the program when moving from the simulator to actual hardware. 


\section{Simulation}

In the initial controller design, the radius of the circle was tracked. A constant linear velocity was maintained, while the error between the desired radius and the current radius proportionally controlled the angular velocity of the vehicle. The results of this method are shown in Figure 38. These images are taken from Stage at different intervals of the simulation. The robot is shown as the solid red polygon. The red trails show the path taken by the robot for illustration. In this simulation, the robot attempts to track a circle of radius $3 \mathrm{~m}$ in the counter-clockwise direction with a constant linear velocity of $0.2 \mathrm{~m} / \mathrm{s}$. The robot is given a starting location directly on the desired radius. As Fig. 38 shows, this controller is unstable. The oscillations start to occur during the first pass of the circle. They grow in the second pass. The robot does not even finish the third trip around the circle. The oscillations grow so much, that the robot actually moves in the opposite intended direction as shown in the last frame.

This experiment was performed for several different proportional gains, but the results were the same. Simply changing the proportional gains did not fix this problem. For the non-holonomic robot, this controller design was inherently unstable. Some other control variable was needed. As several others have done, the tangent angle was added to the feedback loop [18], [19]. Now, the robot not only tracked the radius of the circle, but also the angle tangent to the circle at the robot's position. Equation 17 gives the new control law, where $\rho$ is the radius error and $\tau$ is the error between the current orientation and the tangent of the circle. This brought stability to the system.

$$
\begin{gathered}
\tau=\theta-\theta_{\text {tangent }} \\
\omega=k_{\rho} \rho-k_{\tau} \tau
\end{gathered}
$$

Figure 39 shows the results of the experiment with the added angular control. The experiment was the same, with the exception of the starting location. This controller performed so well, that robot could be positioned at different poses and successfully track the circle. 


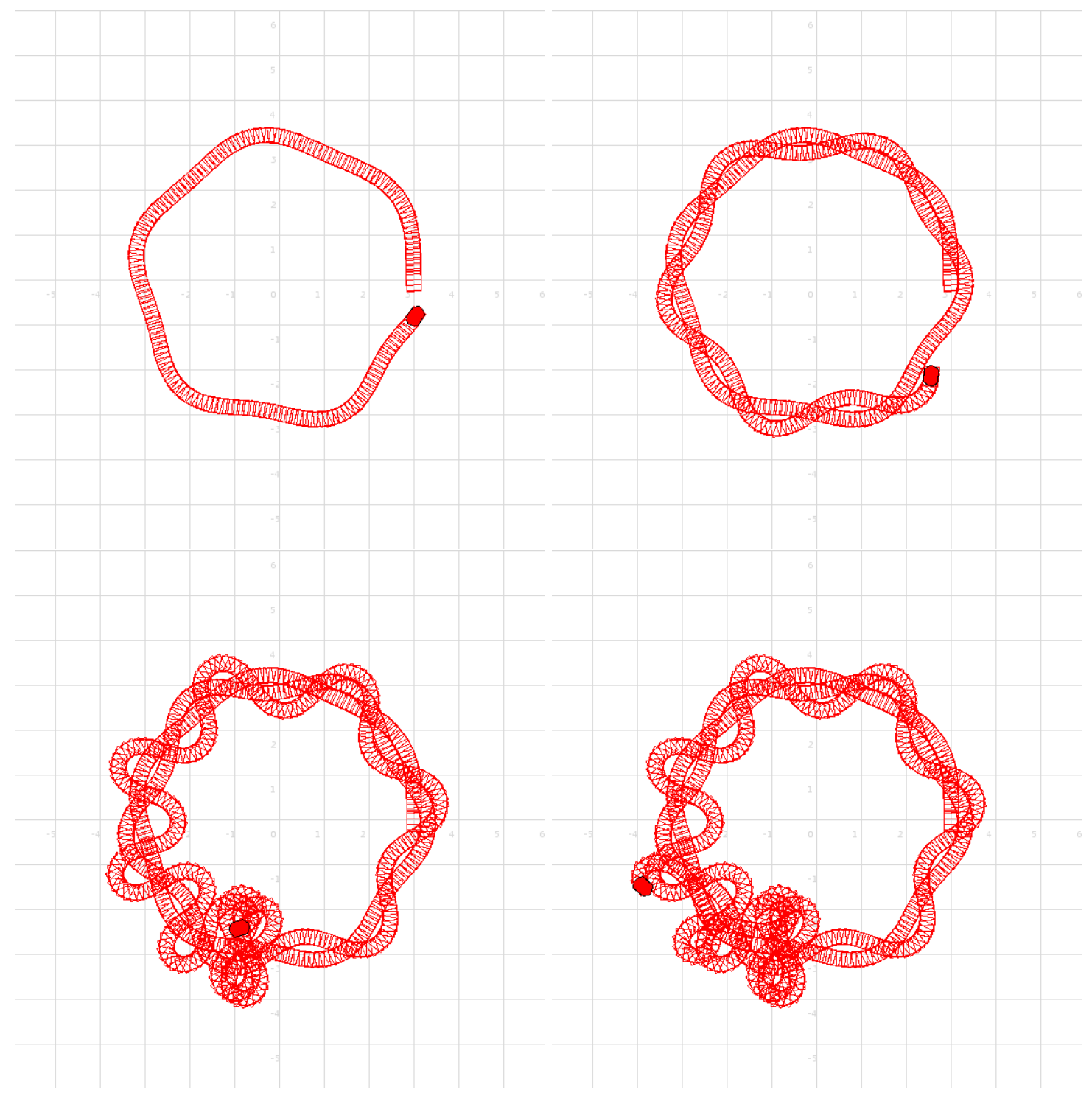

FIGURE 38 - Simulation of circular path controller, tracking the radius at 3 meters, with a forward speed of $0.2 \mathrm{~m} / \mathrm{s}$. The starting point was $\left(3.0,0,90^{\circ}\right)$. As time progressed, the oscillations grew until the algorithm failed. 


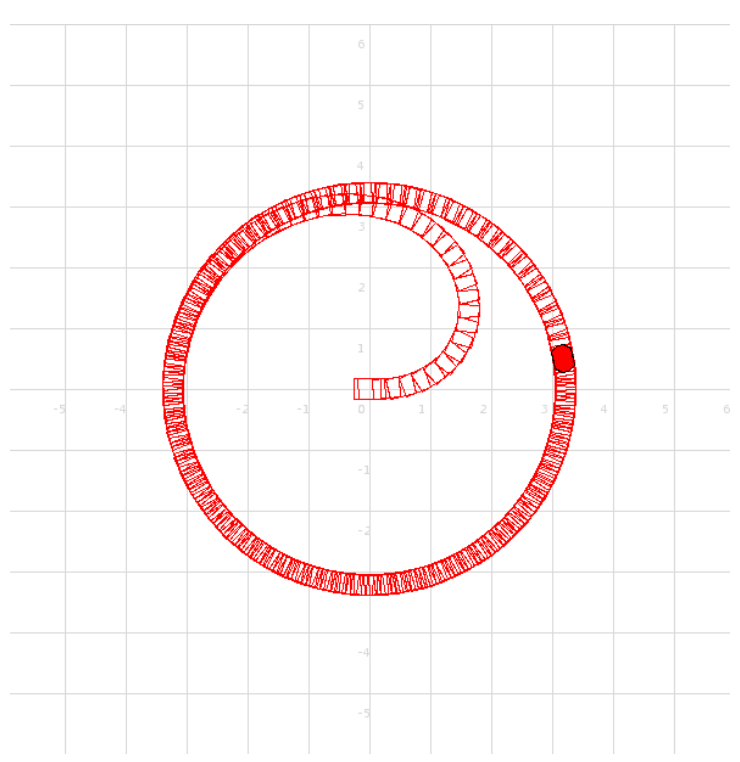

FIGURE 39-Simulation of circular path controller, tracking the radius of 3 meters and the tangent angle of the circle, with a forward speed of $0.2 \mathrm{~m} / \mathrm{s}$. Even when the starting location was the origin, this controller performed very well.

Here it was started at the origin. This frame was taken after three passes around the circle. One thing to note in Fig. 39 is the steady state error present in this controller. The center of the robot should pass directly over the $3 \mathrm{~m}$ radius. In the figure, the inside wheel of the robot is actually just outside the $3 \mathrm{~m}$ radius. This could be corrected by adding integral control to the feedback loop.

\section{Single Robot: Odometry and Camera System Feedback}

Once the control algorithms performed well in the simulator, they were applied with the physical robots in the testbed. In the simulator, there is only one type of pose feedback. It consists of the "Eye of God" perfect measurement. With the robot hardware, there is not the benefit of perfect measurement.

The ER1's are equipped with internal odometry to keep track of their pose. With the testbed, there is also the possibility of receiving feedback through the vision system. Both of these systems were examined during these experiments. 


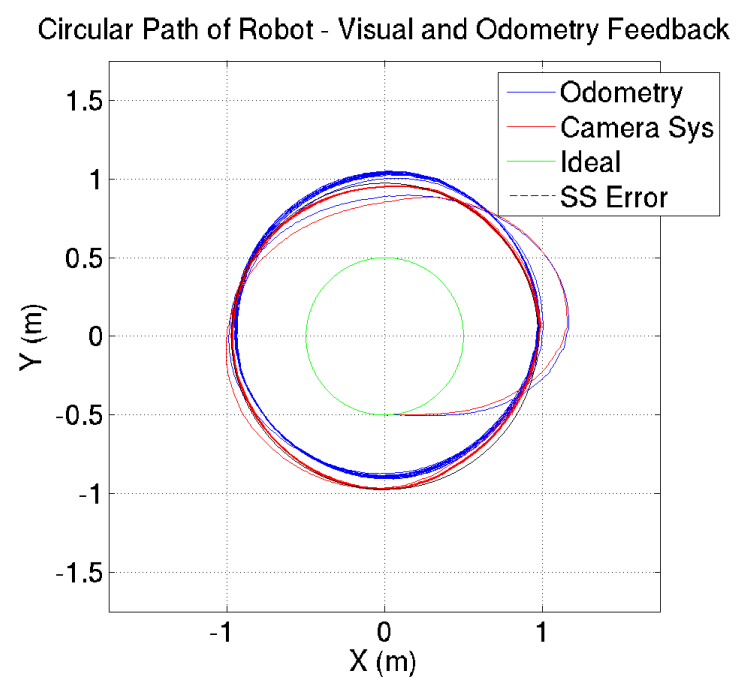

FIGURE 40 - Experiment of circular path controller in testbed. Tracking is done for a 0.5 radius and forward speed of $0.2 \mathrm{~m} / \mathrm{s}$. Plot shows the experiment with odometry feedback in blue, and camera system feedback in red. There is a steady-state error present in this controller.

Figure 40 shows the results of the hardware experiments. The robot was given a circle of radius $0.5 \mathrm{~m}$ to track with a constant linear speed of $0.2 \mathrm{~m} / \mathrm{s}$. For these experiments, the robot started directly on the circle to track. The figure shows the results of the experiment using both the internal odometry as feedback and the overhead camera system as feedback. The steady state error mentioned previously is more apparent here than in the simulations. The error draws to half a meter.

The vision system proves to be more accurate than the odometers. There is an apparent shift in the odometer's sense of the origin as it makes its first pass around the circle. This is a well-documented error found in odometers. The point of origin suffers from a "drift" as the robot moves. After the shift, the odometers are fairly consistent, but there is a larger variance as the robot continues to track the circle. This can be seen from the spread of the blue lines in various sections of the path. As the robot moves, the odometry uncertainty grows. The variance in the vision system is much smaller. Of course, it keeps track of the origin as well.

Since the orientation angle, $\theta$, was also measured during the tracking experiment, that information was plotted in Fig. 41. This is a representation of the movements of the 


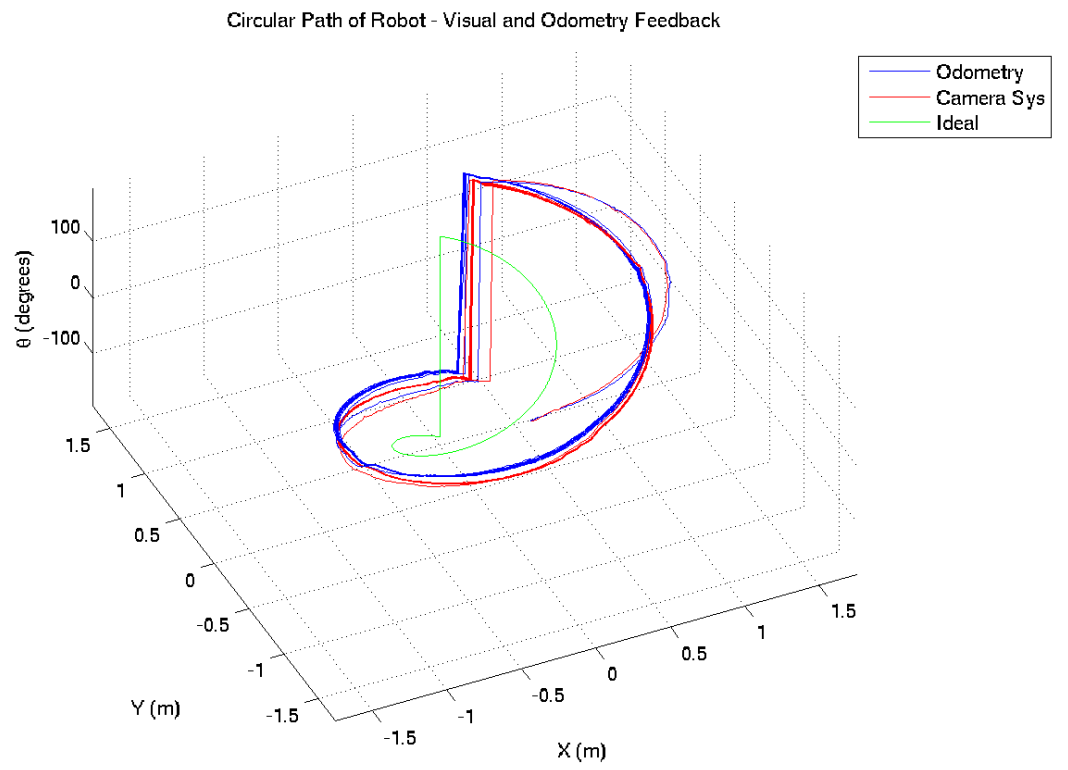

FIGURE 41 - Experiment of circular path controller in testbed. Tracking is done for a 0.5 radius and forward speed of $0.2 \mathrm{~m} / \mathrm{s}$. Plot shows the experiment with odometry feedback in blue, and camera system feedback in red in three dimensions to view the change in $\theta$. The discontinuity is expected as $\theta$ moves from $180^{\circ}$ to $-180^{\circ}$.

robot in three dimensions, with the vertical axis representing $\theta$. The sharp discontinuity is expected as the robot moves around the circle from $180^{\circ}$ to $-180^{\circ}$.

One interesting feature present in this diagram, not represented in the previous, is the consistent "ripples" in the path. These are very small but repeated shifts in $\theta$. These can be explained by the seams in the testbed. As the robot hits the seams of the floor, there are small bumps that cause the robot to change orientation slightly. Unfortunately, the seams could not be constructed perfectly, but this effect is another testimony of the accuracy of the overhead camera system. 

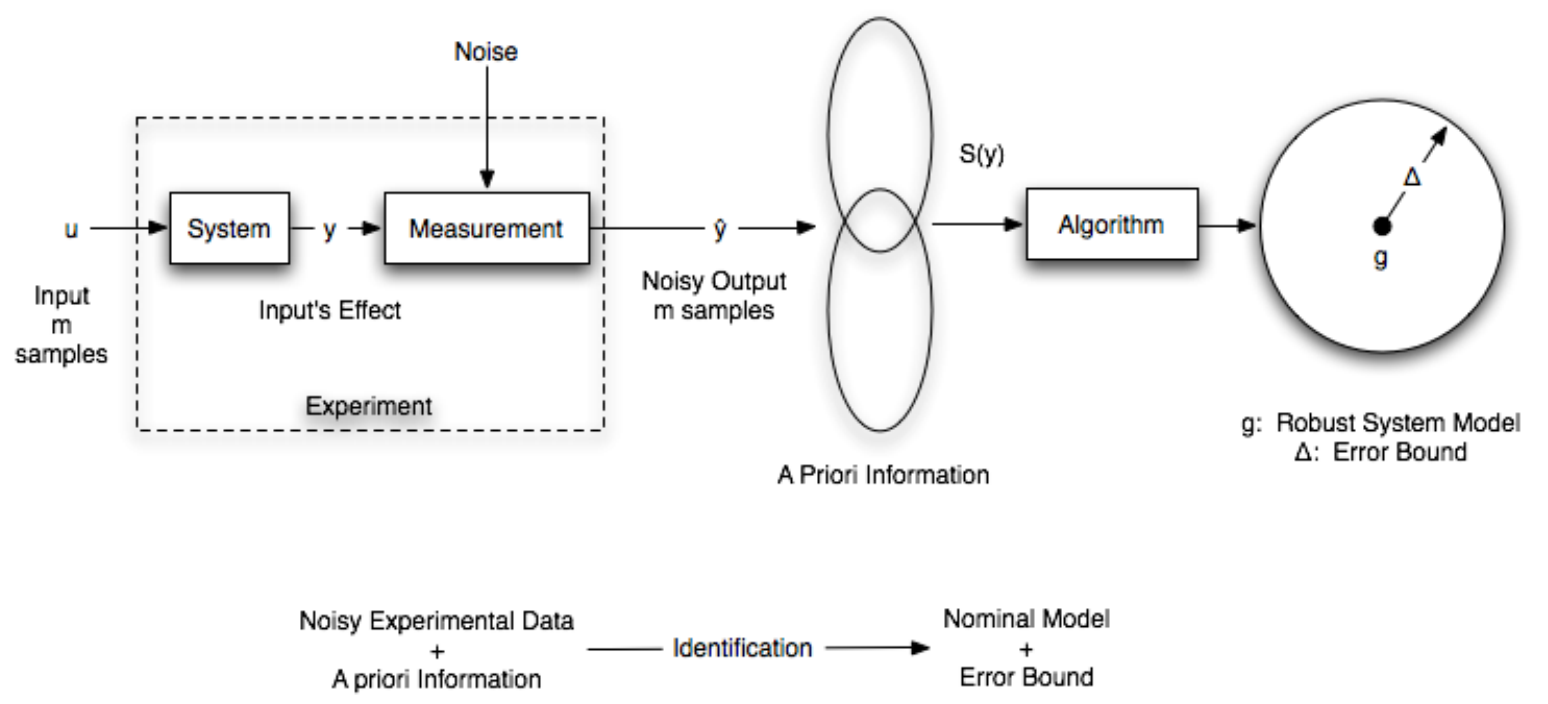

FIGURE 42 - A summary of the Robust Identification Framework.

\section{Robust Identification of a Single Robot}

Given noisy experimental data and some a priori information about the class of models (not the order of the model) robust identification procedures generate a nominal model and bounds on the identification error as shown in Fig. 42. The available a priori information consists of a lower bound on the relative stability margin of the plant, an upper bound on a certain gain associated with the plant, and an upper bound on the noise level. More detail on robust identification can be found in [20], [21], and [22]. One of the major differences and advantages of the robust identification algorithm compared to other classical modeling algorithms [21] such as maximum likelihood estimation, least squares estimation, etc., is that it is deterministic rather than stochastic. Another advantage is that it takes into account the measurement noise, and finally, it does not assume a pre-fixed mathematical structure as other classical methods do. 


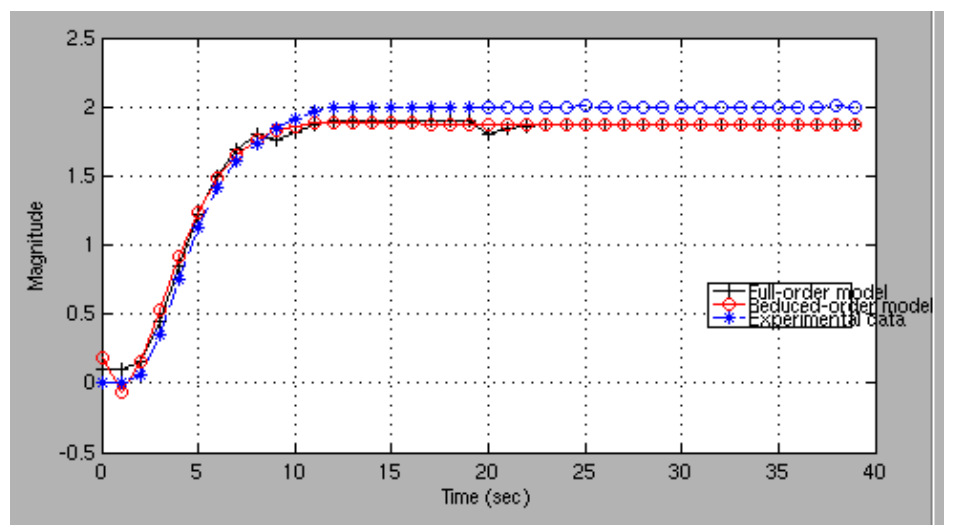

FIGURE 43 - Result of robust identification using a 2 meter step input. The identification was done using the internal odometers as feedback. The full order and reduced order models are shown in black and red, respectively. The actual output is shown in blue. The reduced order model was reduced from $25^{\text {th }}$ order model to a $2^{\text {nd }}$ order model.

\section{Modeling with Odometry Feedback}

In order to model one of the ER1 robots, a step input was supplied to the robot. The step input was given as a change in position of 2 meters in the forward direction. The resulting output was then measured. Both the output and input were supplied to the robust identification algorithm. This process was performed twice. The first model generated was done using the robot's internal odometers as position feedback. The second experiment was done using the overhead vision system. Figure 43 shows the result of the time domain experiment using the odometers as feedback. The blue points are the experimental data. The black and red data points show the full order and reduced order models generated from the robust identification. In this figure, “*” denotes experimental data points used in the identification algorithm, while " $\circ$ " indicates the points not used in the identification algorithm. The step response of the model matches the experimental time-domain data very well and is within the maximum error bound.

To find the upper error bound of the system, the position of the robot was measured by changing the lighting conditions. This added noise caused variation in the testbed's measurement of the robots. The maximum error at the time was measured at 0.1 meters.

These models were found using $\ell_{1}$ identification, which is strictly based upon exper- 


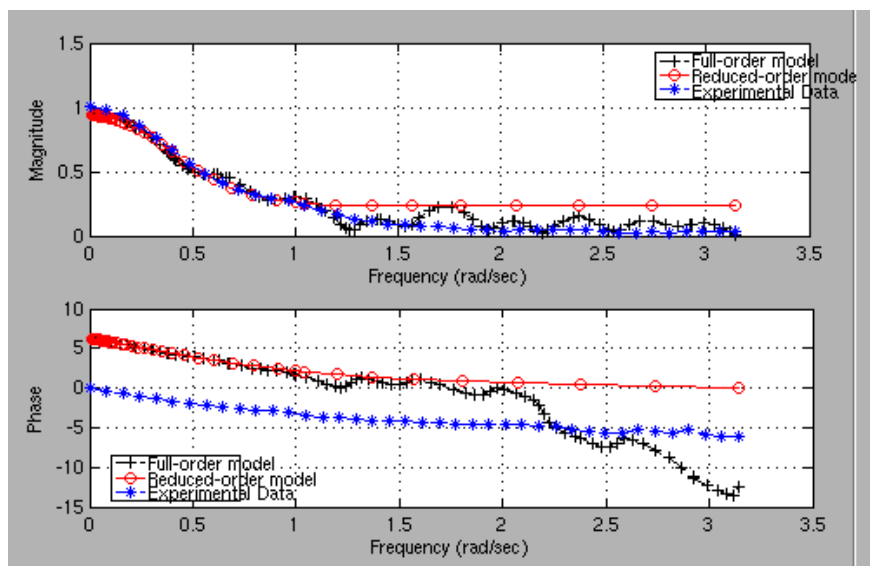

FIGURE 44 - Robust model comparison in the frequency domain. The frequency data is shown for the actual data, the full order model and the reduced model. The model matches the data well in the time and frequency domains.

imental time domain data. Another form of robust identification, known as $H_{\infty}$ identification uses frequency domain experimental data to calculate a model. There is another hybrid method, mixed $\ell_{1} / H_{\infty}$ identification which utilizes time domain data as well as frequency domain data. Figure 44 shows the frequency response of the experimental data as well as the responses of the full and reduced order models for comparison. This figure shows that the model found matches well in the frequency domain. Thus, $H_{\infty}$ or mixed identification is not needed. The model produced by $\ell_{1}$ identification is a discrete model represented in the state space. The typical system is usually defined as:

$$
\begin{aligned}
x[n+1] & =\mathbf{A} x[n]+\mathbf{B} u[n] \\
y[n] & =\mathbf{C} x[n]+\mathbf{D} u[n]
\end{aligned}
$$

The robust identification yielded the following matrices for the reduced, second order system:

$$
\begin{aligned}
& \mathbf{A}=\left[\begin{array}{cc}
0.8591 & -0.1770 \\
1 & 0
\end{array}\right] \mathbf{B}=\left[\begin{array}{l}
1 \\
0
\end{array}\right] \\
& \mathbf{C}=\left[\begin{array}{ll}
0.0101 & 0.2428
\end{array}\right] \mathbf{D}=[0.0548]
\end{aligned}
$$




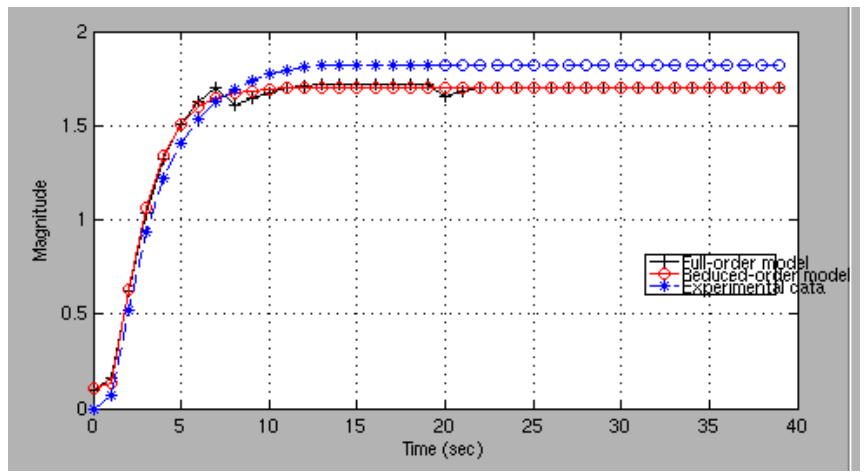

FIGURE 45 - Result of robust identification using a 2 meter step input. The modeling was done using the camera system as feedback. The full order and reduced order models are shown in black and red, respectively. The actual output is shown in blue. The reduced order model was reduced from $25^{\text {th }}$ order model to a $2^{\text {nd }}$ order model.

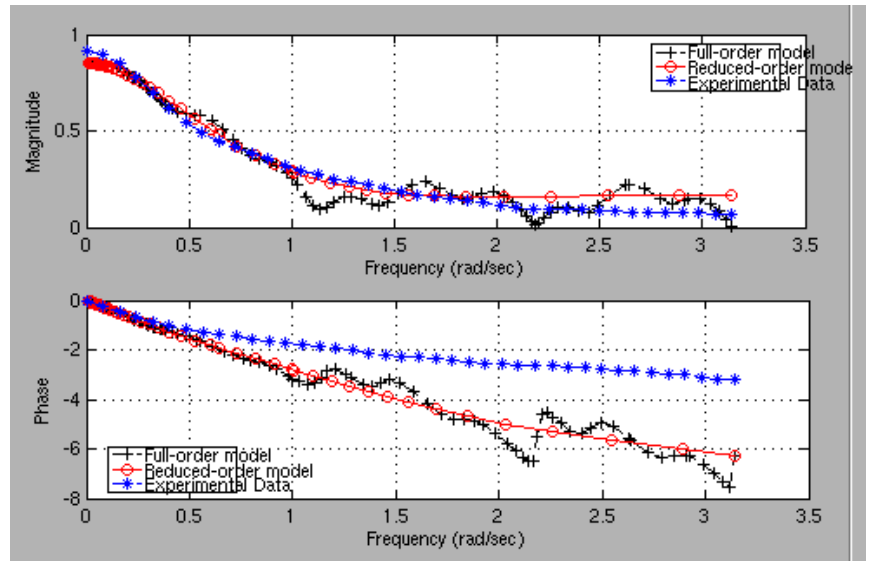

FIGURE 46 - Robust model comparison in the frequency domain. The frequency data is shown for the actual data, the full order model and the reduced model. The model matches the data well in the time and frequency domains.

The same experiment was performed using the testbed overhead vision system for the position feedback during the step input experiment. Figures 45 and 46 show the results of the $\ell_{1}$ identification. The models match well in both the time domain as well as the frequency domain. The matrices to define the reduced, second order model are given in Equations 22 and 23.

$$
\begin{gathered}
\mathbf{A}=\left[\begin{array}{cc}
1.2637 & -0.4291 \\
1 & 0
\end{array}\right] \mathbf{B}=\left[\begin{array}{l}
1 \\
0
\end{array}\right] \\
\mathbf{C}=\left[\begin{array}{ll}
-0.1240 & 0.2635
\end{array}\right] \quad \mathbf{D}=[0.0925]
\end{gathered}
$$




\section{Leader-Follow Experiments}

Currently, considerable research is being done with mobile robot coordination. These studies cover areas such as communication, distributed sensing, and environment mapping, to name a few. Another area of this research is group movement or formation movement. The formation studied here is a leader-follow formation.

To breech this topic, the smooth path controller was extended to accept a constantly varying goal state. A leader communicates its location to the follower. The follower receives the leader's position and sets the goal state at a specified distance behind the leader. The error between the follower's current position and newly defined goal state is fed to the smooth path controller. This entire process repeats as the leader moves. The leader continues to send its pose to the follower. The follower updates the goal based on the new location of the leader and calculates a new error. Both the leader and follower receive their own pose information from the overhead vision system of the testbed.

It is easy for a follower to track a leader if the follower knows the final goal of the leader. The problem of following a leader is then reduced to the follower finding its own way to the goal. This is not really following at all, but merely two independent robots moving to some final state. In the method presented here, the follower robot is unaware of the destination of the leader. The follower's task is to simply trail the leader based on current information from the leader robot.

Another thing to note is that the leader-follow algorithms use decentralized control. The robots communicate with each other to exchange pose information, but the movement control of each robot is performed by each robot. There is not a central controller that gives movement commands to the robots in the testbed. Decentralized control presents a greater challenge for the movement of multiple mobile robots. 


\section{Simulation}

The algorithm was first tested in Stage. Figure 47 shows a simulation where the leader is given the command to track a circle of radius 3 meters. The follower is not aware of the leader's intentions. It only receives pose information updates from the leader. The follower is tracking a goal state of $1 \mathrm{~m}$ directly behind the leader. The leader is shown in red, while the follower is in green. Notice that the follower moves in a slightly larger circle than the leader. This can be explained by the follower tracking a point $1 \mathrm{~m}$ directly behind the leader. Since the leader is constantly in a turn, the tracking point for the follower is cast out to a larger radius circle.

Other than the small tracking error in the circle, the follower performs remarkably well for such a simple algorithm. To test this method further, two different step inputs were given to the leader. In Fig. 48 , a goal position of $\left(2 \mathrm{~m}, 3.5 \mathrm{~m}, 0^{\circ}\right)$ was given to the leader. Once the leader arrived at that location, a second pose of $\left(5 \mathrm{~m}, 1.5 \mathrm{~m},-90^{\circ}\right)$ was given. While the leader was moving to these goal locations, it continuously sent its current pose to the follower.

Again, the follower performed very well. One thing to note, however, is that the follower tends to amplify the path of the leader. For the first step input, the leader moved within a single meter in the $x$ direction. The follower required a slightly wider path. This is actually the same phenomenon observed in the first experiment, where the follower moved in a larger circle than the leader.

\section{Experiment}

Once the algorithm proved successful in the simulator, the experiment was performed in the testbed. The leader was given a circle to track. The initial poses of the two robots were just outside the circle, with the follower approximately 0.5 meters behind the leader. For spatial reasons, the tracking distance of the follower was decreased to 0.5 me- 


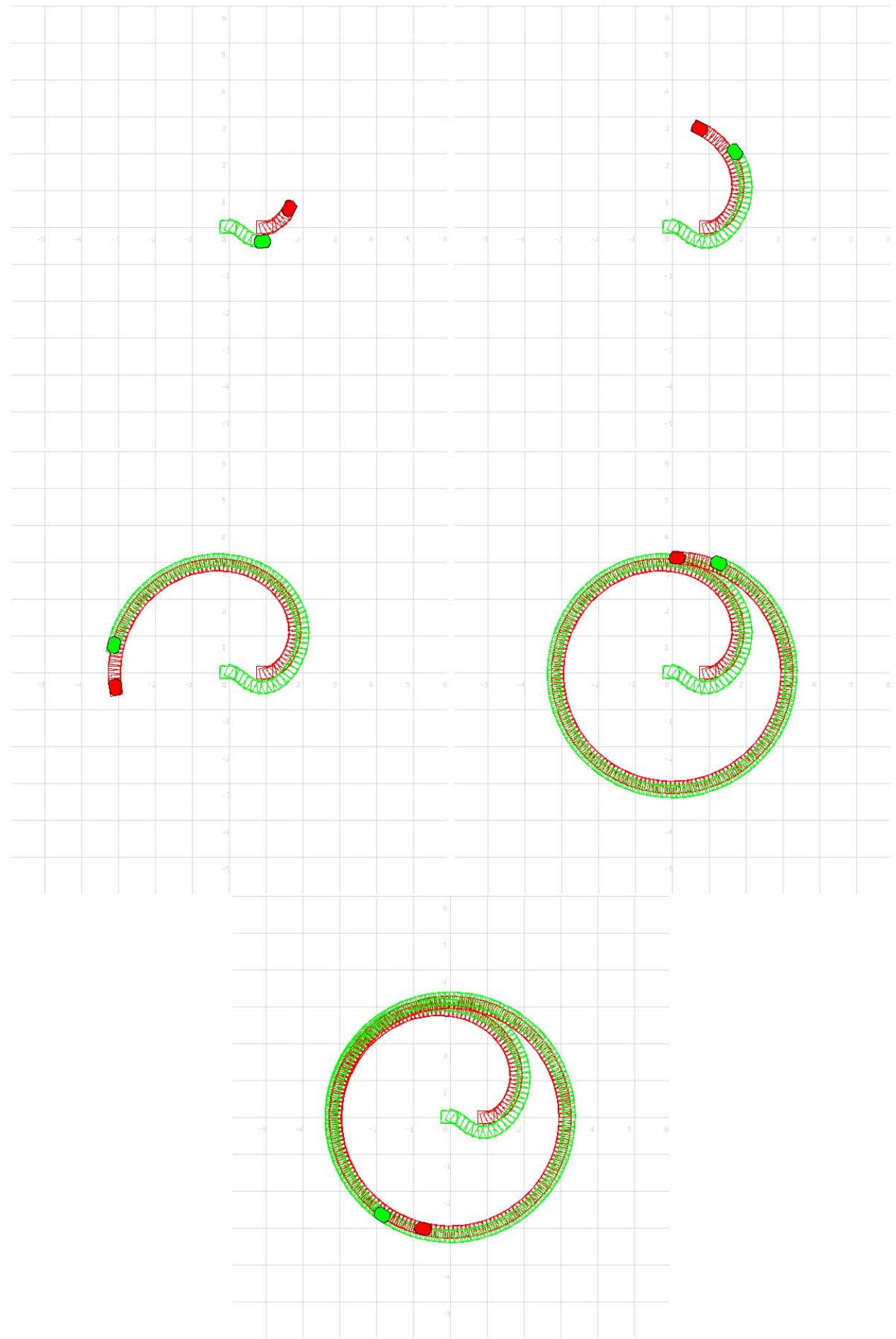

FIGURE 47-Simulation of a leader-follow algorithm, with the leader given the objective to track a circle. The leader is shown in red and the follower in green. The initial position of the robots are near the origin as shown, with the follower 1 meter behind the leader. 


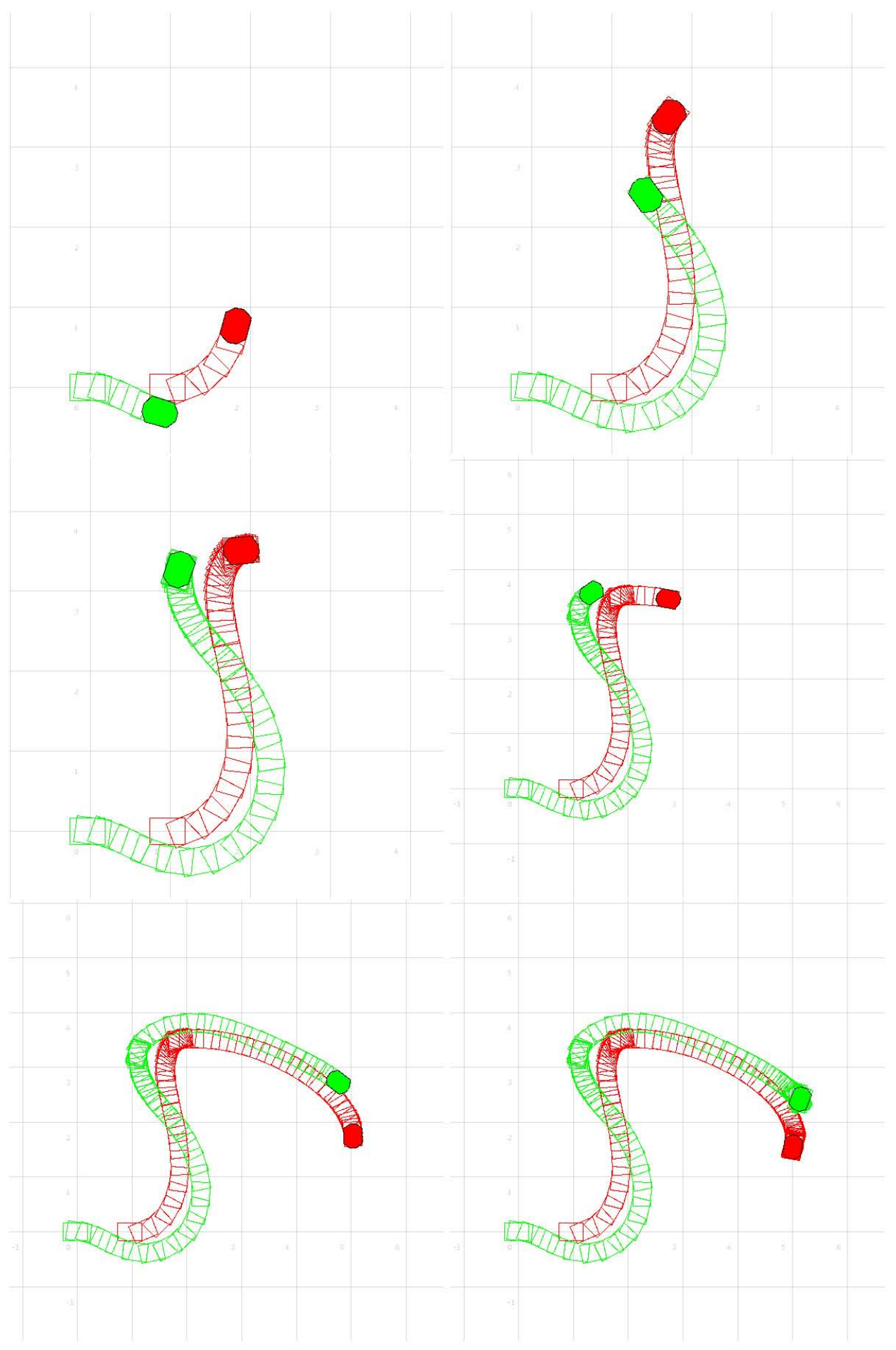

FIGURE 48 - Simulation of a leader-follow algorithm, with the leader given two separate movement commands. The first command was for the leader to go to the pose $\left(2,3.5,0^{\circ}\right)$. Once that was reached, a second command pose was given as $\left(5,1.5-90^{\circ}\right)$. The leader is shown in red and the follower in green. 


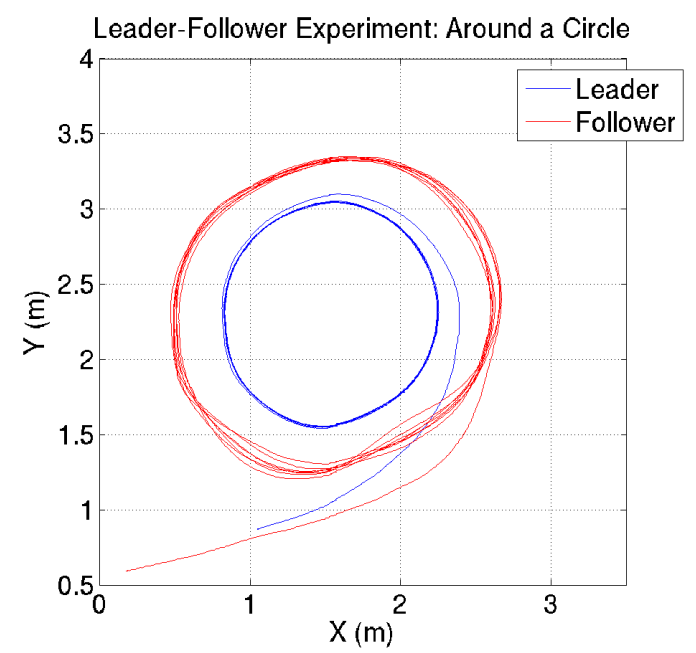

FIGURE 49-Leader-Follow experiment with ER1 robots. The robot was given a circle to track. The initial location of the follower was approximately $0.5 \mathrm{~m}$ behind the leader.

ters. When a 1 meter following distance was used, the follower robot did not have enough room in the testbed.

Figure 49 shows the results of the experiment performed in the testbed. Approximately three minutes of experimental data is shown in the figure. The ER1 robots performed well. Again, the follower moves in a larger circle than the leader. This is more evident in the hardware experiment because a smaller circle was given to the leader. As a result, the follower goal state is cast farther away from the leader's circle. As the radius of the leader circle approaches infinity, the difference between the leader and follower paths approaches zero.

The leader moved in a very consistent circular path in this experiment. Figure 49 shows that the leader repeatedly drove within millimeters of the path. The follower robot was not as consistent as the leader. The red path appears to be a slightly noisier version of the leader's. This behavior was not apparent in the simulator. Unlike the simulator, some level of noise is always present in the hardware system. Noise causes slight variations in the control loop of the leader. The leader's controller does a good job of tracking the circle by making small corrections in angular velocity. These small changes in $\theta$ invoke a larger 


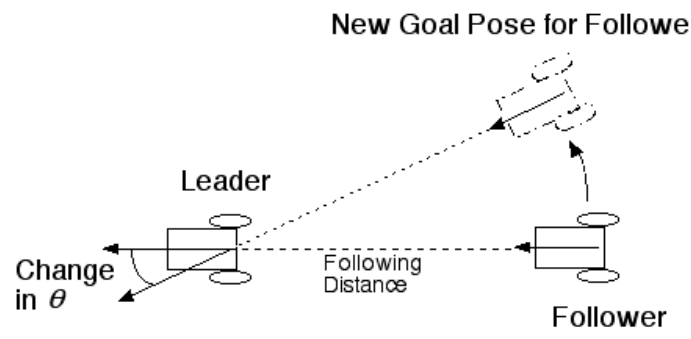

FIGURE 50-Diagram of result of noise in the system. When the leader makes a small change in $\theta$, the goal for the follower makes a larger change. This is the reason that there is more variance in the follower's path.

change in the goal position of the follower. Recall that the follower is tracking the position 0.5 meters directly behind the leader. Figure 50 shows this effect. As the leader rotates slightly, the goal pose of the follower shifts a considerable amount.

The robots depicted here are differential drive. They are constrained to move in the local $x$ direction only. The new goal pose is shifted in the local $y$ direction of the follower. In order to track the new goal, the follower is forced to rotate toward the shifted position. So, the unnoticeable change in the leader's $\theta$ results in a large change in the follower's $\theta$. The leader continues to make these small changes in orientation as it tracks the circle. These small changes are magnified in the follower's path.

This affect is more noticeable when a second follower is added to the experiment. The follower algorithm is designed in a way that allows it to be easily extended. A second instance of the follower program is simply run on a new robot. The new robot tries to track the first follower. In this way, the new robot is unaware of the first leader and vice versa. This puts less burden on the leader robot. Now, the first follower simply communicates its own location to the new follower. Figure 51 shows the results of the experiment. The experiment is exactly the same as the previous, except that a new robot is added to the end of the line formation. The first two robots behave the same as before; moving in a nice formation. The third robot has more difficulty following for two reasons. First, the noise mentioned earlier propagates through the formation, growing as it moves from leader to 


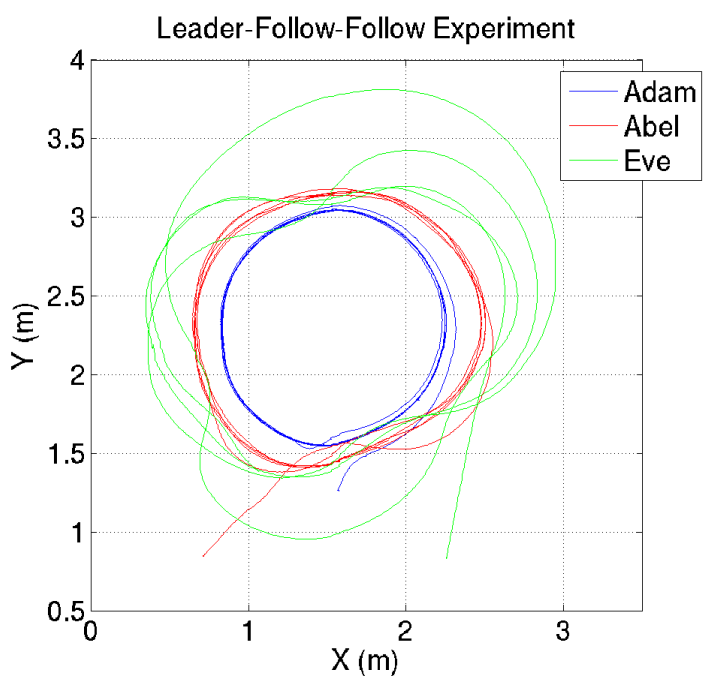

FIGURE 51 - Leader-Follow-Follow experiment with ER1 robots. The leader robot, Adam, shown in red, was given a circle to track. The first follower, Abel, shown in green tried to track Adam. The second follower, Eve, shown in green, tries to follow Abel. The last robot is the tractor-trailer robot design seen earlier.

followers. By the time the last robot receives its goal information, the data is quite noisy, forcing the robot to adjust to a continuously changing radius.

The second reason for the last robot's performance is its configuration. This robot is built with a hinge in its center. The hinge emulates a tractor-trailer. Unlike the other two ER1 robots, this robot is unable to rotate in place. The trailer reduces the degree of mobility by imposing an extra constraint on the robot's kinematics. So, not only does the third robot have a noisier path to follow, it is also constrained more than the other two robots. This explains the second follower's path, shown in green in Fig. 51. 


\title{
CHAPTER VIII EXTENSION TO 3D: A TESTBED DESIGN FOR SMALL-SCALE AERIAL VEHICLES
}

\begin{abstract}
A. Need for a 3D Testbed
Until recently, three dimensional testbeds have been built [5] strictly for outdoor use with large aerial vehicles. They employ expensive GPS and inertial sensors to measure the position of the robots. With the recent availability of small, affordable, aerial vehicles, three dimensional testbeds are being built for indoor use. MIT recently purchased such a testbed from a company called Vicon [6]. Their new testbed uses high-speed, highresolution, infrared cameras to track the position of multiple aerial vehicles. A similar design is presented here, which focuses on using small aerial vehicles in an indoor setting. It is will also use a vision system to track the pose of multiple robots efficiently.
\end{abstract}

\section{B. Analysis \& Design}

1. From $2 \mathrm{D}$ to $3 \mathrm{D}$

In the setup of a typical two dimensional testbed, cameras are mounted overhead and face downward to view the surface. It is customary that the viewing areas of the cameras overlap in order to ease calibration. During calibration, pixels are mapped to a new reference frame usually expressed in distances such as meters. Most of these systems make the assumption that the light rays entering the camera lens are perpendicular to the floor and parallel to each other. A camera located at infinity would receive such light rays; 


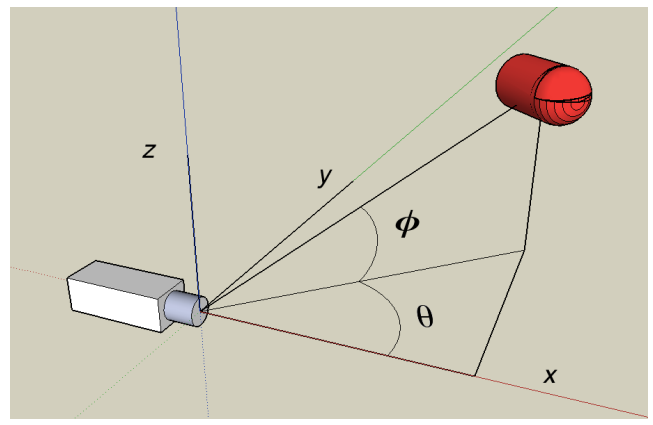

FIGURE 52-Spherical coordinate system of the camera, with the camera at the origin and an aerial vehicle shown in the first octant. A light ray extends from the center of the vehicle to the camera lens. This light ray can be described by its spherical coordinate angles $\theta$ and $\phi$.

however, the testbed cameras are obviously not located at infinity. Even so, this is usually a safe simplification; given that the cameras are mounted a fair distance from the surface of the testbed, and therefore a good distance from the robots themselves. An aerial vehicle will constantly vary its distance from an overhead camera. The closer the vehicle to the camera, the less valid the parallel light ray assumption.

A more accurate model of the incident light rays to the camera involves a change to the spherical coordinate system. Here, in Fig. 52, the camera represents the origin of the system and the light rays approach the lens at varying angles. Each light ray can be modeled with two angles. One, typically denoted $\theta$, is the angle within the $\mathrm{X}-\mathrm{Y}$ plane from the positive $x$-axis. The second, usually $\phi$, is the angle from the $\mathrm{X}$-Y plane.

Now, a camera is a two-dimensional sensor. Thus, in order to measure three dimensions, it is necessary for a robot to be covered by more than one camera at any given time. The difficulty lies in placing the cameras in such a way as to cover each point in the testbed space at least twice. The natural tendency to extending a two-dimensional testbed to three dimensions is to simply place cameras on a wall that look across the testbed. The overhead cameras could measure the $x$ and $y$ variables, while the wall cameras would measure the $z$ position of the vehicle. This approach is limited by another factor of the camera: field of view. With the two-dimensional testbed, the parallel ray assumption ignored the field of view of the cameras. Having done away with this assumption, Figs. 53 and 54 show that the 


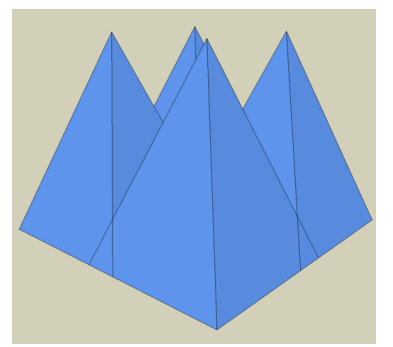

FIGURE 53-Camera coverage of four cameras suspended from the ceiling. Each camera is located at the point of each "pyramid". The pyramids overlap creating this shape which shows the viewable space of the cameras.

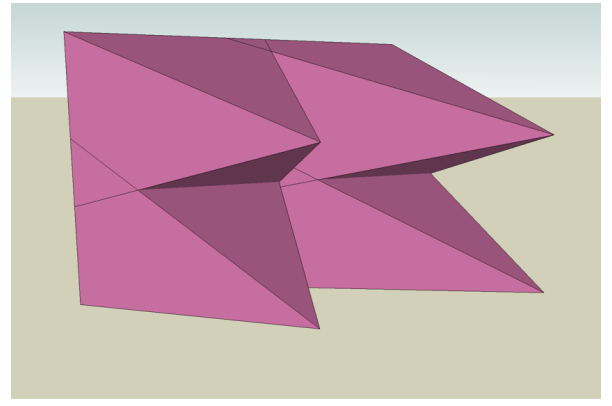

FIGURE 54-Camera coverage of four cameras mounted on a wall. These would be used to capture the $z$ value, or altitude, of a robot. Each camera is located at the tip of the "pyramid" shape which shows the camera's viewable space. Here the viewable spaces overlap slightly.

field of view plays a much larger role in the viewable space of the camera. In Figs. 53 and 54, four cameras are shown, each with a "pyramid" that represents the camera's viewable space. The camera is at the top of the pyramid. Each camera's pyramid overlaps with its neighbor's. Any space not contained by these shapes is not seen by the cameras. With the overhead cameras and the new side wall cameras, there are large, triangular prism-shaped areas that are unseen. If the aerial vehicle climbs too high, the overhead cameras will not be able to see the robot at all. The same is true as the vehicle gets closer to the side wall cameras.

A simple solution would be to add more cameras to cover the vacant area or to move the cameras outside of the testbed, so that there is a larger viewable space. However, this solution does not remedy the problem of the field of view. It also increases the cost of the system, both with hardware and processing power to support the hardware. There is a 


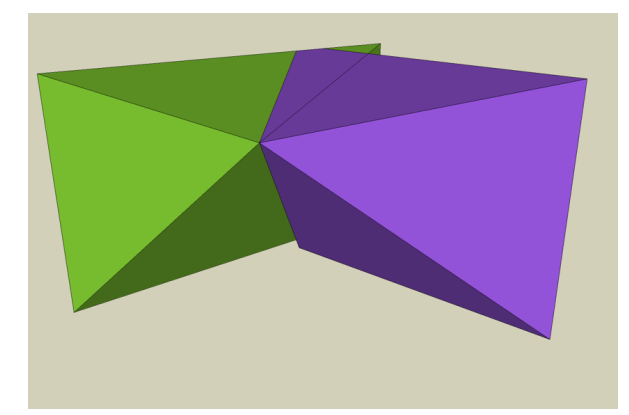

FIGURE 55-This figure shows two cameras mounted very close to each other in an upper corner of the proposed testbed. They are angled downward slightly at the same $\phi$ angle but have different horizontal (or $\theta$ ) angles. The viewable spaces overlap slightly. For clarity, the left camera's viewable space pyramid is a different color than the right camera's pyramid.

simple solution that consolidates the field of view gaps for a continuous region of coverage.

Instead of placing cameras along primary axes, the cameras could be positioned in the corners of the testbed. The cameras could then be oriented to account for the field of views and cover as much continuous area as possible. This is best explained with the help of another figure. In Fig. 55, it is easy to see that two cameras placed in close proximity, but at different orientation angles, cover a large majority of the testbed. Notice also that the region near the ceiling, where an aerial vehicle is most likely to fly, does not have a gap as before. Now, in order to capture an accurate three dimensional pose of the vehicle, it is necessary that at least two cameras can "see" the robot. Seeing little overlap between these two angled cameras, more cameras are obviously needed. That being so, two "mirrored" cameras are added in the adjacent corner in Fig. 56. Now, a large part of the volume can be seen by at least two cameras - enough to get the information needed.

\section{Calculation of the Pose}

When the vehicle of interest is seen by at least two cameras, the pose variables can be calculated using simple geometry. The three dimensional problem can be analyzed in two dimensions for each orthogonal angle $\theta$ and $\phi$. In Fig. 57, an overhead view of the testbed with an object of interest spotted by two arbitrary cameras is shown. The center of 


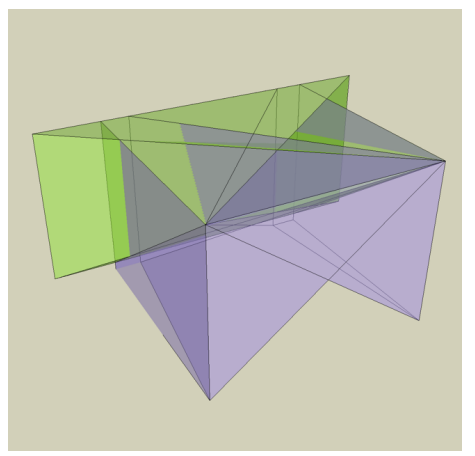

FIGURE 56-The same as Fig. 55, except two more cameras are added to the adjacent corner. The pyramids are shown as semi-transparent to more easily see the total coverage. The entire upper section of the testbed is covered by the cameras.

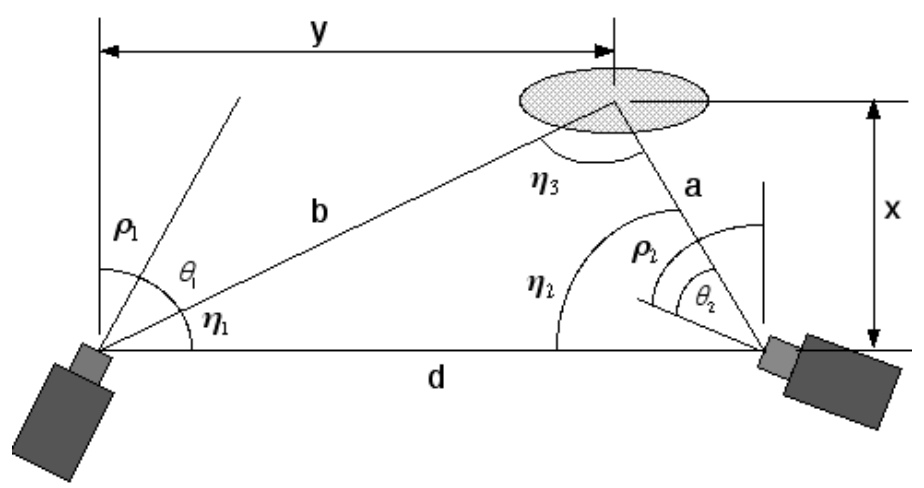

FIGURE 57-An overhead view of two cameras mounted in adjacent corners a distance $d$ from each other, at arbitrary angles $\rho_{1}$ and $\rho_{2}$. The ellipse shown is an aerial vehicle. The cameras are used to measure $\theta_{1}$ and $\theta_{2}$ and triangulate the vehicle's $x$ and $y$ coordinates.

mass of the object will correspond to a $\theta$ angle and a $\phi$ angle for each camera. Taking into account the orientation of each camera, $\rho_{1}$ and $\rho_{2}$, the angle between the intersecting light rays, $\eta_{3}$, can be calculated from (24), (25) and (26).

$$
\begin{gathered}
\eta_{1}=90^{\circ}-\theta_{1}-\rho_{1} \\
\eta_{2}=90^{\circ}+\theta_{2}-\rho_{2} \\
\eta_{3}=-\eta_{2}-\eta_{1}+180^{\circ}
\end{gathered}
$$

The distance between the cameras, $d$, is known. So, using the law of sines, with the definition of the sine ratio, the distance $x$ is found. Equation (27) allows for the other two legs of 


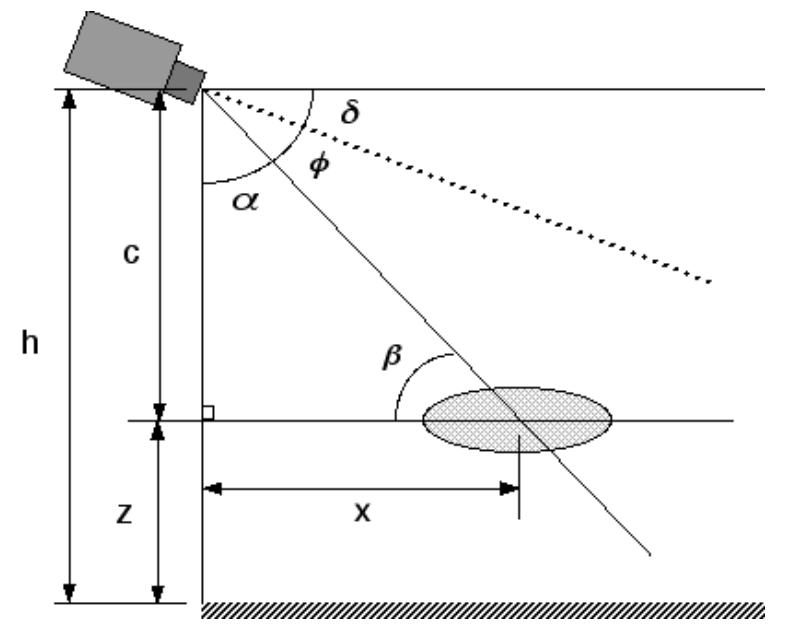

FIGURE 58 - The cameras from Fig. 57 seen from the side. They are both at the same position and downward orientation $\delta$. The ellipse is the aerial vehicle. The cameras both measure the same angle $\phi$, which is then used with the recently found value of $x$ to calculate $z$, the altitude of the vehicle.

the triangle, $a$ and $b$, to be calculated.

$$
\frac{\sin \eta_{1}}{a}=\frac{\sin \eta_{2}}{b}=\frac{\sin \eta_{3}}{d}
$$

Finally, the desired $x$ variable is calculated using the simple definition of the sine ratio.

$$
x=b \sin \eta_{1}=a \sin \eta_{2}
$$

The $y$ component can be calculated just as easily with the cosine ratio.

$$
y=b \cos \eta_{1}=a \cos \eta_{2}
$$

In order to find the altitude, $z$, the side view is examined. Using the information found previously, $x$, and the angle $\phi, z$ can be calculated using the tangent ratio. Fig. 58 shows the side view of the cameras along with the object of interest. The angle from the horizontal to the camera's central axis is denoted as $\delta$. The ray corresponding to the object of interest's center of mass is measured by the camera as $\phi$. From the opposite interior angles of parallel lines theorem, $\beta$ can be defined in (30) as

$$
\beta=|\phi+\delta|
$$


Once $\beta$ is found, the altitude can be calculated with (31).

$$
z=h-x \tan \beta
$$

Therefore, all of the position variables, $x, y$, and $z$, can be calculated using the view of two cameras. This allows an aerial vehicle to move through a large continuous space with a known, three-dimensional position.

A three dimensional, indoor testbed can be used to perform many types of robotics experiments. The size and price tag of aerial vehicles continues to shrink, allowing researchers more access to this type of craft for research. In order to develop new systems and perform reliable experiments, an instrument is needed to accurately measure the movement of aerial vehicles. The vision-based testbed presented here will be able to meet those needs. Aircraft can be tracked easily using the simple techniques that this testbed relies upon. 


\section{CHAPTER IX CONCLUSIONS \& FUTURE WORKS}

\section{A. Conclusions}

This testbed is an enabling technology. It is a tool that can be used for advanced research in mobile robotics. The functions are two-fold. Control algorithms can be developed and then verified with a physical system. Here, a design has been presented for an accurate, real-time multi-robot testbed. Two separate designs for tracking vehicles were demonstrated. The implementation details were presented along with problems met and overcome.

Several difficulties were encountered with the cameras themselves. Trying to match the color output of four different cameras proved to be a challenge. The resulting outputs are close, but certainly not the same. This affected the thresholding of colors. A hardware configuration was found to separate the color levels for easier thresholding.

The camera lens distortion also became a major issue. It caused some of the algorithms to fail when the robots moved between different camera areas. This was eventually rectified by raising the cameras. There is still some distortion present, but not enough to interrupt the tracking.

Once the camera issues were discussed, the performance of the testbed was analyzed for alignment with the design goals of the apparatus. It was found to meet and surpass the original expectations and requirements of the testbed design.

As a demonstration of its function, several applications were explored covering control systems, modeling and autonomous coordinated movement. Several types of con- 
trollers were developed to track circles and move to various poses. The testbed successfully provided position feedback for the mobile robots. It also proved to function much better than the robot's internal odometers as a source of reliable feedback.

Using robust identification algorithms, models for an individual robot were developed. The models presented closely matched the experimental data. The testbed also served well using multiple robots to test coordinated movement controllers in the leader-follow experiments. A method of decentralized control was investigated and verified using actual robot hardware. The algorithm was then extended to a leader-follow-follow formation with the ER1 robots.

Finally, a design was presented to extend the current testbed to three dimensions. This would allow the tracking of not only ground vehicles, but small, indoor, aerial vehicles as well. The experiments presented here could then be applied in three dimensions, allowing for much more advanced algorithms to be developed and verified.

\section{B. Future Works}

Now that the testbed is functional, many types of mobile robot research can be studied. With three robots available, coordinated formation movements can be investigated. Since the robots are heterogeneous in structure, control theories can be tested and verified quickly with different models. The simple controllers presented here can be replaced with much higher level controllers. Optimum and robust controllers can be developed to overcome the problems with the simple PD and PI designs of this thesis.

The leader-follow algorithm can be further extended or enhanced to provide better coordination. Instead of a robot simply tracking an offset distance behind a leader, maybe the follower could use the leader's pose information as "waypoints". In this way, the follower would actually try to follow the exact path of the leader. This could minimize the noise effect that was discussed previously, especially as the number of robots increases.

As far as the $3 \mathrm{D}$ testbed is concerned, only a theoretical design is presented here. 
The next step is to actually construct it. The operation of this tool will be the ultimate test for the validity of the ideas presented here. One issue not addressed in this paper is the tracking of multiple vehicles in three dimensions. Multi-agent research is already popular for ground vehicles and becoming more popular for aerial vehicles as they become more available. The testbed will need the ability to track multiple aircraft.

The proposed 3D testbed will be able to track many vehicles, except in the case of vehicle occlusion. If one vehicle totally or partially blocks another vehicle from being seen by two cameras, the position of the occluded vehicle cannot be measured accurately, if at all. By adding cameras from other points of view and extending the principles presented here, this situation might be avoided in part. However, this does not truly solve the underlying problem.

The applications of this testbed are bound only by the imagination. Many new techniques can be developed with this flexible platform. They can then be verified using physical mobile robots. This ability is an invaluable asset to the advancement of robotics research. Will a robot eventually assist the elderly, or protect soldiers at the next war front? Will a machine search for precious resources on nearby planets? The answer to all of these is yes. The real question is when, but the answer is soon. 


\section{APPENDIX I}

\section{PROGRAM 1: Single Robot Tracking}

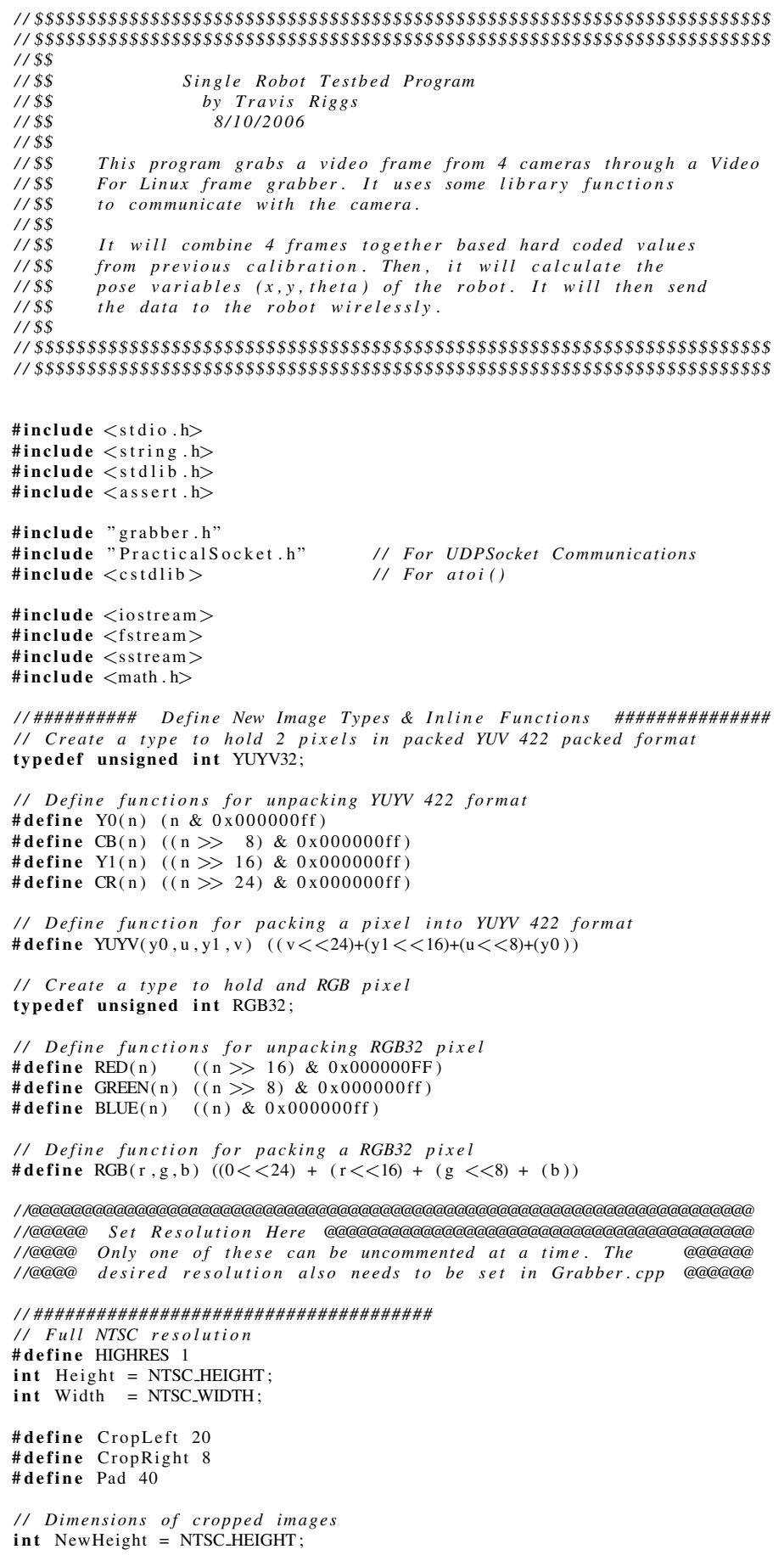


int NewWidth $=$ NTSC_WIDTH - CropLeft - CropRight;

YUYV32 Frame0 [NTSC_HEIGHT*NTSC_WIDTH/2 ];

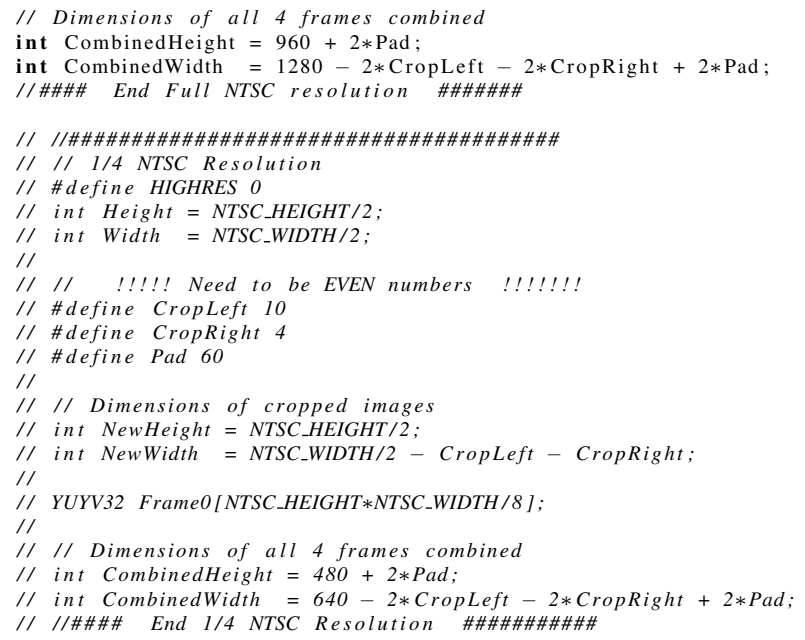

int main(int $\operatorname{argc}, \operatorname{char} * \operatorname{argv}[])$

\{

//@a@a@a@a@a@@a@a@a@a@a@a@a@a@a@a@a@a@a@a@a@a@a@a@a@a@a@a@a@a@a@a@a

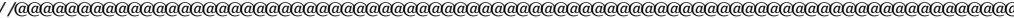

int Debug $=1 ; \quad / /$ Debug $=1$, then only perform one iteration and print debug info

int $w, h$

int $\mathrm{i}, \mathrm{j}$;

int $\mathrm{f}, \mathrm{g}$;

int color

int iter;

int CaptureIterations: $/ /$ Number of measurements to take (command line argument)

int FoundRobot $=0 ; \quad / /$ Flag to indicate program found the robot

char *name; $\quad$ // Name to store picture files as ppm images

char *Camera; $\quad$ // Store device name of camera

int $\mathrm{R}, \mathrm{G}, \mathrm{B}$; // Actual pixel values from frame in RGB space

int $\mathrm{Y}, \mathrm{Cb}, \mathrm{Cr} ; \quad / /$ Calculated pixel values in $\mathrm{YCbCr}$ (YUV) space

YUYV32 pixels; /I Structure to hold packed YUYV 422 pixels (2 pixels)

RGB32 rgbpixel;

// Array to store combined frames for searching

YUYV32 Combined [ ( CombinedHeight $) *($ CombinedWidth $) / 2$ ]

// Array to store debugging image

RGB32 ThreshPic [( CombinedHeight)*(CombinedWidth)];

//========= Center of Mass Calculation Variables for each Color

double RedCenterX = 0

double RedCenterY $=0$

double RedMass = 0 ;

int MinRedMass $=40$

if $($ HIGHRES $==1)$

\{ \}

MinRedMass = $300 ;$

double BlueCenterX $=0$;

double BlueCenterY $=0$;

double BlueMass $=0$;

$/ /=========$ Pose Variables of the Robot

double RobotX; I/ X position of robot (pixels)

double RobotY; $\quad / / Y$ position of robot (pixels)

double RobotTheta; // Theta of Robot (degrees)

$/ /=========$ Conversion Variables

// Calibration Factors to convert pixels to meters

double YPixelsPerMeter $=128.62487 ; / /$ Pixels per meter in the $Y$ direction

double XPixelsPerMeter $=133.98229 ; / /$ Pixels per meter in the $X$ direction

if $($ HIGHRES $==1)$

\{

YPixelsPerMeter $=2 *$ YPixelsPerMeter

XPixelsPerMeter $=2 *$ XPixelsPerMeter; 
double RobotXMeters; // X Position of the robot in meters

double RobotYMeters; // Y Position of the robot in meters

// Number of pixels to skip when searching for red circles

int SkipHoriz $=48$; // This number needs to be a multiple of 4 ... actually twice the \# to skip

int SkipVert $=24$; // This is the actual number to skip in the vertical direction

if $($ HIGHRES $==1)$

\{

KkipHoriz $=2 *$ SkipHoriz

\}

SkipVert $=2 *$ SkipVert

// Number of pixels for search box around a discovered Red pixel

// !!!!! Need to be EVEN numbers !!!!!!!!

int SearchBox $=60 ; \quad / / 16$ will create $a 2 * 16+1 \times 2 * 16+1$ or $33 \times 33$ search box

if $($ HIGHRES $==1)$

\{

\}

SearchBox $=2 *$ SearchBox;

$/ /========$ Image Loop Variables

Need to be EVEN numbers !1!!!!!

int $\mathrm{StartOffsetX}=\mathrm{Pad}+2 ; / /$ Loop variables for searching combined frames

int StartOffsetY $=\mathrm{Pad}+2 ; \quad / /$ We don't want to waste time searching the black

int EndOffset $\mathrm{X}=500 ; \quad / /$ border (padding) of the combined image.

int EndOffsetY $=614 ; \quad / / \quad$ These were found empirically.

if $($ HIGHRES $==1)$

\{

EndOffset $X=2 *$ EndOffset $X$ \} EndOffset $Y=2 *$ EndOffset $Y$;

// Dimensions from Calibration to stitch frames together:

II Initialize Calibration Variables. When I first calibrated these values, I did it

I/ at the floor level, but the hats sit about 14" above the floor. Due to the cone

// angle, the floor level calibrations were cropping pieces of the hat off when the

// robot would move from one camera to another on the floor. Here I'm using all the

// true pixels (I cropped out the artifacts) I can get to ensure an accurate measure-

// -ment of the robots pose. Be careful if you change these, because each cameras uses

// these values differently in the "for" loops below.

// $X$ and $Y$ point of center of testbed (in the lower right corner of Cam O)

int $\mathrm{XCal0}=209 ; \quad / / 240$;

int $\mathrm{YCal}=280 ; \quad / / 320-$ CropLeft - CropRight -4 ;

if $($ HIGHRES $==1)$

\{

$\mathrm{XCal0}=418 ; \quad / / 480 ;$ \}

YCal0 $=561 ; \quad / / 640-$ CropLeft - CropRight $-8 ;$

// X\& Y point of center of testbed (in lower left corner of Cam 1)

int $\mathrm{XCal1}=197 ; \quad / / 240$

int $\mathrm{YCal1}=21 ; \quad / / 10$;

if $($ HIGHRES $==1)$

\{

$\mathrm{XCal1}=395 ; \quad / / 480 ;$

\}

$$
\text { YCall }=42 ; \quad / / 20 ;
$$

// X\&Y point of center of testbed (in upper right corner of Cam 2)

int $\mathrm{XCal} 2=29 ; \quad / / 0$;

int $\mathrm{YCa} 2=280 ; \quad / / 320-$ CropLeft - CropRight -2 ;

if $($ HIGHRES $==1)$

\{

$\mathrm{XCal} 2=58 ; \quad / / 0$ \}

$\mathrm{YCa} 2=561 ; \quad / / 640-$ CropLeft - CropRight $-4 ;$

// X\&Y point of center of testbed (in upper left corner of Cam 3)

int $\mathrm{XCa} 3=30 ; \quad / / 2$;

int $\mathrm{YCal} 3=26 ; \quad / / 10$

if $($ HIGHRES $==1)$

\{

$\mathrm{XCal} 3=60 ; \quad / / 4 ;$

\}

$\mathrm{YCal3}=52 ; \quad / / 20 ;$

//========== Thresholding Variables

int YThreshTable[256]; // Y Lookup Table to do fast constant thresholding

int CbThreshTable[256]; // Cb Lookup Table to do fast constant thresholding

int CrThreshTable[256]; // Cr Lookup Table to do fast constant thresholding

int ThresholdResult;

// Holds result of bitwise AND operations for thresholding

int WhiteMask $=0 \times 01 ; / /$ Masks to access color lookup result

int RedMask $=0 \times 02$;

int BlueMask $=0 \times 04$;

int GreenMask $=0 \times 08$;

int BlackMask $=0 \times 10$; 


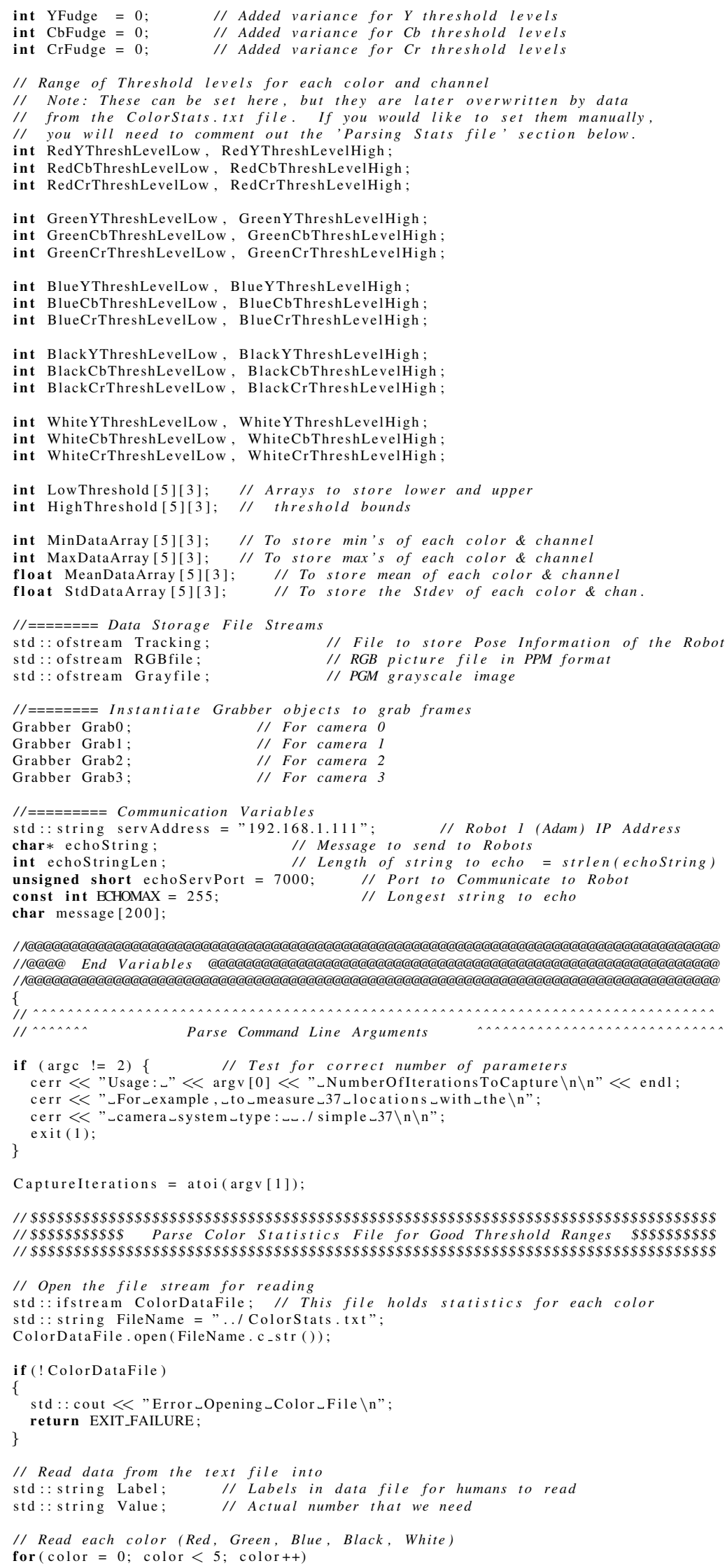




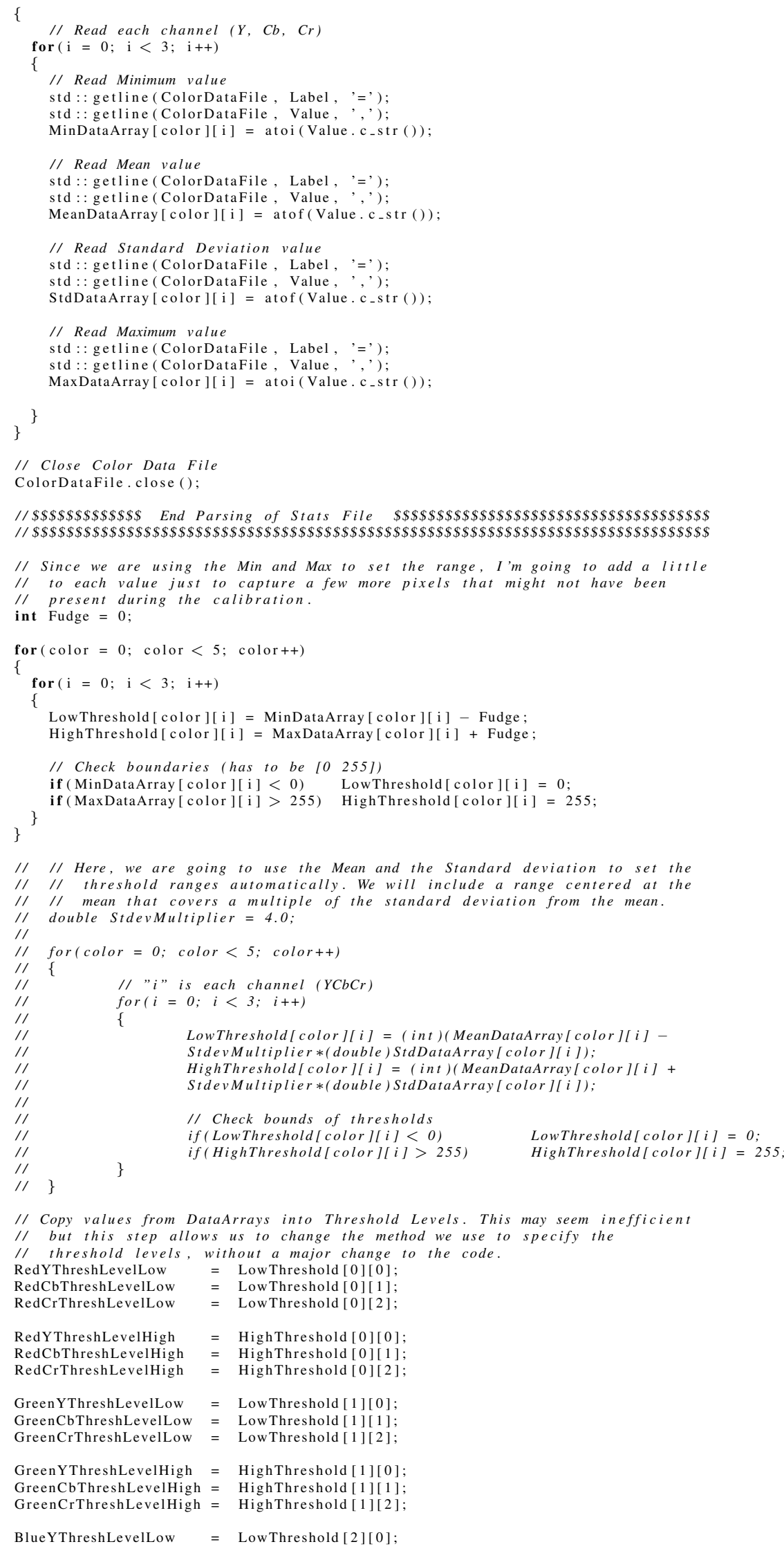

$=$ LowThreshold [2][0]: 


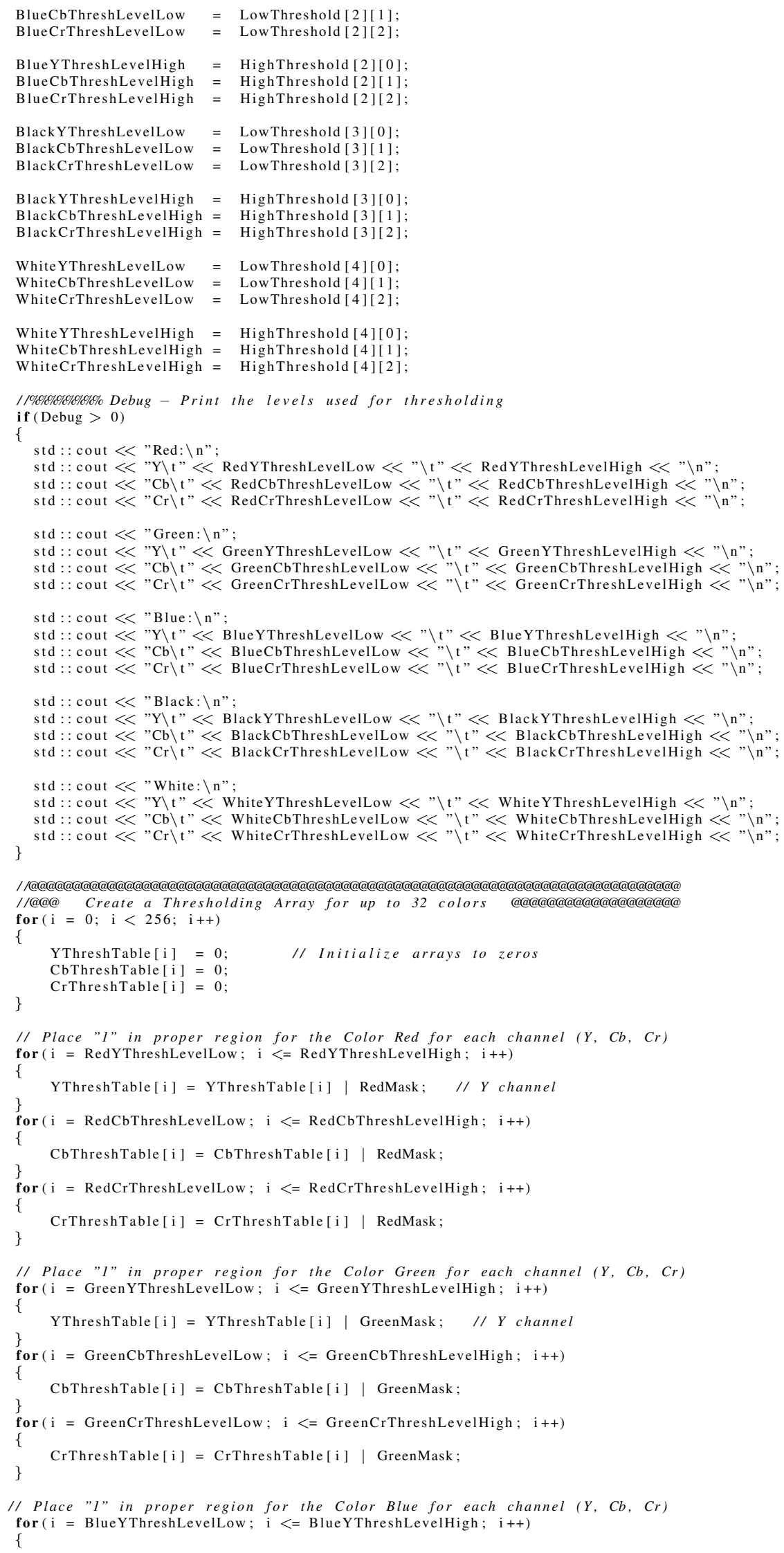




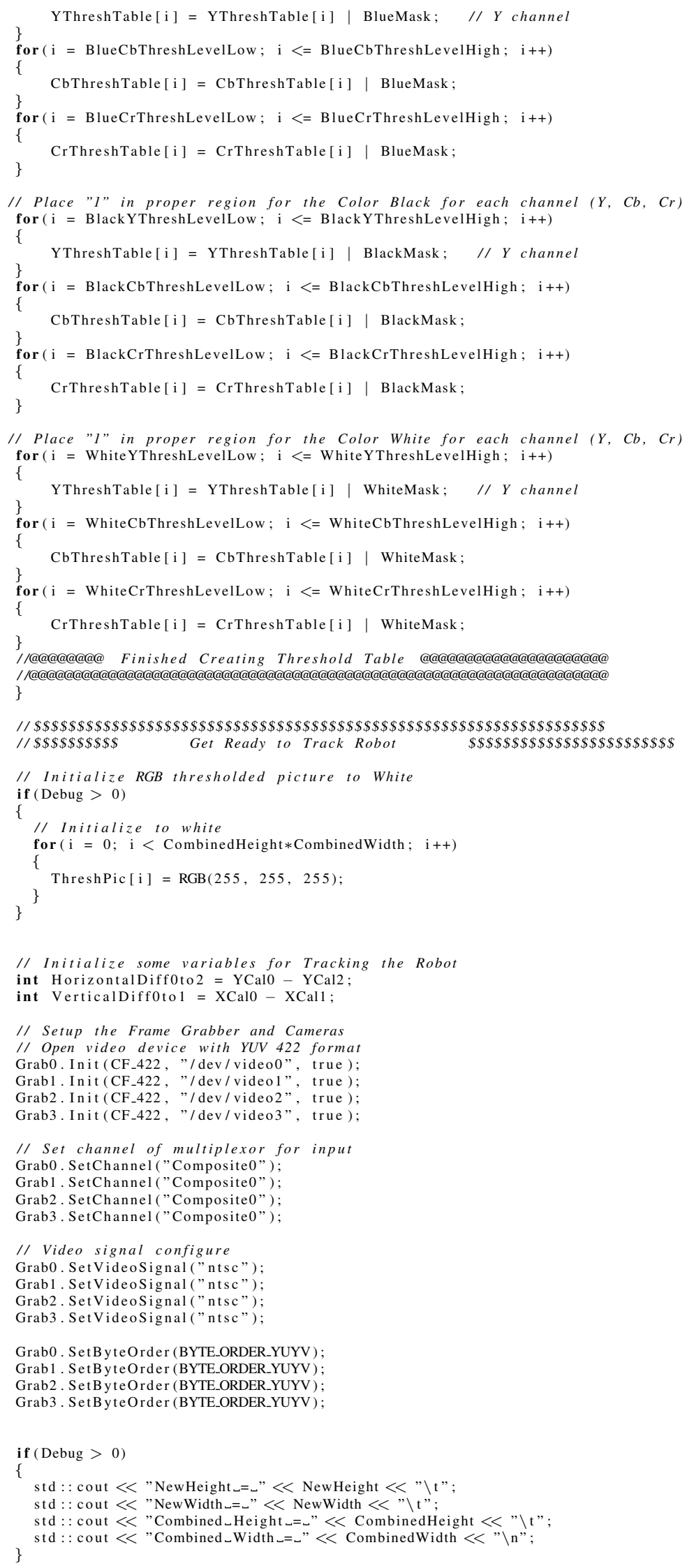




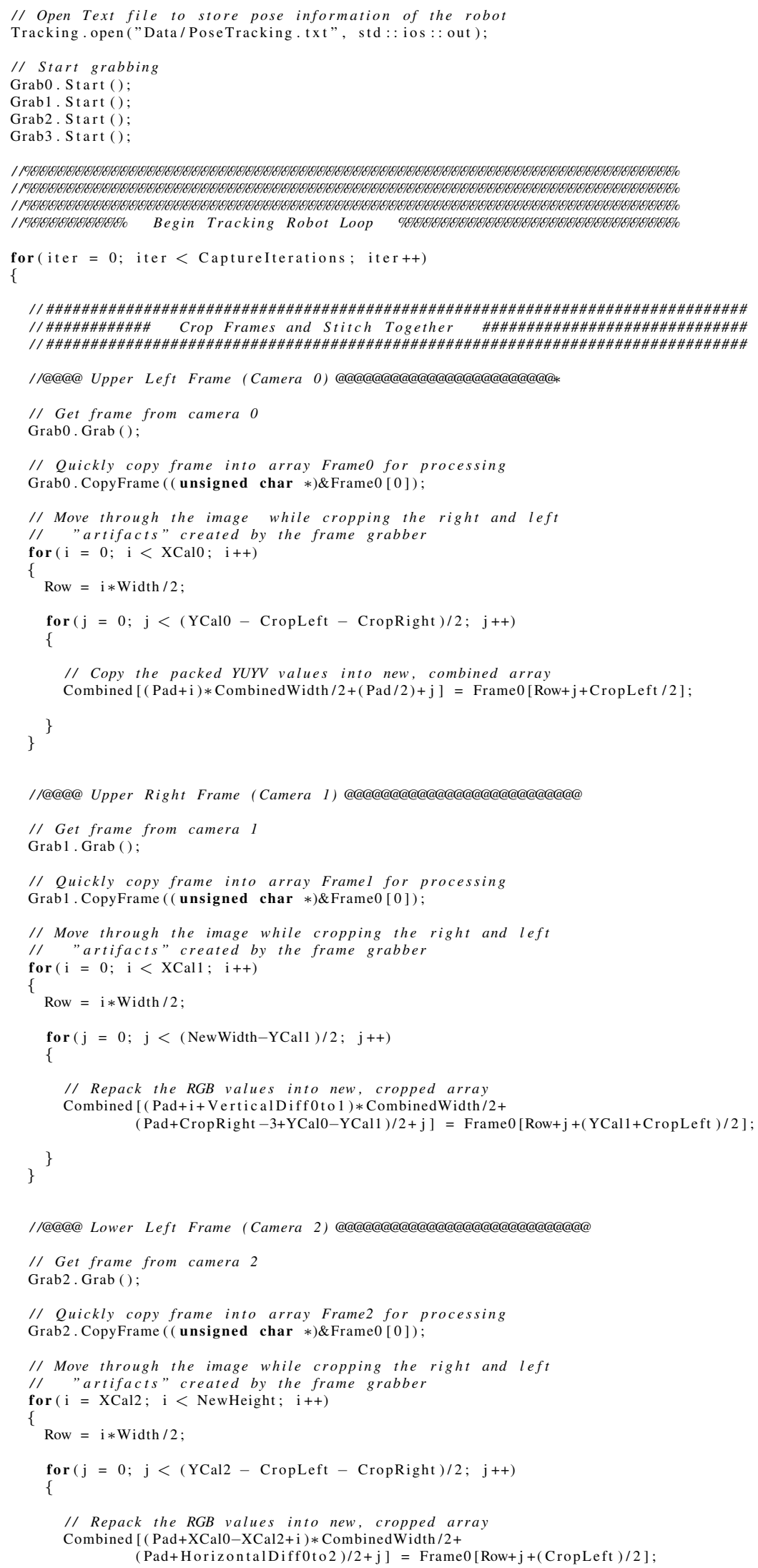


// Get frame from camera 3

Grab3. Grab ();

// Quickly copy frame into array Frame3 for processing

Grab3. CopyFrame (( unsigned char *)\&Frame0 $[0])$;

// Move through the image while cropping the right and left

// "artifacts" created by the frame grabber

for $(\mathrm{i}=\mathrm{XCal3} ; \mathrm{i}<\mathrm{NewHeight;} \mathrm{i}++)$

Row $=\mathrm{i} *$ Width $/ 2$;

for $(j=0 ; j<($ NewWidth - YCal3 $) / 2 ; j++)$

// Repack the RGB values into new, cropped array

Combined $[($ Pad + XCal0 $-X C a 13+\mathrm{i}) *$ CombinedWidth $/ 2+($ Pad + HorizontalDiff 0 to $2+$ CropLeft $+\mathrm{YCal} 0-\mathrm{YCal} 3) / 2+\mathrm{j}]=$ Frame $0[$ Row $+\mathrm{j}+(\mathrm{YCal} 3+\mathrm{CropLeft}) / 2]$

\}

//\#\#\#\#\#\#\#\#\#\#\# End Cropping \& Stitching \#\#\#\#\#\#\#\#\#\#\#\#\#\#\#\#\#\#\#\#\#\#\#\#\#\#\#\#\#\#\#\#

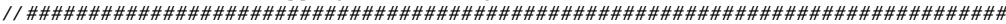

//\#\#\#\#\#\# Save the Combined Image as a Grayscale PGM

// Note: This should really be converted to a function, but I was

// unsuccessful at accomplishing this because of scope issues.

if ( Debug $>0)$

// Open file stream for write operations to save PGM fil

Grayfile .open ("Frames/combined.pgm", std :: ios :: out);

// Write Header for file

Grayfile $<<$ "P2" $<<$ std : : endl ;

Grayfile $<<$ CombinedWidth $<<,-"<<$ CombinedHeight $<<" \backslash$ n";

Grayfile $<<255 "<<$ std : : endl;

// Copy Y pixels into file

for $(\mathrm{i}=0 ; \mathrm{i}<$ CombinedHeight $; \mathrm{i}++)$

\{

for $(j=0 ; j<$ CombinedWidth $; j=j+2)$

// Get 2 packed pixels from image

pixels $=$ Combined $[\mathrm{i} *$ CombinedWidth $/ 2+\mathrm{j} / 2]$;

// Extract first intensity value

$\mathrm{Y}=\mathrm{Y0}($ pixels $)$

// Write to file

Grayfile $<<\mathrm{Y}<<$ ",

// Extract 2 nd intenisty value

$\mathrm{Y}=\mathrm{Y} 1($ pixels $)$;

// Write to file

Grayfile $<<$ Y $<<$ ",

// Close file

Grayfile.close ():

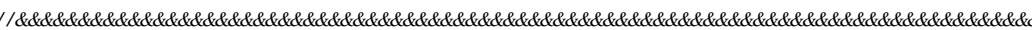

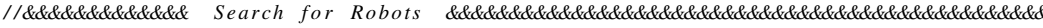

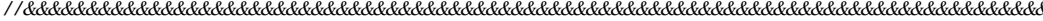

// Initialize Tracking Variables

BlueCenterX $=0$;

BlueCenterY $=0$

BlueMass $=0$

RedCenterX $=0$

RedCenterY $=0$

RedMass $\quad=0$;

// Clear search flag

FoundRobot $=0$

//1a@a@a@a@a@a@a@a@a@a@a@a@a@a@a@a@a@a@a@a@a@a@a@a@a@a@a 1/@@@Search for Red Rectangle in Combined Image @@@@@@@@@@@

// Begin a sparse search of the testbed

for $(\mathrm{i}=$ StartOffsetX $; \mathrm{i}<$ EndOffetX; $\mathrm{i}=\mathrm{i}+$ SkipVert $)$

\{

if $($ Debug $>4)$ 


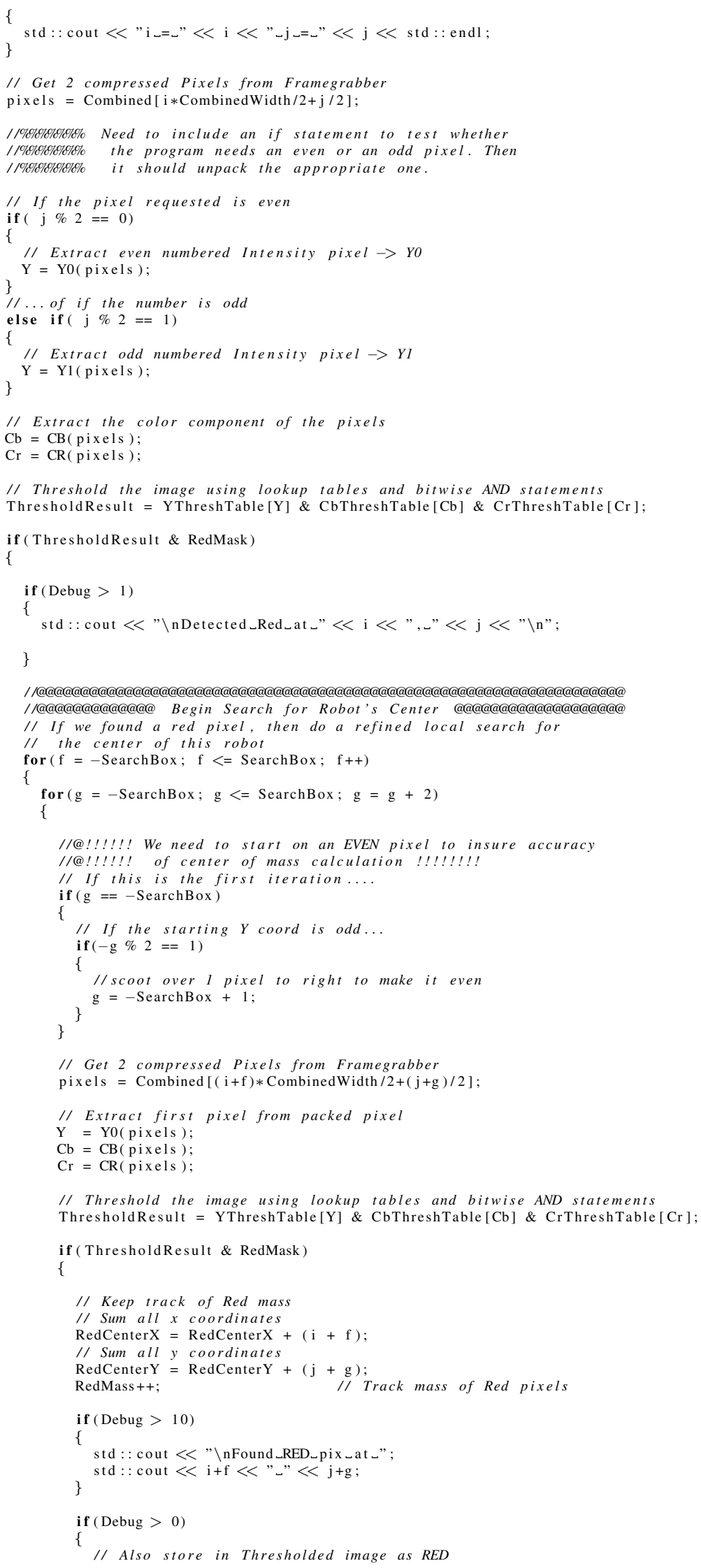


ThreshPic $[(\mathrm{i}+\mathrm{f}) *$ CombinedWidth $+(\mathrm{j}+\mathrm{g})]=\operatorname{RGB}(255,0,0)$;

\}

else if(ThresholdResult \& BlueMask)

\{

// Keep track of Blue mass

// Sum all $x$ coordinates

BlueCenterX $=$ BlueCenterX $+(\mathrm{i}+\mathrm{f})$

// Sum all y coordinates

BlueCenter $\mathrm{Y}=$ BlueCenterY $+(\mathrm{j}+\mathrm{g})$

BlueMass++; $\quad$ // Track mass of Blue pixels

// Store thresholded result as pure YUYV Blue Pixels

Combined $[(\mathrm{i}+\mathrm{f}) *$ CombinedWidth $/ 2+(\mathrm{j}+\mathrm{g}) / 2]=\operatorname{YUYV}(76,255,76,85)$;

if $($ Debug $>10)$

std : : cout $<<" \backslash$ nFound_BLUE_pix _at _ $"$

\} std : : cout $<$ i + f $<<", "<<+$ g;

if ( Debug $>0)$

\{

// Also store in Thresholded image as BLUE \} \}

ThreshPic $[(\mathrm{i}+\mathrm{f}) *$ CombinedWidth $+(\mathrm{j}+\mathrm{g})]=\operatorname{RGB}(0,0,255)$;

if ( Debug $>0)$

\{

// Pixel was unclassified, so set to Black

\}

ThreshPic $[(\mathrm{i}+\mathrm{f}) *$ CombinedWidth $+(\mathrm{j}+\mathrm{g})]=\operatorname{RGB}(0,0,0)$;

// Extract second pixel from packed pixel

$\mathrm{Y}=\mathrm{Y} 1($ pixels $)$;

$\mathrm{Cb}=\mathrm{CB}($ pixels $) ;$

$\mathrm{Cr}=\mathrm{CR}($ pixels $)$;

// Threshold the image using lookup tables and bitwise AND statements ThresholdResult = YThreshTable $[\mathrm{Y}] \&$ CbThreshTable $[\mathrm{Cb}] \&$ CrThreshTable $[\mathrm{Cr}]$;

if (ThresholdResult \& RedMask)

\{

// Keep track of Red mass

// Sum all $x$ coordinates

RedCenterX $=$ RedCenterX $+(\mathrm{i}+\mathrm{f})$

/ Sum all y coordinates (remember to add 1 b/c it's 2 nd pixel)

RedCenterY $=$ RedCenterY $+(\mathrm{j}+\mathrm{g}+1)$;

RedMass++; // Track mass of Red pixels

// Store thresholded result as pure YUYV Red Pixels

Combined $[(\mathrm{i}+\mathrm{f}) *$ CombinedWidth $/ 2+(\mathrm{j}+\mathrm{g}) / 2]=\operatorname{YUYV}(76,85,76,255)$

// For fastest execution

if $($ Debug $==0)$

\{

continue ;

if $($ Debug $>10)$

\{

std : : cout $<<" \backslash$ nFound $\lrcorner$ RED $\lrcorner$ pix $\lrcorner$ at $\lrcorner " ;$

\}

std: : cout $<$ i $+\mathrm{f}<<, ",<<j+g+1$;

if $($ Debug $>0)$

\{

// Also store in Thresholded image as RED

\}

ThreshPic $[(\mathrm{i}+\mathrm{f}) *$ CombinedWidth $+(\mathrm{j}+\mathrm{g}+1)]=\operatorname{RGB}(255,0,0)$;

else if(ThresholdResult \& BlueMask)

\{

// Keep track of Blue mass

// Sum all $x$ coordinates

BlueCenterX $=$ BlueCenterX $+(\mathrm{i}+\mathrm{f})$;

// Sum all y coordinates (remember to add $1 \mathrm{~b} / \mathrm{c}$ it's 2 nd pixel)

BlueCenterY $=$ BlueCenterY $+(\mathrm{j}+\mathrm{g}+1)$;

BlueMass++; $\quad / /$ Track mass of Blue pixels

// Store thresholded result as pure YUYV Blue Pixels

Combined $[(\mathrm{i}+\mathrm{f}) *$ CombinedWidth $/ 2+(\mathrm{j}+\mathrm{g}) / 2]=\operatorname{YUYV}(76,255,76,85)$;

if ( Debug $>10)$

\{ 


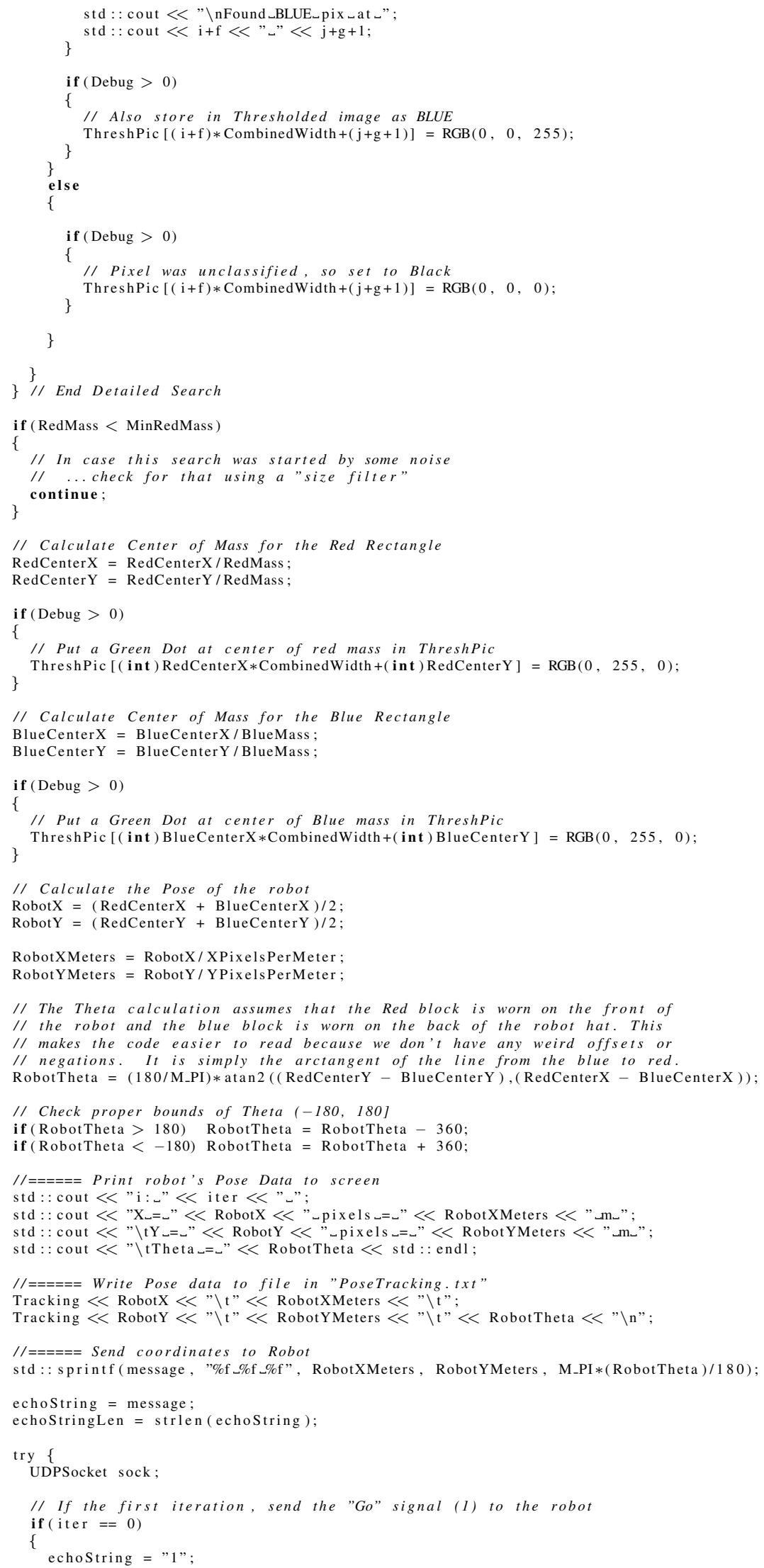


\{

// Open file stream for write operations to save PGM file RGBfile.open("Frames/threshpic.ppm", std:: ios :: out);

// Write Header for file

RGBfile $<<$ "P3" << std : endl;

RGBfile $<<$ CombinedWidth $<<"$ " $<<$ CombinedHeight $<<$ " \n";

RGBfile $<$ " 255 " $<$ std:: endl

// Copy Y pixels into file

for $(\mathrm{i}=0 ; \mathrm{i}<$ CombinedHeight $; \mathrm{i}++)$

for $(j=0 ; j<$ CombinedWidth $; j++)$

// Get 2 packed pixels from image

rgbpixel = ThreshPic [i*CombinedWidth $+\mathrm{j}]$;

// Extract channel values

$\mathrm{R}=\operatorname{RED}(\mathrm{rgbpixel})$

$\mathrm{G}=\operatorname{GREEN}(\mathrm{rgbpixel})$;

$\mathrm{B}=\operatorname{BLUE}($ rgbpixel $)$;

// Write to file

RGBfile $<$ R $<<$, " $<$ G $<<$ ", $<<$ B $<<$ ",",

\}

// Close file

RGBfile. close ()

\}

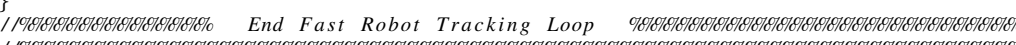

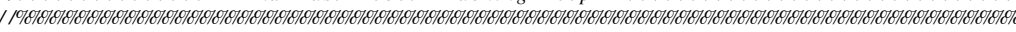

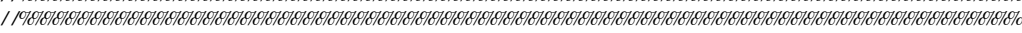

// Stop Frame Grabber

Grab0. Stop ();

Grab1. Stop ():

Grab2. Stop ();

Grab3. Stop ();

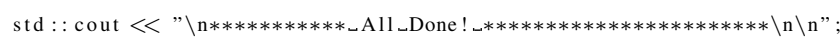

// Close Pose Tracking Text file

Tracking. close ();

return 0 ; 


\section{APPENDIX II}

\section{PROGRAM 2: Multi-Robot Tracking}

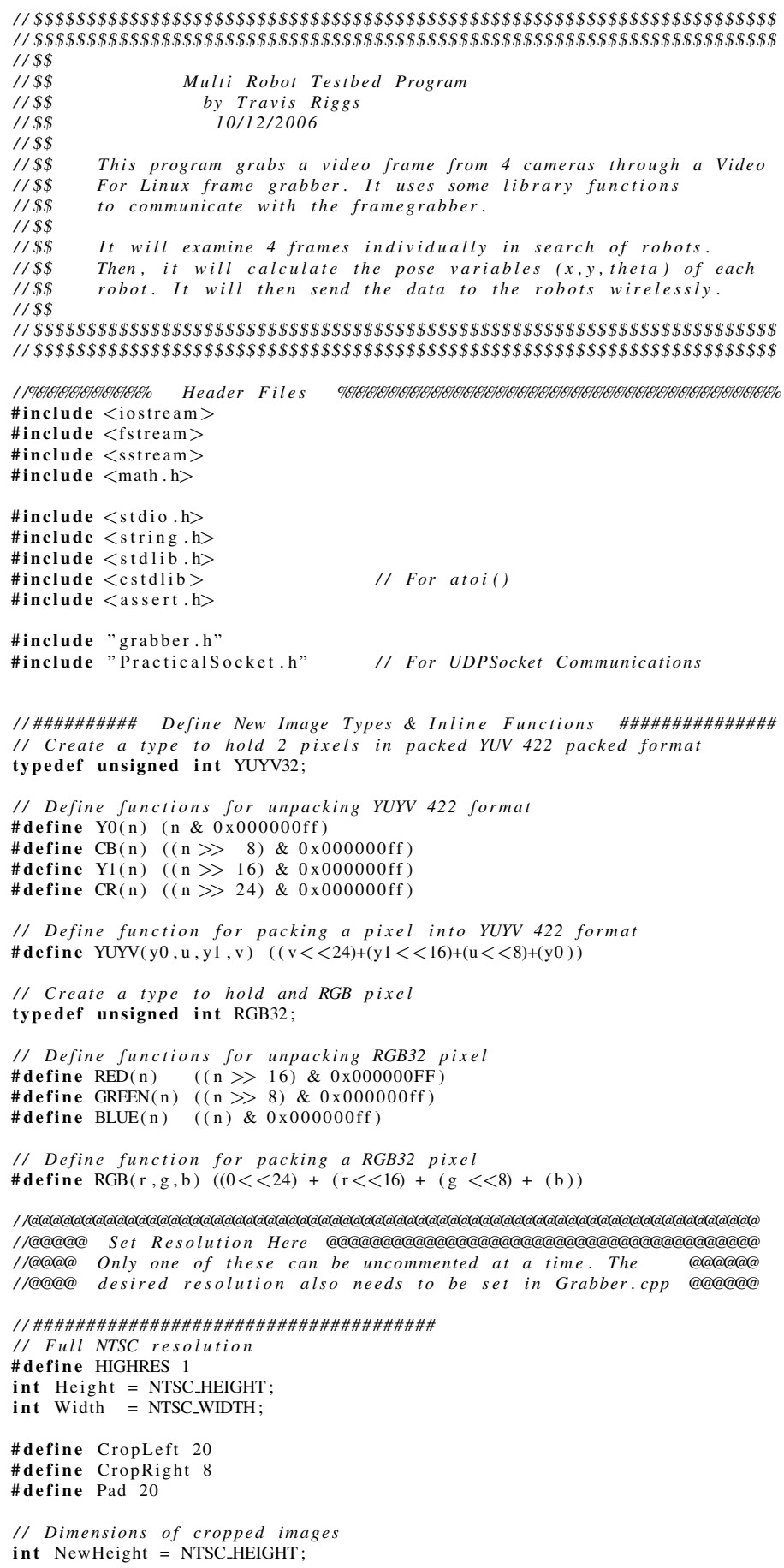


int NewWidth $=$ NTSC_WIDTH - CropLeft - CropRight;

YUYV32 Frame [NTSC_HEIGHT $*$ NTSC_WIDTH / 2 ];

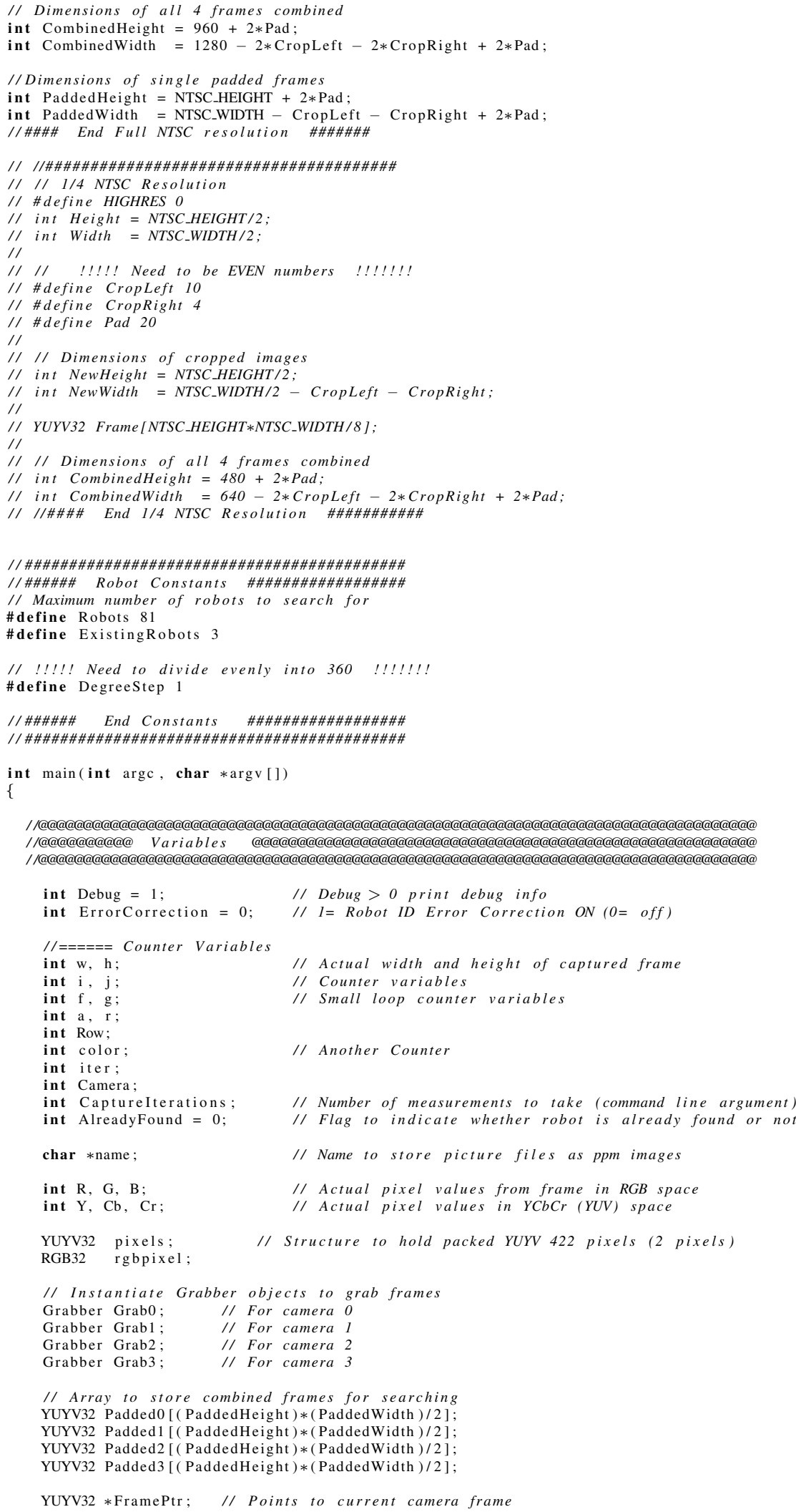




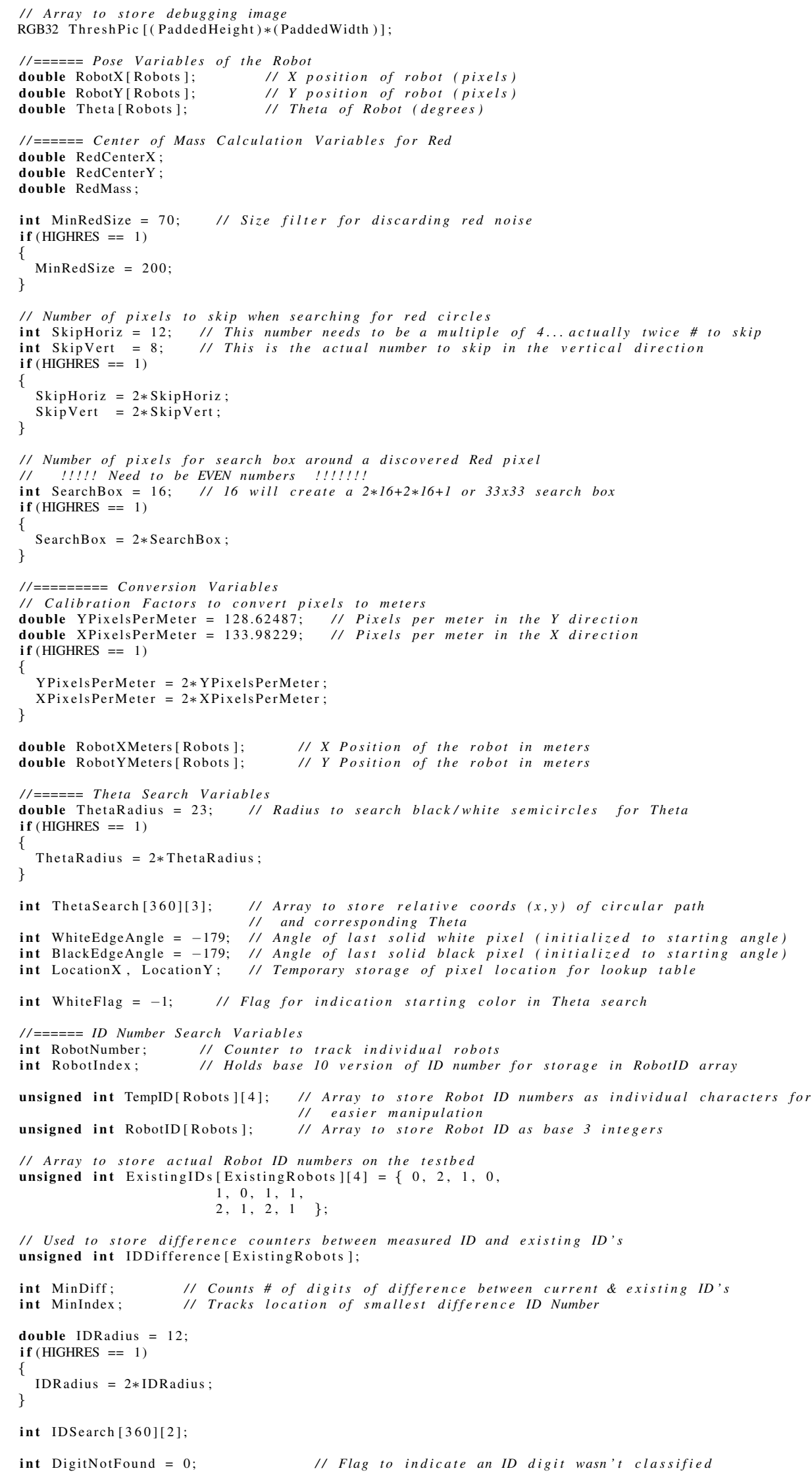




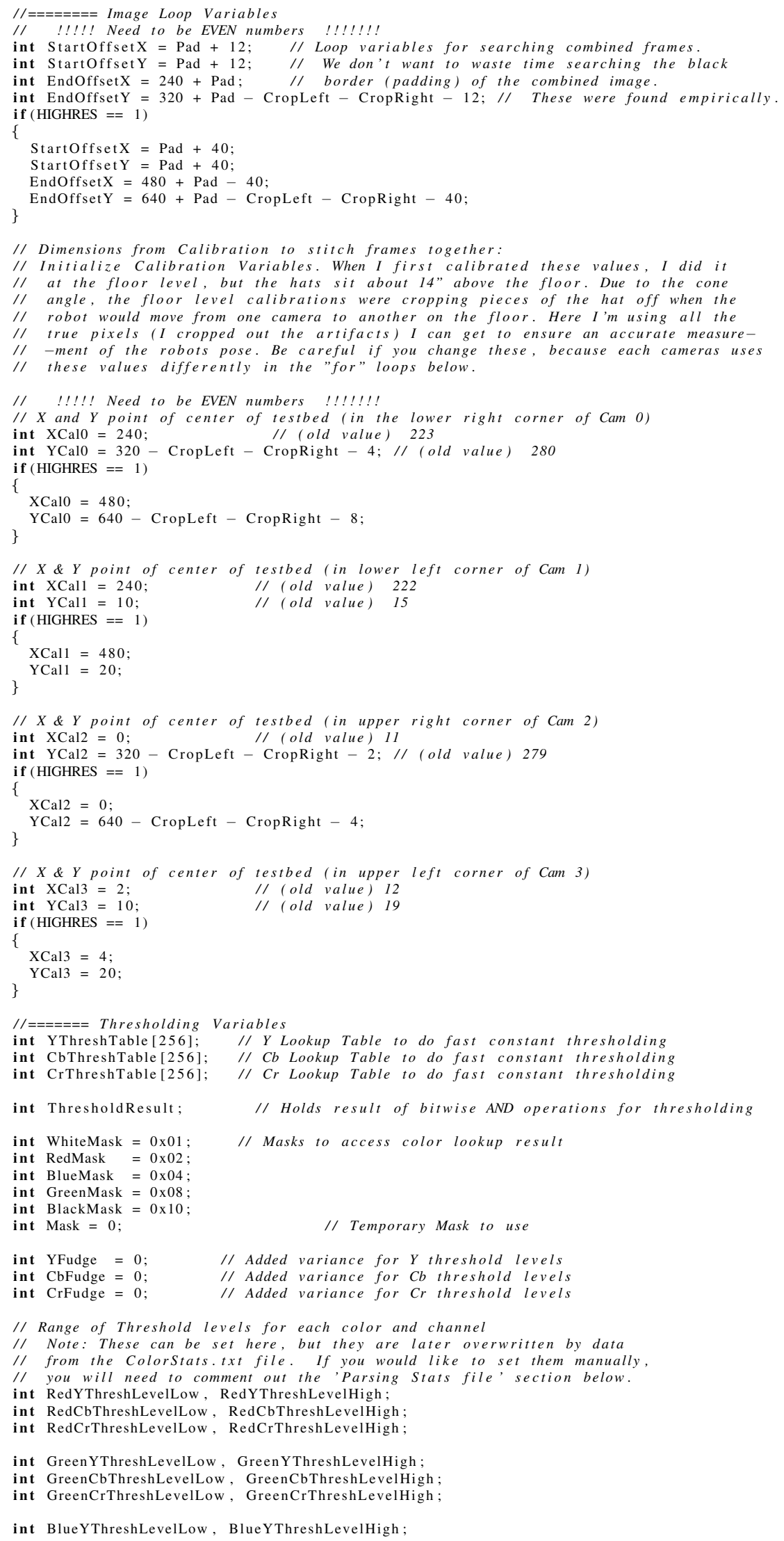




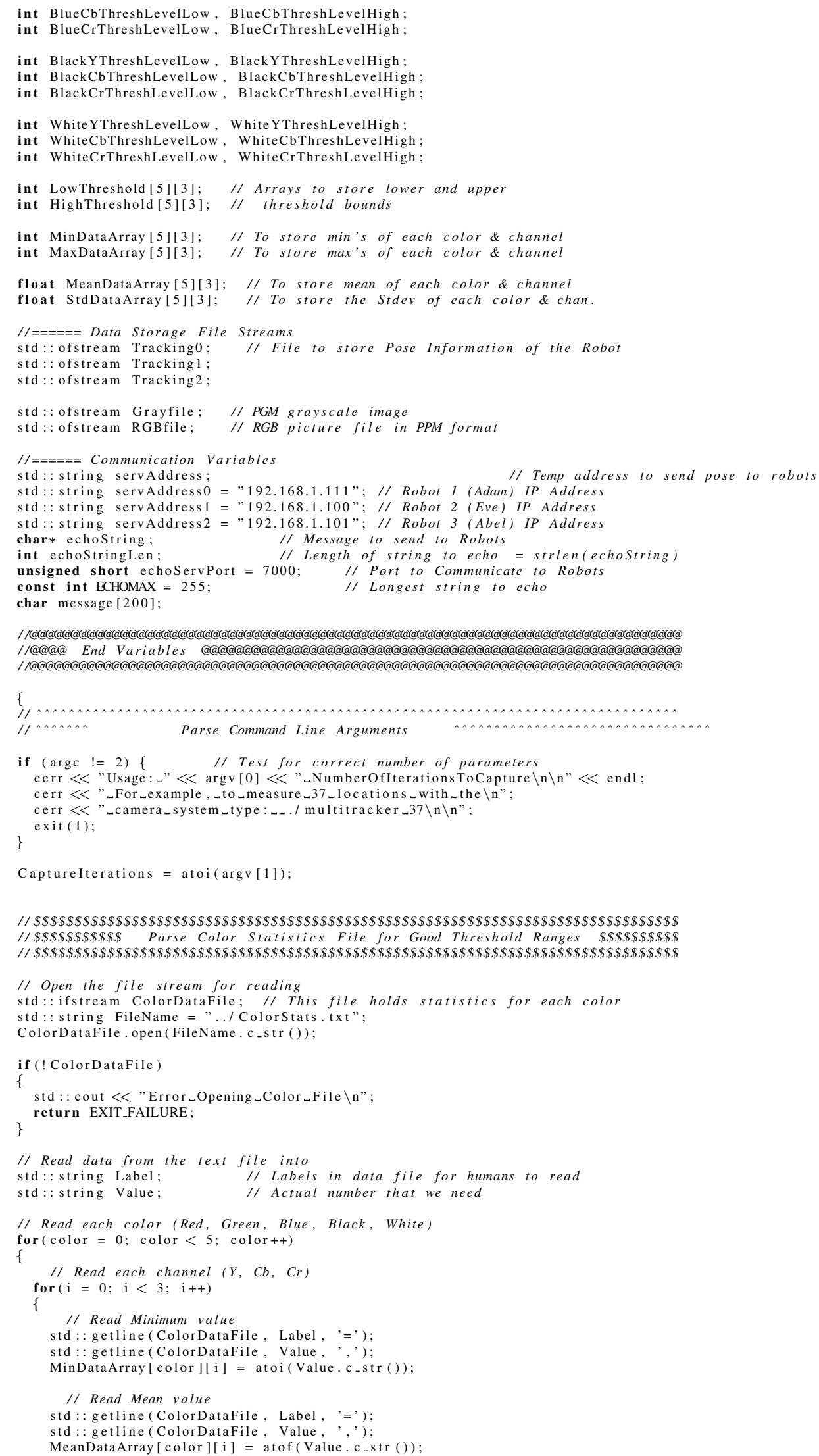




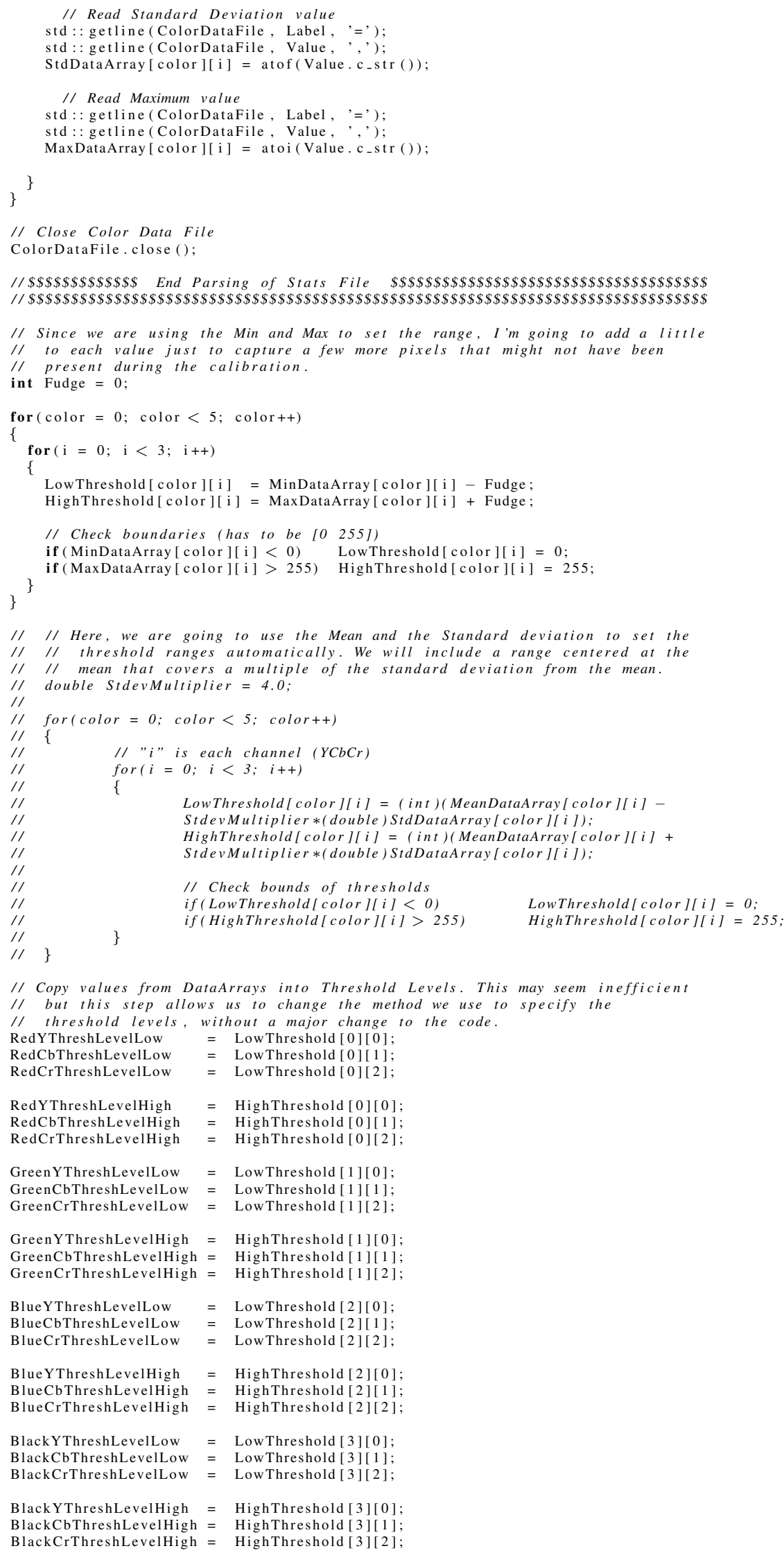




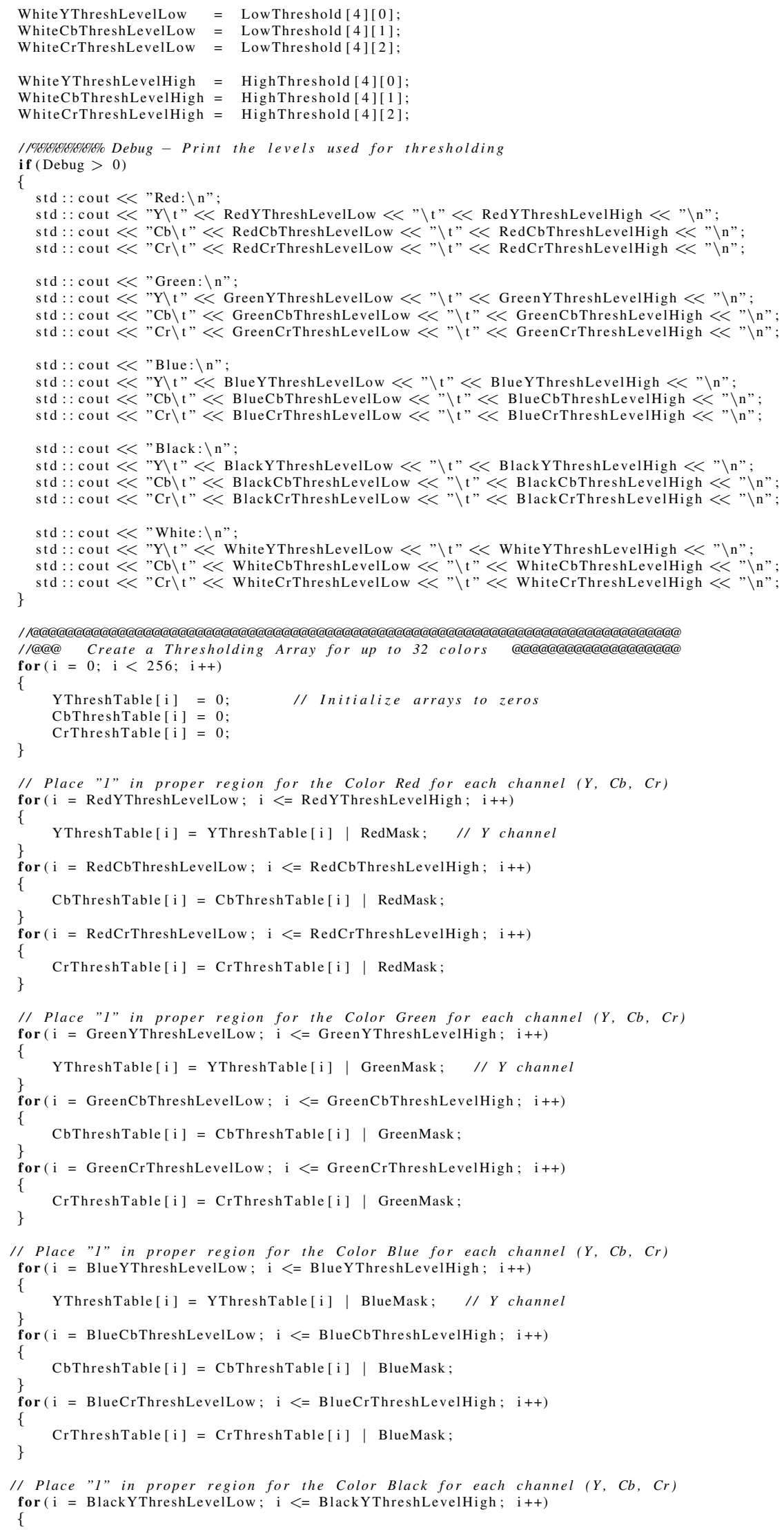




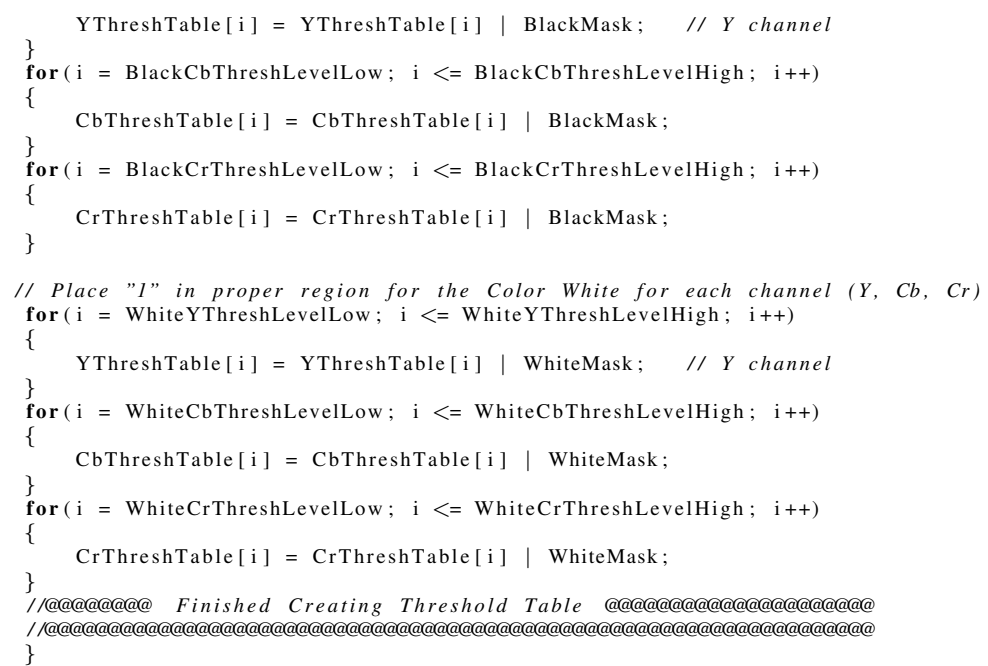


Grab0 . S etB y teOrder (BYTE_ORDER_YUYV);

Grab1. SetBy teOrder (BYTE_ORDER_YUYV);

Grab2 . S et B y te Order (BYTE_ORDER_YUYV);

Grab3 . SetByteOrder (BYTE_ORDER_YUYV);

if $($ Debug $>1)$

\{

std : : cout $<<$ "NewHeight ${ }={ }^{\prime} "<<$ NewHeight $<<" \backslash \mathrm{t} "$

std : : cout $<<$ "NewWidth $\_=-"<<$ NewWidth $<<" \backslash$ t"

std : : cout $<<$ "Padded_Height $=_{-}$" $<<$PaddedHeight $<<$" $\backslash$ t $"$

std : : cout $<<$ "Padded_Width $\_=\_$" $<$PaddedWidth $<<" \backslash$ n";

// Open Text files to store pose information of the robot if ( Debug $>=0)$

\{

Tracking0 .open ("Data/Tracking1011.txt", std : : ios : : out) Tracking 1 . open ("Data/ Tracking2121.txt", std:: ios : : out) Tracking2 . open("Data/Tracking0210.txt", std : : ios : : out);

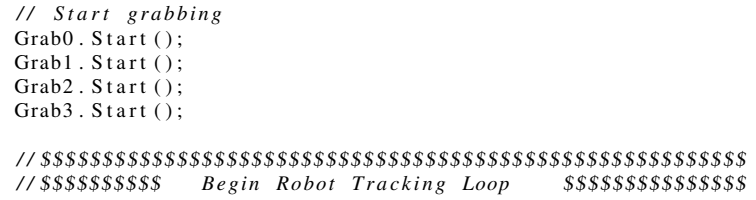




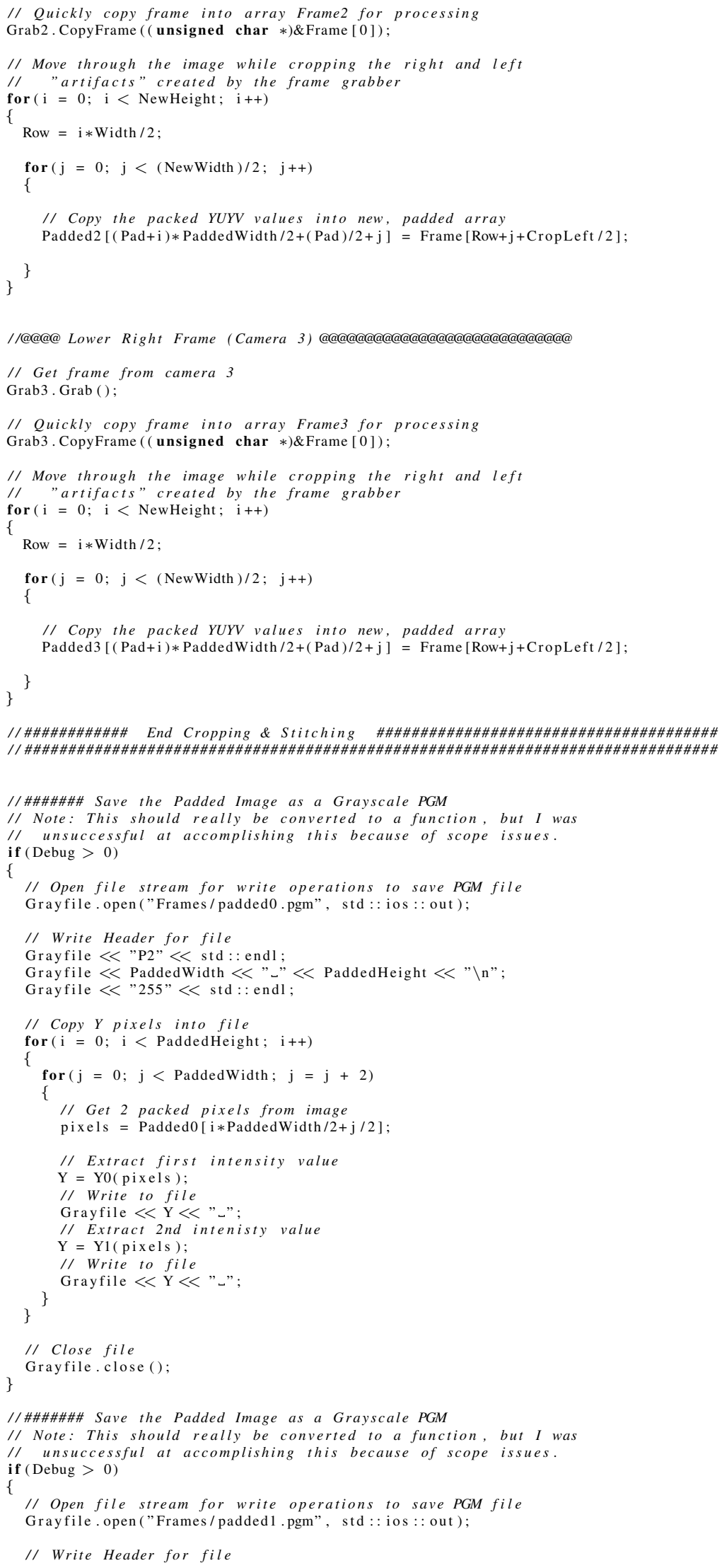




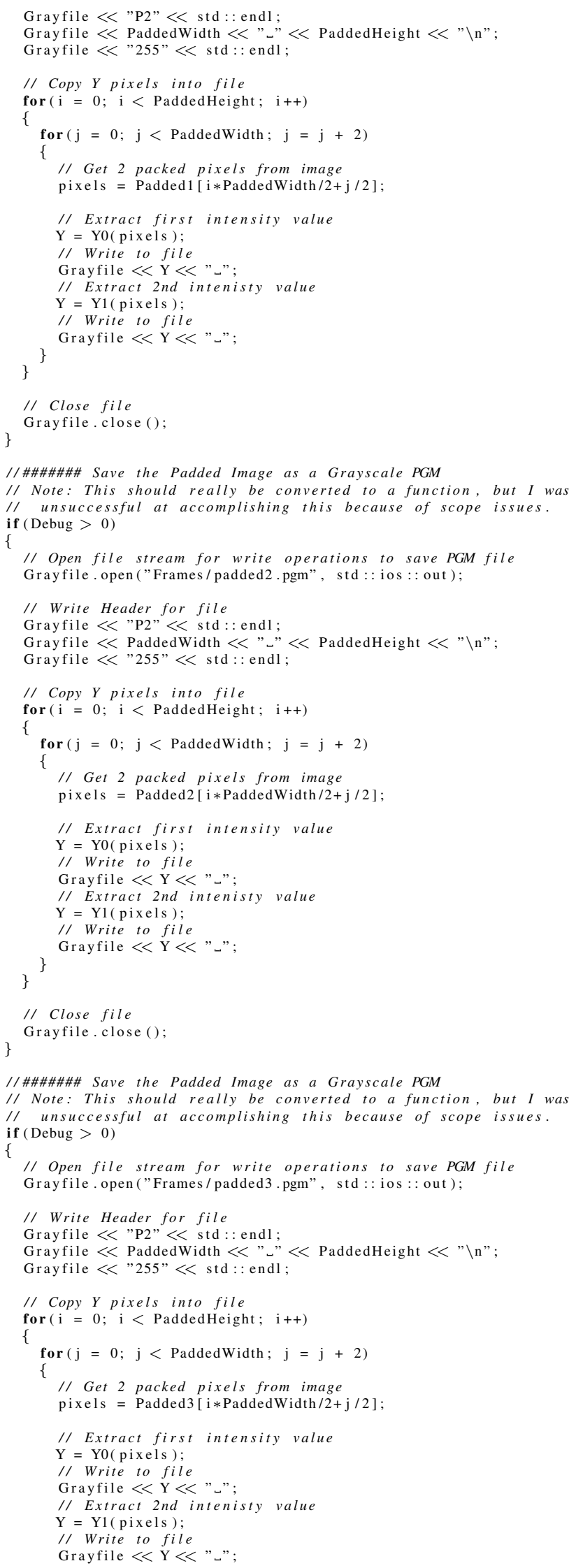




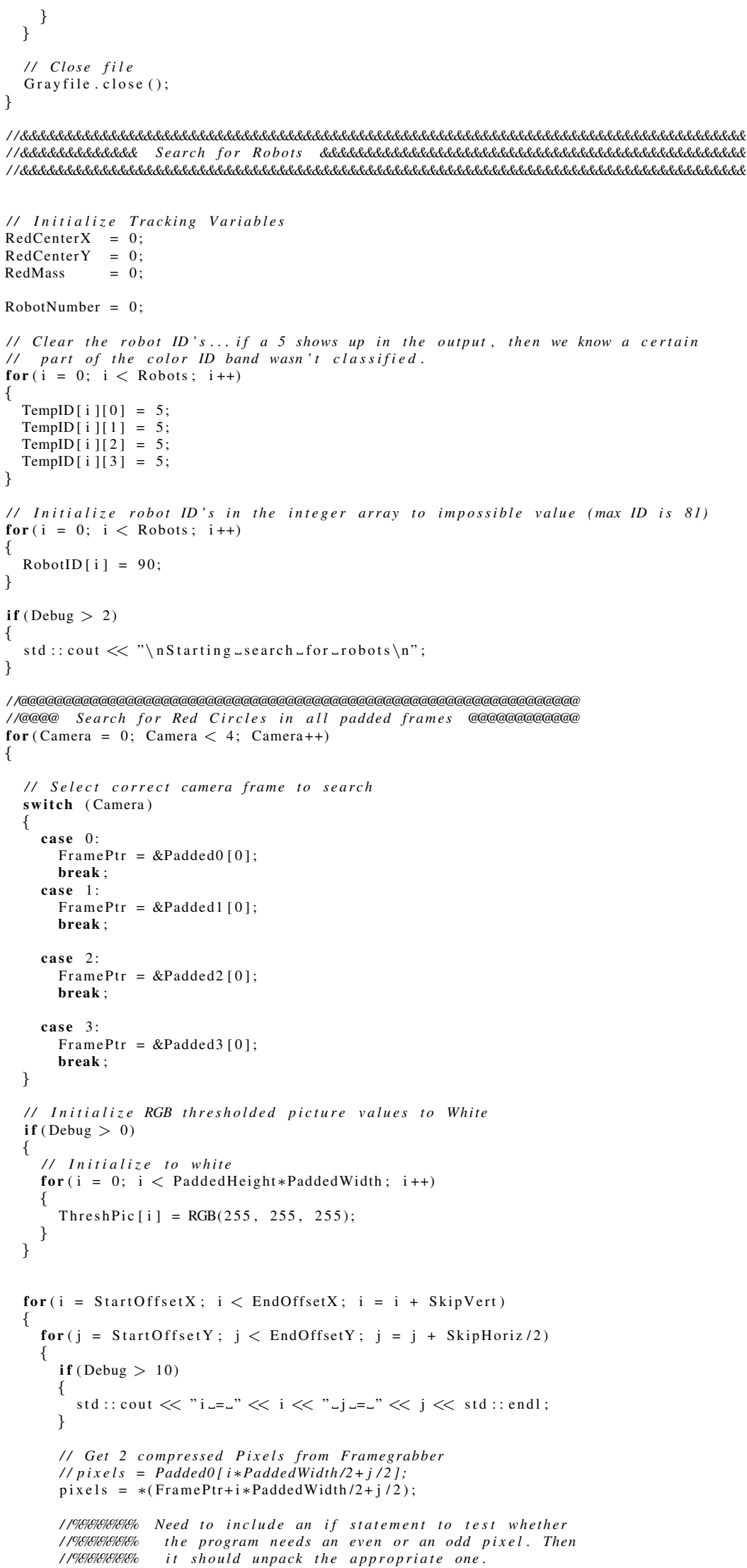




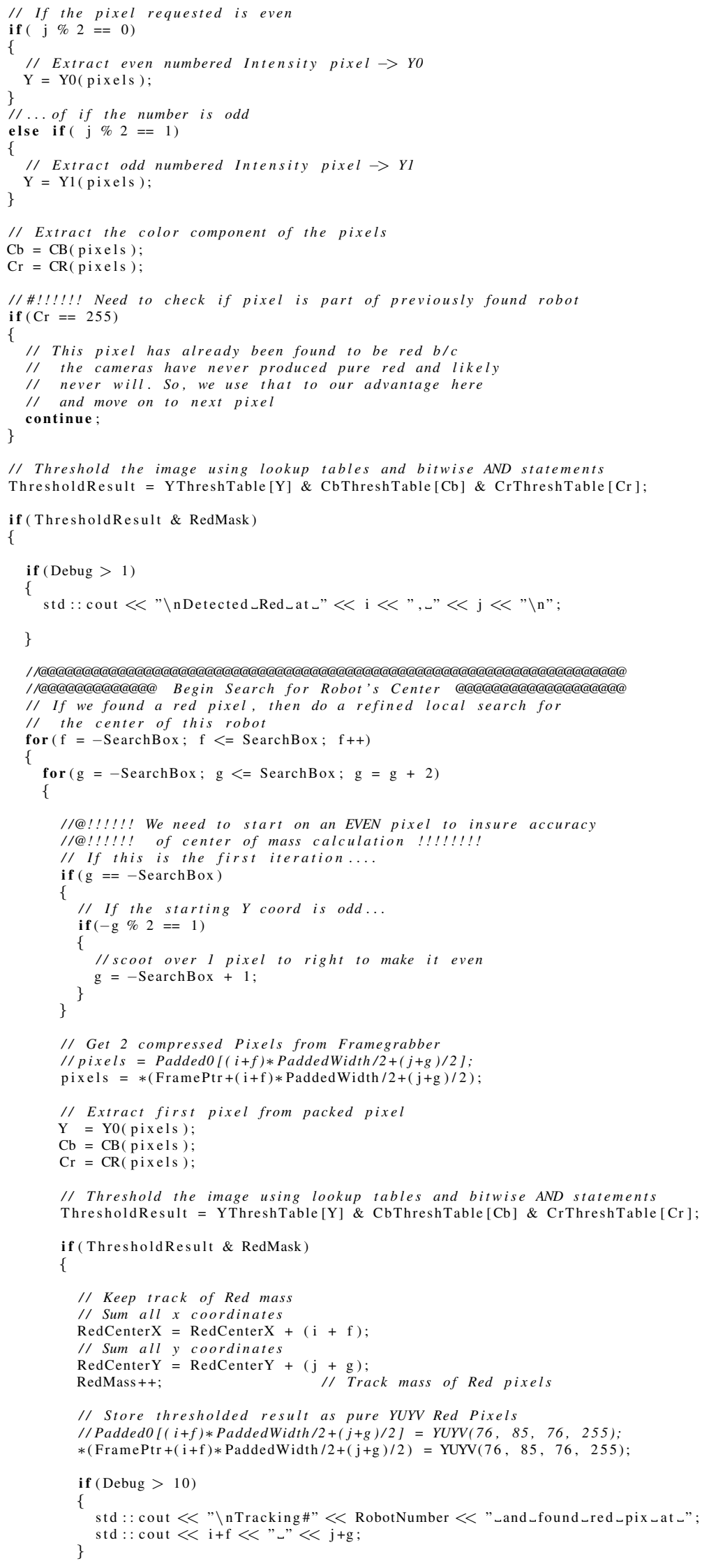




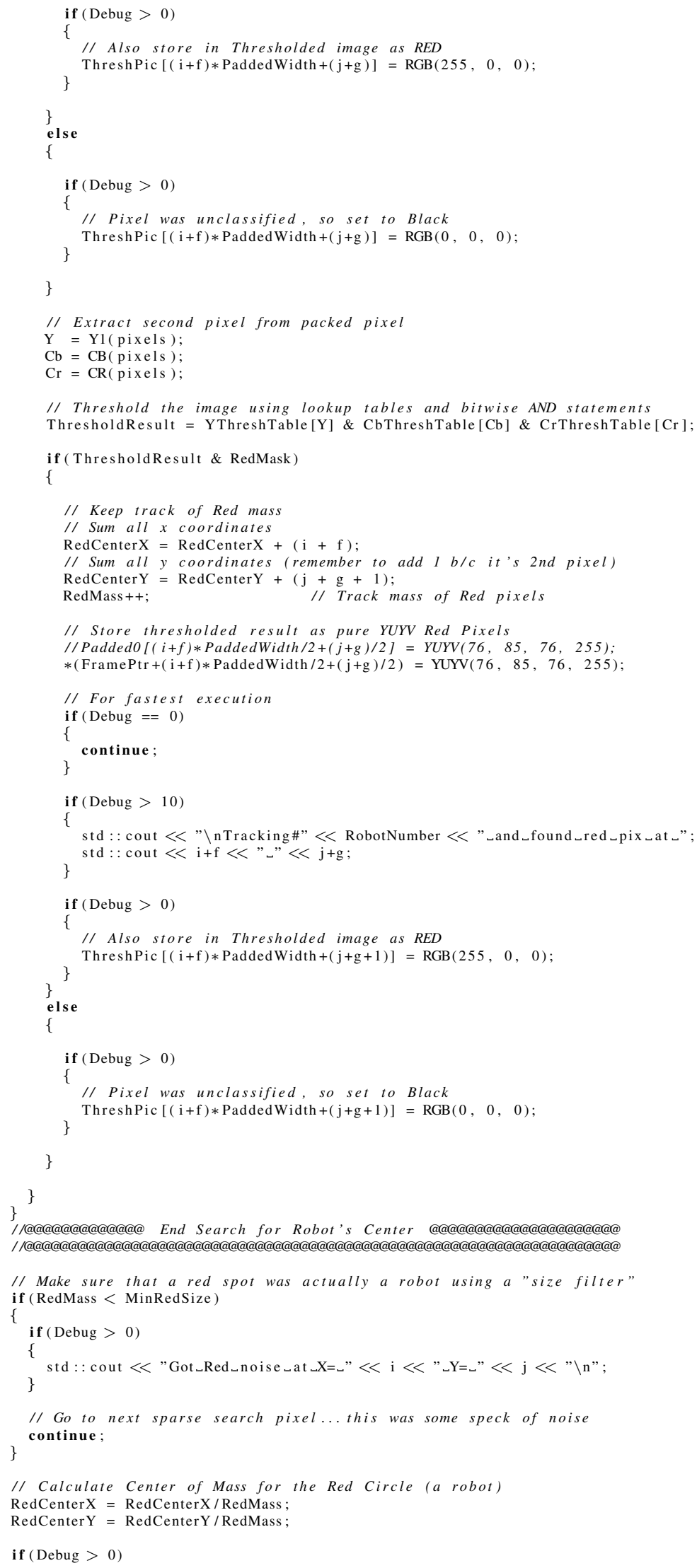




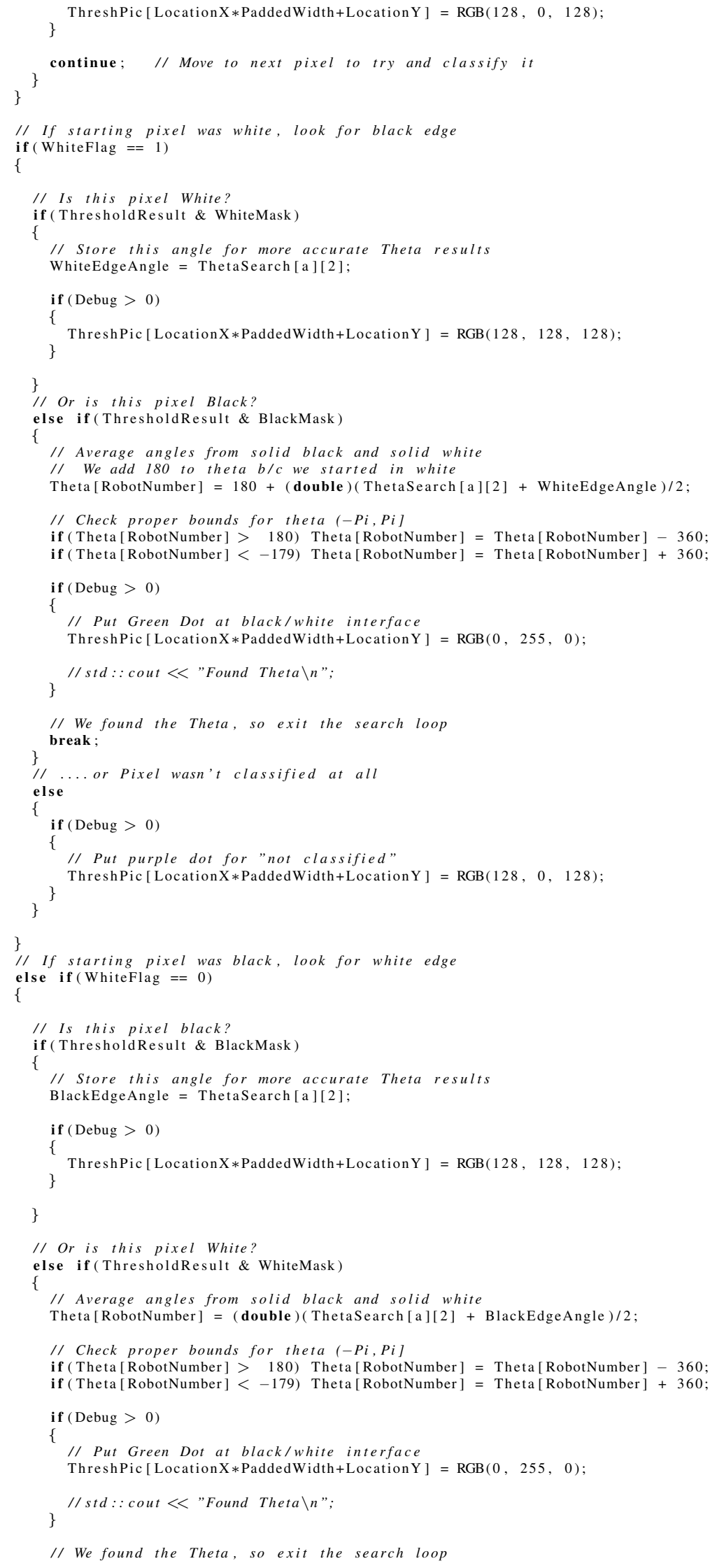




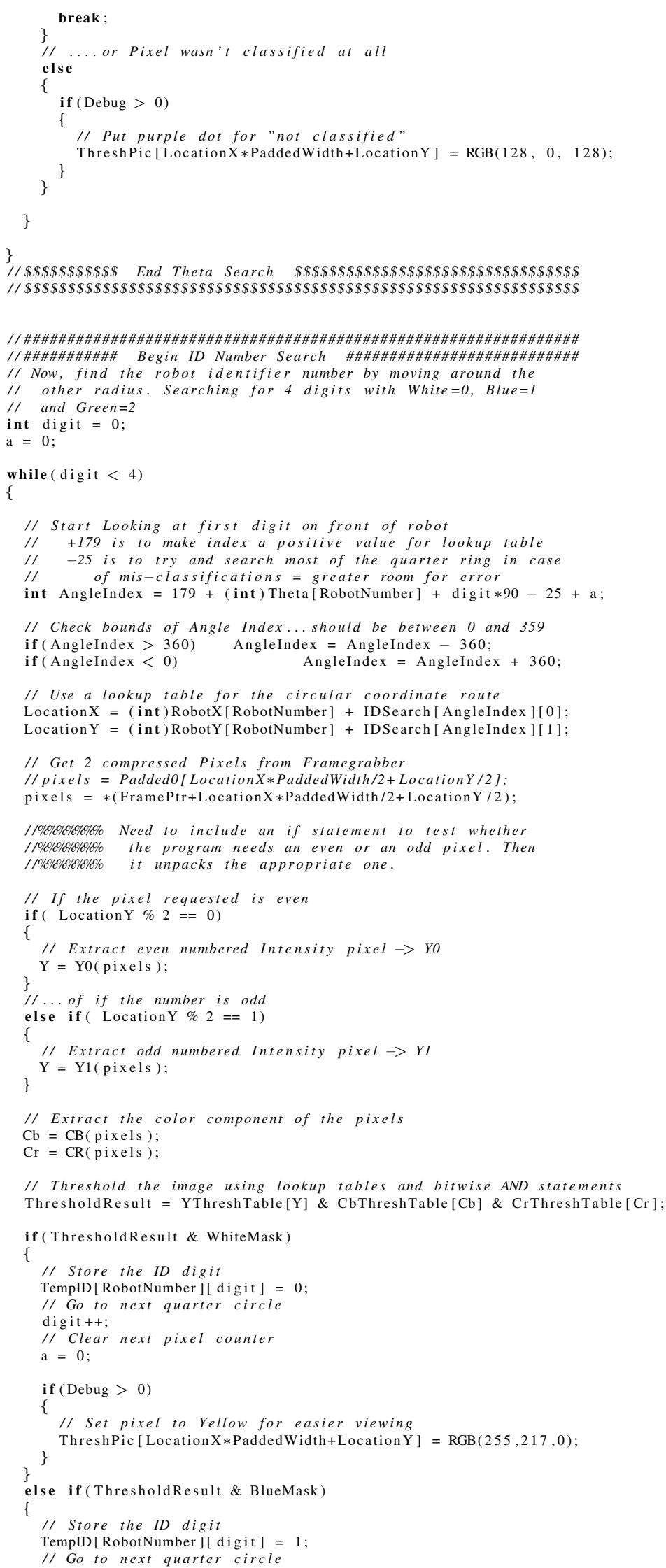




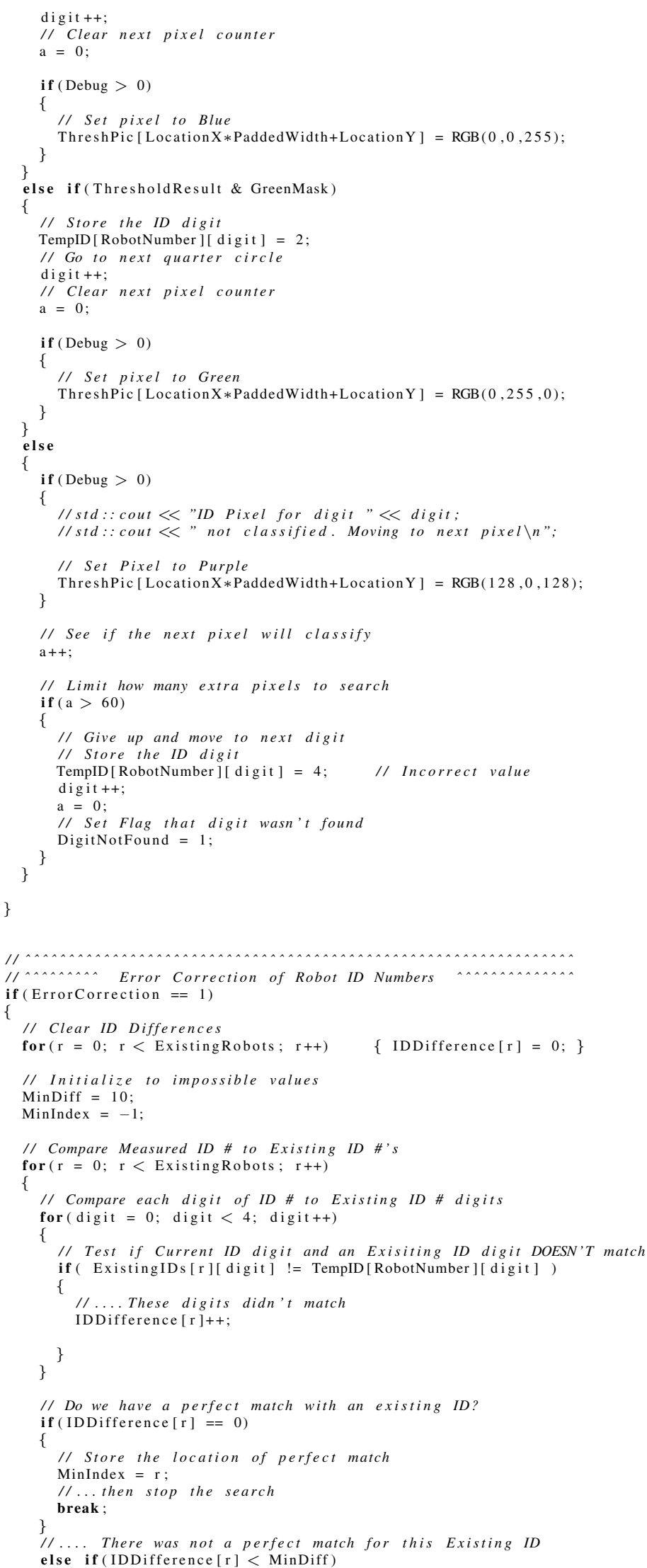




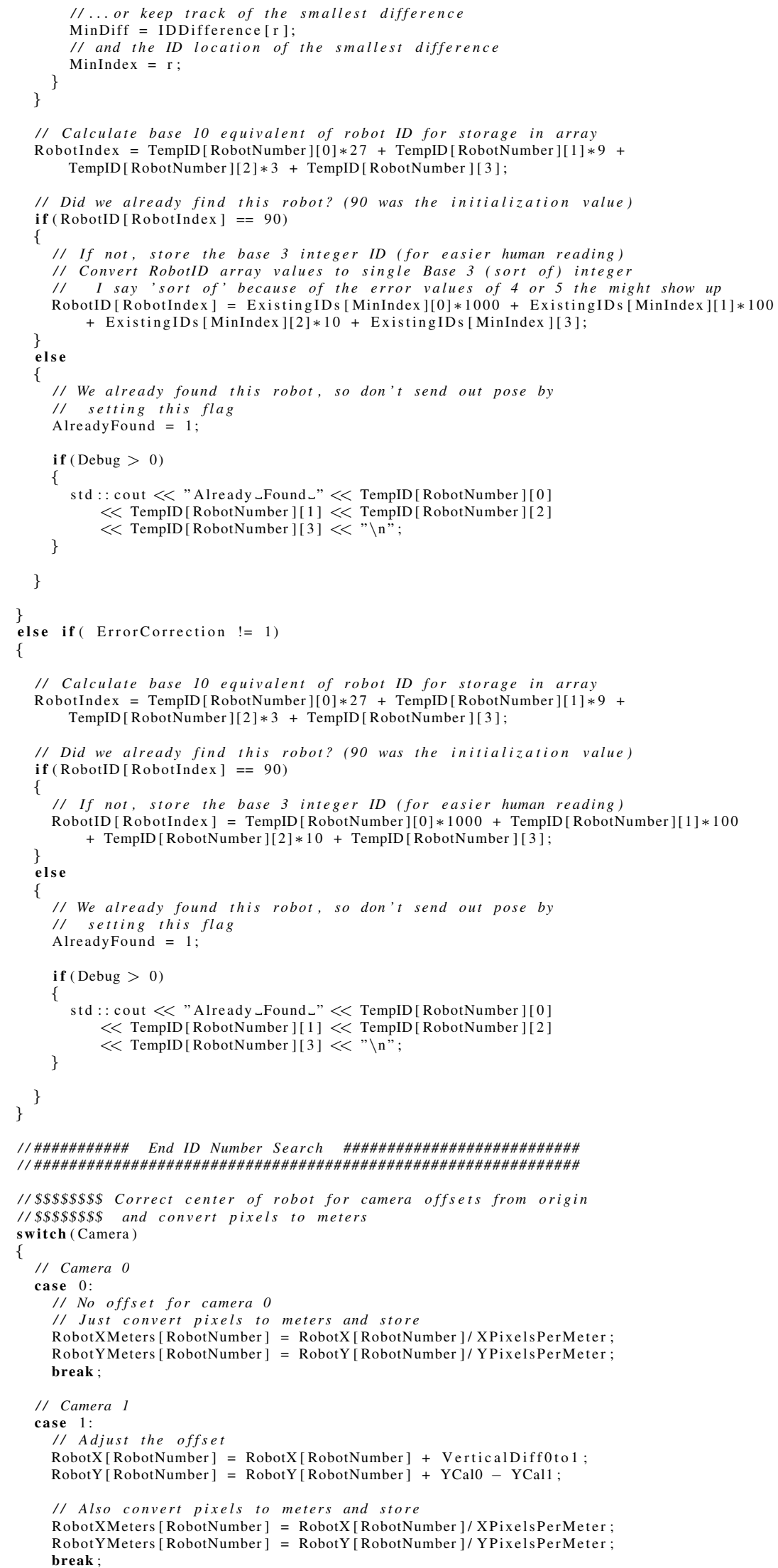




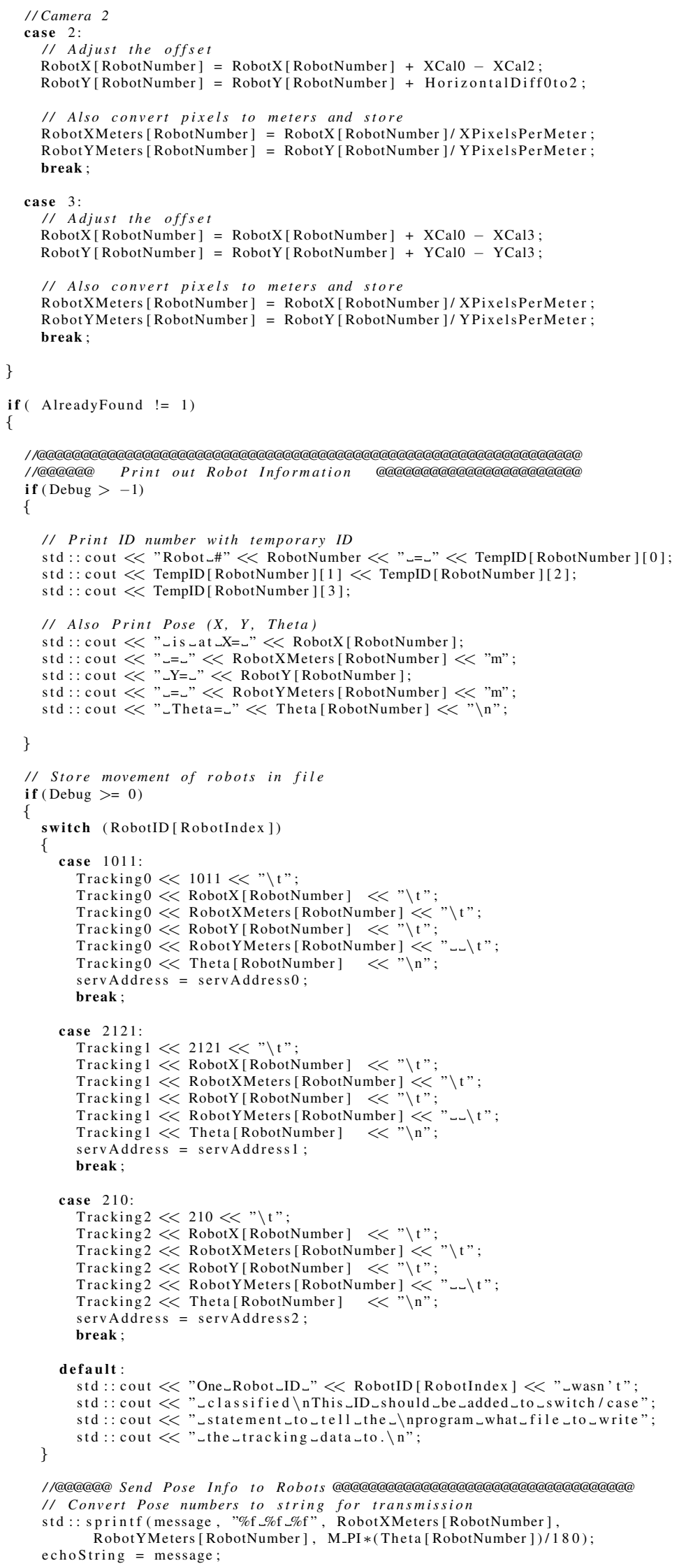




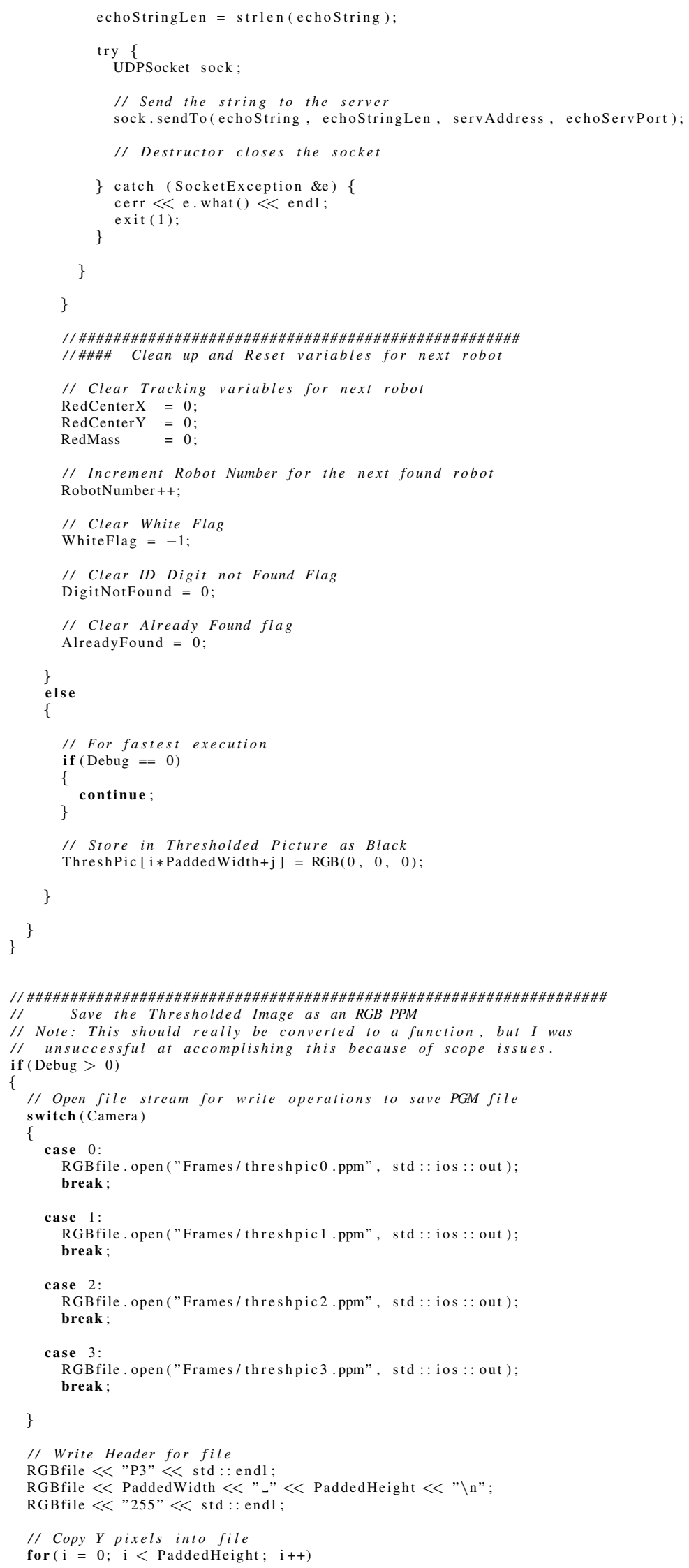




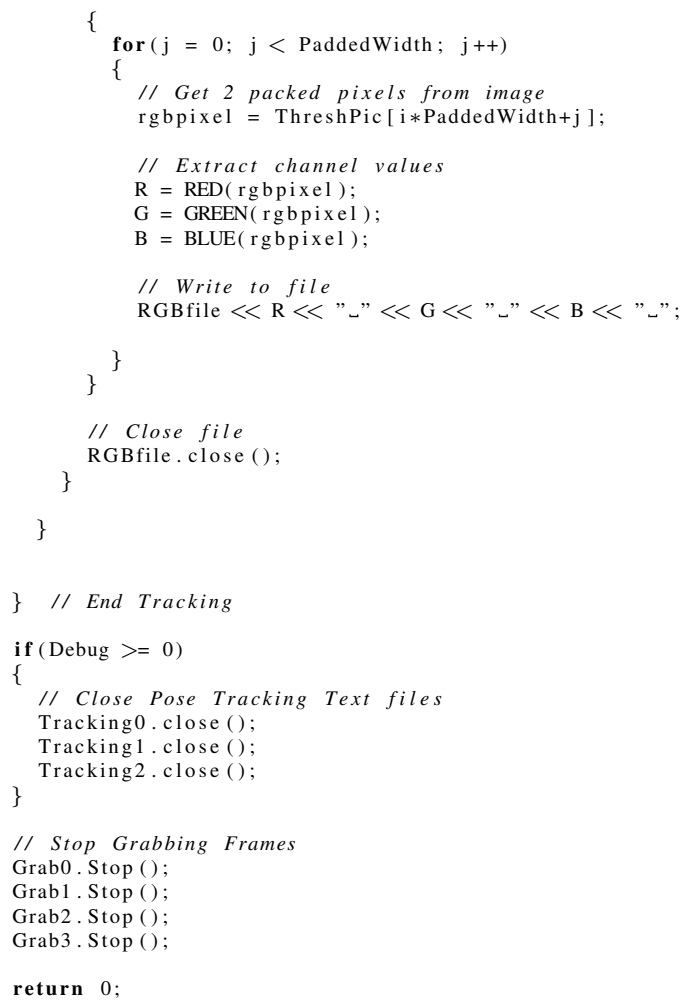


APPENDIX III

\section{Color Threshold Calibration Data}

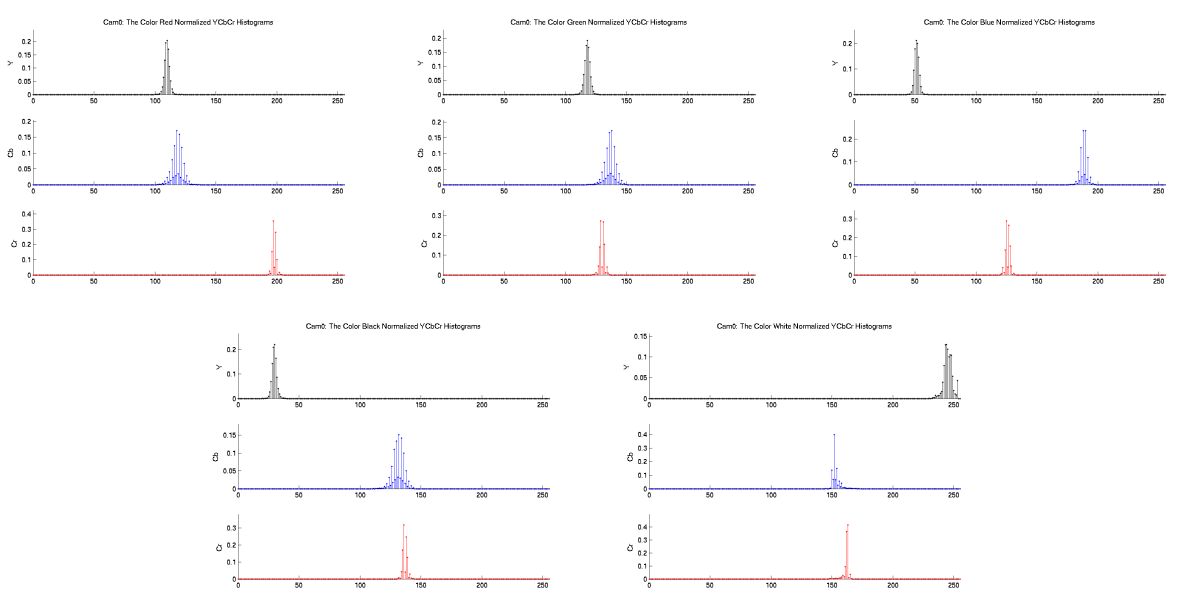

FIGURE 59-Camera 0: From the LEFT: YCbCr Histograms of Red, Green, Blue, Black and White. 


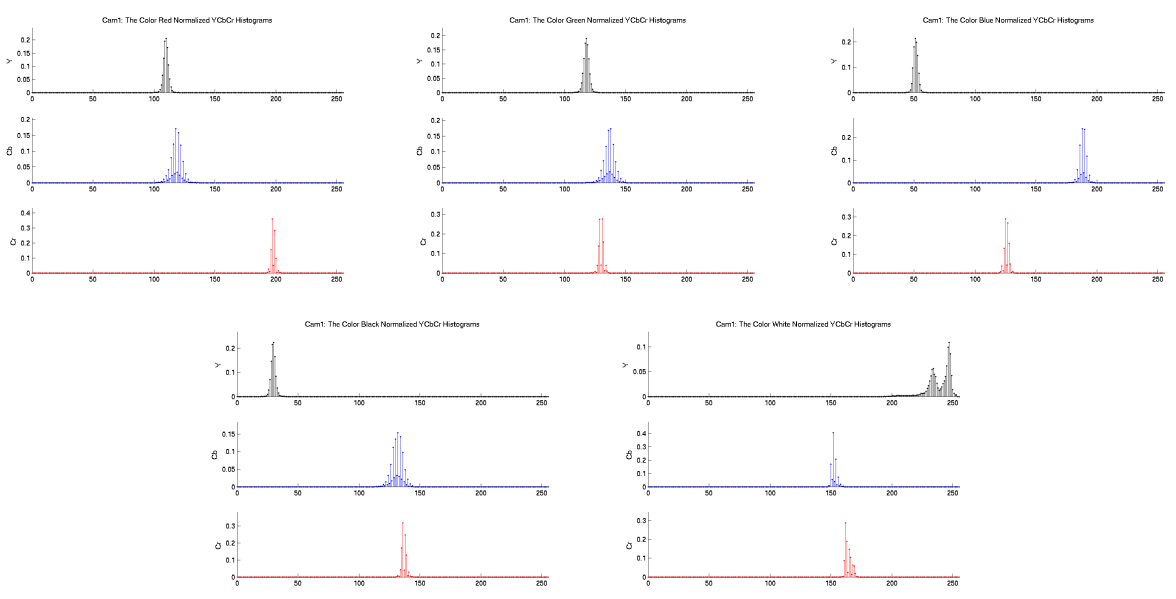

FIGURE 60-Camera 1: From the LEFT: YCbCr Histograms of Red, Green, Blue, Black and White.

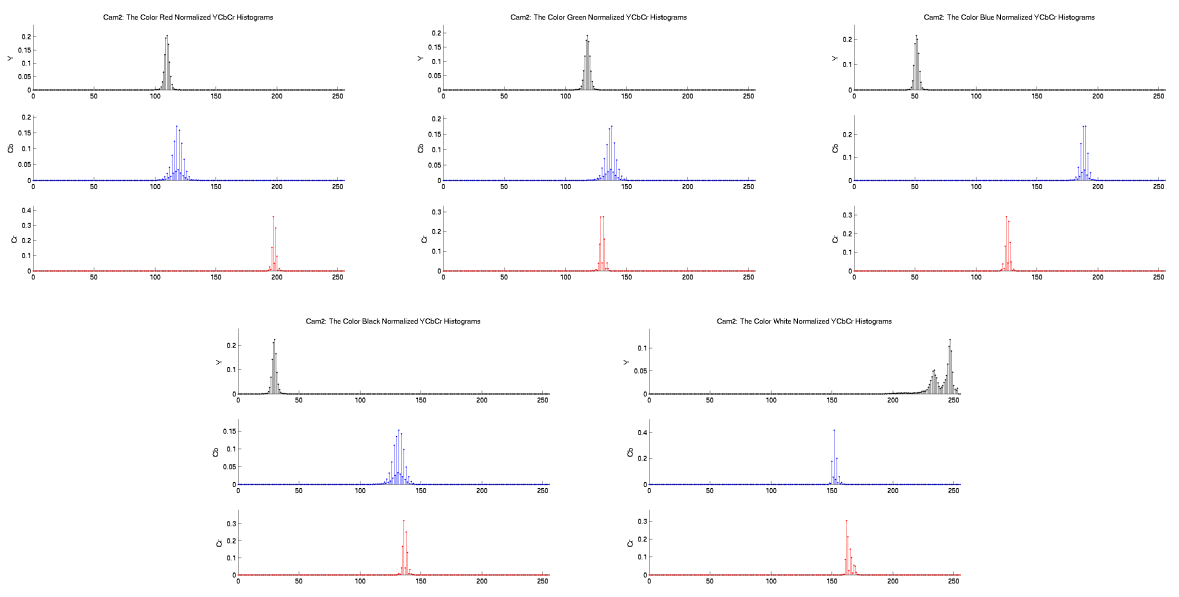

FIGURE 61 -Camera 2: From the LEFT: YCbCr Histograms of Red, Green, Blue, Black and White. 

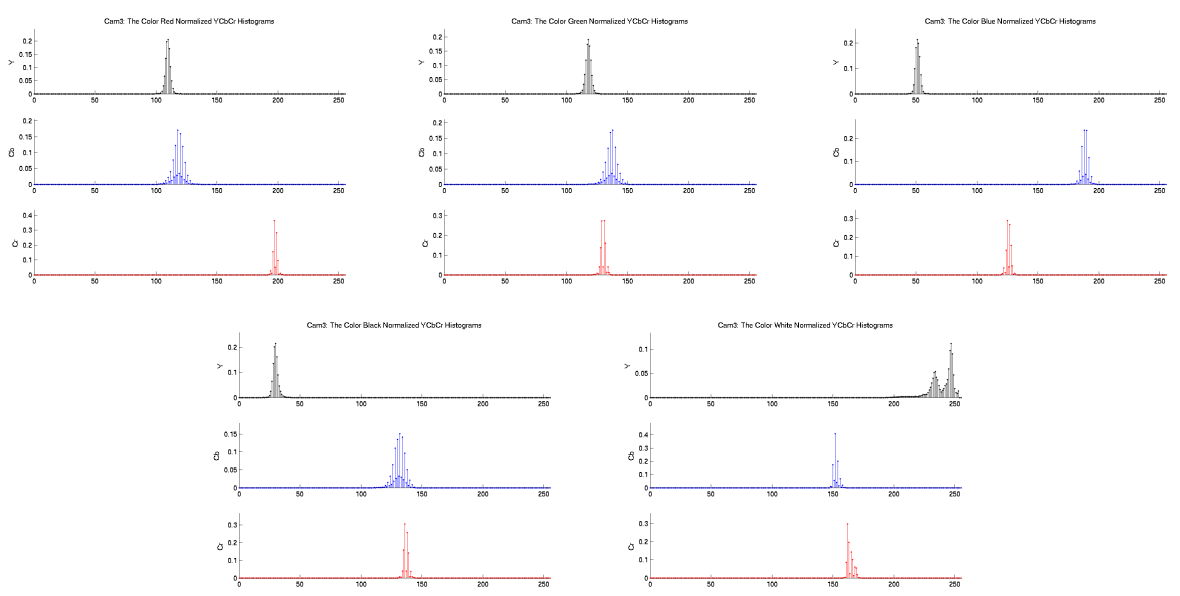

FIGURE 62 -Camera 3: From the LEFT: YCbCr Histograms of Red, Green, Blue, Black and White. 


\section{APPENDIX IV}

\section{Color Threshold Calibration Sampling Program}

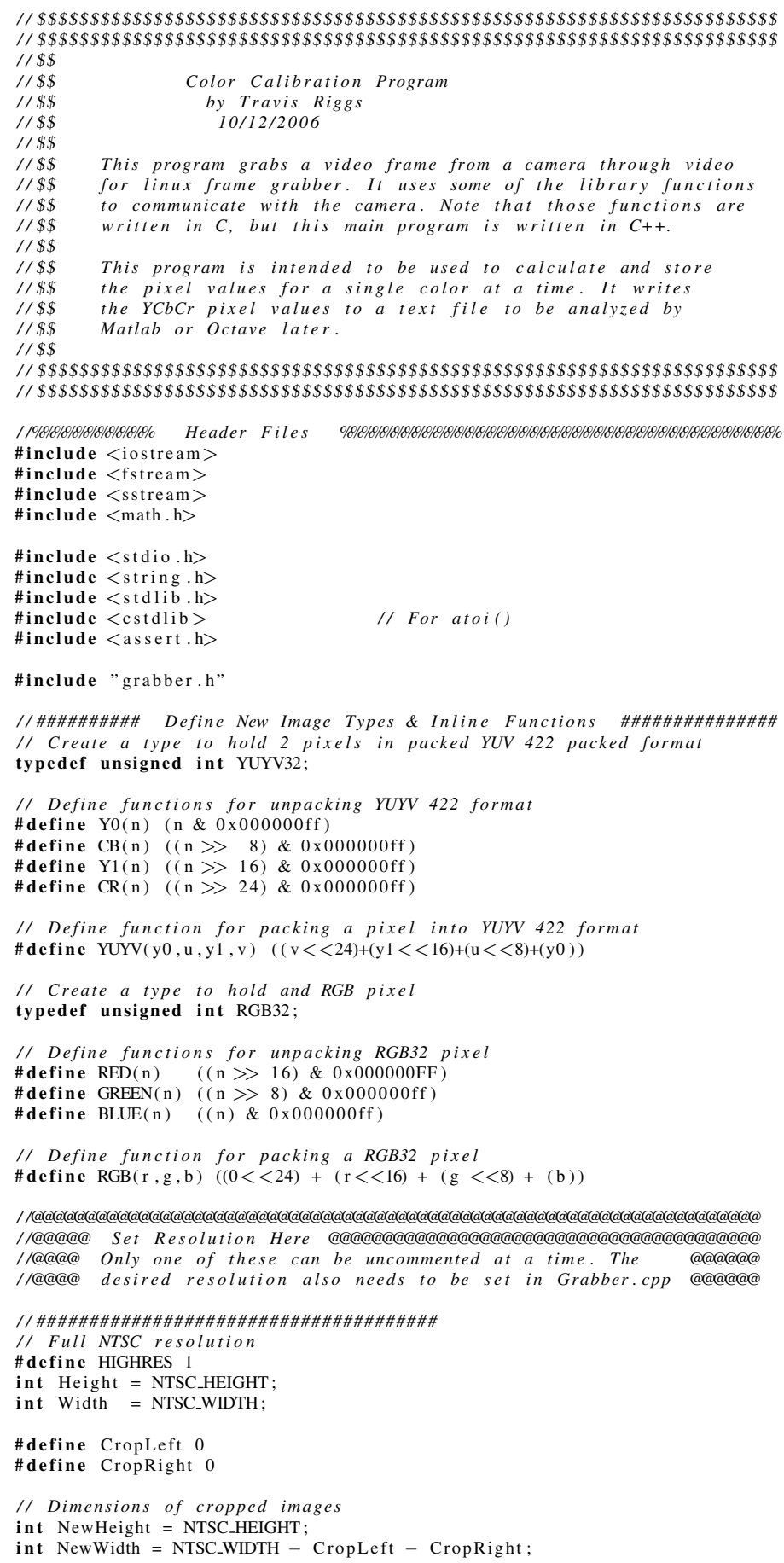


YUYV32 Frame0 [NTSC_HEIGHT *NTSC_WIDTH / 2 ] ;

YUYV32 Frame1 [NTSC_HEIGHT*NTSC_WIDTH/2];

YUYV32 Frame2 [NTSC_HEIGHT *NTSC_WIDTH/2 ]

YUYV32 Frame3 [NTSC_HEIGHT *NTSC_WIDTH/2];

//\#\#\#\# End Full NTSC resolution \#\#\#\#\#\#

// //\#\#\#\#\#\#\#\#\#\#\#\#\#\#\#\#\#\#\#\#\#\#\#\#\#\#\#\#\#\#\#\#\#\#

// // 1/4 NTSC Resolution

// \#define HIGHRES O

$/ /$ int Height $=$ NTSC_HEIGHT/2

I/ int Width $=$ NTSC WIDTH $/ 2$;

$/ /$

// // !!!!!! Need to be EVEN numbers !!!!!!!!

// \#define CropLeft 10

// \#define CropRight 4

//

// // Dimensions of cropped images

$/ /$ int NewHeight $=$ NTSC_HEIGHT/2

// int NewWidth = NTSC_WIDTH/2 - CropLeft - CropRight;

// YUYV32 FrameO [NTSC_HEIGHT*NTSC_WIDTH/8]

// //\#\#\#\# End 1/4 NTSC Resolution \#\#\#\#\#\#\#\#\#\#

int main(int $\operatorname{argc}$, char $* \operatorname{argv}[])$

//@@@@@@@@@@@@@@@@@@@@@@@@@@@@@@@@@@@@@@@a@@@@@@@@@@@a@@@a@@@@@@@@@@@ //1@@@@@@@@ V r ri //@@@@@@@@@@@@@@@@@@@@@@@@@a@@@@@@@@@@@@@@@@@@@@@@@@@@@@@@@@@@@@@@@@@@@@@@@@

int i, j

// Counter variables

nt Row;

char *name;

// Name to store picture files as ppm images

har ans $[10]$;

// Input from user

Yval = 0

// Actual pixel values in $\mathrm{YCbCr}$ (YUV) space

Cbval $=0$

int Crval $=0$;

YUYV32 pixels; // Structure to hold packed YUYV 422 pixels $(2$ pixels)

// Instantiate Grabber objects to grab frames

Grabber Grab0; $\quad$ // For camera

Grabber Grab1; $\quad$ // For camera I

Grabber Grab2; $\quad$ // For camera 2

Grabber Grab3; $\quad$ // For camera 3

std:: ofstream mycolorfile; $\quad / /$ File to store color data for anal in Matlab std : : ofstream grayfile;

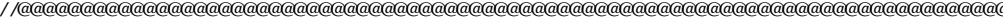

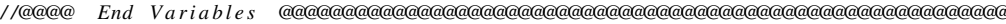
//@@@@@@@@@@@@@@@@@@@@@@@@@@@@@@@@@@@@@@@@@@@@@@@@@@@@@@@@@@@@@@@@@@@@@@

/l\#\#\#\#\#\#\#\#\#\#\#\#\#\#\#\#\#\#\#\#\#\#\#\#\#\#\#\#\#\#\#\#\#\#\#\#\#\#\#\#\#\#\#\#\#\#\#\#\#\#\#\#\#\#\#\#\#\#\#\#\#\#\#\#\#\#\#\#\#\#

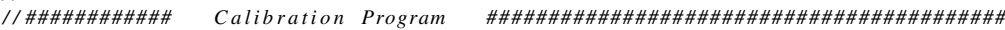

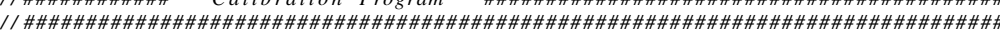

// Setup the Frame Grabber and Cameras

// Open video device with YUV 422 format

Grab0. Init (CF_422, "/dev/video0", true);

Grab1. Init (CF_422, "/dev/video1", true);

Grab2. Init (CF-422, "/dev/video2", true);

Grab3. Init (CF_422, "/ dev/video3", true);

// Set channel of multiplexor for input

Grab0. SetChannel ("Composite0");

Grab1. SetChannel ("Composite0");

Grab2. SetChannel ("Composite0");

Grab3. SetChannel ("Composite0")

// Video signal configure

Grab0. SetVideoSignal ("ntsc")

Grab1. SetVideoSignal ("ntsc")

Grab2. SetVideoSignal ("ntsc");

Grab3. SetVideoSignal ("ntsc");

Grab0 . SetByteOrder (BYTE_ORDER_YUYV); Grab1 . SetByteOrder (BYTE_ORDER_YUYV);

Grab2. SetB yteOrder (BYTE_ORDER_YUYV);

Grab3. SetByteOrder (BYTE_ORDER_YUYV);

// Start grabbing

Grab0. Start ();

Grab1. Start ();

Grab2. Start ();

Grab3. Start (),

$1 /$ Inform User of Calibration Process 


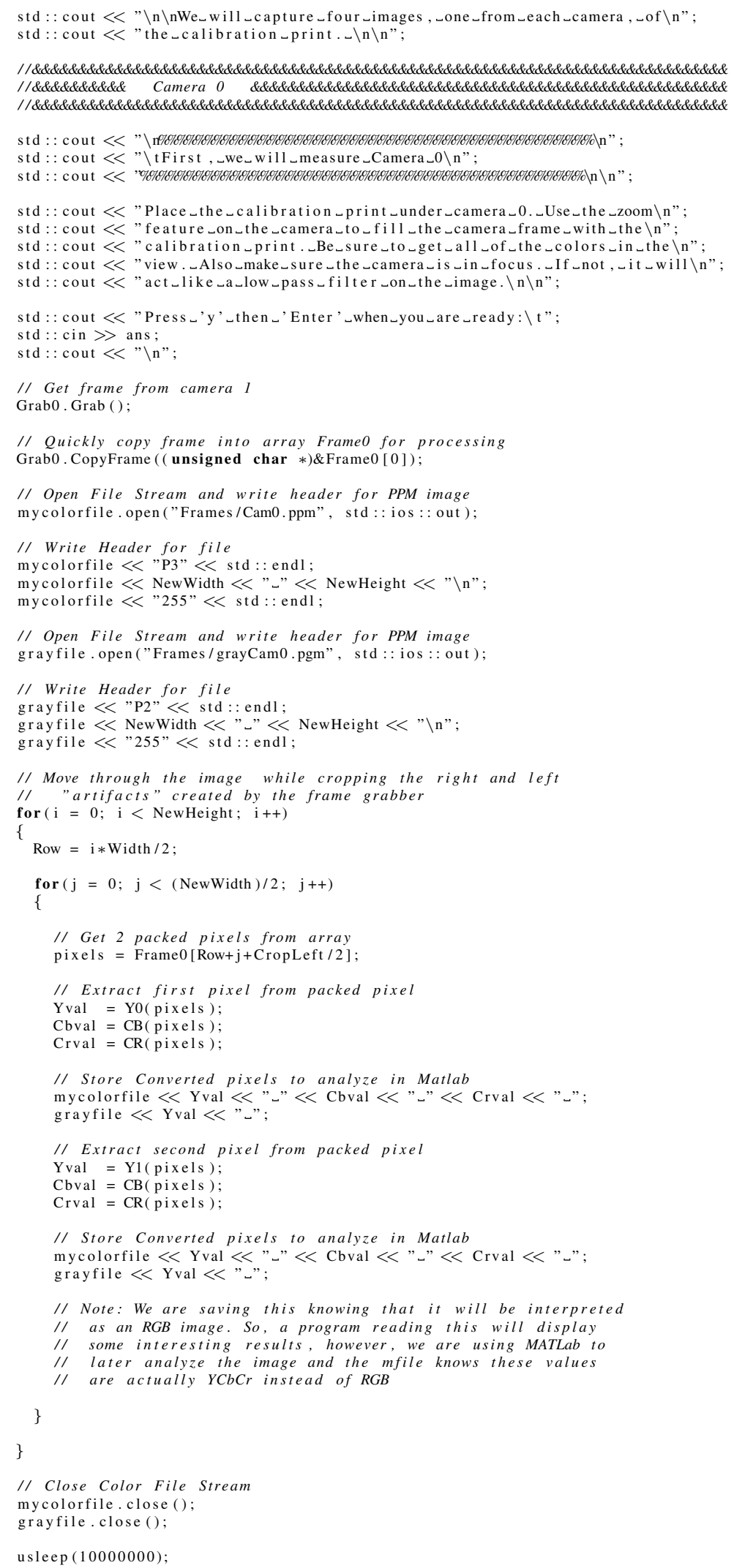

//\&\&\&\&\&\&\&\&\&\&\&\&\&\&\&\&\&\&\&\&\&\&\&\&\&\&\&\&\&\&\&\&\&\&\&\&\&\&\&\&\&\&\&\&\&\&\&\&\&\&\&\&\&\&\&\&\&\&\&\&\&\&\&\&\&\&\&\&\&\&\&\&\&\&\& //\&\&\&\&\&\&\&\&\& Camera 1 \&\&\&\&\&\&\&\&\&\&\&\&\&\&\&\&\&\&\&\&\&\&\&\&\&\&\&\&\&\&\&\&\&\&\&\&\&\&\&\&\&\& //\&\&\&\&\&\&\&\&\&\&\&\&\&\&\&\&\&\&\&\&\&\&\&\&\&\&\&\&\&\&\&\&\&\&\&\&\&\&\&\&\&\&\&\&\&\&\&\&\&\&\&\&\&\&\&\&\&\&\&\&\&\&\&\&\&\&\&\&\&\&\&\&\&\&\&\& 


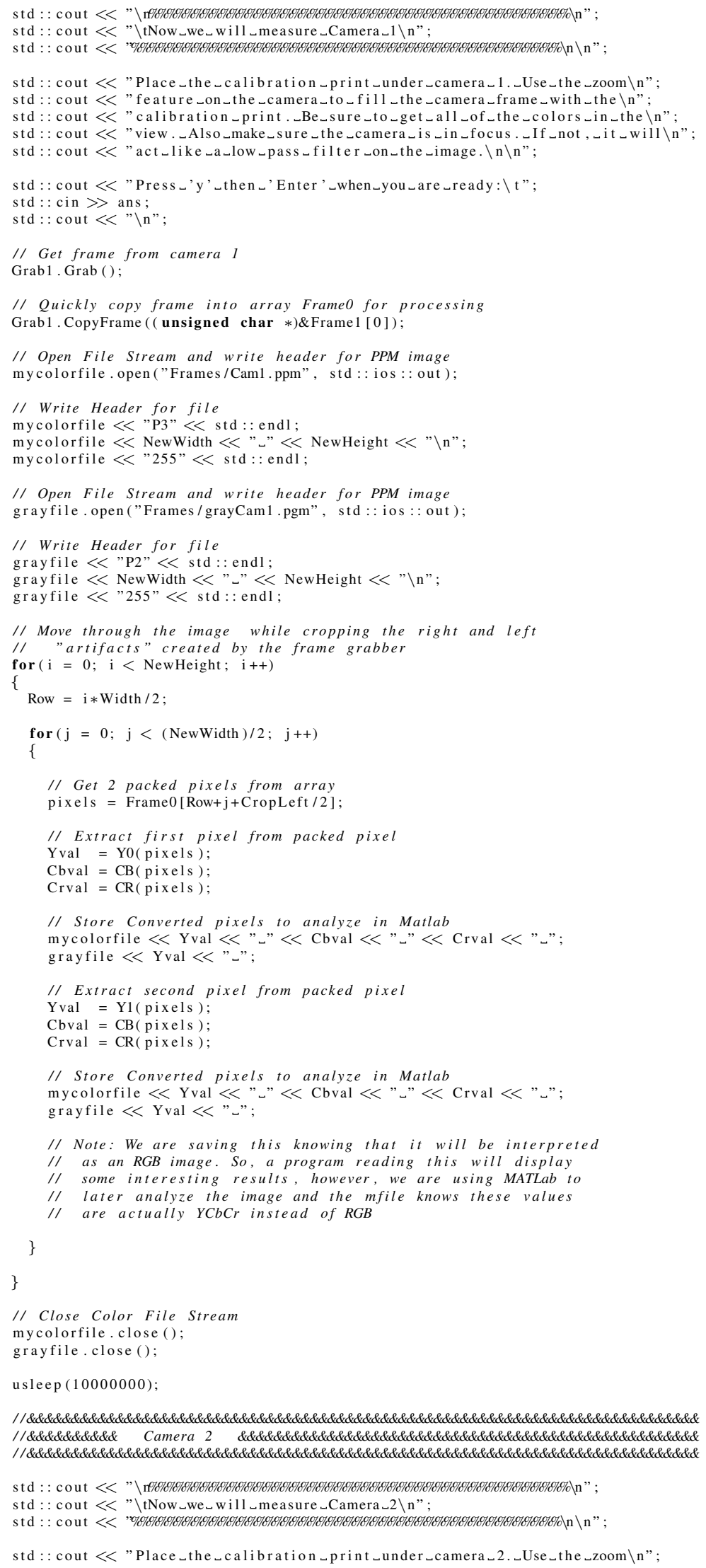




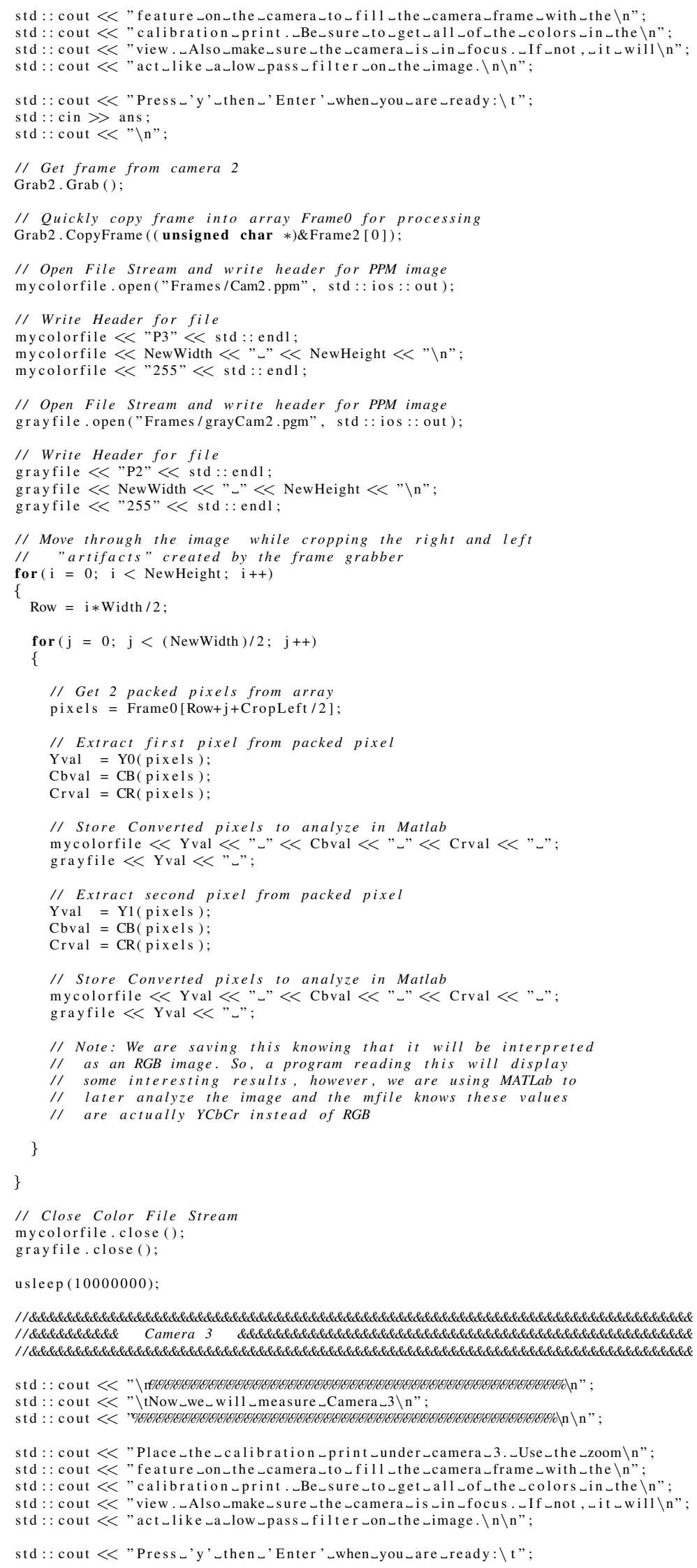




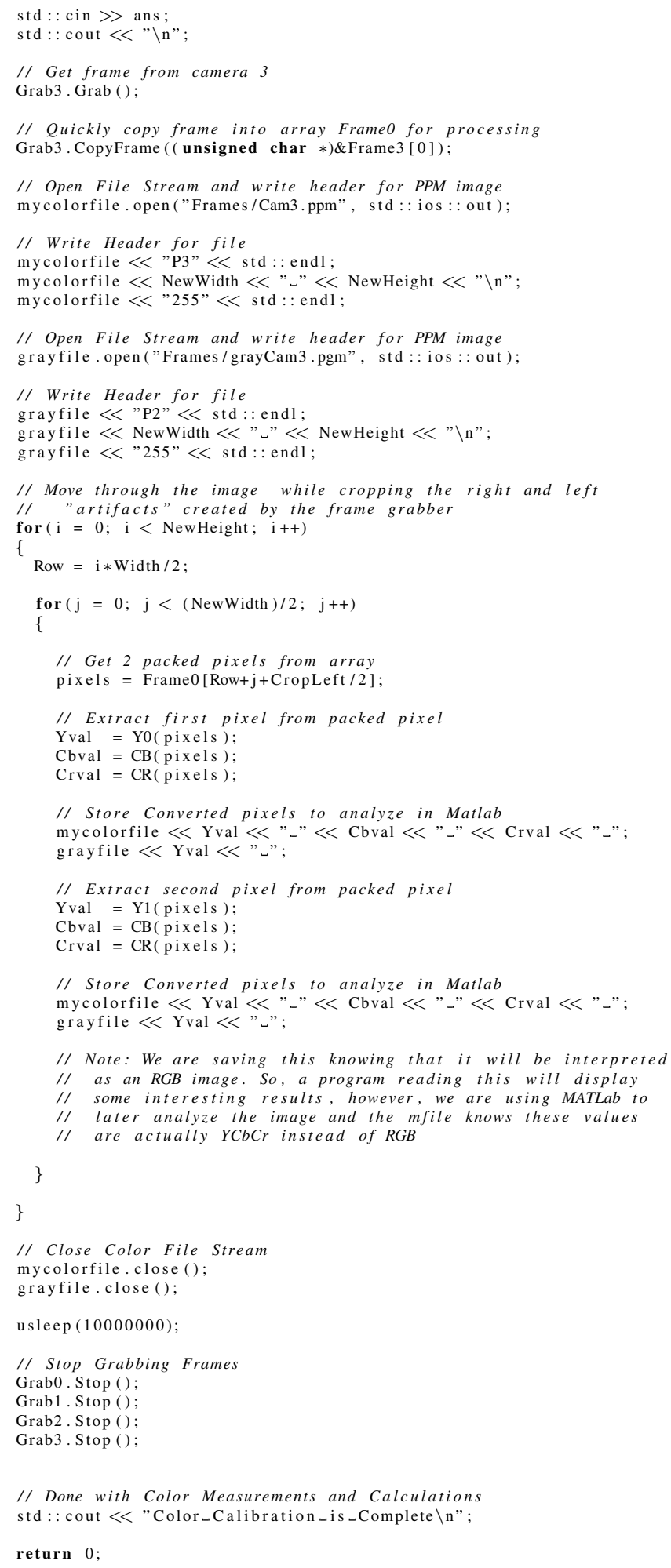




\section{APPENDIX V \\ Color Threshold Calibration Program}

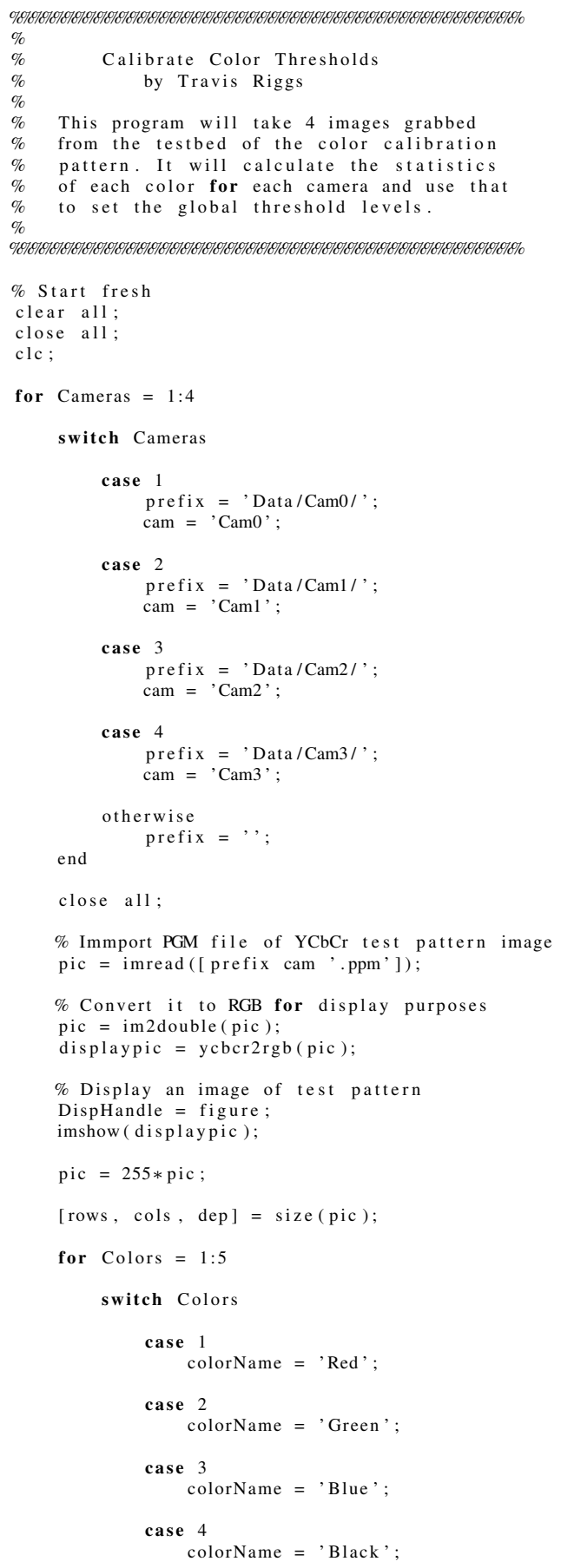




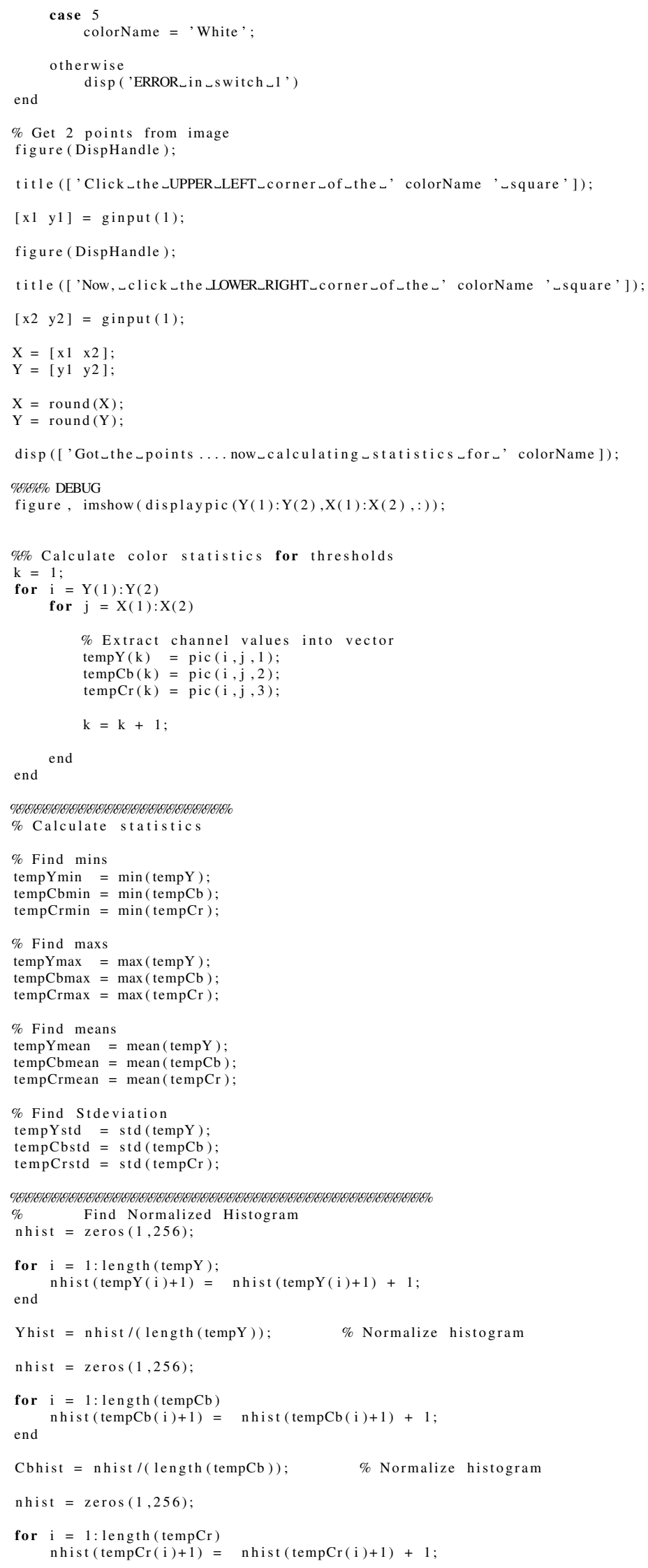




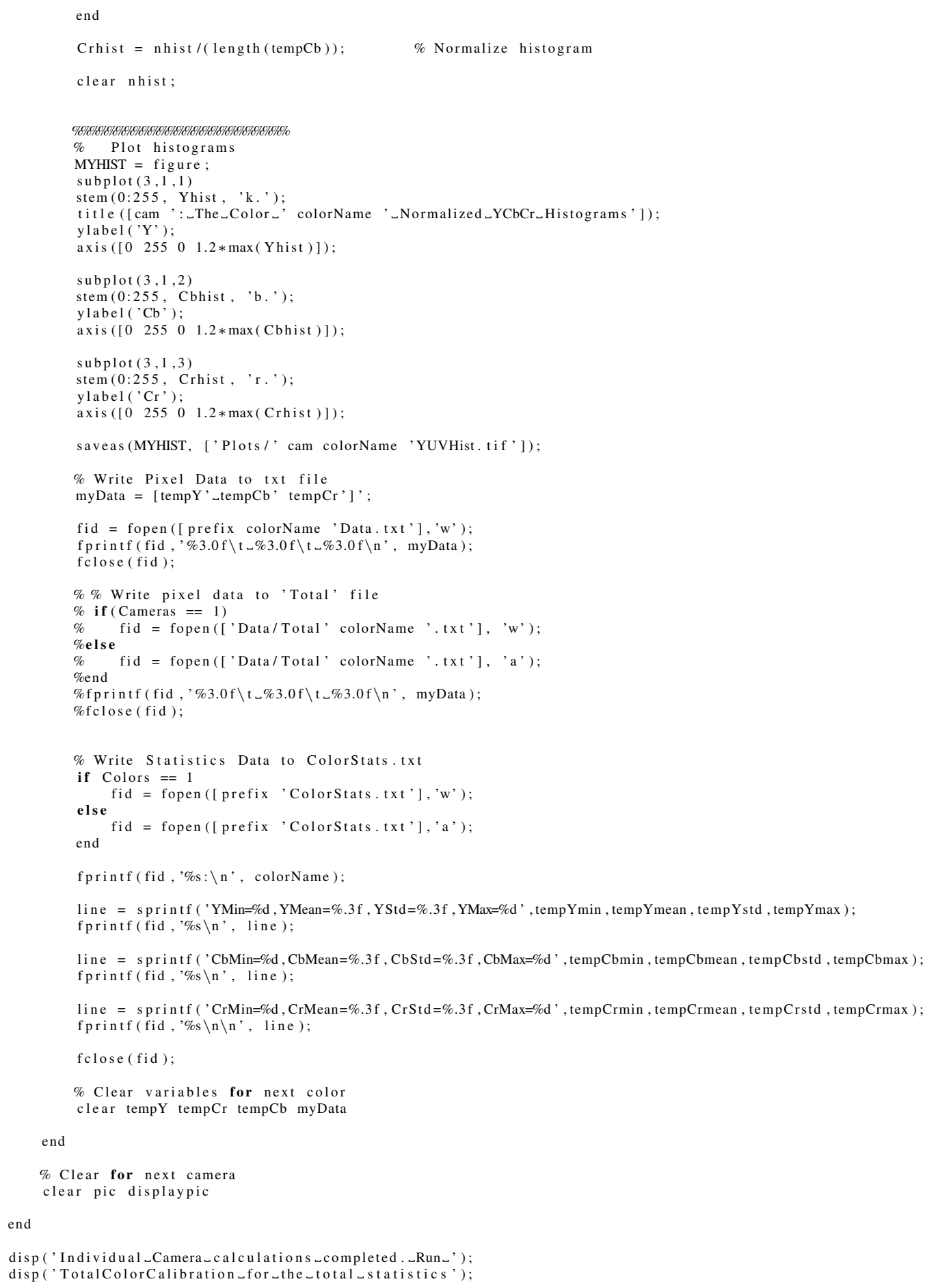




\section{APPENDIX VI}

\section{Kernel Modification Files}

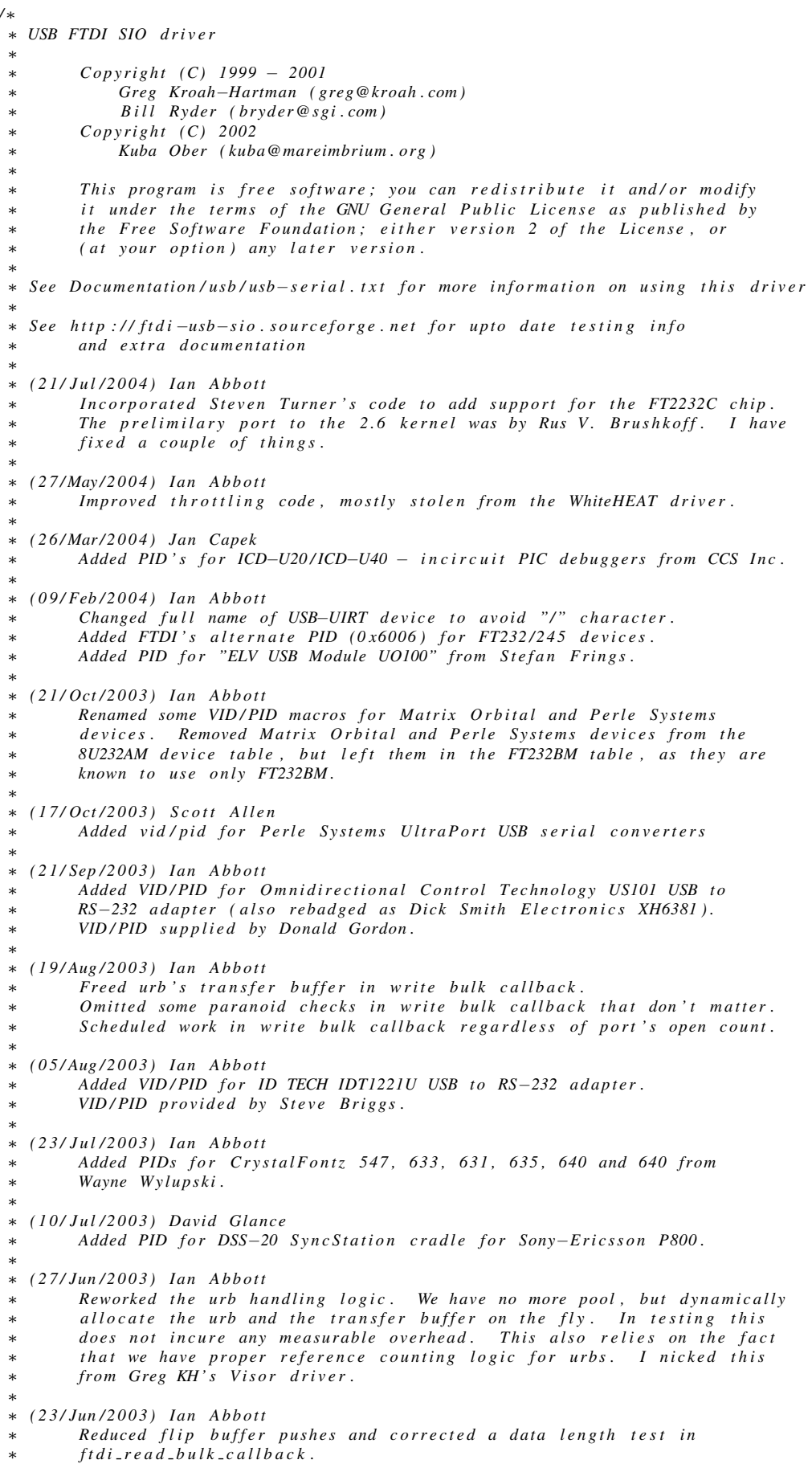




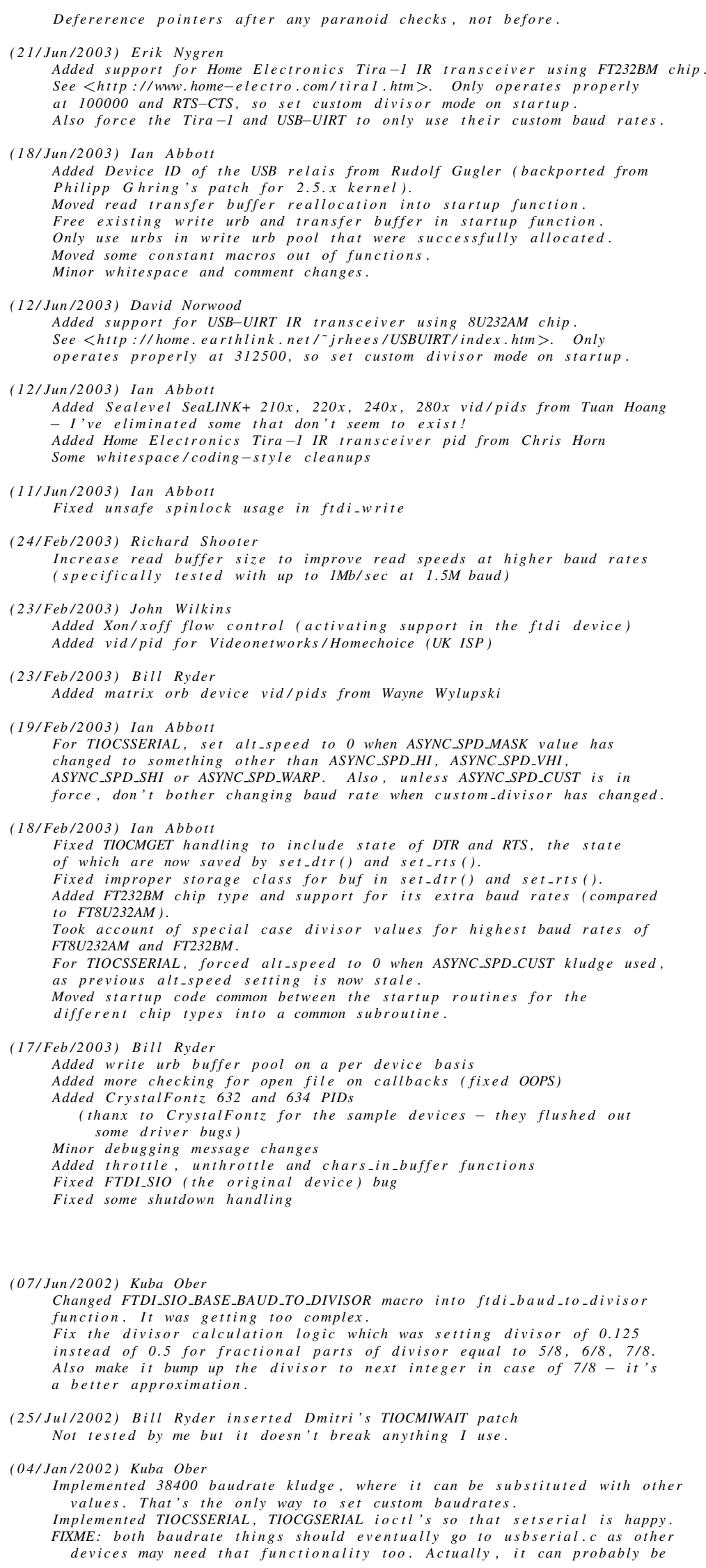


\#include $<$ linux/serial.h $>$

\#include "usb-serial.h"

\#include "ftdi_sio.h"

$/ *$

* Version Information

\#define DRIVER VERSION "v1 4.2,

\#define DRIVER_AUTHOR "Greg_Kroah-Hartman_<greg@kroah.com>,_Bill_Ryder_<bryder@sgi.com>,_Kuba_Ober_<kuba@mareimbrium.org>" \#define DRIVER_DESC "USB_FTDI_Serial_Converters_Driver"

static int debug;

static struct usb_device_id id_table_sio [] $=\{$

\{ USB_DEVICE (FTDI_VID, FTDI_SIO_PID) \}

USB_DEVICE(MOBILITY_VID, MOBILITY_USB_SERIAL_PID) $\}$

\} \{\}

/* Terminating entry */

* The 8U232AM has the same API as the sio except for

* - it can support MUCH higher baudrates; up to:

* o 921600 for RS232 and 2000000 for RS422/485 at $48 \mathrm{MHz}$

* o 230400 at $12 \mathrm{MHz}$

* so .. 8U232AM's baudrate setting codes are different

* - it has a two byte status code.

* - it returns characters every $16 \mathrm{~ms}$ (the FTDI does it every 40ms)

* the bcdDevice value is used to differentiate FT232BM and FT245BM from

* the earlier FT8U232AM and FT8U232BM. For now, include all known VID/PID

* combinations in both tables.

* FIXME: perhaps bcdDevice can also identify $12 \mathrm{MHz}$ devices, but I don't know

* if those ever went into mass production. [Ian Abbott]

$*$ /

static struct usb_device_id id_table_8U232AM [] $=\{$

\{ USB_DEVICE_VER(FTDI_VID, FTDI_IRTRANS_PID, $0,0 \times 3 \mathrm{ff})\}$,

USB_DEVICE_VER(FTDI_VID, FTDI_8U232AM_PID, $0,0 \times 3 \mathrm{ff})\}$,

USB_DEVICE_VER (FTDI_VID, FTDI_8U232AM_ALT_PID, $0,0 \times 3 \mathrm{ff}$ ) \},

USB_DEVICE_VER(FTDI_VID, FTDI_RELAIS_PID , 0 , $0 \times 3 \mathrm{ff})\}$

USB_DEVICE (INTERBIOMETRICS_VID, INTERBIOMETRICS_IOBOARD_PID) \},

USB_DEVICE(INTERBIOMETRICS_VID, INTERBIOMETRICS_MINI_IOBOARD_PID) \},

USB_DEVICE_VER(FTDI_NF_RIC_VID, FTDI_NF_RIC_PID, $0,0 \times 3 \mathrm{ff})\}$,

USB_DEVICE_VER(FTDI_VID, FTDI_XF_632_PID, $0,0 \times 3 \mathrm{ff}$ )

USB_DEVICE_VER(FTDI_VID, FTDI_XF_634_PID, $0,0 \times 3 \mathrm{ff}$ ) $\}$,

USB_DEVICE_VER(FTDI_VID, FTDI_XF_547_PID , $0,0 \times 3 \mathrm{ff})\}$

USB_DEVICE_VER(FTDI_VID, FTDI_XF_633_PID , $0,0 \times 3 \mathrm{ff})\}$

USB_DEVICE_VER(FTDIVID, FTDI_XF_631_PID, $0,0 \times 3 \mathrm{ff}$ )

USB_DEVICE_VER(FTDI_VID, FTDI_XF_635_PID, $0,0 \times 3 \mathrm{ff})\}$,

USB_DEVICE_VER(FTDI_VID, FTDI_XF_640_PID, $0,0 \times 3 \mathrm{ff})\}$

USB_DEVICE_VER(FTDI_VID, FTDI_XF_642_PID, $0,0 \times 3 \mathrm{ff}$ ) $\}$,

USB_DEVICE_VER(FTDI_VID, FTDI_VNHCPCUSB_D_PID, $0,0 \times 3 \mathrm{ff})\}$,

USB_DEVICE_VER(FTDI_VID, FTDI_DSS20_PID, $0,0 \times 3 \mathrm{ff}$ )

USB_DEVICE_VER(SEALEVEL_VID, SEALEVEL_2101_PID, $0,0 \times 3 \mathrm{ff})\}$,

USB_DEVICE_VER (SEALEVEL_VID, SEALEVEL_2102_PID, $0,0 \times 3 \mathrm{ff}$ ) \},

USB_DEVICE_VER(SEALEVEL_VID, SEALEVEL_2103_PID, $0,0 \times 3 \mathrm{ff}$ ) \},

USB_DEVICE_VER(SEALEVEL_VID, SEALEVEL_2104_PID, $0,0 \times 3 \mathrm{ff}$ ) \},

\{ USB_DEVICE_VER(SEALEVEL_VID, SEALEVEL_2201_1_PID, $0,0 \times 3 \mathrm{ff}$ ) \},

USB_DEVICE_VER(SEALEVEL_VID, SEALEVEL_2201_2_PID, $0,0 \times 3 \mathrm{ff})\}$,

USB_DEVICE_VER(SEALEVEL_VID，SEALEVEL_2202_1_PID， $0,0 \times 3 \mathrm{ff}$ ) \},

USB_DEVICE_VER(SEALEVEL_VID, SEALEVEL_2202_2_PID, $0,0 \times 3 \mathrm{ff}$ )

USB_DEVICE_VER(SEALEVEL_VID，SEALEVEL_2203_1_PID, $0,0 \times 3 \mathrm{ff})\}$,

USB_DEVICE_VER(SEALEVEL_VID，SEALEVEL_2203_2_PID, $0,0 \times 3 \mathrm{ff}$ ) \},

USB_DEVICE_VER (SEALEVEL_VID, SEALEVEL_2401_1_PID, $0,0 \times 3 \mathrm{ff}$ ) \},

USB DEVICE VER (SEALEVEL VID, SEALEVEL 2401_2_PID, $0,0 \times 3 \mathrm{ff}$ ) \},

USB_DEVICE_VER (SEALEVEL_VID, SEALEVEL_2401_3_PID, $0,0 \times 3 \mathrm{ff}$ )

USB_DEVICE_VER(SEALEVEL_VID, SEALEVEL_2401_4_PID, $0,0 \times 3 \mathrm{ff}$ ) \},

USB_DEVICE_VER(SEALEVEL_VID, SEALEVEL_2402_1_PID， $0,0 \times 3 \mathrm{ff}$ )

USB_DEVICE_VER(SEALEVEL_VID，SEALEVEL_2402_2_PID, $0,0 \times 3 \mathrm{ff}$ )

USB_DEVICE_VER(SEALEVEL_VID, SEALEVEL_2402_3_PID, $0,0 \times 3 \mathrm{ff}$ ) $\}$,

USB_DEVICE_VER(SEALEVEL_VID，SEALEVEL_2402_4_PID, $0,0 \times 3 \mathrm{ff}$ ) \},

USB_DEVICE_VER(SEALEVEL_VID, SEALEVEL_2403_1_PID, $0,0 \times 3 \mathrm{ff}$ ) \},

USB DEVICE VER (SEALEVEL VID, SEALEVEL 2403_2 PID, $0,0 \times 3 \mathrm{ff}$ )

USB DEVICE VER(SEALEVEL VID SEALEVEL 2403 3 PID, $0,0 \times 3 \mathrm{ff}$ )

USB_DEVICE_VER(SEALEVEL_VID, SEALEVEL_2403_4_PID， $0,0 \times 3 \mathrm{ff}$ ) \},

USB_DEVICE_VER(SEALEVEL_VID, SEALEVEL_2801_1_PID, $0,0 \times 3 \mathrm{ff})\}$,

USB_DEVICE_VER(SEALEVEL_VID，SEALEVEL_2801_2_PID, $0,0 \times 3 \mathrm{ff}$ ) \},

USB_DEVICE_VER(SEALEVEL_VID, SEALEVEL_2801_3_PID, 0, $0 \times 3 \mathrm{ff}$ ) \},

USB_DEVICE_VER (SEALEVEL_VID, SEALEVEL_2801_4_PID, $0,0 \times 3 \mathrm{ff}$ ) \},

USB_DEVICE_VER(SEALEVEL_VID，SEALEVEL_2801_5_PID， $0,0 \times 3 \mathrm{ff}$ )

USB_DEVICE_VER(SEALEVEL_VID，SEALEVEL_2801_6_PID，0，0x3ff) \},

USB DEVICE_VER (SEALEVEL_VID, SEALEVEL_2801 7 PID, $0,0 \times 3 \mathrm{ff})\}$,

USB_DEVICE_VER(SEALEVEL_VID, SEALEVEL_2801_8_PID, $0,0 \times 3 \mathrm{ff})\}$,

USB_DEVICE_VER(SEALEVEL_VID, SEALEVEL_2802_1_PID, $0,0 \times 3 \mathrm{ff})\}$,

USB_DEVICE_VER(SEALEVEL_VID, SEALEVEL_2802_2_PID, $0,0 \times 3 \mathrm{ff}$ ) \},

USB_DEVICE_VER (SEALEVEL_VID, SEALEVEL_2802_3_PID, $0,0 \times 3 \mathrm{ff}$ )

USB_DEVICE_VER(SEALEVEL_VID, SEALEVEL_2802_4_PID, $0,0 \times 3 \mathrm{ff}$ ) \},

USB_DEVICE_VER(SEALEVEL_VID, SEALEVEL_2802_5_PID, $0,0 \times 3 \mathrm{ff}$ ) \},

USB_DEVICE_VER(SEALEVEL_VID，SEALEVEL_2802_6_PID， $0,0 \times 3 \mathrm{ff}$ ) \},

USB_DEVICE_VER(SEALEVEL_VID, SEALEVEL_2802_7_PID, $0,0 \times 3 \mathrm{ff}$ ) ,

\{ USB_DEVICE_VER(SEALEVEL_VID, SEALEVEL_2802_8_PID, $0,0 \times 3 \mathrm{ff}$ ) \}, 


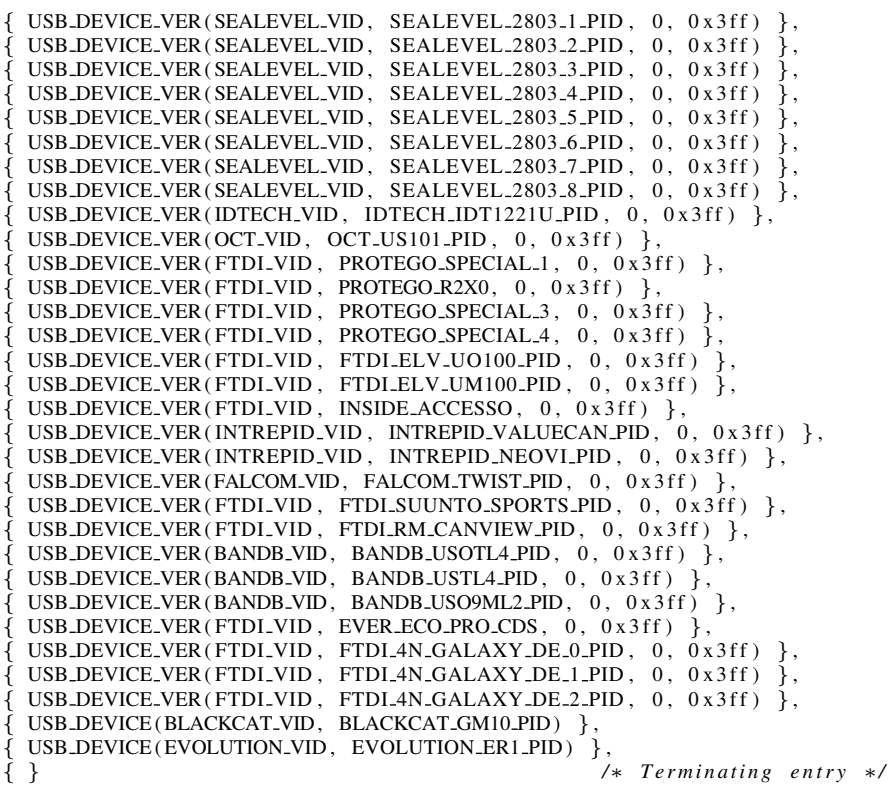
\};

static struct usb_device_id id_table_FT232BM [] $=\{$

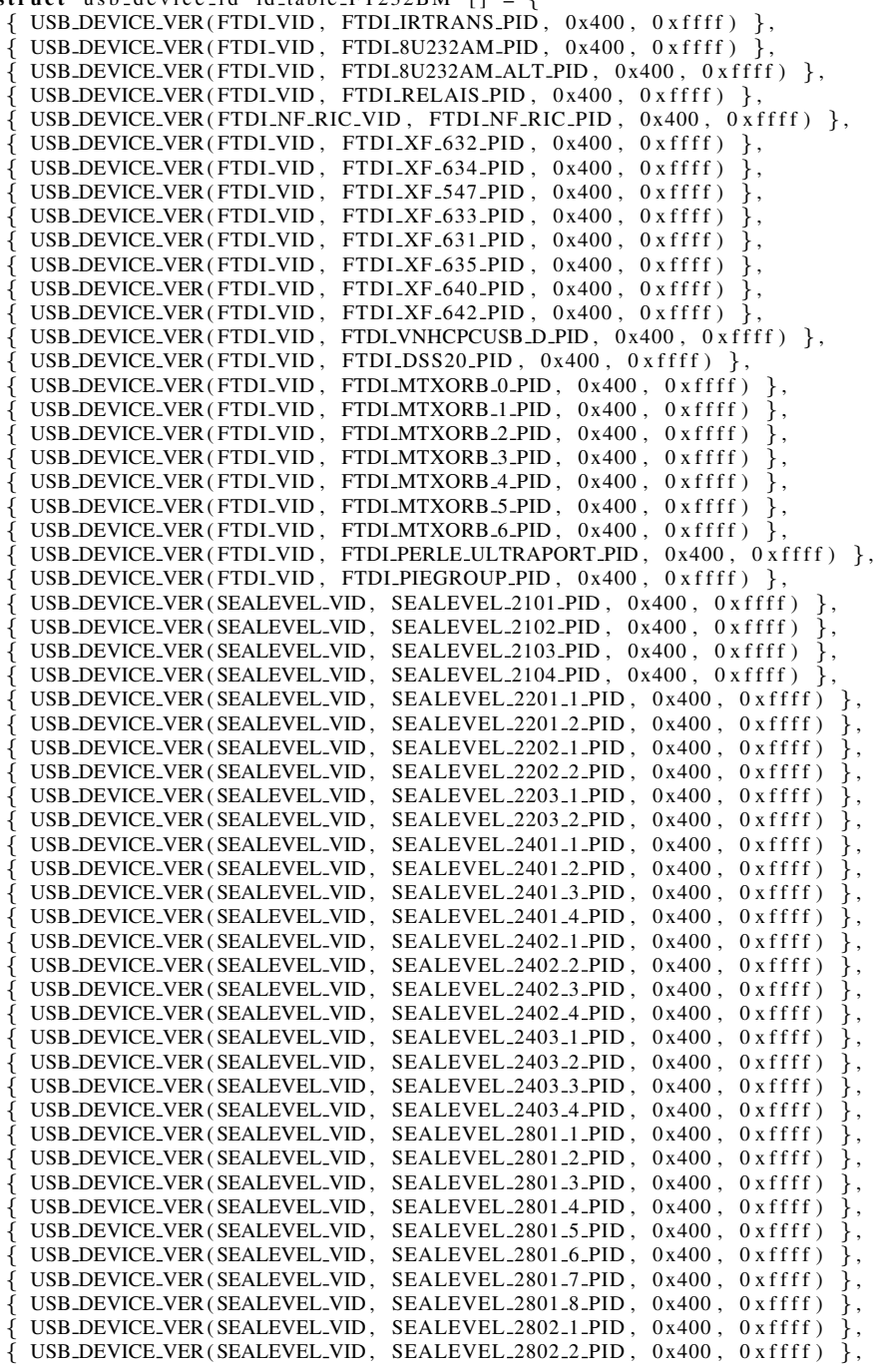


\{ USB_DEVICE_VER(SEALEVEL_VID, SEALEVEL_2802_3_PID, $0 \times 400,0 \times \mathrm{ffff}$ ) $\}$, \{ USB_DEVICE_VER(SEALEVEL_VID, SEALEVEL_2802_4_PID, $0 \times 400,0 \times \mathrm{ffff}$ ) \}, USB_DEVICE_VER (SEALEVEL_VID, SEALEVEL_2802_5_PID, $0 \times 400,0 \mathrm{xffff}$ ) \}, USB_DEVICE_VER(SEALEVEL_VID，SEALEVEL_2802_6_PID, 0x400, $0 \times \mathrm{xffff}$ ) , USB_DEVICE_VER (SEALEVEL_VID, SEALEVEL_2802_7_PID , 0x400, $0 \mathrm{xffff}$ ) \}, USB_DEVICE_VER(SEALEVEL_VID, SEALEVEL_2802_8 PID, $0 \times 400,0 \times \mathrm{ffff}$ ) , USB DEVICE_VER(SEALEVEL_VID, SEALEVEL 2803_1_PID, $0 \times 400,0 \times \mathrm{ffff})\}$, USB_DEVICE_VER(SEALEVEL_VID, SEALEVEL_2803___PID, $0 \times 400,0 \mathrm{xffff}$ ) USB_DEVICE_VER(SEALEVEL_VID，SEALEVEL_2803_3_PID, 0x400, 0 xfffff) \}, USB_DEVICE_VER(SEALEVEL_VID，SEALEVEL_2803_4_PID，0x400，0xffff) \}, USB_DEVICE_VER(SEALEVEL_VID, SEALEVEL_2803_5_PID, 0x400, $0 \mathrm{xffff}$ ) \}, USB_DEVICE_VER(SEALEVEL_VID, SEALEVEL_2803_6_PID, 0x400, $0 \mathrm{xfffff}$ ) USB_DEVICE_VER(SEALEVEL_VID, SEALEVEL_2803_7_PID, 0x400, $0 \mathrm{xffff}$ ) USB_DEVICE_VER(SEALEVEL_VID, SEALEVEL_2803_8_PID, 0x400, $0 \mathrm{xffff}$ ) \}, USB_DEVICE_VER (IDTECH VID, IDTECH_IDT1221U_PID, $0 \times 400,0 \times \mathrm{ffff}$ ) USB_DEVICE_VER(OCT_VID, OCT_US101_PID, $0 \times 400,0 \mathrm{xffff})\}$, USB_DEVICE_VER(FTDI_VID, PROTEGO_SPECIAL_1, 0x400, 0 xffff ) $\}$, USB_DEVICE_VER (FTDI_VID, PROTEGO_R2X0，0x400，0 xffff ) \}, USB_DEVICE_VER (FTDI_VID, PROTEGO_SPECIAL_3, 0x400, $0 \mathrm{xffff}$ ) USB_DEVICE_VER(FTDI_VID，PROTEGO_SPECIAL_4, $0 \times 400,0 \mathrm{xffff}$ ) USB_DEVICE_VER(FTDI_VID, FTDI_GUDEADS_E808_PID, 0x400, 0 xffff ) \}, USB_DEVICE_VER(FTDI_VID, FTDI_GUDEADS_E809_PID, $0 \times 400,0 \mathrm{xffff}$ ) USB DEVICE VER(FTDIVID, FTDI_GUDEADS_E80A_PID, $0 \times 400,0 \times \mathrm{ffff})\}$ USB_DEVICE_VER(FTDI_VID, FTDI_GUDEADS_E80B_PID, $0 \times 400,0 \times \mathrm{ffff}$ ) $\}$, $\left.\begin{array}{llll}\text { USB_DEVICE_VER(FTDI_VID, FTDI_GUDEADS_E80B_PID, } & 0 \times 400, & 0 \times \mathrm{ffff} \text { ) } \\ \text { USB_DEVICE_VER(FTDI_VID, FTDI_GUDEADS_E80C_PID, } & 0 \times 400,0 \times \mathrm{ffff})\end{array}\right\}$, USB_DEVICE_VER(FTDI_VID, FTDI_GUDEADS_E80D_PID, 0x400, $0 \mathrm{xffff}$ ) USB_DEVICE_VER(FTDI_VID，FTDI_GUDEADS_E80E_PID，0x400，0 xffff) \}, USB_DEVICE_VER(FTDI_VID, FTDI_GUDEADS_E80F_PID，0x400，0xffff) USB_DEVICE_VER(FTDI_VID, FTDI_GUDEADS_E888_PID，0x400，0xffff) USB_DEVICE_VER(FTDI_VID, FTDI_GUDEADS_E889_PID, $0 \times 400,0 \mathrm{xffff}$ ) USB_DEVICE_VER(FTDI_VID, FTDI_GUDEADS_E88A_PID, $0 \times 400,0 \mathrm{xffff}$ ) USB_DEVICE_VER (FTDI_VID, FTDI_GUDEADS_E88B_PID, $0 \times 400,0 \mathrm{xffff}$ ) USB_DEVICE_VER (FTDI_VID,FTDI_GUDEADS_E88C_PID, $0 \times 400,0 \mathrm{xffff}$ ) USB_DEVICE_VER(FTDI_VID, FTDI_GUDEADS_E88D_PID, $0 \times 400,0 \mathrm{xffff})\}$ USB_DEVICE_VER(FTDI_VID, FTDI_GUDEADS_E88E_PID, $0 \times 400,0 \mathrm{xffff})\}$, USB_DEVICE_VER(FTDI_VID, FTDI_GUDEADS_E88F_PID, $0 \times 400,0 \mathrm{xffff}$ ) USB_DEVICE_VER(FTDI_VID, FTDI_ELV_UO100_PID, $0 \times 400,0 \mathrm{xffff}$ ) \}, USB_DEVICE_VER(FTDI_VID, FTDI_ELV_UM100_PID, $0 \times 400,0 \mathrm{xffff}$ ) USB_DEVICE_VER(FTDI_VID, LINX_SDMUSBQSS_PID, $0 \times 400,0 \mathrm{xffff}$ ) $\}$, \{ USB_DEVICE_VER(FTDI_VID, LINX_MASTERDEVEL2_PID, $0 \times 400,0 \mathrm{xffff}$ ) \{ USB_DEVICE_VER(FTDI_VID，LINX_FUTURE_0_PID，0x400，0 xffff) \}, USB_DEVICE_VER(FTDI_VID, LINX_FUTURE_1_PID, $0 \times 400,0 \mathrm{xffff})\}$ \{ USB_DEVICE_VER(FTDI_VID, LINX_FUTURE_2_PID, 0x400, $0 \mathrm{xffff}$ ) USB_DEVICE(FTDI_VID, FTDI_CCSICDU20_0_PID) ,

static struct usb_device_id id_table_USB_UIRT [ ] $=\{$ \{ USB_DEVICE(FTDI_VID, FTDI_USB_UIRT_PID) $\}$

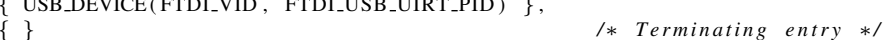
\}

static struct usb_device_id id_table_HE_TIRA1 [] = \{ USB_DEVICE_VER(FTDI_VID, FTDI_HE_TIRA1_PID, $0 \times 400,0 \mathrm{xffff}$ ) \}; \{\}

/* Terminating entry */

static struct usb_device_id id_table_FT2232C[] $=\{$ \{ USB_DEVICE(FTDI_VID, FTDI_8U2232C_PID) \}, \{\}

\}

static struct usb_device_id id_table_combined [] $=\{$

\{ USB DEVICE(FTDI VID, FTDI IRTRANS PID ) \},

USB_DEVICE(FTDI_VID, FTDI_SIO_PID ) \}

USB_DEVICE(FTDI_VID, FTDI_8U232AM_PID) \},

\{ USB_DEVICE(FTDI_VID, FTDI_8U232AM_ALT_PID) \},

USB_DEVICE (FTDI_VID, FTDI_8U2232C_PID ) \},

USB_DEVICE(FTDI_VID, FTDI_RELAIS_PID )

USB_DEVICE(INTERBIOMETRICS_VID, INTERBIOMETRICS_IOBOARD_PID ) \},

\{ USB_DEVICE (INTERBIOMETRICS_VID，INTERBIOMETRICS_MINI_IOBOARD_PID) \}

\{ USB_DEVICE(FTDI_VID , FTDI_XF_632_PID) \},

\{ USB_DEVICE(FTDI_VID, FTDI_XF_634_PID) \}, 


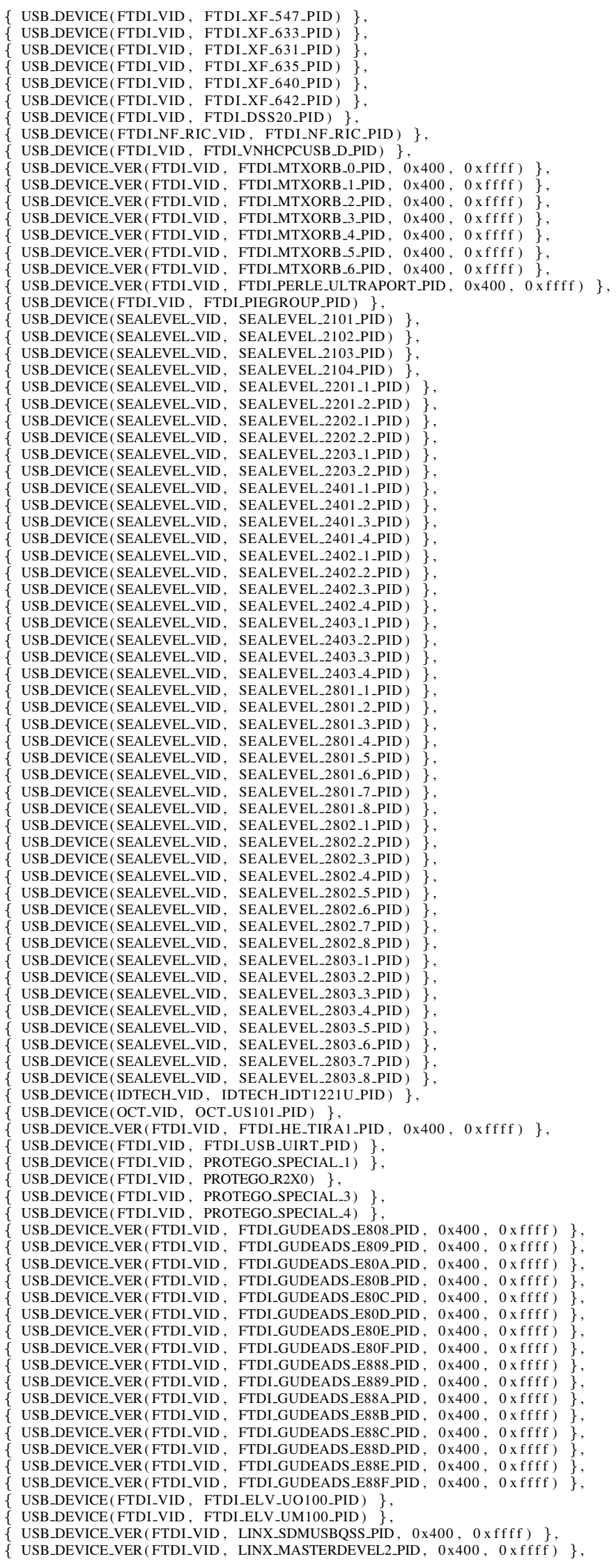




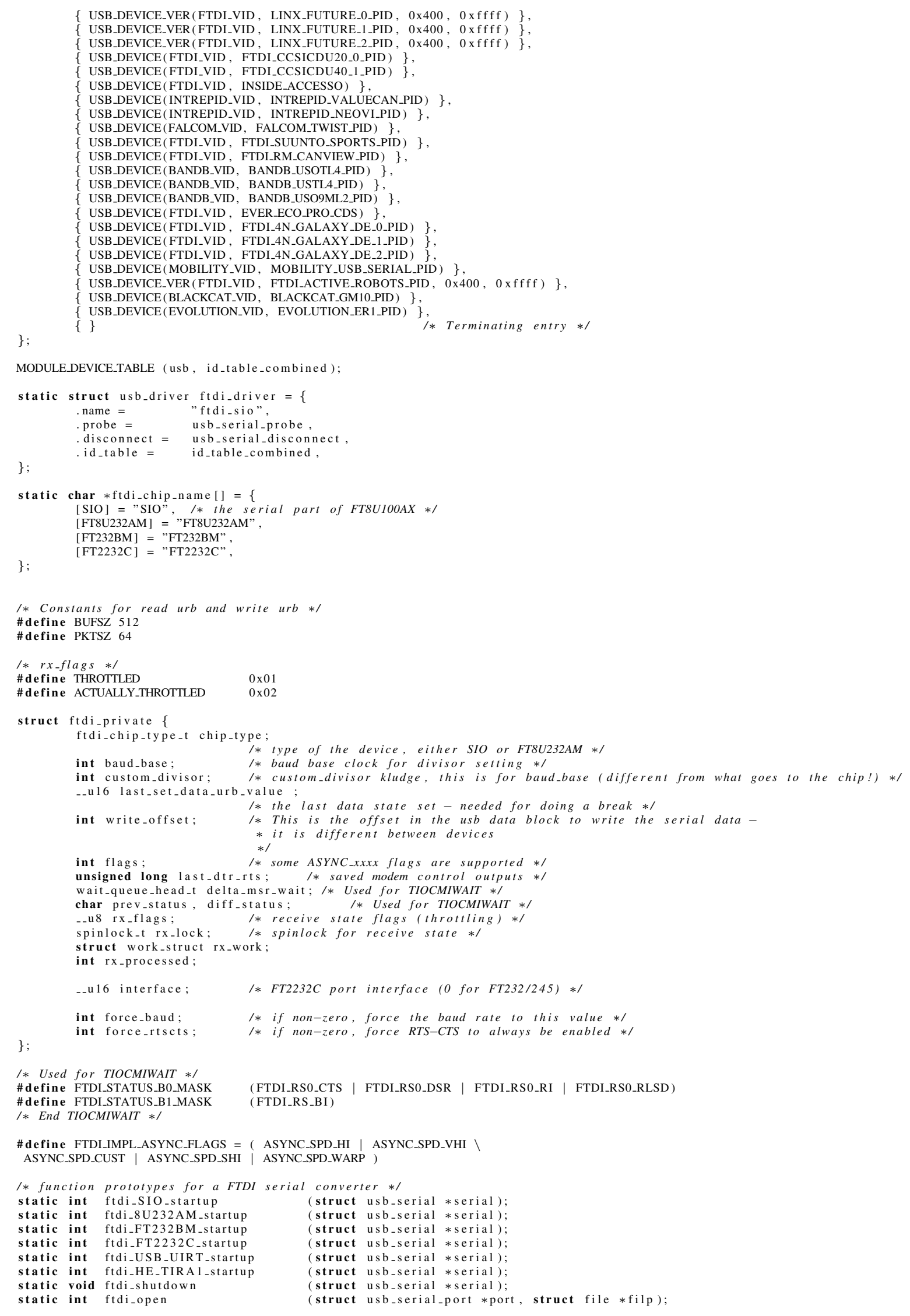


static void ftdi_close static int ftdi-write static int ftdi-write_room static int ftdi_chars_in_buffer

static void ftdi_write_bulk_callback static void ftdi_read_bulk_callback

static void ftdi_process_read

static void ftdi-set termios

static int ftdi_tiocmget

static int ftdi_tiocmset

static int ftdi_ioctl

static void ftdi_break_ctl

static void ftdi_throttle

static void ftdi_unthrottle (struct usb_serial_port *port, struct file *filp);

(struct usb_serial_port *port, const unsigned char $*$ buf, int count);

(struct usb_serial_port * port);

(struct usb_serial_port * port);

(struct urb *urb, struct pt_regs *regs);

(struct urb *urb, struct pt_regs *regs);

(void * param);

(struct usb_serial_port $*$ port, struct termios $*$ old);

(struct usb_serial_port $*$ port, struct file $*$ file);

(struct usb_serial_port *port, struct file * file, unsigned int set, unsigned int clear);

(struct usb_serial_port *port, struct file * file, unsigned int cmd, unsigned long arg);

(struct usb_serial_port *port, int break_state) ;

(struct usb_serial_port * port);

(struct usb_serial_port * port);

static unsigned short int ftdi_232am_baud_base_to_divisor (int baud, int base);

static unsigned short int ftdi_232am_baud_to_divisor (int baud);

static _- 32 ftdi_232bm_baud_base_to_divisor (int baud, int base)

static _-u 32 ftdi_232bm_baud_to_divisor (int baud);

static struct usb serial device type ftdi_SIO device $=\{$

owner $=\quad$ THIS_MODULE

name $=\quad$ "FTDI_SIO",

id_table_sio

num_interrupt_in $=0$

num_bulk_in $=\quad 1$

num_bulk_out $=\quad 1$,

$\begin{array}{ll}\text { open }= & \text { ftdi_open }\end{array}$

close $=$ ftdi close

throttle = ftdi-throttle

unthrottle $=\quad$ ftdi-unthrottle

write $=\quad$ ftdi-write

write_room $=\quad$ ftdi_write_room,

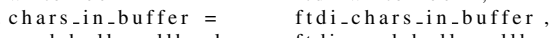

read_bulk_callback $=$ ftdi_read_bulk_callback,

write_bulk_callback = ftdi_write_bulk_callback

tiocmget $=\quad$ ftdi_tiocmget,

tiocmset $=$ ftdi_tiocmset,

ioctl $=\quad$ ftdi_ioctl

set_termios $=$ ftdi_set_termios,

break_ctl $\quad$ ftdi_break_ctl,

attach = ftdi_SIO_startup

\}

shutdown $=$ ftdi_shutdown,

static struct usb_serial_device_type ftdi_8U232AM_device $=\{$

.owner $=$

name $=$

id_table $=$

HIS_MODULE,

inum_interrupt_in $=0$

num_bulk_in $=\quad 1$,

num_bulk_out $=$

open $=$ ftdi_open

close $=$ ftdi_close

throttle $=\quad$ ftdi_throttle

unthrottle $=$ ftdi-unthrottle

write $=\quad$ ftdi_write

write_room $=$ ftdi_write_room,

chars_in_buffer $=\quad$ ftdi_chars_in_buffer,

read_bulk_callback = ftdi_read_bulk_callback,

write_bulk_callback $=$ ftdi_write_bulk_callback

tiocmget $=\quad$ ftdi_tiocmget,

tiocmset $=\quad$ ftdi_tiocmset

ioctl $=\quad$ ftdi_ioctl

set_termios = ftdi_set_termios,

break_ctl = ftdi_break_ctl

attach = ftdi_8U232AM_startup,

\} ;

ftdi_shutdown,

static struct usb_serial_device_type ftdi_FT232BM_device $=\{$

owner $=$
name $=$

id_table $=$

THIS_MODULE,

num_bulk_in $=$

num_bulk_out $=\quad 1$,

num_ports =

open $=$

close $=$

throttle $=$

unthrottle

write $=$

write_room =

chars_in_buffer =

read_bulk_callback =

write_bulk_callback =

tiocmget $=$

tiocmset $=$

ioctl $=$

"FTDI_FT232BM_Compatible",

ftdi_open,

ftdi_close,

ftdi-throttle,

ftdi_unthrottle,

ftdi_write,

ftdi_write_room ,

ftdi_chars_in_buffer,

tdi_read_bulk_callback,

ftdi_write_bulk_callback

ftdi_tiocmget,

ftdi_tiocmset,

ftdi_ioctl 


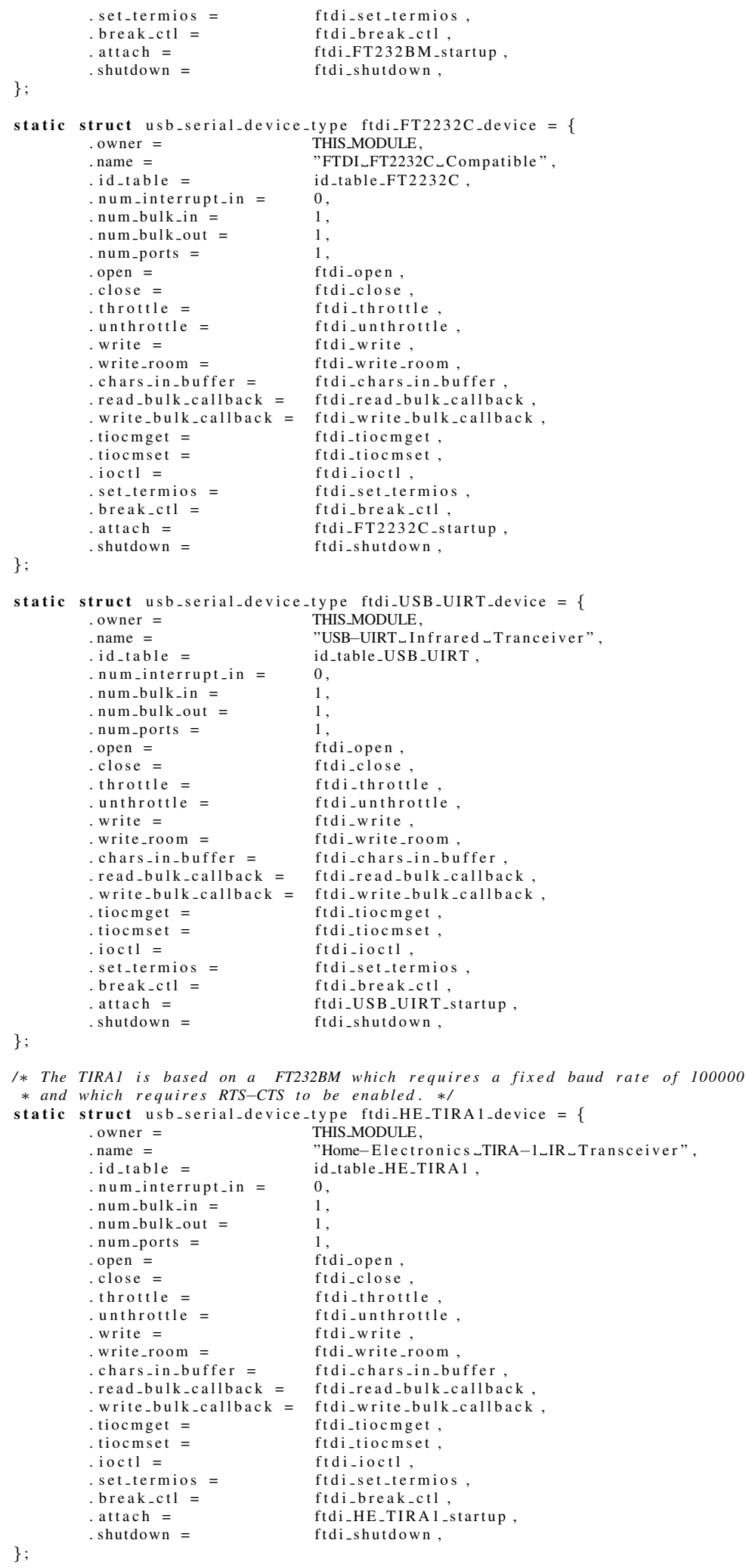

1* The TIRAl is based on a FT232BM which requires a fixed baud rate of 100000 * and which requires RTS-CTS to be enabled. *I

\#define WDR_TIMEOUT $5000 / *$ default urb timeout $* /$

/* High and low are for DTR, RTS etc etc */ \#define $\mathrm{HIGH} 1$ 


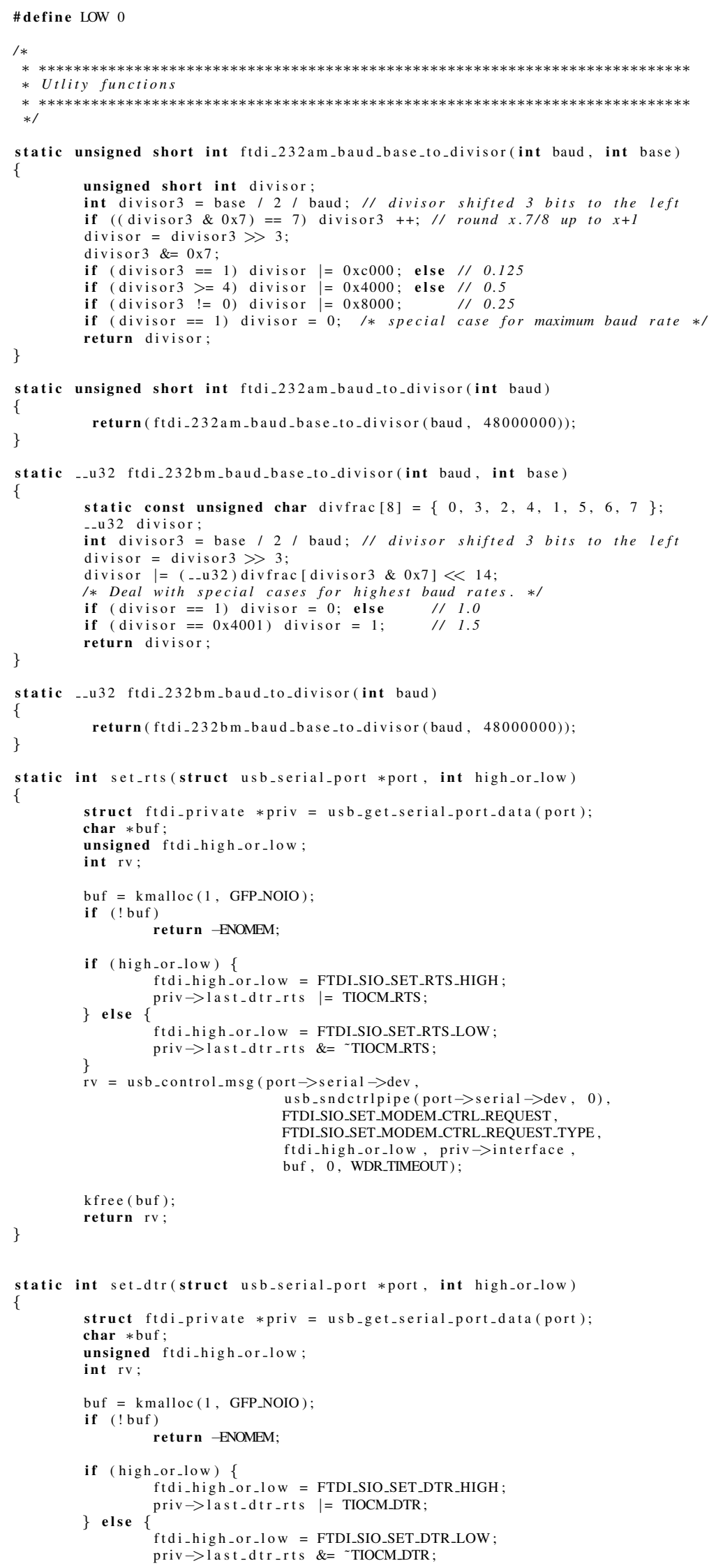


\}

$\mathrm{rv}=\mathrm{usb} \_$control_msg $($port $\rightarrow$ serial $\rightarrow$ dev

usb_sndctrlpipe (port $\rightarrow$ serial $\rightarrow$ dev, 0$)$

FTDI_SIO_SET_MODEM_CTRL_REQUEST

FTDI_SIO_SET_MODEM_CTRL_REQUEST_TYPE,

ftdi_high_or_low, priv $\rightarrow$ interface ,

buf , 0 , WDR_TIMEOUT):

kfree (buf)

return rv;

\}

static _-u32 get_ftdi_divisor(struct usb_serial_port $*$ port);

static int change_speed(struct usb_serial_port *port)

\{

struct ftdi_private $*$ priv $=u_{s b}$ get_serial_port_data(port);

char *buf;

_-u 16 urb_value;

_- u 16 urb_index;

u32 urb index value :

int $\mathrm{rv}$;

buf $=\operatorname{kmalloc}(1$, GFP_NOIO $)$

if (! buf)

return $-\mathrm{ENOMEM}$

urb_index_value $=$ get_ftdi_divisor $($ port $)$

urb_value $=\left({ }_{-}\right.$u16) urb_index_value;
urb_index $=\left(\right.$- $\left._{1} 16\right)\left(\right.$ urb_index_value $\left._{-}>16\right) ;$

urb_index $=($ _-u 16$)\left(\right.$ urb_index_value $_{-}>$
if $($priv $\rightarrow$ interface) $\{/ *$ FT2232C */

\}

urb_index $=\left(\right.$ _-u16) $_{1}(($ urb_index $<<8) \mid$ priv $\rightarrow$ interface $)$;

$r v=u s b \_c o n t r o l \_m s g($ port $\rightarrow$ serial $\rightarrow$ dev,

usb_sndctrlpipe (port $\rightarrow$ serial $\rightarrow$ dev, 0$)$

FTDI_SIO_SET_BAUDRATE_REQUEST,

FTDI_SIO_SET_BAUDRATE_REQUEST_TYPE,

urb_value, urb_index

buf, 0,100$)$;

kfree (buf)

return rv;

static _-_ 32 get_ftdi_divisor (struct usb_serial_port * port)

$\{$ I get_ftdi_divisor $*\}$

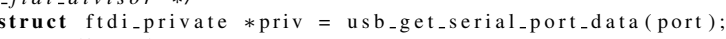

--u32 div_value $=0$

int div_okay = 1 ;

int baud

* The logic involved in setting the baudrate can be cleanly split in 3 steps.

* Obtaining the actual baud rate is a little tricky since unix traditionally

* somehow ignored the possibility to set non-standard baud rates.

* 1. Standard baud rates are set in tty $\rightarrow$ termios $\rightarrow c_{-}$cflag

* 2. If these are not enough, you can set any speed using alt_speed as follows:

* - set tty $\rightarrow$ termios $\rightarrow c_{-}$cflag speed to B38400

* - set your real speed in tty->alt_speed; it gets ignored when

alt_speed $==0$, $($ or $)$

- call TIOCSSERIAL ioctl with (struct serial_struct) set as follows:

flags \& ASYNC_SPD_MASK == ASYNC_SPD_[HI, VHI, SHI, WARP], this just

* flags \& ASYNC_SPD_MASK == ASYNC_SPD_[HI, VHI, SHI, WARPJ, this just

* sets alt_speed to (HI: 57600, VHI: 115200, SHI:
$* * *$ Steps 1, 2 are done courtesy of tty_get_baud_rate

* 3. You can also set baud rate by setting custom divisor as follows

* - set tty $\rightarrow$ termios $\rightarrow c_{-}$cflag speed to B38400

* - call TIOCSSERIAL ioctl with (struct serial_struct) set as follows:

o flags \& ASYNC_SPD_MASK $==$ ASYNC_SPD_CUST

o custom_divisor set to baud_base / your_new_baudrate

* ** Step 3 is done courtesy of code borrowed from serial.c - I should really

* spend some time and separate+move this common code to serial.c, it is

* replicated in nearly every serial driver you see.

1* 1. Get the baud rate from the tty settings, this observes alt_speed hack */

baud = tty_get_baud_rate (port $\rightarrow$ tty $)$;

dbg ("\%s_-_tty_get_baud_rate_reports_speed $\%$ d", ,_FUNCTION_-, baud );

I* 2. Observe async-compatible custom_divisor hack, update baudrate if needed */

// MGW: ADDED PER EVOLUTION_RCM MODULE

if $($ baud $==230400) / * \& \&$ port $\rightarrow$ serial $\rightarrow$ product $==$ EVO_HYBRID_PID $) * /$

baud $=250000$

\}

dbg ("\%s : _bumped_magical_230400_baud_to _ $2.5 \mathrm{~kb}$, , _._FUNCTION__ )

//MGW: End of additions 
if $($ baud $==38400 \& \&$

$(($ priv $\rightarrow$ flags \& ASYNC_SPD_MASK $)==$ ASYNC_SPD_CUST $) \& \&$

$($ priv $\rightarrow$ custom_divisor ) \{

baud $=$ priv $\rightarrow$ baud_base $/$ priv $\rightarrow$ custom_divisor

\}

dbg ("\%s_-_custom_divisor_\%d_sets_baud_rate_to_\%d", _.FUNCTION__, priv $\rightarrow$ custom_divisor, baud);

I* 3. Convert baudrate to device-specific divisor */

if $($ ! baud ) baud $=9600$;

switch (priv $\rightarrow$ chip_type)

case SIO: /* SIO chip */

switch (baud) \{

case 300: div_value $=$ ftdi sio $b 300 ;$ break;

case 600: div_value $=$ ftdi_sio_b600; break

case 1200: div_value $=$ ftdi_sio_b $1200 ;$ break

case 2400: div_value $=f_{t d i}$ sio_b2 $_{-} 400$; break

case 4800: div_value $=\mathrm{ftdi}_{-} \mathrm{sio}_{-} \mathrm{b} 4800$; break

case 9600: div_value $=$ ftdi_sio_b9600; break

case 19200: div_value = ftdi_sio_b19200; break;

case 38400: div_value $=$ ftdi_sio_b38400; break;

case 57600: div_value $=$ ftdi_sio_b57600; break;

case 115200: div_value $=$ ftdi_sio_b $115200 ;$ break;

\} / * baud $* /$

if $($ div_value $=0)\{$

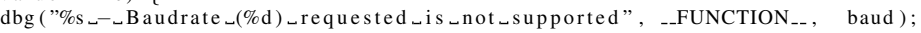

\}reak div_value $=$ ftdi_sio_b9600;

break ;

case FT8U232AM: /* 8U232AM chip */

if (baud $<=3000000$ )

\} else \{ div_value $=$ ftdi_232am_baud_to_divisor(baud);

dbg ("\%s_-_Baud $\lrcorner$ rate $\lrcorner$ too $\lrcorner$ high ! ”, _-FUNCTION_-- $)$ div_value $=$ ftdi_232am_baud_to_divisor (9600);

\} div_okay $=0$

break

case FT232BM: /* FT232BM chip */

case $\mathrm{F}$

if (baud $<=3000000$ )

\} else \{ div_value = ftdi_232bm_baud_to_divisor(baud);

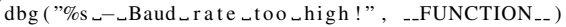
div_value $=$ ftdi_232bm_baud_to_divisor $(9600)$;

\} div_okay $=0$

\} /* priv $\rightarrow$ chip_type */

if (div_okay) \{

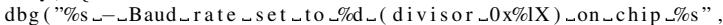

_._FUNCTION_-, baud, (unsigned long) div_value

\} ftdi_chip_name [priv $\rightarrow$ chip_type ])

\}

return(div_value);

static int get_serial_info(struct usb_serial_port $*$ port, struct serial_struct__user $*$ retinfo)

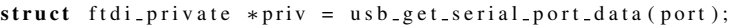
struct serial_struct tmp

if ( ! retinfo)

return -EFAULT;

$\operatorname{memset}(\& \mathrm{tmp}, \quad 0, \quad$ sizeof $(\mathrm{tmp}))$

tmp.flags $=$ priv $\rightarrow$ flags

tmp. baud_base $=$ priv $\rightarrow$ baud_base

tmp. custom_divisor $=$ priv $\rightarrow$ custom_divisor

if (copy_to_user(retinfo, \&tmp, sizeof $(*$ retinfo $))$

return 0

\} /* get_serial_info */

static int set_serial_info(struct usb_serial_port $*$ port, struct serial_struct _._user $*$ newinfo)

$\{1 *$ set_serial_info $*$ \}

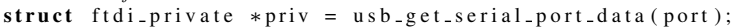

struct serial_struct new_serial;

struct ftdi-private old_priv;

if (copy_from_user(\&new_serial, newinfo, sizeof(new_serial)))

return -EFAULT;

old_priv $=*$ priv

/* Do error checking and permission checking */

if (! capable (CAP_SYS_ADMIN)) \{ 


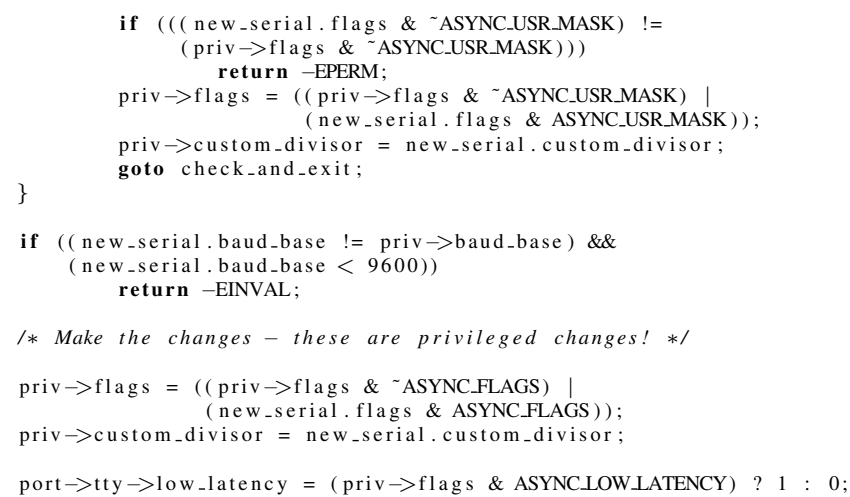




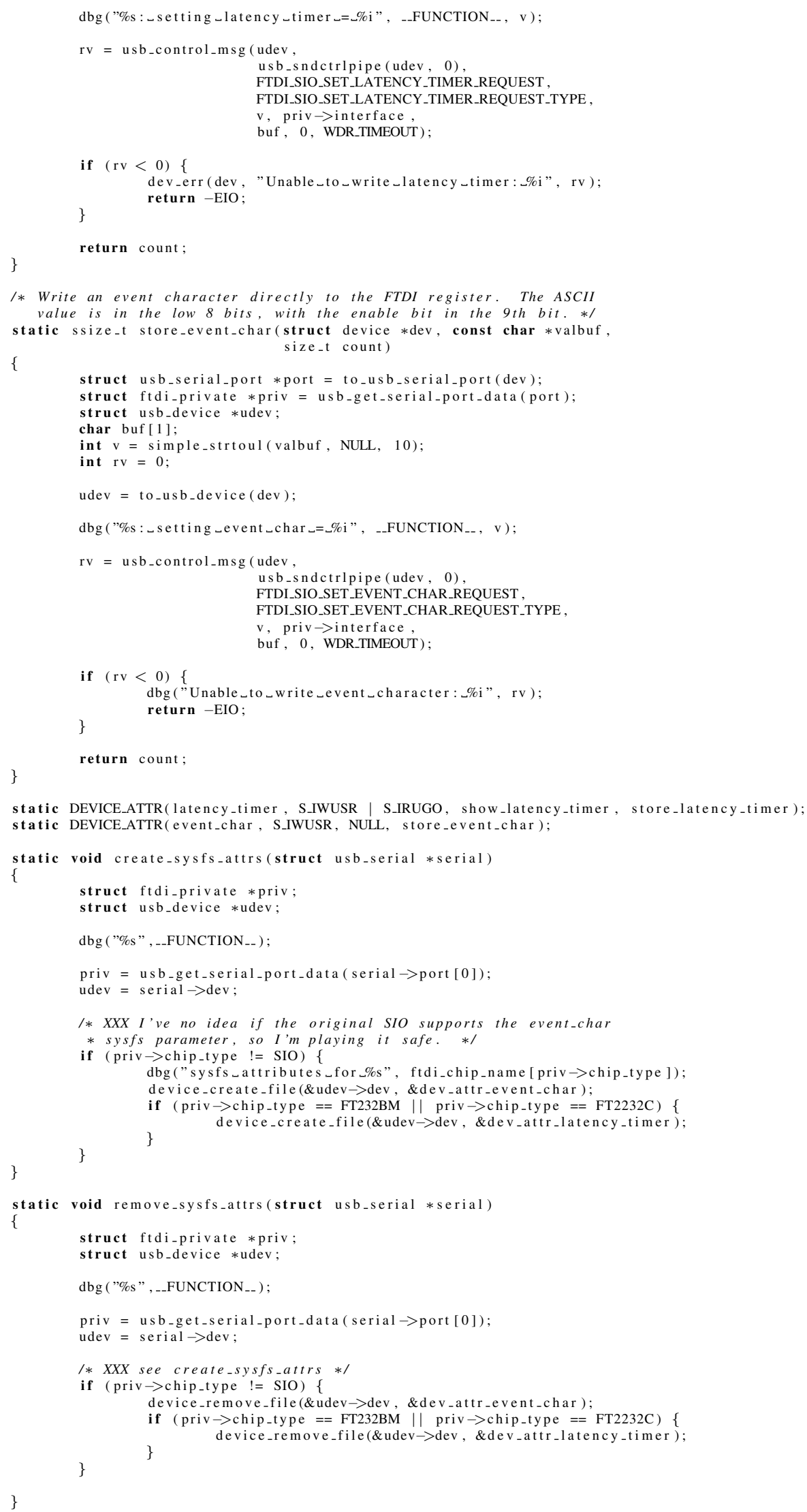




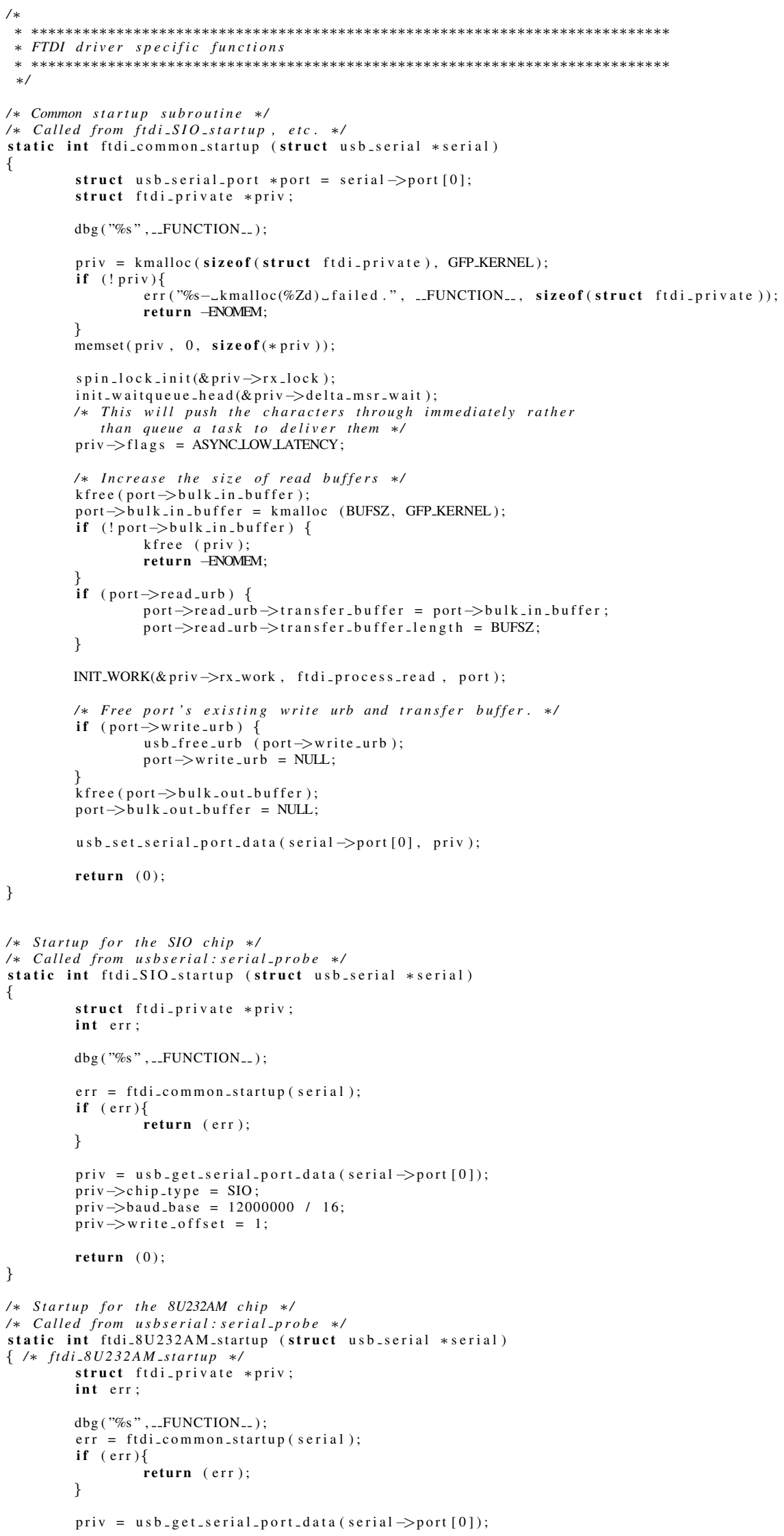




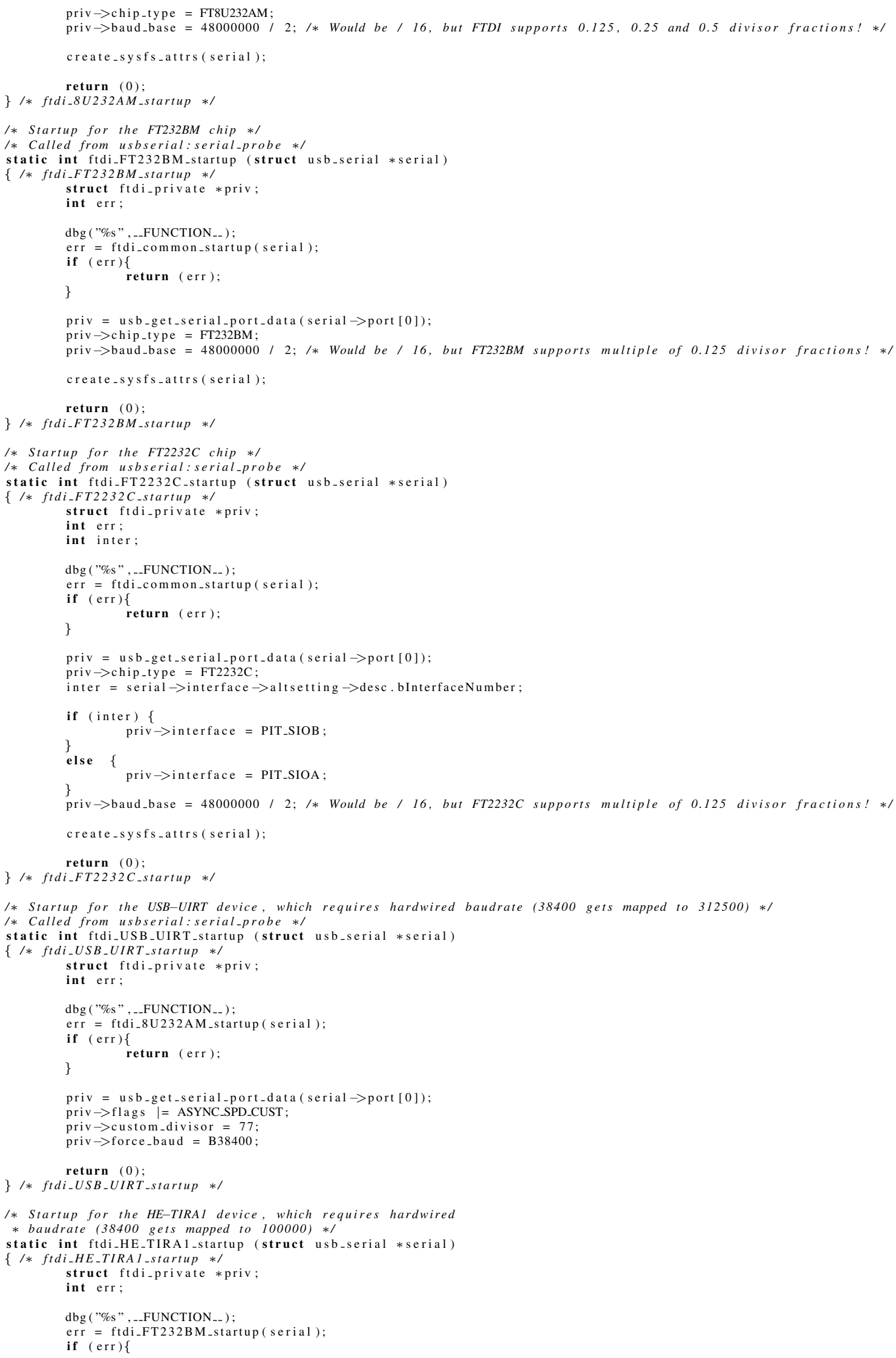




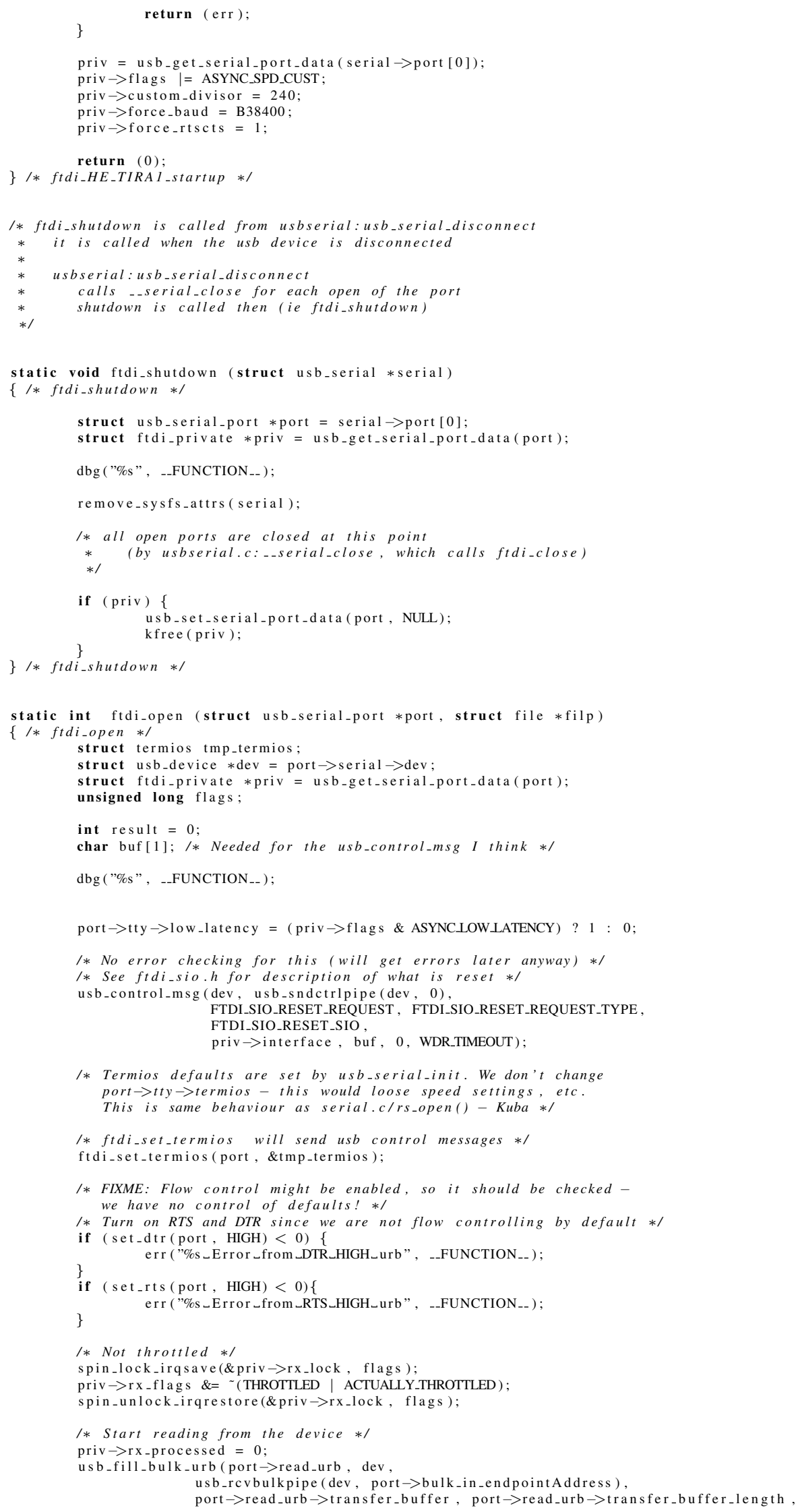




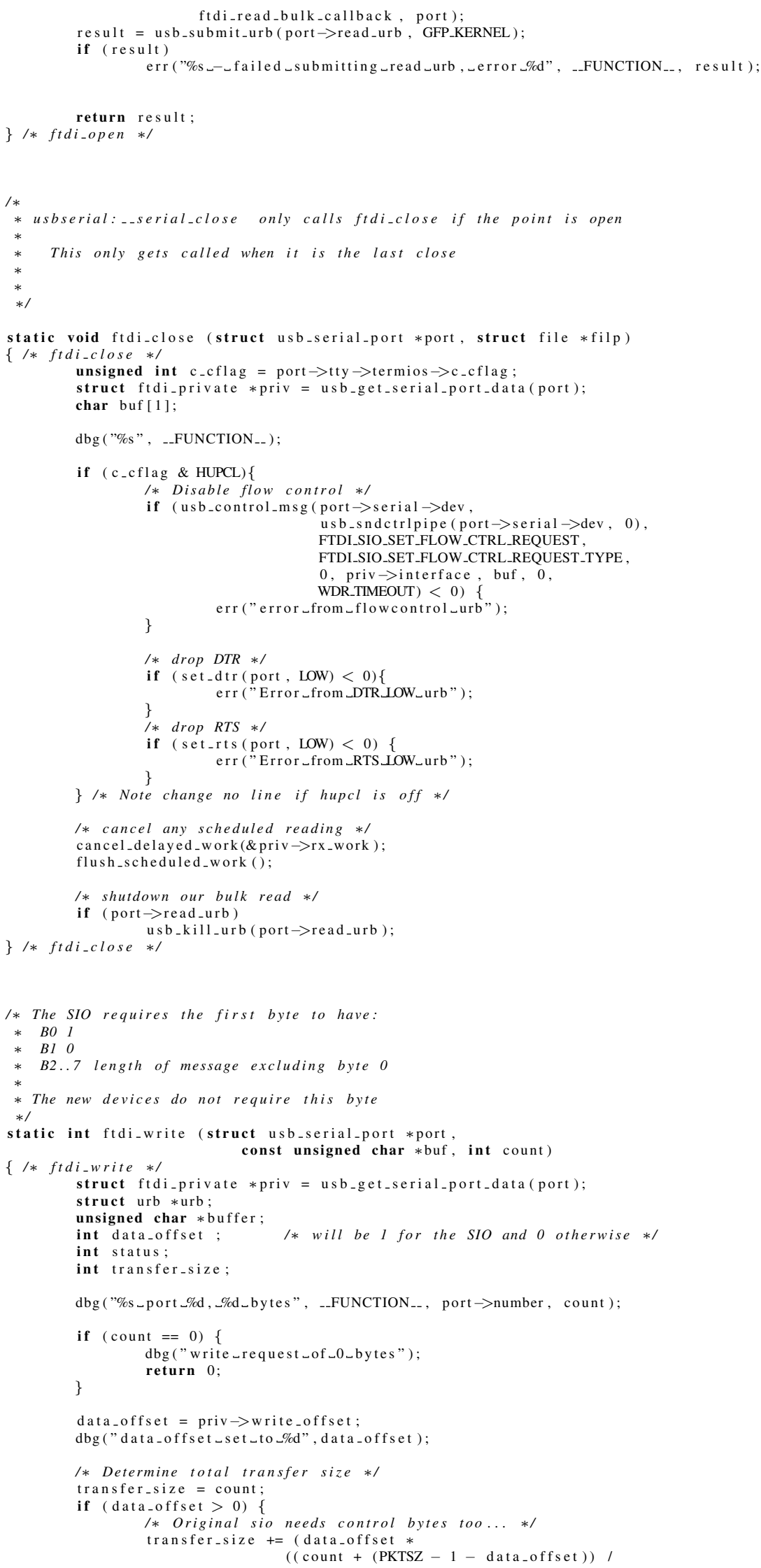




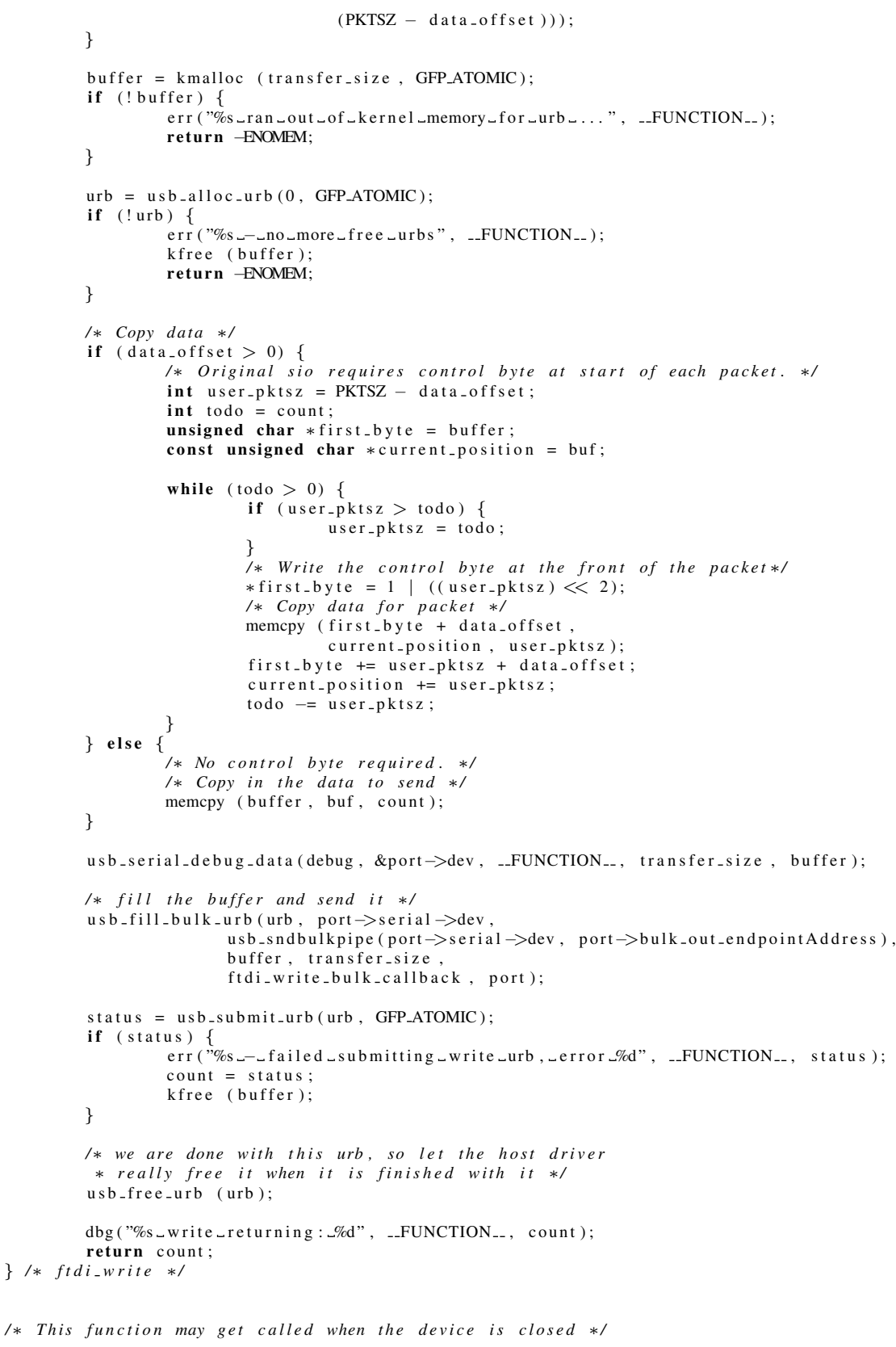




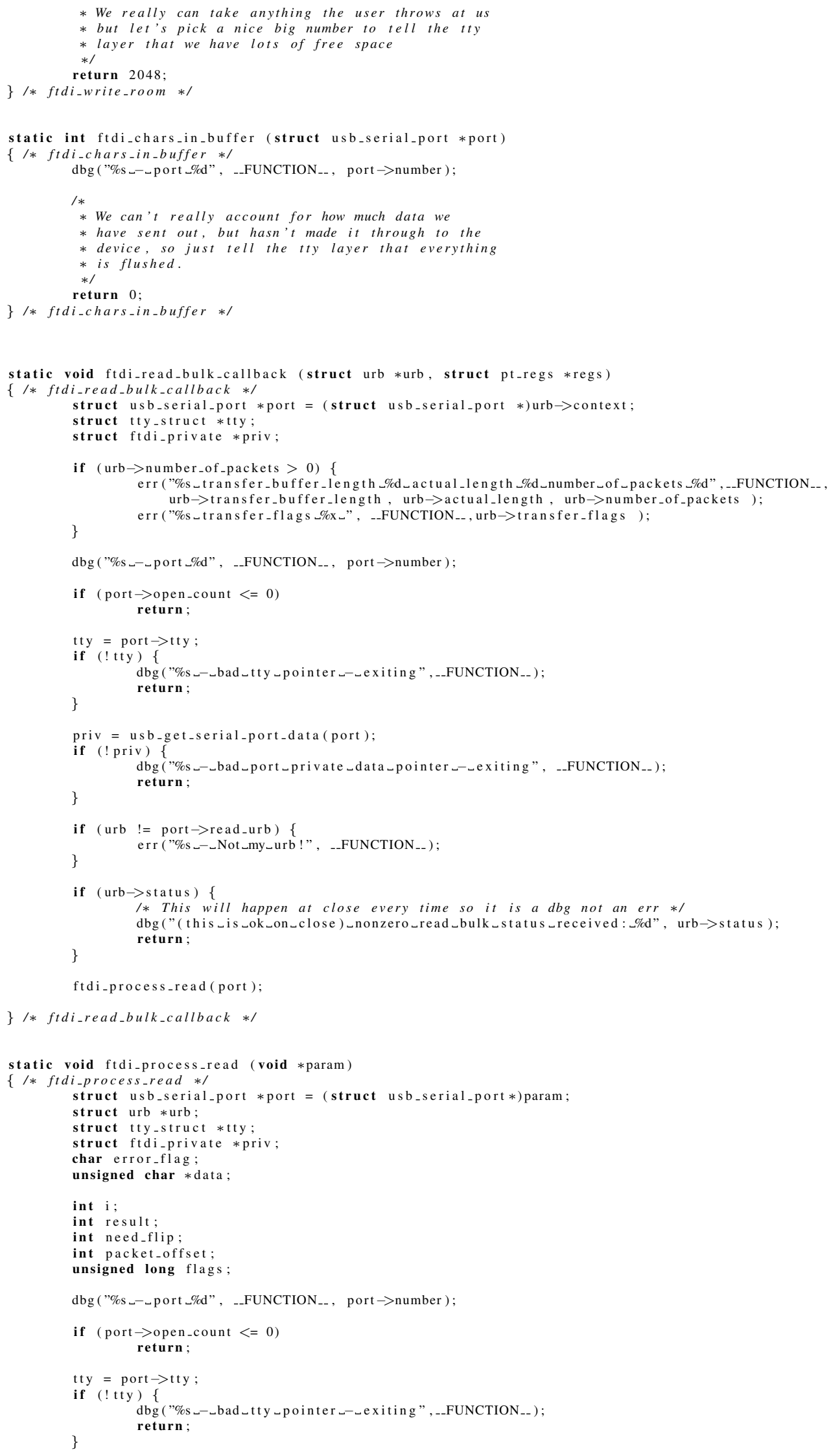




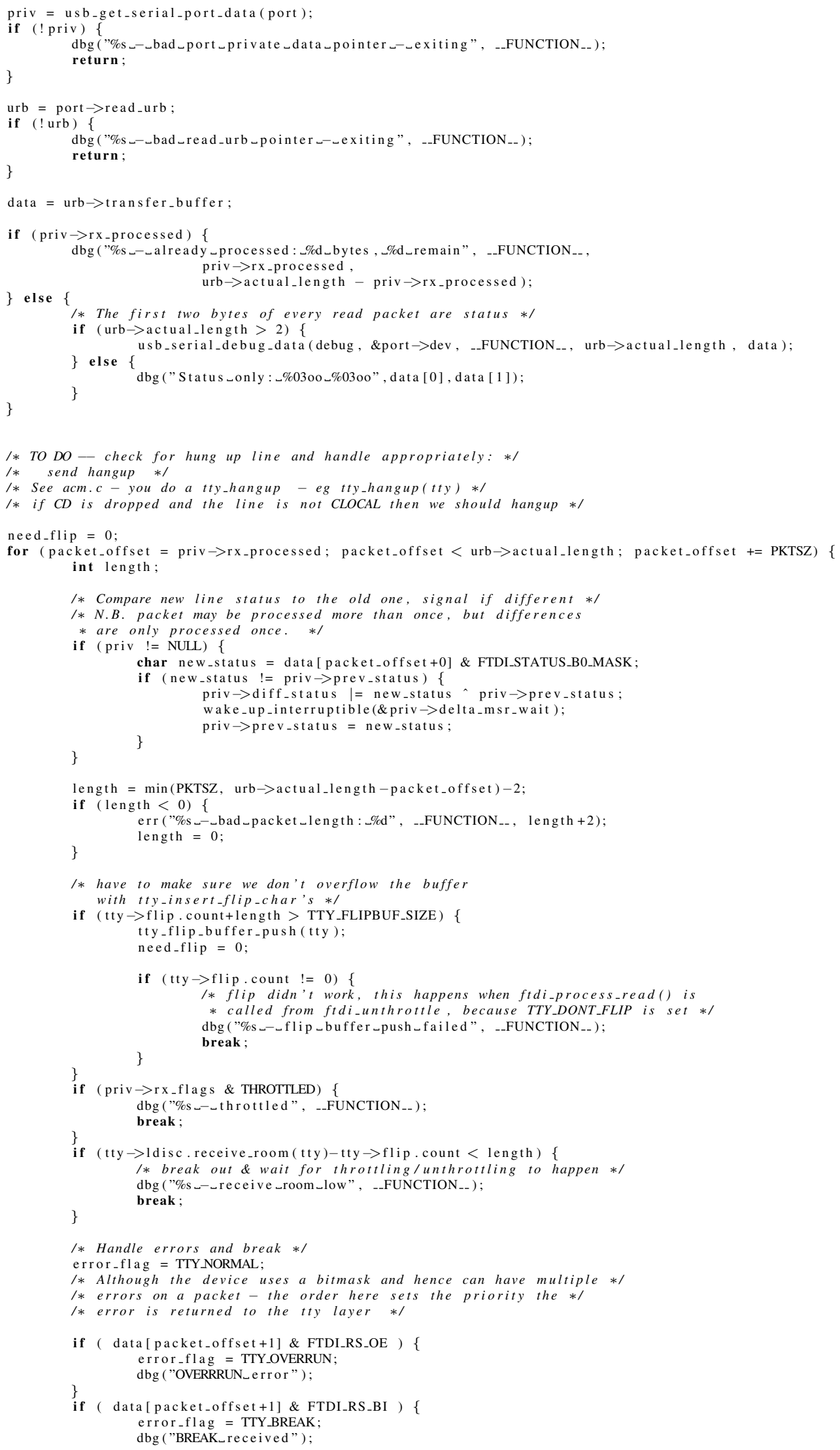


\}

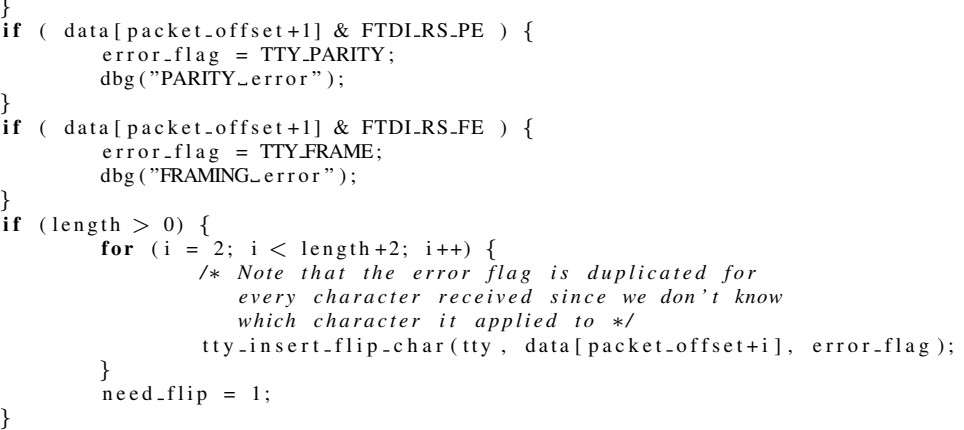

\# if d e f NOT_CORRECT_BUT_KEEPING_IT_FOR_NOW

I* if a parity error is detected you get status packets forever

until a character is sent without a parity error.

until a character is sent without a parity error.

ending stream of bad data - even though new data hasn' $t$ been sent.

Therefore I (bill) have taken this out.

However - this might make sense for framing errors and so on

so I am leaving the code in for now.

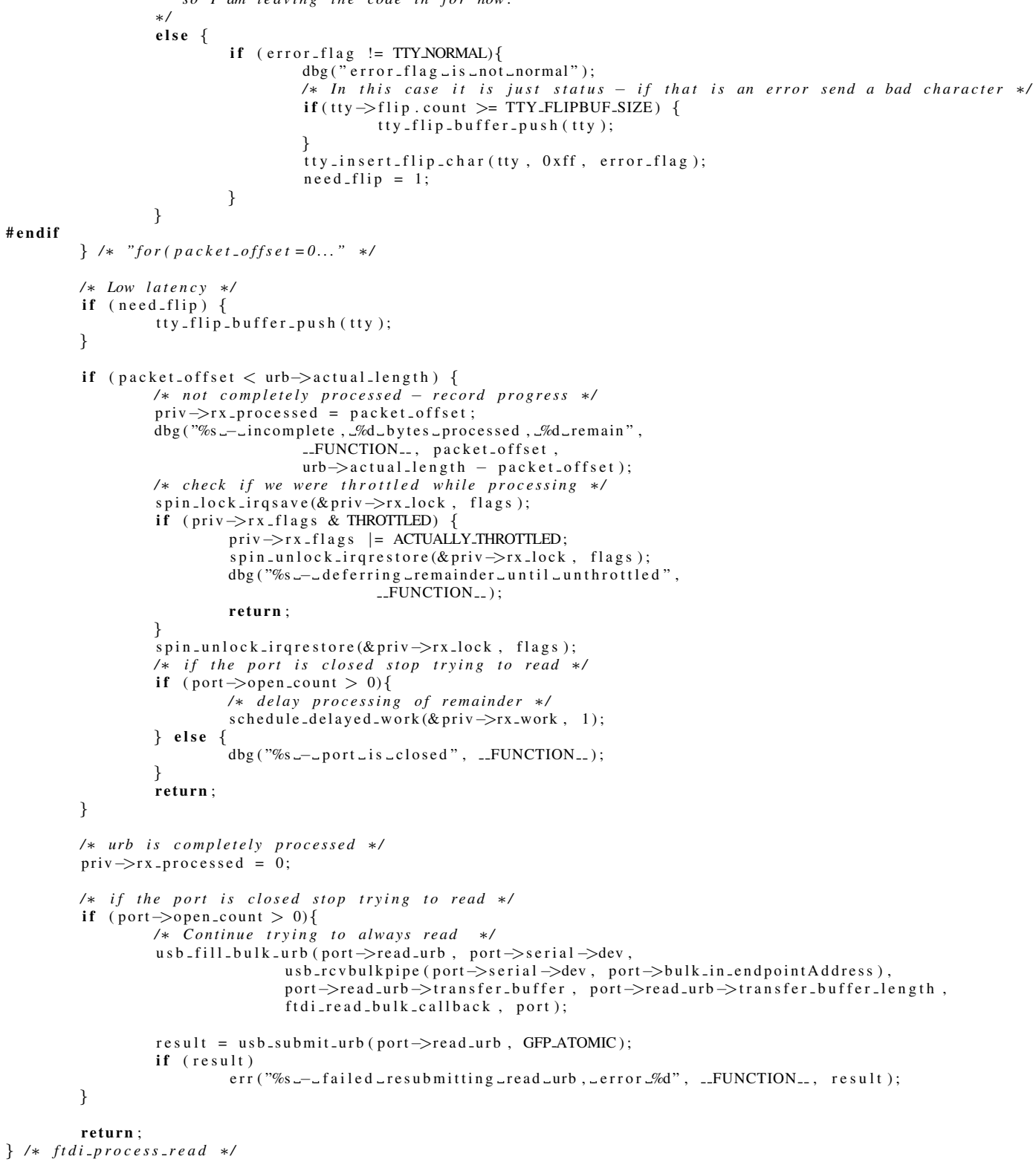

*/ 
static void ftdi_break_ctl( struct usb_serial_port *port, int break_state)

\{

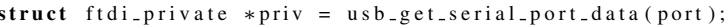

_-u 16 urb_value $=0$;

char buf [1];

/* break_state $=-1$ to turn on break, and 0 to turn off break */

/* see drivers/charltty io.c $_{\text {to }}$ see it used */

/* last_set_data_urb_value NEVER has the break bit set in it */

if (break_state) \{

\} else \{

urb_value $=$ priv $\rightarrow$ last_set_data_urb_value $\mid$ FTDI_SIO_SET_BREAK;

\}

urb_value $=$ priv $\rightarrow$ last_set_data_urb_value;

if (usb_control_msg (port $\rightarrow$ serial $\rightarrow$ dev, usb_sndctrlpipe (port $\rightarrow$ serial $\rightarrow$ dev, 0 ), FTDI_SIO_SET_DATA_REQUEST,

FTDI_SIO_SET_DATA_REQUEST_TYPE

urb_value, priv $\rightarrow$ interface

\} err ("\%s_FAILED_to_enable / disable_break_state_( state_was_\%d )", _.FUNCTION_-, break_state );

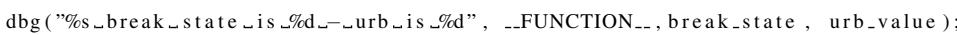

I* old_termios contains the original termios settings and tty $\rightarrow$ termios contains

* the new setting to be used

* WARNING: set_termios calls this with old_termios in kernel space

$* /$

static void ftdi_set_termios (struct usb_serial_port *port, struct termios *old_termios)

$\{/ *$ ftdi_termios $* /$

struct usb_device $*$ dev $=$ port $\rightarrow$ serial $\rightarrow$ dev;

unsigned int $\mathrm{cflag}=$ port $\rightarrow$ tty $\rightarrow$ termios $\rightarrow \mathrm{c}_{-} \mathrm{cflag}$;

struct ftdi-private $*$ priv = usb_get_serial_port_data(port);

-_ul6 urb_value; /* will hold the new flags $*$ I

char buf[1]; /* Perhaps I should dynamically alloc this? */

// Added for xon/xoff support

unsigned int iflag $=$ port $\rightarrow$ tty $\rightarrow$ termios $\rightarrow c_{-}$iflag;

unsigned char vstop;

unsigned char vstart

$\operatorname{dbg}(" \% s ", \quad$ _.FUNCTION__- $)$;

I* Force baud rate if this device requires it, unless it is set to BO. */

if (priv $\rightarrow$ force_baud \&\& ((port $\rightarrow$ tty $\rightarrow$ termios $\rightarrow c_{-}$cflag \& CBAUD) $!=$B0)) dbg ("\%s : $\_$forcing ubaud $\lrcorner$rate $\_$for $\lrcorner$this s device", __FUNCTION_- ); port $\rightarrow$ tty $\rightarrow$ termios $\rightarrow c_{-}$cflag $\&=$ CBAUD

\} port $\rightarrow$ tty $\rightarrow$ termios $\rightarrow c_{-}$cflag $\mid=$priv $\rightarrow$ force_baud

I* Force RTS-CTS if this device requires it. */

if ( priv $\rightarrow$ force_rtsets) \{ dbg("\%s:_forcing_rtscts_for_this_device”, _.FUNCTION__ );

\} port $\rightarrow$ tty $\rightarrow$ termios $\rightarrow c_{-}$cflag $\mid=$CRTSCTS

cflag $=$ port $\rightarrow$ tty $\rightarrow$ termios $\rightarrow c_{-}$cflag;

1* FIXME-For this cut I don't care if the line is really changing or not - so just do the change regardless - should be able to compare old_termios and tty $\rightarrow$ termios $* /$

I* NOTE These routines can get interrupted by

ftdi_sio_read_bulk_callback - need to examine what this

means - don't see any problems yet $* /$

I* Set number of data bits, parity, stop bits */

urb_value $=0$

urb_value $\mid=($ cflag \& CSTOPB ? FTDI_SIO_SET_DATA_STOP_BITS_2

urb_value $\mid=($ cflag \& PARENB

( $\mathrm{cflag} \&$ PARODD ? FTDI_SIO_SET_DATA_PARITY_ODD

FTDI_SIO_SET_DATA_PARITY_EVEN)

if ( $\mathrm{cflag} \&$ CSIZE) FTDI SIO SET DATA PARITY NONE);

witch (cflag \& CSIZE)

case CS5: urb_value I= 5; dbg("Setting_CS5"); break

case CS6: urb_value $\mid=6$; dbg ("Setting_CS6"); break

case CS7: urb_value $=7 ;$ dbg ("Setting_CS7"); break

case CS8: urb_value $=8$; dbg("Setting_CS8"); break;

default :

err ("CSIZE_was_set_but_not_CS5-CS8"); 


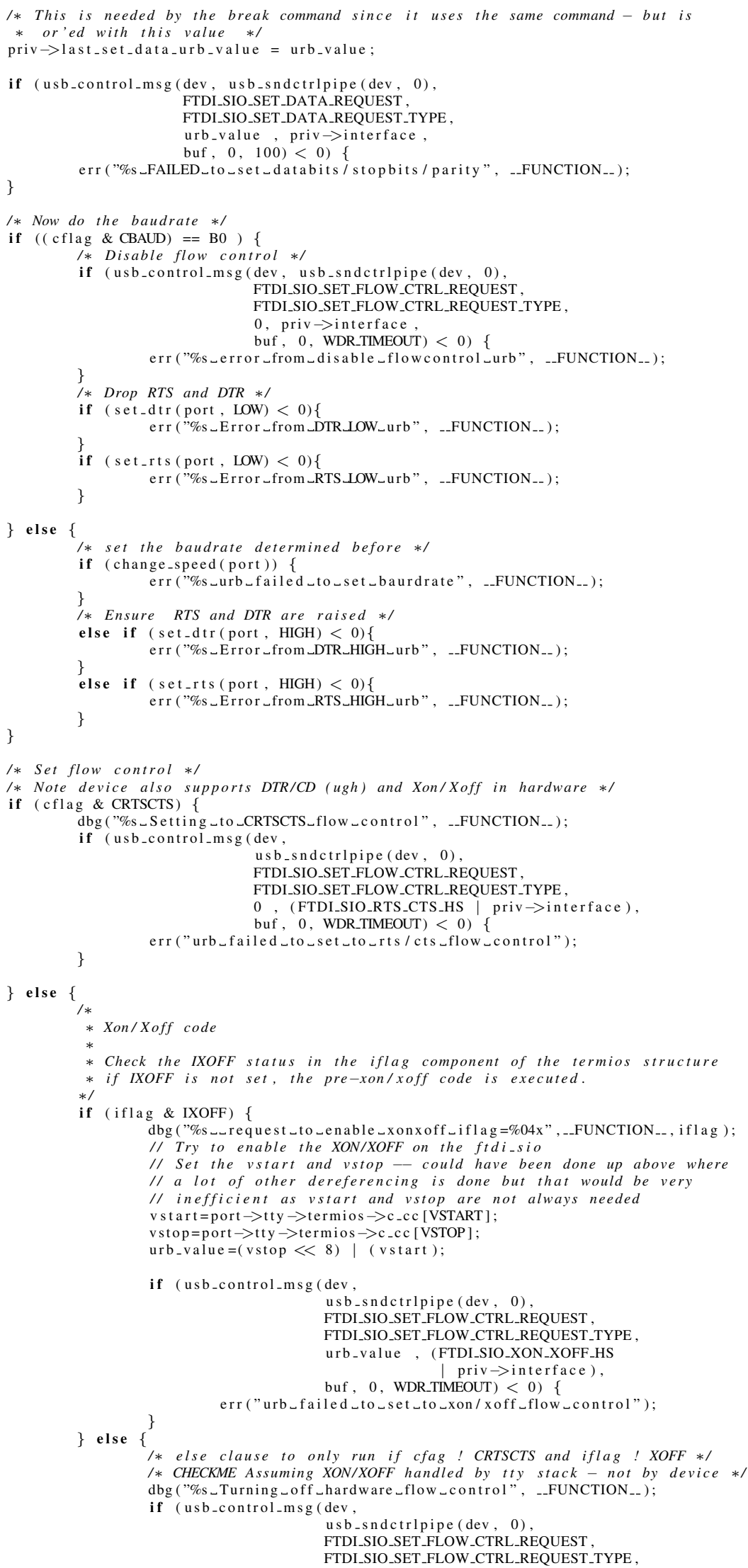


0 , priv $\rightarrow$ interface

buf, $\quad 0$, WDR_TIMEOUT) $<0$ ) \{

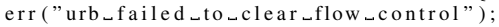

return

\} / * ftdi_termios */

static int ftdi_tiocmget (struct usb_serial_port *port, struct file *file)

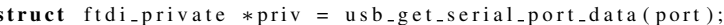
unsigned char buf $[2]$ :

int $\mathrm{ret}$;

dbg ("\%s _TIOCMGET", __FUNCTION_- );

switch (priv $\rightarrow$ chip_type)

case $\mathrm{SIO}$ :

/* Request the status from the device */

if $($ ( ret $=$ usb_control_msg $($ port $\rightarrow$ serial $\rightarrow$ dev

usb_rcvetrlpipe (port $\rightarrow$ serial $\rightarrow$ dev, 0$)$,

FTDI_SIO_GET_MODEM_STATUS_REQUEST,

FTDI_SIO_GET_MODEM_STATUS_REQUEST_TYPE,

0,0 ,

buf , 1 , WDR_TIMEOUT) $)<0$ ) \{

err ("\%s_Could_not_get_modem_status_of_device___err :_\%d", __FUNCTION_-

$$
\text { ret ); }
$$

reak ;

return (ret);

case FT8U232AM:

case FT232BM

case FT2232C

1* the 8U232AM returns a two byte value (the sio is a l byte value) - in the same format as the data returned from the in point *l

if $(($ ret $=$ usb_control_msg $($ port $\rightarrow$ serial $\rightarrow$ dev

usb_rcvetrlpipe ( port $\rightarrow$ serial $\rightarrow$ dev, 0$)$,

FTDI_SIO_GET_MODEM_STATUS_REQUEST,

FTDI_SIO_GET_MODEM_STATUS_REQUEST_TYPE,

0 , priv $\rightarrow$ interface

buf, 2 , WDR_TIMEOUT) $)<0$ ) \{ err ("\%s_Could_not_get_modem_status_of_device__-_err : _\%d", _.FUNCTION_ret);

return $(r e t)$

break

default :

return -EFAULT

return (buf [0] \& FTDI_SIO_DSR_MASK ? TIOCM_DSR : 0)

(buf [0] \& FTDI_SIO_CTS_MASK ? TIOCM_CTS : 0)

(buf [0] \& FTDI_SIO_RI_MASK ? TIOCM_RI : 0 )

(buf [0] \& FTDI_SIO_RLSD_MASK ? TIOCM_CD : 0 ) priv $\rightarrow$ last $\mathrm{dtr}_{-} \mathrm{rts}$

static int ftdi_tiocmset(struct usb_serial_port *port, struct file $*$ file, unsigned int set, unsigned int clear)

int ret

dbg ("\%s_TIOCMSET_set:\%02X_clear:\%02X",_FUNCTION_, set, clear);

if ( set \& TIOCM_DTR) \{

dbg ("\% _ setting _TIOCM_DTR", _._FUNCTION_-- );

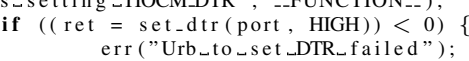
return ( ret);

if (set \& TIOCM RTS ) \{

dbg ("\% _ setting _TIOCM_RTS",__FUNCTION_- $)$;

if $\left(\left(\right.\right.$ ret $=$ set_rts $\left(\right.$ port,$\left.\left.\left.H_{G H}\right)\right)<0\right)\{$ err("Urb_to_set_RTS_failed");

\} return (ret)

if ( clear \& TIOCM_DTR)

dbg ("\%s_clearing $\_$TIOCM_DTR", __FUNCTION__ );

if $(($ ret $=$ set $-d t r($ port, LOW $))<0)\{$, err("Urb_to_unset_DTR_failed");

\} return (ret)

if

(clear \& TIOCM_RTS) \{

dbg ("\%s_clearing _TIOCM_RTS", _._FUNCTION_- );

if $(($ ret $=$ set_rts $($ port, LOW $))<0)\{$

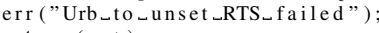

return (ret); 


\section{\} $\}$ \\ return $(0)$;}

static int ftdi_ioctl (struct usb_serial_port *port, struct file * file, unsigned int cmd, unsigned long arg)

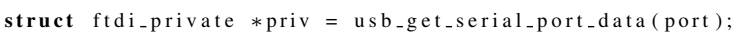

in t ret, mask:

$\operatorname{dbg}\left(" \% s \_c m d \_0 x \% 04 x ", \quad\right.$ _-FUNCTION_-, cmd );

(* Based on code from acm.c and others */

switch (cmd) \{

case TIOCMBIS: /* turns on (Sets) the lines as specified by the mask */

$\operatorname{dbg}(" \%$ s_TIOCMBIS", _-FUNCTION_- $)$

if (get_user(mask, (unsigned long _. user *) arg))

return -EFAULT;

if (mask \& TIOCM_DTR) \{

if $(($ ret $=$ set_dtr $($ port, $\mathrm{HIGH}))<0)\{$ err ("Urb_to_set _DTR_failed");

\} return( ret);

if

\& TIOCM_RTS ) \{

if $(($ ret $=$ set $r$ ts $($ port, HIGH $))<0)$

err ("Urb_to_set_RTS_failed")

\}

\}

return (ret)

return $(0)$

break ;

case TIOCMBIC: /* turns off (Clears) the lines as specified by the mask */ dbg ("\%s_TIOCMBIC", _FUNCTION_- );

if (get_user (mask, (unsigned long _user *) arg))

return-EFAULT;

if (mask \& TIOCM_DTR) \{

if $\left(\left(\right.\right.$ ret $=$ set $_{-} \mathrm{dtr}($ port, LOW $\left.\left.)\right)<0\right)\{$ err ("Urb_to_unset_DTR_failed")

\} return (ret);

\}

(mask \& TIOCM_RTS ) \{

if $(($ ret $=$ set_rts $($ port, LOW $))<0)\{$ err ("Urb to tounset $\_$RTS failed")

\} return( ret);

return $(0)$

break;

$/ *$

* I had originally implemented TCSET $\{A, S\}\{, F, W\}$ and

* TCGET $\{A, S\}$ here separately, however when testing $I$

* found that the higher layers actually do the termios

* conversions themselves and pass the call onto

* ftdi_sio_set_termios.

$* /$

case TIOCGSERIAL: $/ *$ gets serial port data */ return get_serial_info(port, (struct serial_struct__user *) arg);

case TIOCSSERIAL: $/ *$ sets serial port data $* /$ return set_serial_info(port, (struct serial_struct__user *) arg)

* Wait for any of the 4 modem inputs (DCD, RI,DSR,CTS) to change

* - mask passed in arg for lines of interest

* (use |'ed TIOCM_RNG/DSR/CD/CTS for masking.

* Caller should use TIOCGICOUNT to see which one it was.

* This code is borrowed from linux/drivers/char/serial.c case TIOCMIWAIT:

while (priv != NULL)

interruptible_sleep_on(\&priv $\rightarrow$ delta_msr_wait)

/* see if a signal did it */

if ( signal_pending (current))

else \{ return-ERESTARTSYS;

char $\operatorname{diff}=$ priv $\rightarrow$ diff $_{-}$status $_{\text {; }}$

if $(\operatorname{diff}==0)\{$

return -EIO; $/ *$ no change $\Rightarrow$ error */

/* Consume all events */ 


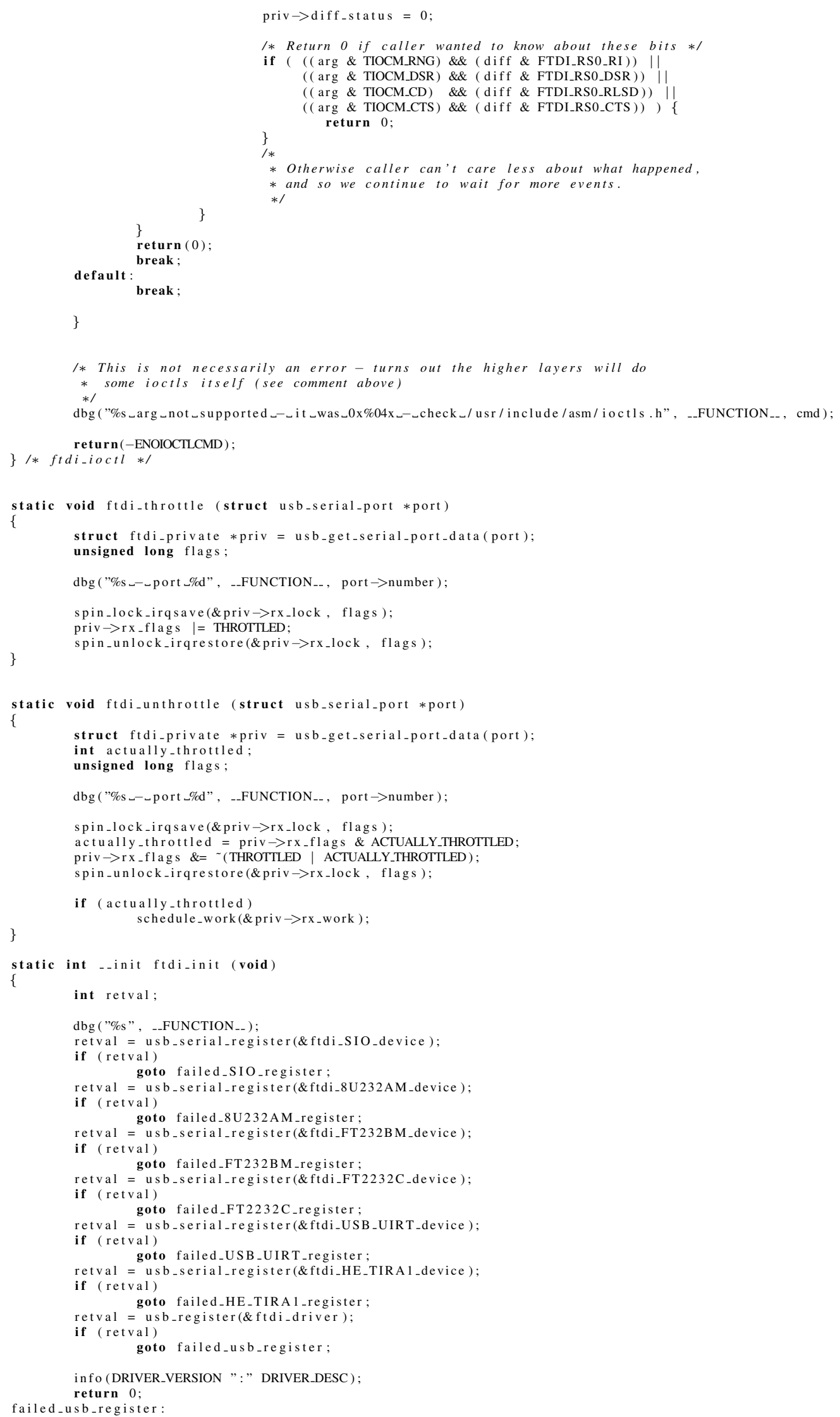




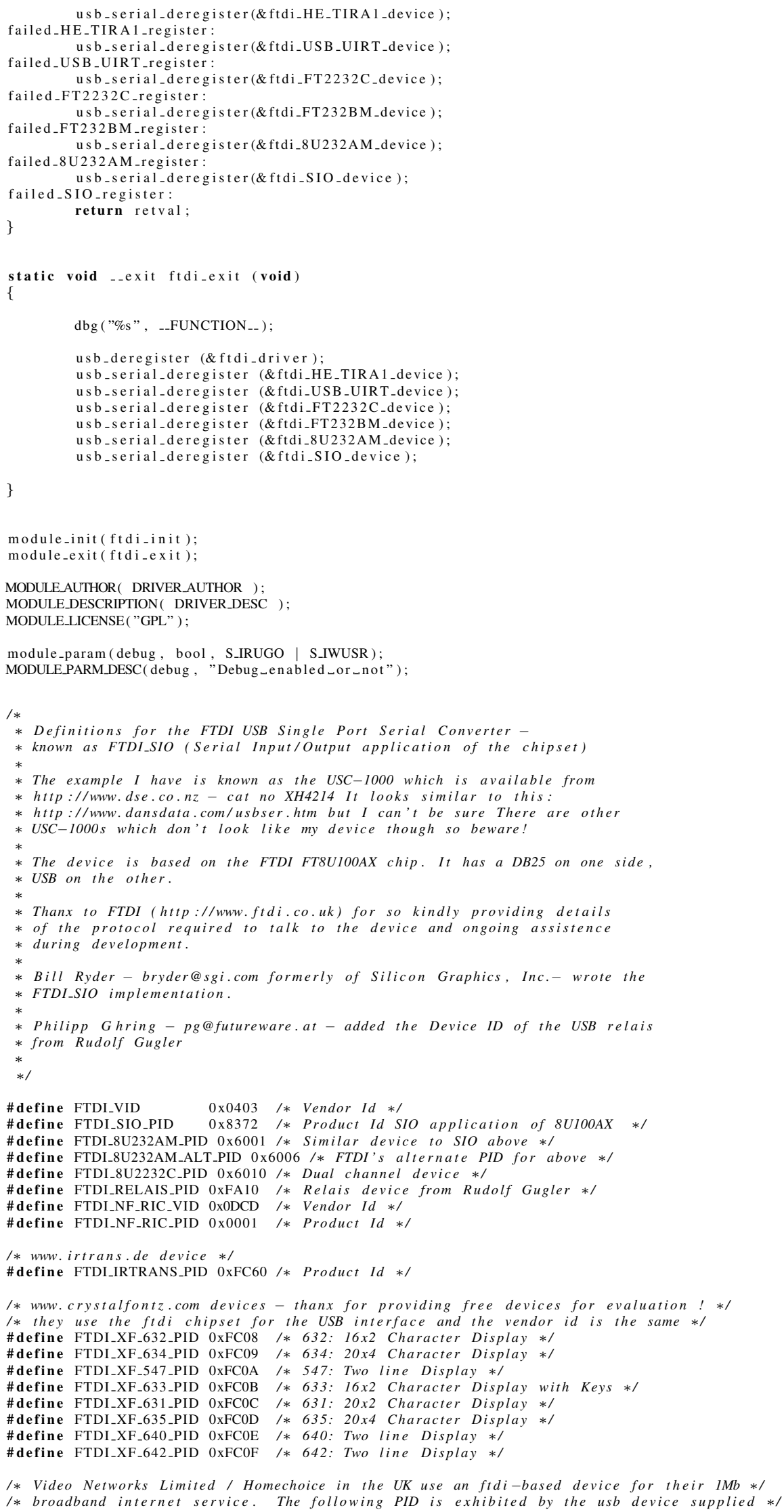




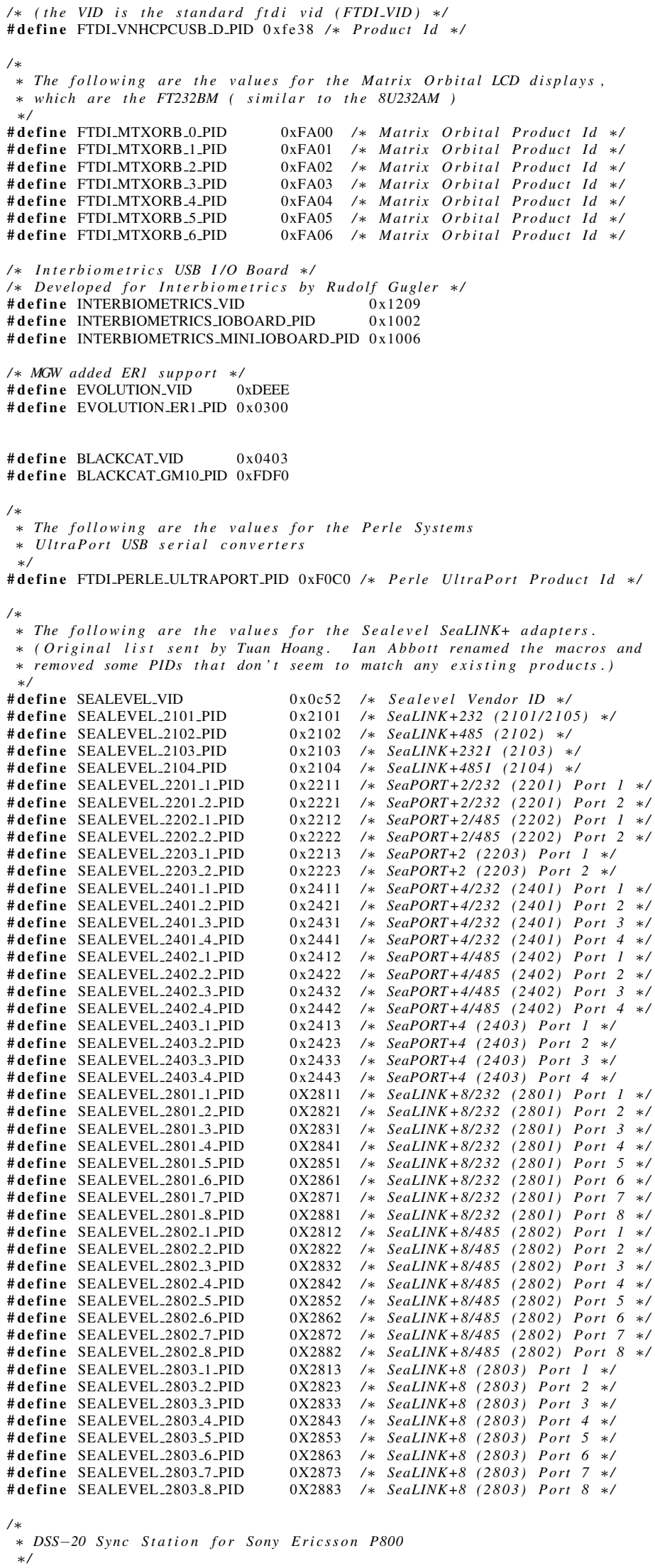




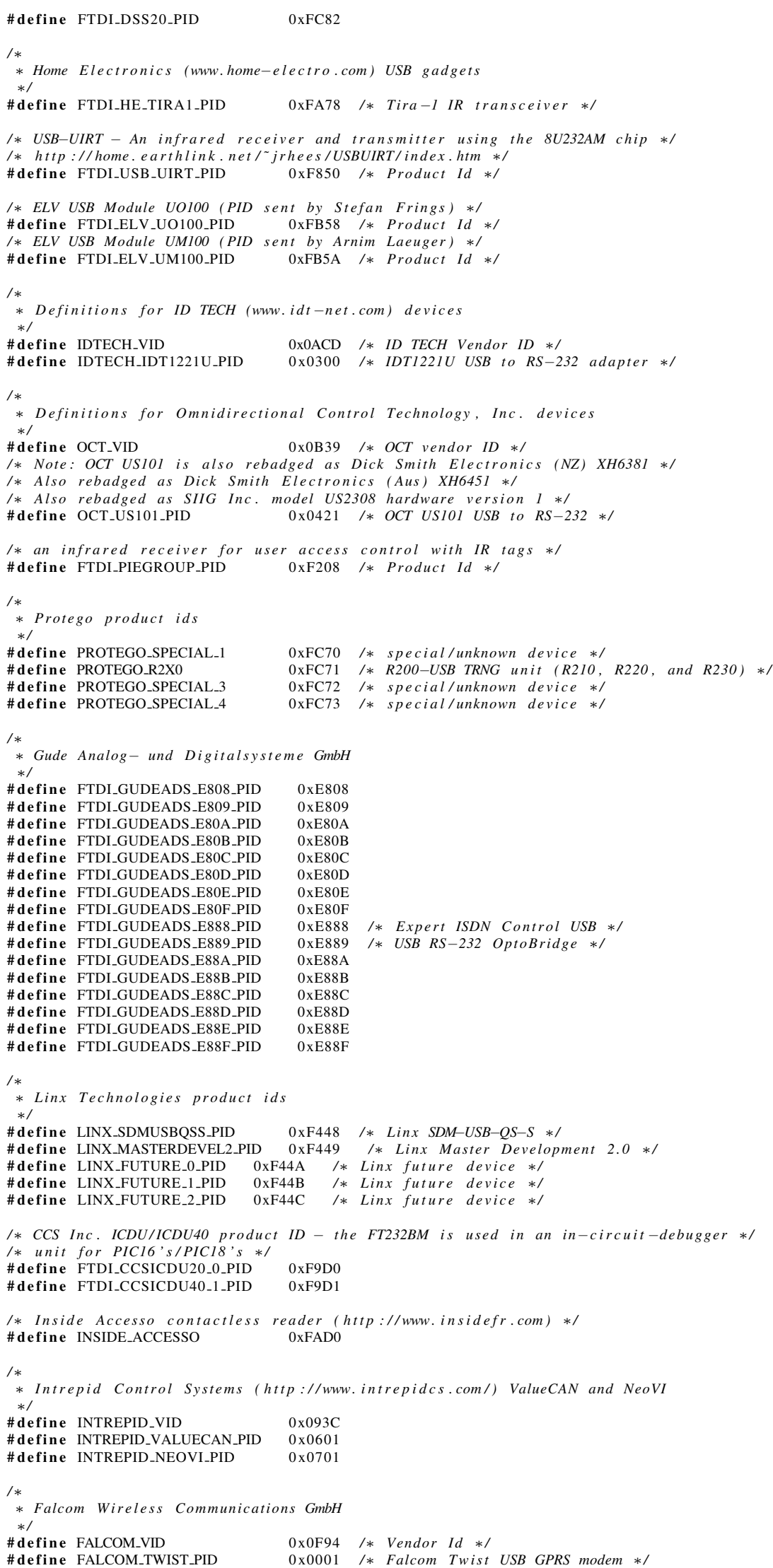




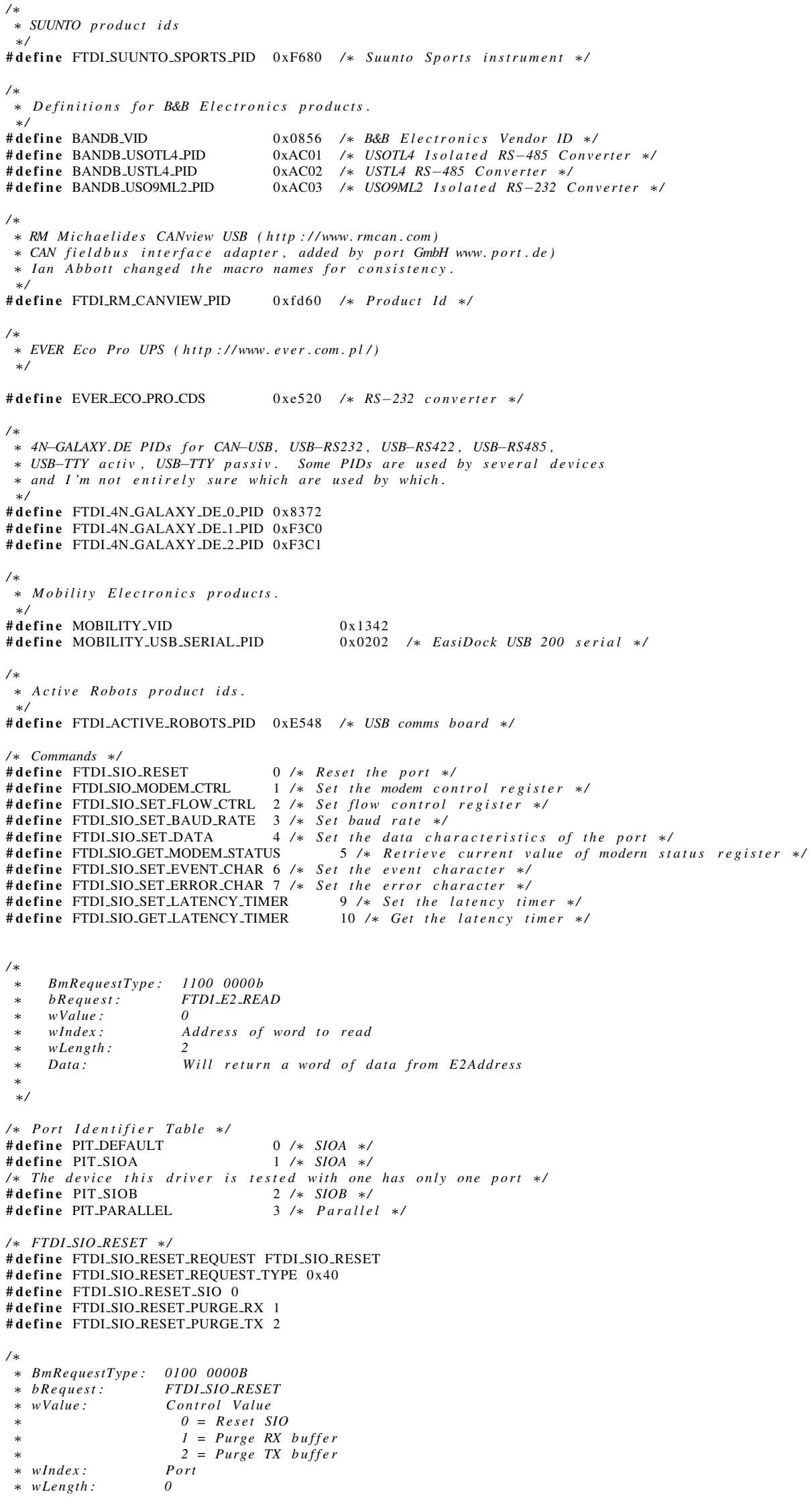




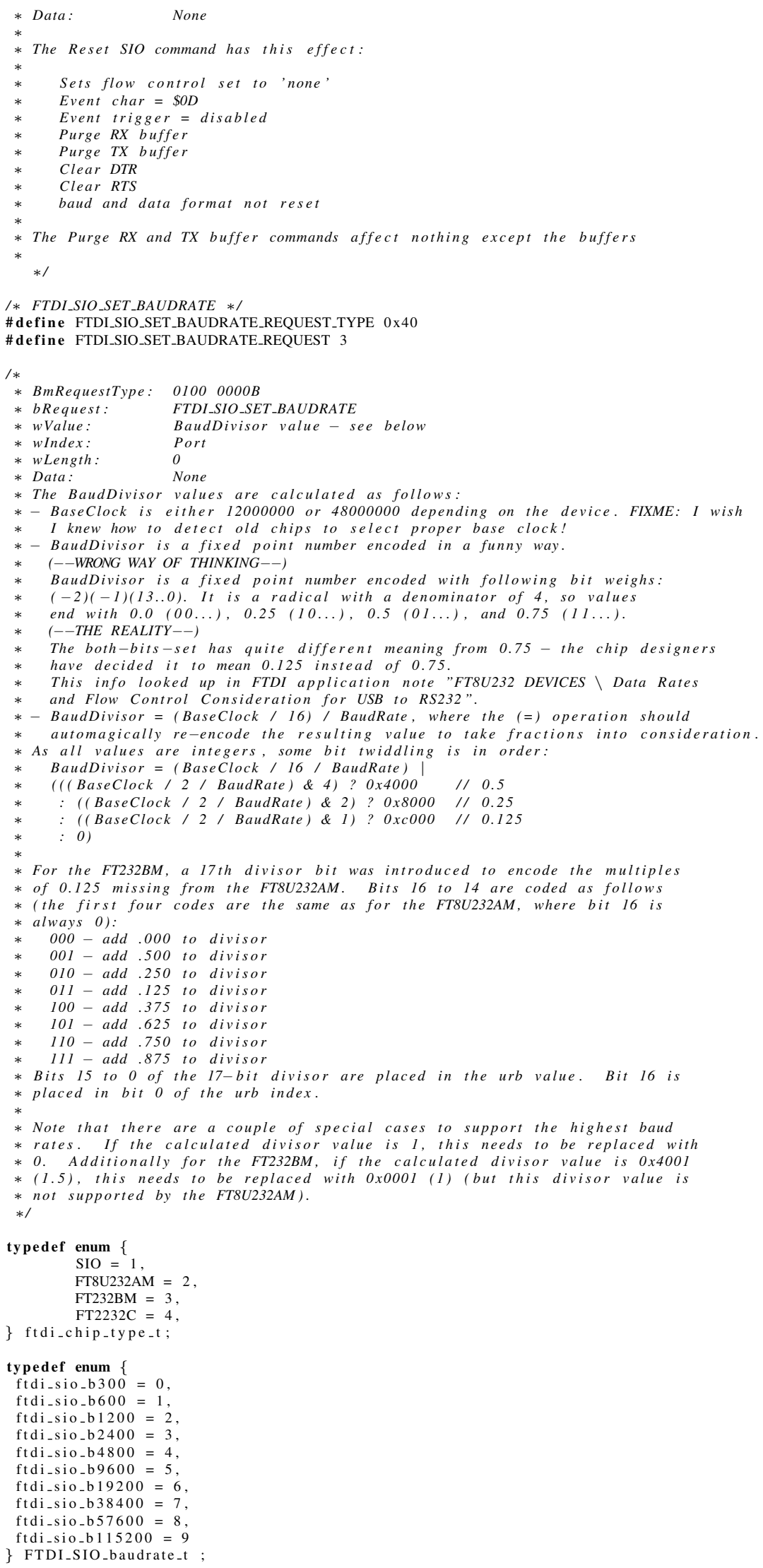




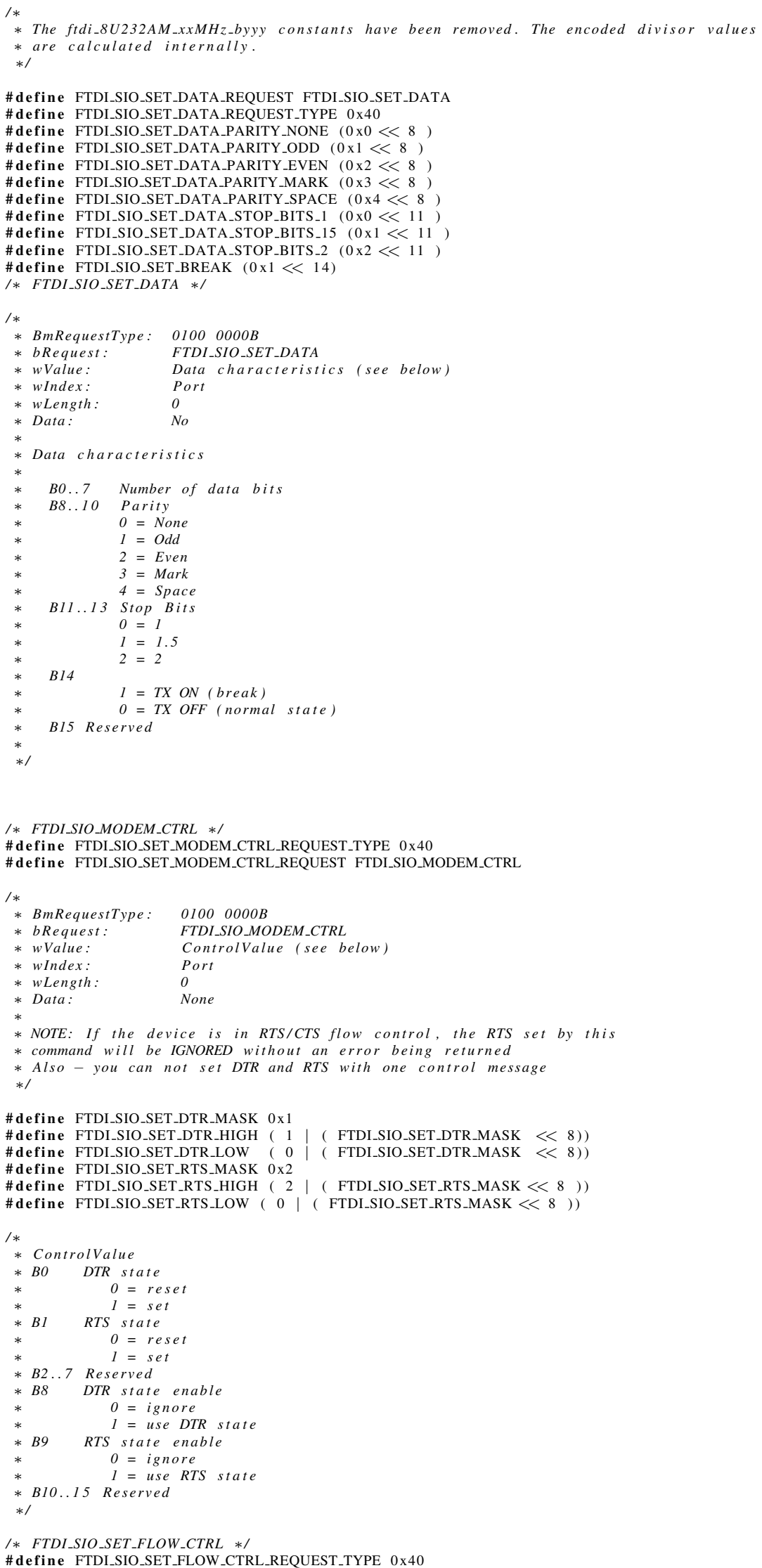


\#define FTDI_SIO_SET_FLOW_CTRL_REQUEST FTDI_SIO_SET_FLOW_CTRL

\#define FTDI_SIO_DISABLE_FLOW_CTRL $0 \times 0$

\#define FTDI_SIO_RTS_CTS_HS $(0 \times 1<<8)$

\#define FTDI_SIO_DTR_DSR_HS $(0 \times 2<<8)$

\#define FTDI_SIO_XON_XOFF_HS $(0 \times 4<<8)$

/*

* BmRequestType: $01000000 b$

bRequest: FTDI_SIOSET_FLOW_CTRL

wValue: $\quad$ Xoff/Xon

wIndex: Protocol/Port - hIndex is protocl / lindex is port

whength: $\quad 0$

hIndex protocol is:

BO Output handshaking using RTS/CTS

$0=$ disable

$1=$ enabled

BI Output handshaking using DTR/DSR

$0=$ disabled

$I$ = enabled

B2 Xon/Xoff handshaking

$0=$ disabled

$I$ = enabled

* A value of zero in the hIndex field disables handshaking

* If Xon/Xoff handshaking is specified, the hValue field should contain the XOFF character

* and the lValue field contains the XON character.

* Set the timeout interval. The FTDI collects data from the slave

* device, transmitting it to the host when either A) 62 bytes are

* received, or $B)$ the timeout interval has elapsed and the buffer

* contains at least 1 byte. Setting this value to a small number

* can dramatically improve performance for applications which send

* small packets, since the default value is $16 \mathrm{~ms}$.

\#define FTDI_SIO_GET_LATENCY_TIMER_REQUEST FTDI_SIO_GET_LATENCY_TIMER

\#define FTDI_SIO_GET_LATENCY_TIMER_REQUEST_TYPE 0xC0

* BmRequestType: 1100 0000b

FTDI_SIO_GET LATENCY TIMER

* wValue: $\quad 0$

* wIndex: Port

wLength: $\quad 0$

Data: latency (on return)

*/

/*

* FTDI_SIO_SET_LATENCY_TIMER

* Set the timeout interval. The FTDI collects data from the slave

* device, transmitting it to the host when either A) 62 bytes are

* received, or $B$ ) the timeout interval has elapsed and the buffer

* contains at least 1 byte. Setting this value to a small number

* can dramatically improve performance for applications which send

* small packets, since the default value is $16 \mathrm{~ms}$.

\#define FTDI_SIO_SET_LATENCY_TIMER_REQUEST FTDI_SIO_SET_LATENCY_TIMER

\#define FTDI_SIO_SET_LATENCY_TIMER_REQUEST_TYPE $0 \times 40$

* BmRequestType: 0100 0000b

* bRequest: FTDI_SIO_SET_LATENCY_TIMER

* wValue: $\quad$ Latency (milliseconds)

* wIndex: Port

* wLength: $\quad 0$

* Data: None

* walue:

* B0..7 Latency timer

$*$

* FTDI_SIO_SET_EVENT_CHAR

* Set the special event character for the specified communications port.

* If the device sees this character it will immediately return the

* data read so far - rather than wait $40 \mathrm{~ms}$ or until 62 bytes are read

* which is what normally happens.

$* /$

\#define FTDI_SIO_SET_EVENT_CHAR_REQUEST FTDI_SIO_SET_EVENT_CHAR

\#define FTDI_SIO_SET_EVENT_CHAR_REQUEST_TYPE $0 \times 40$ 


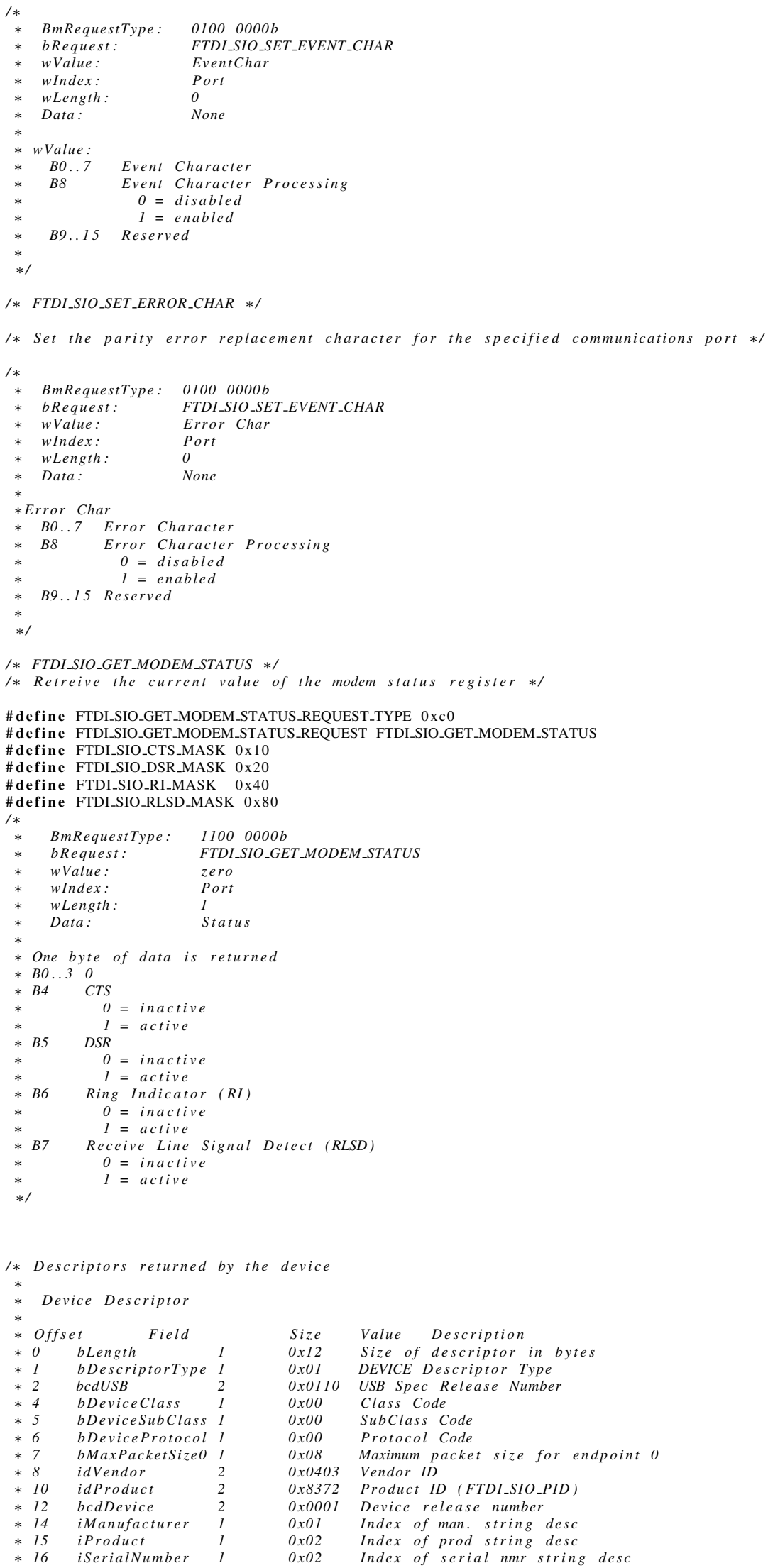




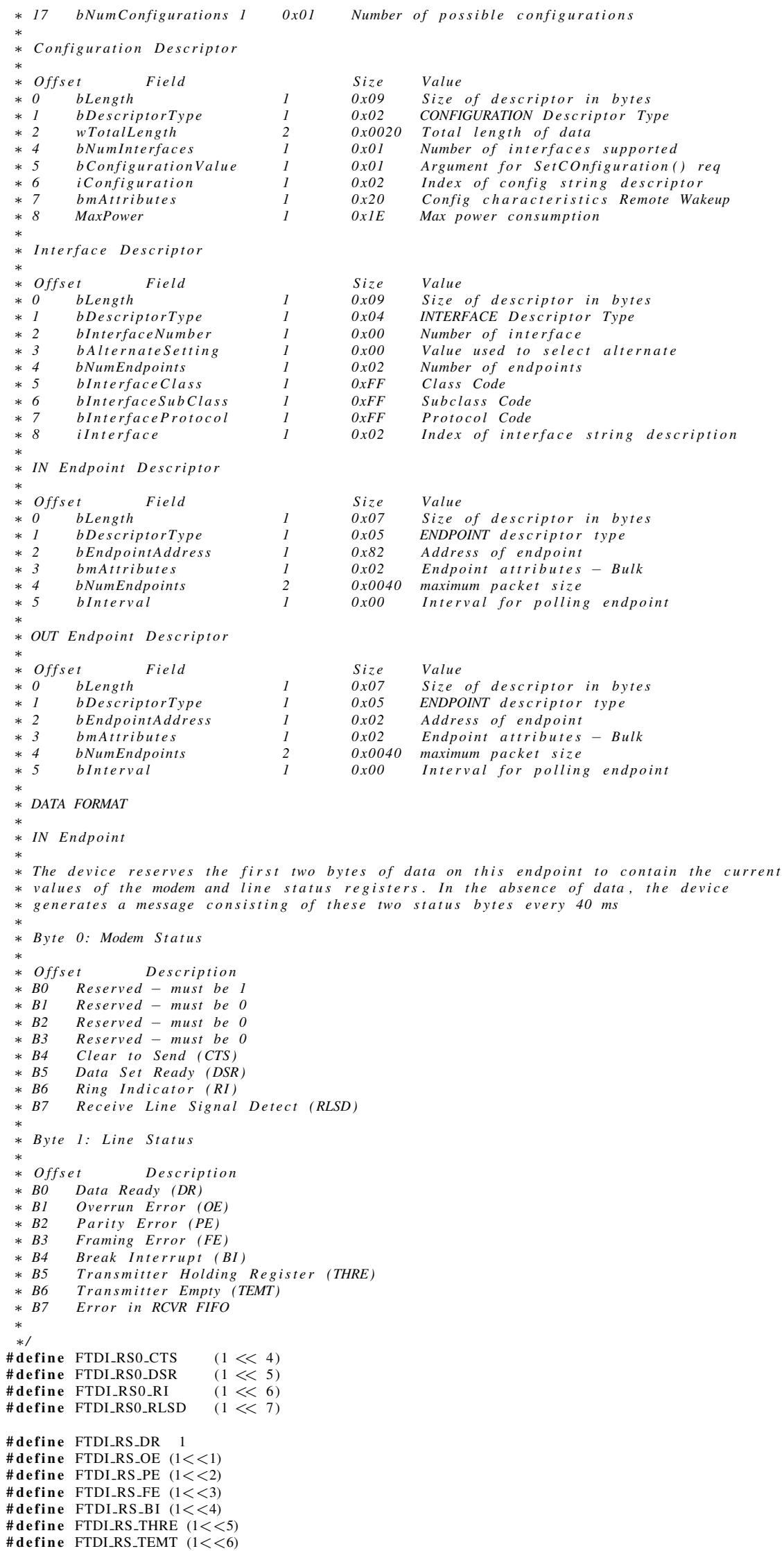


\#define FTDI_RS_FIFO $(1<<7)$

/*

* OUT Endpoint

* This device reserves the first bytes of data on this endpoint contain the length

* and port identifier of the message. For the FTDI USB Serial converter the port

* identifier is always 1 .

* Byte 0: Line Status

* Offset Description

* BO Reserved - must be 1

* BI Reserved - must be 0

* B2..7 Reserved - must be 0

*/ 


\section{REFERENCES}

[1] Lars Cremean, William Dunbar, Dave Van Gogh, Jason Hickey, Eric Klavins, Jason Meltzer and Richard Murray, "The Caltech Multi-Vehicle Wireless Testbed", in Proceedings of the 41 st IEEE Conference on Decision and Control, Las Vegas, NA, 2002, pp. TuA03-4.

[2] Raffaello D'Andrea and Michael Babish, "The RoboFlag Testbed”, in Proceedings of the American Control Conference, Denver, CO, 2003, pp. 656-660.

[3] Ellis King, Yoshi Kuwata, Mehdi Alighanbari, Luca Bertuccelli, and Johnathan How, "Coordination and Control Experiments on a Multi-vehicle Testbed", in Proceeding of the 2004 American Control Conference, Boston, MA, 2004, pp. 5315-5320.

[4] Rafael Fierro and EzzAldeen Edwan, "The OSU Multi-vehicle Coordination Testbed", in 45th Midwest Symposium on Circuits and Systems, vol. 3, 2002, pp. III-41-III-44.

[5] Gabe Hoffmann, Dev Gorur Rajnarayan, Steven Waslander, David Dostal, Jung Soon Jang, and Claire Tomlin, "The Stanford Testbed of Autonomous Rotorcraft for Multi Agent Control (STARMAC)", in 23rd Digital Avionics Systems Conference, vol. 2, 2004, pp. 12.E.4-1.

[6] Mario Valenti, Brett Bethke, Gaston Fiore, Jonathan How, and Eric Feron, "Indoor Multi-Vehicle Flight Testbed for Fault Detection, Isolation, and Recovery", in AIAA Guidance, Navigation, and Control Conference, Keystone, CO, 2006, pp. 6200-6218.

[7] Vladimeros Vladimerou, Andrew Stubbs, Joel Rubel, Adam Fulford, Jeffrey Strick, Geir Dullerud, "A Hovercraft Testbed for Decentralized and Cooperative Control”, in Proceeding of the 2004 American Control Conference, Boston, MA, 2004, pp. 12361241

[8] Adrian Ilie and Greg Welch, "Ensuring Color Consistency Across Multiple Cameras", in Proceedings of the Tenth IEEE International Conference on Computer Vision, 2005.

[9] James Bruce, Tucker Balch, and Manuela Veloso, "Fast and Cheap Color Image Segmentation for Interactive Robots", School of Computer Science, Carnegie Mellon University, Pittsburgh, PA, 2000.

[10] Richard Duda, Peter Hart, David Stork. 2001. Pattern Classification. John Wiley \& Sons. 
[11] Rafael Gonzalez, Richard Woods, Steven Eddins. 2004. Digital Image Processing. Pearson Prentice Hall.

[12] 68-95-99.7 Rule. Internet Source, 1 November 2006, available from http://en.wikipedia.org/wiki/Standard_deviation; accessed 1 November 2006.

[13] CCTV Factory. Internet Source, 3 May 2006, available from http://www.cctvfactory.com; accessed 3 May 2006.

[14] Linux Media Labs. Internet Source, 3 May 2006, available from http://www.linuxmedialabs.com; accessed 3 May 2006.

[15] Evolution Robotics. Internet Source, 15 January 2006, available from http://www.evolution.com/er1; accessed 15 January 2006.

[16] The Player/Stage Project. Internet Source, 2 November 2005, available http://playerstage.sourceforge.net/; accessed 2 November 2005.

[17] Roland Siegwart, Illah R. Nourbakhsh. 2004. Introduction to Autonomous Mobile Robots. The MIT Press.

[18] Sordalen, O.J. and DeWit, C. Canudas. 1992. "Exponential Control Law for a Mobile Robot: Extension to Path Following". Proceedings of the International Control Conference on Robotics and Automation, Nice, France, pp. 2158-2163.

[19] DeSantis, R.M.. 1995. "Path-Tracking for Car-Like Robots with Single and Double Steering". IEEE Transactions on Vehicular Technology. 44:366-377.

[20] L. Ljung, System Identification: Theory for the User, Prentice Hall, 1987.

[21] R. S. Sanchez Pena and M. Sznaier, Robust Systems Theory and Applications, John Wiley, New Jersey, 1998.

[22] T. Inanc, M. Sznaier, P. A. Parrilo and R. S. Sanchez Pena, "Robust Identification with Mixed Parametric/Nonparametric Models and Time/Frequency-Domain Experiments: Theory and an Application", IEEE Transactions on Control Systems Technology, July 2001, Vol. 9, No. 4, pp 608-617. 


\section{VITA}

Travis Alan Riggs was born in Louisville, KY. Raised in neighboring Oldham County, Travis attended the county schools, graduating third in his class at Oldham County Senior High. With the option of attending the United States Naval Academy and Georgia Institute of Technology, Travis chose the University of Louisville as his home for undergraduate studies in Electrical Engineering. He completed his B.S. in 2005 and hopes to complete his M.Eng. in 2006. He now resides in Louisville with his lovely wife, Jaime. 